\title{
Minimum-Distortion Embedding
}

\author{
Akshay Agrawal \\ Alnur Ali \\ Stephen Boyd \\ akshayka@cs.stanford.edu \\ alnurali@stanford.edu \\ boyd@stanford.edu
}

August 26, 2021 


\section{Contents}

1 Introduction 1

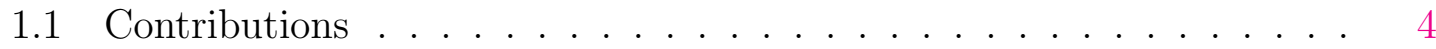

1.2 Outline . . . . . . . . . . . . . . . . . . . 4

1.3 Related work . . . . . . . . . . . . . . 5

I Minimum-Distortion Embedding 9

2 Minimum-Distortion Embedding 10

2.1 Embedding . . . . . . . . . . . . . . . . . . . . . . . . . . . 10

2.2 Distortion . . . . . . . . . . . . . . . . . . . . . . 11

2.3 Minimum-distortion embedding . . . . . . . . . . . . . . . 15

2.4 Constraints . . . . . . . . . . . . . . . . . . . . . . . 19

2.5 Simple examples . . . . . . . . . . . . . . . . . . . . . . . . . . 24

2.6 Validation . . . . . . . . . . . . . . . . 26

3 Quadratic MDE Problems 30

3.1 Solution by eigenvector decomposition . . . . . . . . . . . . 31

3.2 Historical examples . . . . . . . . . . . . . . . . . . . . . 33

4 Distortion Functions 37

4.1 Functions involving weights . . . . . . . . . . . 37

4.2 Functions involving original distances . . . . . . . . . . . . . . 42

4.3 Preprocessing . . . . . . . . . . . . . . . . 46

II Algorithms 51

5 Stationarity Conditions $\quad 52$ 
5.1 Centered MDE problems . . . . . . . . . . . . . . . 54

5.2 Anchored MDE problems . . . . . . . . . . . . . . . . 55

5.3 Standardized MDE problems . . . . . . . . . . . . . 56

6 Algorithms 59

6.1 A projected quasi-Newton algorithm . . . . . . . . . . . 60

6.2 A stochastic proximal algorithm . . . . . . . . . . . . . 65

7 Numerical Examples $\quad 68$

7.1 Quadratic MDE problems .................. 69

7.2 Other MDE problems . . . . . . . . . . . . . . . . . 72

7.3 A very large problem . . . . . . . . . . . . . . . . . 75

7.4 Implementation . . . . . . . . . . . . . . . . 79

III Examples 84

8 Images 85

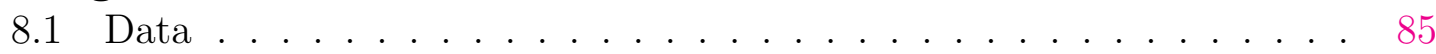

8.2 Preprocessing . . . . . . . . . . . . . . . . . . . 85

8.3 Embedding . . . . . . . . . . . . . . . . . . 86

9 Networks $\quad 96$

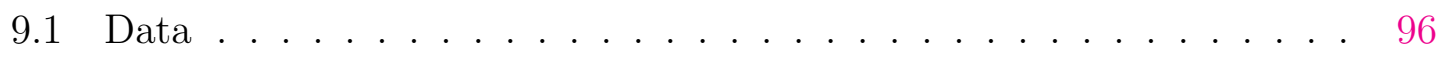

9.2 Preprocessing . . . . . . . . . . . . . . . . . . 100

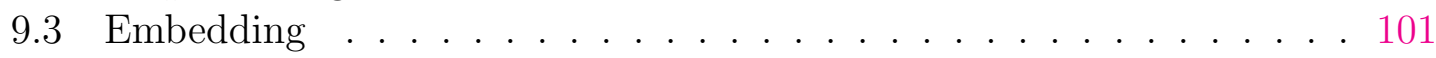

10 Counties $\quad 109$

10.1 Data . . . . . . . . . . . . . . . . . . 109

10.2 Preprocessing . . . . . . . . . . . . . . . . . . . . 109

10.3 Embedding . . . . . . . . . . . . . . . . 111

11 Population Genetics 119

11.1 Data . . . . . . . . . . . . . . . . . . . 119

11.2 Preprocessing . . . . . . . . . . . . . . . . . . . . . . . . . . . . . . . . . . . . . . . . . . . . . .

11.3 Embedding . . . . . . . . . . . . . . . . . 122

12 Single-Cell Genomics $\quad 129$

12.1 Data . . . . . . . . . . . . . . . . . . . . . 129

12.2 Preprocessing . . . . . . . . . . . . . . . . 130 
12.3 Embedding . . . . . . . . . . . . . . . . . 130

13 Conclusions

References 


\section{Abstract}

We consider the vector embedding problem. We are given a finite set of items, with the goal of assigning a representative vector to each one, possibly under some constraints (such as the collection of vectors being standardized, i.e., having zero mean and unit covariance). We are given data indicating that some pairs of items are similar, and optionally, some other pairs are dissimilar. For pairs of similar items, we want the corresponding vectors to be near each other, and for dissimilar pairs, we want the vectors to not be near each other, measured in Euclidean distance. We formalize this by introducing distortion functions, defined for some pairs of items. Our goal is to choose an embedding that minimizes the total distortion, subject to the constraints. We call this the minimum-distortion embedding (MDE) problem.

The MDE framework is simple but general. It includes a wide variety of specific embedding methods, such as spectral embedding, principal component analysis, multidimensional scaling, Euclidean distance problems, dimensionality reduction methods (like Isomap and UMAP), semi-supervised learning, sphere packing, forcedirected layout, and others. It also includes new embeddings, and provides principled ways of validating or sanity-checking historical and new embeddings alike.

In a few special cases, MDE problems can be solved exactly. For others, we develop a projected quasi-Newton method that approximately minimizes the distortion and scales to very large data sets, while placing few assumptions on the distortion functions and constraints. This monograph is accompanied by an opensource Python package, PyMDE, for approximately solving MDE problems. Users can select from a library of distortion functions and constraints or specify custom ones, making it easy to rapidly experiment with new embeddings. Because our algorithm is scalable, and because PyMDE can exploit GPUs, our software scales to problems with millions of items and tens of millions of distortion functions. Additionally, PyMDE is competitive in runtime with specialized implementations of specific embedding methods. To demonstrate our method, we compute embeddings for several real-world data sets, including images, an academic co-author network, US county demographic data, and single-cell mRNA transcriptomes. 


\section{Chapter 1}

\section{Introduction}

An embedding of $n$ items, labeled $1, \ldots, n$, is a function $F$ mapping the set of items into $\mathbf{R}^{m}$. We refer to $x_{i}=F(i)$ as the embedding vector associated with item $i$. In applications, embeddings provide concrete numerical representations of otherwise abstract items, for use in downstream tasks. For example, a biologist might look for subfamilies of related cells by clustering embedding vectors associated with individual cells, while a machine learning practitioner might use vector representations of words as features for a classification task. Embeddings are also used for visualizing collections of items, with embedding dimension $m$ equal to one, two, or three.

For an embedding to be useful, it should be faithful to the known relationships between items in some way. There are many ways to define faithfulness. A working definition of a faithful embedding is the following: if items $i$ and $j$ are similar, their associated vectors $x_{i}$ and $x_{j}$ should be near each other, as measured by the Euclidean distance $\left\|x_{i}-x_{j}\right\|_{2}$; if items $i$ and $j$ are dissimilar, $x_{i}$ and $x_{j}$ should be distant, or at least not close, in Euclidean distance. (Whether two items are similar or dissimilar depends on the application. For example two biological cells might be considered similar if some distance between their mRNA transcriptomes is small.) Many well-known embedding methods like principal component analysis (PCA), spectral embedding [CG97; BN02], and multidimensional scaling [Tor52; Kru64a] use this basic notion of faithfulness, differing in how they make it precise.

The literature on embeddings is both vast and old. PCA originated over a century ago [Pea01], and it was further developed three decades later in the field of psychology [Hot33; EY36]. Multidimensional scaling, a family of methods for embedding items given dissimilarity scores or distances between items, was also developed in the field of psychology during the early-to-mid 20th century [Ric38; Tor52; Kru64a]. Methods for embedding items that are vectors can be traced back to the early 1900s [Men28; 
YH38], and more recently developed methods use tools from convex optimization and convex analysis [BY04; Hay ${ }^{+}$1]. In spectral clustering, an embedding based on an eigenvector decomposition of the graph Laplacian is used to cluster graph vertices [PSL90; vL07]. During this century, dozens of embedding methods have been developed for reducing the dimension of high-dimensional vector data, including Laplacian eigenmaps [BN02], Isomap [TDSL00], locally-linear embedding (LLE) [RS00], stochastic neighborhood embedding (SNE) [HR03], t-distributed stochastic neighbor embedding (t-SNE) [MH08], LargeVis [Tan $\left.{ }^{+} 16\right]$ and uniform manifold approximation and projection (UMAP) [MHM18]. All these methods start with either weights describing the similarity of a pair of items, or distances describing their dissimilarity.

In this monograph we present a general framework for faithful embedding. The framework, which we call minimum-distortion embedding (MDE), generalizes the common cases in which similarities between items are described by weights or distances. It also includes most of the embedding methods mentioned above as special cases. In our formulation, for some pairs of items, we are given distortion functions of the Euclidean distance between the associated embedding vectors. Evaluating a distortion function at the Euclidean distance between the vectors gives the distortion of the embedding for a pair of items. The goal is to find an embedding that minimizes the total or average distortion, possibly subject to some constraints on the embedding; we call this the MDE problem. We focus on three specific constraints: a centering constraint, which requires the embedding to have mean zero, an anchoring constraint, which fixes the positions of a subset of the embedding vectors, and a standardization constraint, which requires the embedding to be centered and have identity covariance.

MDE problems are in general intractable, admitting efficiently computable (global) solutions only in a few special cases like PCA and spectral embedding. In most other cases, MDE problems can only be approximately solved, using heuristic methods. We develop one such heuristic, a projected quasi-Newton method. The method we describe works well for a variety of MDE problems.

This monograph is accompanied by an open-source implementation for specifying MDE problems and computing low-distortion embeddings. Our software package, PyMDE, makes it easy for practitioners to experiment with different embeddings via different choices of distortion functions and constraint sets. Our implementation scales to very large datasets and to embedding dimensions that are much larger than two or three. This means that our package can be used for both visualizing large amounts of data and generating features for downstream tasks. PyMDE supports GPU acceleration and automatic differentiation of distortion functions by using Py- 
Torch $\left[\mathrm{Pas}^{+} 19\right]$ as the numerical backend.

A preview of our framework. Here we give a brief preview of the MDE framework, along with a simple example of an MDE problem. We discuss the MDE problem at length in chapter 2.

An embedding can be represented concretely by a matrix $X \in \mathbf{R}^{n \times m}$, whose rows $x_{1}^{T}, \ldots, x_{n}^{T} \in \mathbf{R}^{m}$ are the embedding vectors. We use $\mathcal{E}$ to denote the set of pairs, and $f_{i j}: \mathbf{R}_{+} \rightarrow \mathbf{R}$ to denote the distortion functions for $(i, j) \in \mathcal{E}$. Our goal is to find an embedding that minimizes the average distortion

$$
E(X)=\frac{1}{|\mathcal{E}|} \sum_{(i, j) \in \mathcal{E}} f_{i j}\left(d_{i j}\right),
$$

where $d_{i j}=\left\|x_{i}-x_{j}\right\|_{2}$, subject to constraints on the embedding, expressed as $X \in \mathcal{X}$, where $\mathcal{X} \subseteq \mathbf{R}^{n \times m}$ is the set of allowable embeddings. Thus the MDE problem is

$$
\begin{array}{ll}
\text { minimize } & E(X) \\
\text { subject to } & X \in \mathcal{X} .
\end{array}
$$

We solve this problem, sometimes approximately, to find an embedding.

An important example is the quadratic MDE problem with standardization constraint. In this problem the distortion functions are quadratic $f_{i j}\left(d_{i j}\right)=w_{i j} d_{i j}^{2}$, where $w_{i j} \in \mathbf{R}$ is a weight conveying similarity (when $w_{i j}>0$ ) or dissimilarity (when $\left.w_{i j}<0\right)$ of items $i$ and $j$. We constrain the embedding $X$ to be standardized, i.e., it must satisfy $(1 / n) X^{T} X=I$ and $X^{T} \mathbf{1}=0$, which forces the embedding vectors to spread out. While most MDE problems are intractable, the quadratic MDE problem is an exception: it admits an analytical solution via eigenvectors of a certain matrix. Many well-known embedding methods, including PCA, spectral embedding, and classical multidimensional scaling, are instances of quadratic MDE problems, differing only in their choice of pairs and weights. Quadratic MDE problems are discussed in chapter 3 .

Why the Euclidean norm? A natural question is why we use the Euclidean norm as our distance measure between embedding vectors. First, when we are embedding into $\mathbf{R}^{2}$ or $\mathbf{R}^{3}$ for the purpose of visualization or discovery, the Euclidean distance corresponds to actual physical distance, making it a natural choice. Second, it is traditional, and follows a large number of known embedding methods like PCA and spectral embedding that also use Euclidean distance. Third, the standardization constraint we consider in this monograph has a natural interpretation when we use 
the Euclidean distance, but would make little sense if we used another metric. Finally, we mention that the local optimization methods described in this monograph can be easily extended to the case where distances between embedding vectors are measured with a non-Euclidean metric.

\subsection{Contributions}

The main contributions of this monograph are the following:

1. We present a simple framework, MDE, that unifies and generalizes many different embedding methods, both classical and modern. This framework makes it easier to interpret existing embedding methods and to create new ones. It also provides principled ways to validate, or at least sanity-check, embeddings.

2. We develop an algorithm for approximately solving MDE problems (i.e., for computing embeddings) that places very few assumptions on the distortion functions and constraints. This algorithm reliably produces good embeddings in practice and scales to large problems.

3. We provide open-source software that makes it easy for users to solve their own MDE problems and obtain custom embeddings. Our implementation of our solution method is competitive in runtime to specialized algorithms for specific embedding methods.

\subsection{Outline}

This monograph is divided into three parts, I Minimum-Distortion Embedding, II Algorithms, and III Examples.

Part I: Minimum-distortion embedding. We begin part I by describing the MDE problem and some of its properties in chapter 2. We introduce the notion of anchored embeddings, in which some of the embedding vectors are fixed, and standardized embeddings, in which the embedding vectors are constrained to have zero mean and identity covariance. Standardized embeddings are favorably scaled for many tasks, such as for use as features for supervised learning.

In chapter 3 we study MDE problems with quadratic distortion, focusing on the problems with a standardization constraint. This class of problems has an analytical solution via an eigenvector decomposition of a certain matrix. We show that many 
existing embedding methods, including spectral embedding, PCA, Isomap, kernel PCA, and others, reduce to solving instances of the quadratic MDE problem.

In chapter 4 we describe examples of distortion functions, showing how different notions of faithfulness of an embedding can be captured by different distortion functions. Some choices of the distortion functions (and constraints) lead to MDE problems solved by well-known methods, while others yield MDE problems that, to the best of our knowledge, have not appeared elsewhere in the literature.

Part II: Algorithms. In part II, we describe algorithms for computing embeddings. We begin by presenting stationarity conditions for the MDE problem in chapter 5 , which are necessary but not sufficient for an embedding to be optimal. The stationarity conditions have a simple form: the gradient of the average distortion, projected onto the set of tangents of the constraint set at the current point, is zero. This condition guides our development of algorithms for computing embeddings.

In chapter 6 , we present a projected quasi-Newton algorithm for approximately solving MDE problems. For very large problems, we additionally develop a stochastic proximal algorithm that uses the projected quasi-Newton algorithm to solve a sequence of smaller regularized MDE problems. Our algorithms can be applied to MDE problems with differentiable average distortion, and any constraint set for which there exists an efficient projection onto the set and an efficient projection onto the set of tangents of the constraint set at the current point. This includes MDE problems with centering, anchor, or standardization constraints.

In chapter 7 , we present numerical examples demonstrating the performance of our algorithms. We also describe a software implementation of these methods, and briefly describe our open-source implementation PyMDE.

Part III: Examples. In part III, we use PyMDE to approximately solve many MDE problems involving real datasets, including images (chapter 8), co-authorship networks (chapter 9), United States county demographics (chapter 10), population genetics (chapter 11), and single-cell mRNA transcriptomes (chapter 12).

\subsection{Related work}

Dimensionality reduction. In many applications, the original items are associated with high-dimensional vectors, and we can interpret the embedding into the smaller dimensional space as dimensionality reduction. Dimensionality reduction can 
be used to reduce the computational burden of numerical tasks, compared to carrying them out with the original high-dimensional vectors. When the embedding dimension is two or three, dimension reduction can also be used to visualize the original high-dimensional data and facilitate exploratory data analysis. For example, visualization is an important first step in studying single-cell mRNA transcriptomes, a relatively new type of data in which each cell is represented by a high-dimensional vector encoding gene expression [San14; KB19].

Dozens of methods have been developed for dimensionality reduction. PCA, the Laplacian eigenmap [BN02], Isomap [TDSL00], LLE [RS00], maximum variance unfolding [WS04], t-SNE [MH08], LargeVis [Tan+16], UMAP [MHM18], and the latent variable model (LVM) from [Sau20] are all dimensionality reduction methods. With the exception of t-SNE and the LVM, these methods can be interpreted as solving different MDE problems, as we will see in chapters 3 and 4. We exclude t-SNE because its objective function is not separable in the embedding distances; however, methods like LargeVis and UMAP have been observed to produce embeddings that are similar to t-SNE embeddings [BBK20]. We exclude the LVM because it fits some parameters in addition to the embedding.

Dimensionality reduction is sometimes called manifold learning in the machine learning community, since some of these methods can be motivated by a hypothesis that the original data lie in a low-dimensional manifold, which the dimensionality reduction method seeks to recover [MF11; Cay05; LZ08; Wil ${ }^{+} 14$; NK17].

Finally, we note that dimensionality reduction methods have been studied under general frameworks other than MDE $\left[\mathrm{Ham}^{+} 04 ; \mathrm{Yan}^{+} 06\right.$; KCS11; Law11; $\left.\mathrm{Wan}^{+} 20\right]$.

Metric embedding. Another well-studied class of embeddings are those that embed one finite metric space into another one. There are many ways to define the distortion of such an embedding. One common definition is the maximum fractional error between the embedding distances and original distances, across all pairs of items. (This can be done by insisting that the embedding be non-contractive, i.e., the embedding distances are at least the original distances, and then minimizing the maximum ratio of embedding distance to original distance.)

An important result in metric embedding is the Johnson-Lindenstrauss Lemma, which states that a linear map can be used to reduce the dimension of vector data, scaling distances by no more than $(1 \pm \epsilon)$, when the target dimension $m$ is $O\left(\log n / \epsilon^{2}\right)$ [JL84]. Another important result is due to Bourgain, who showed that any finite metric can be embedded in Euclidean space with at most a logarithmic distortion [Bou85]. A constructive method via semidefinite programming was later developed [LLR95]. Several other results, including impossibility results, have been discovered 
[IMS17], and some recent research has focused on embedding into non-Euclidean spaces, such as hyperbolic space [Sal $\left.{ }^{+} 18\right]$.

In this monograph, for some of the problems we consider, all that is required is to place similar items near each other, and dissimilar items not near each other; in such applications we may not even have original distances to preserve. In other problems we do start with original distances. In all cases we are interested in minimizing an average of distortion functions (not maximum), which is more relevant in applications, especially since real-world data is noisy and may contain outliers.

Force-directed layout. Force-directed methods are algorithms for drawing graphs in the plane in an aesthetically pleasing way. In a force-directed layout problem, the vertices of the graph are considered to be nodes connected by springs. Each spring exerts attractive or repulsive forces on the two nodes it connects, with the magnitude of the forces depending on the Euclidean distance between the nodes. Force-directed methods move the nodes until a static equilibrium is reached, with zero net force on each node, yielding an embedding of the vertices into $\mathbf{R}^{2}$. Force-directed methods, which are also called spring embedders, can be considered as MDE problems in which the distortion functions give the potential energy associated with the springs. Force-directed layout is a decades-old subject [Tut63; Ead84; KK89], with early applications in VLSI layout [FCW67; QB79] and continuing modern interest [Kob12].

Low-rank models. A low-rank model approximates a matrix by one of lower rank, typically factored as the product of a tall and a wide matrix. These factors can be interpreted as embeddings of the rows and columns of the original matrix. Well-known examples of low-rank models include PCA and non-negative matrix factorization [LS99]; there are many others [Ude $\left.{ }^{+} 16, \S 3.2\right]$. PCA (and its kernelized version) can be interpreted as solving an MDE problem, as we show in $§ 3.2$.

X2vec. Embeddings are frequently used to produce features for downstream machine learning tasks. Embeddings for this purpose were popularized with the publication of word2vec in 2013, an embedding method in which the items are words $\left[\mathrm{Mik}^{+} 13\right]$. Since then, dozens of embeddings for different types of items have been proposed, such as doc2vec [LM14], node2vec [GL16] and related methods [PARS14; $\mathrm{Tan}^{+}$15], graph2vec [Nar ${ }^{+}$17], role2vec [Ahm ${ }^{+}$20], (batter-pitcher)2vec [Alc16], BioVec, ProtVec, and GeneVec [AM15], dna2vec [Ng17], and many others. Some of these methods resemble MDE problems, but most of them do not. Nonetheless MDE problems generically can be used to produce such X2vec-style embeddings, where X describes the type of items. 
Neural networks. Neural networks are commonly used to generate embeddings for use in downstream machine learning tasks. One generic neural network based embedding method is the auto-encoder, which starts by representing items by (usually large dimensional) input vectors, such as one-hot vectors. These vectors are fed into an encoder neural network, whose output is fed into a decoder network. The output of the encoder has low dimension, and will give our embedding. The decoder attempts to reconstruct the original input from this low-dimensional intermediate vector. The encoder and decoder are both trained so the decoder can, at least approximately, reproduce the original input [GBC16, §14].

More generally, a neural network may be trained to predict some relevant quantity, and the trained network's output (or an intermediate activation) can be used as the input's embedding. For example, neural networks for embedding words (or sequences of words) are often trained to predict masked words in a sentence; this is the basic principle underlying word2vec and BERT, two well-known word embedding methods $\left[\mathrm{Mik}^{+} 13 ; \mathrm{Dev}^{+} 19\right]$. Similarly, intermediate activations of convolutional neural networks like residual networks $\left[\mathrm{He}^{+} 16\right]$, trained to classify images, are often used as embeddings of images. Neural networks have also been used for embedding single-cell mRNA transcriptomes [ $\left.\mathrm{Szu}^{+} 19\right]$.

Software. There are several open-source software libraries for specific embedding methods. The widely used Python library sci-kit learn $\left[\mathrm{Ped}^{+} 11\right]$ includes implementations of PCA, spectral embedding, Isomap, locally linear embedding, multidimensional scaling, and t-SNE, among others. The umap-learn package implements UMAP [McI20b], the openTSNE package provides a more scalable variant of t-SNE [PSZ19], and GraphVite (which can exploit multiple CPUs and GPUs) implements a number of embedding methods [Zhu+19]. Embeddings for words and documents are available in gensim [ŘS10], Embeddings.jl [WE19], HuggingFace transformers [Hug20], and BERT [Dev20]. Force-directed layout methods are implemented in graphviz [GN00], NetworkX [HSS08], qgraph [Eps $\left.{ }^{+} 12\right]$, and NetworkLayout.jl [Net].

There are also several software libraries for approximately solving optimization problems with orthogonality constraints (which the MDE problem with standardization constraint has). Some examples include Manopt (and its related packages PyManopt and Manopt.jl) [Bou ${ }^{+} 14$; TKW16; Ber20a], Geoopt [KKK20], and McTorch $\left[\mathrm{Meg}^{+} 18\right]$. More generally, problems with differentiable objective and constraint functions can be approximately solved using solvers for nonlinear programming, such as SNOPT [GMS02] (which is based on sequential quadratic programming) and IPOPT [WB06] (which is based on an interior-point method). 


\section{Part I}

\section{Minimum-Distortion Embedding}




\section{Chapter 2}

\section{Minimum-Distortion Embedding}

In this chapter we introduce the minimum-distortion embedding problem and explore some of its general properties.

\subsection{Embedding}

We start with a finite set of items $\mathcal{V}$, which we label as $1, \ldots, n$, so $\mathcal{V}=\{1, \ldots, n\}$. An embedding of the set of items $\mathcal{V}$ into $\mathbf{R}^{m}$ is a function $F: \mathcal{V} \rightarrow \mathbf{R}^{m}$. We denote the values of $F$ by $x_{i}=F(i), i=1, \ldots, n$, and denote the embedding concretely by a matrix $X \in \mathbf{R}^{n \times m}$,

$$
X=\left[\begin{array}{c}
x_{1}^{T} \\
x_{2}^{T} \\
\vdots \\
x_{n}^{T}
\end{array}\right] .
$$

The rows of $X$ are the transposes of the vectors associated with the items $1, \ldots, n$. The columns of $X$ can be interpreted as $m$ features or attributes of the items, with $X_{i j}=\left(x_{i}\right)_{j}$ the value of $j$ th feature or attribute for item $i$; the $j$ th column of $X$ gives the values of the $j$ th feature assigned to the $n$ items.

Throughout this monograph, the quality of an embedding $X$ will only depend on the embedding distances $d_{i j}$ between items $i$ and $j$, defined as

$$
d_{i j}=\left\|x_{i}-x_{j}\right\|_{2}, \quad i, j=1, \ldots, n,
$$

i.e., $d_{i j}$ is the Euclidean distance between the vectors $x_{i}$ and $x_{j}$. Embedding distances are evidently not affected by translation of the embedded points, i.e., replacing each $x_{i}$ by $x_{i}+a$, where $a \in \mathbf{R}^{m}$, or by orthogonal transformation, i.e., replacing each 
$x_{i}$ by $Q x_{i}$, where $Q$ is an orthogonal $m \times m$ matrix. If there are no constraints on the embedding vectors, then without any loss of generality we can assume that the average of the $x_{i}$ is zero, or equivalently, $X^{T} \mathbf{1}=0$, where $\mathbf{1}$ is the vector with all entries one. This means that each of the $m$ features (columns of $X$ ) has mean value zero across our $n$ items.

\subsection{Distortion}

We express our desires about the embedding distances by distortion functions associated with embedding distances. These have the form

$$
f_{i j}: \mathbf{R}_{+} \rightarrow \mathbf{R}
$$

for $(i, j) \in \mathcal{E}$, where $\mathcal{E} \subseteq \mathcal{V} \times \mathcal{V}$ is the set of item pairs for which we have an associated function. We will assume that $\mathcal{E}$ is nonempty, and that $i<j$ for every $(i, j) \in \mathcal{E}$.

The value $f_{i j}\left(d_{i j}\right)$ is the distortion for the pair $(i, j) \in \mathcal{E}$ : the smaller $f_{i j}\left(d_{i j}\right)$ is, the better the embedding captures the relationship between items $i$ and $j$. We can interpret these distortion functions as the weights of a generalized graph $(\mathcal{V}, \mathcal{E})$, in which the edge weights are functions, not scalars.

We will assume that the distortion functions $f_{i j}$ are differentiable. As in many other applications, however, we have observed that the algorithm we propose, which assumes differentiability, works well in practice even when the distortion functions are not differentiable.

\subsubsection{Examples}

The distortion functions $f_{i j}$ we will encounter typically derive from given weights (or similarities) associated with pairs of items, or from associated deviations (or distances or dissimilarities) between items, or both. Distortion functions can be also derived from one or more relations (or undirected graphs) on the items. Below, we give simple examples of such distortion functions. We give many more examples in chapter 4.

Distortion functions derived from weights. We start with a set of nonzero weights $w_{i j} \in \mathbf{R}$, for $(i, j) \in \mathcal{E}$. The larger $w_{i j}$, the more similar items $i$ and $j$ are; negative weights indicate dissimilarity. We partition the edges into those associated with positive weights (similar items) and negative weights (dissimilar items), with

$$
\mathcal{E}_{\text {sim }}=\left\{(i, j) \mid w_{i j}>0\right\}, \quad \mathcal{E}_{\text {dis }}=\left\{(i, j) \mid w_{i j}<0\right\},
$$


so $\mathcal{E}=\mathcal{E}_{\text {sim }} \cup \mathcal{E}_{\text {dis }}$ and $\mathcal{E}_{\text {sim }} \cap \mathcal{E}_{\text {dis }}=\emptyset$.

Distortion functions derived from weights have the form

$$
f_{i j}\left(d_{i j}\right)= \begin{cases}w_{i j} p_{\mathrm{s}}\left(d_{i j}\right), & (i, j) \in \mathcal{E}_{\mathrm{sim}} \\ w_{i j} p_{\mathrm{d}}\left(d_{i j}\right), & (i, j) \in \mathcal{E}_{\mathrm{dis}}\end{cases}
$$

where $p_{\mathrm{s}}$ and $p_{\mathrm{d}}$ are penalty functions associated with positive weights (similar items) and negative weights (dissimilar items), respectively. The penalty functions are increasing, so $f_{i j}$ is increasing when $w_{i j}$ is positive and decreasing when $w_{i j}$ is negative. Roughly speaking, the closer vectors associated with similar items are, and the farther vectors associated with dissimilar items are, the lower the distortion. The weights $w_{i j}$, which indicate the degree of similarity or dissimilarity, scale the penalty functions.

Perhaps the simplest distortion function derived from weights is the quadratic

$$
f_{i j}\left(d_{i j}\right)=w_{i j} d_{i j}^{2},
$$

for which $p_{\mathrm{s}}\left(d_{i j}\right)=p_{\mathrm{d}}\left(d_{i j}\right)=d_{i j}^{2}$. The quadratic distortion is plotted in figure 2.1, for weights $w_{i j}=1$ and $w_{i j}=-1$. For $w_{i j}=1$, which means items $i$ and $j$ are similar, the distortion rises as the distance between $x_{i}$ and $x_{j}$ increases; for $w_{i j}=-1$, which means items $i$ and $j$ are dissimilar, the distortion is negative and becomes more negative as the distance between $x_{i}$ and $x_{j}$ increases. We will study the quadratic penalty in chapter 3 .

Distortion functions derived from deviations. We start with nonnegative numbers $\delta_{i j}$ for $(i, j) \in \mathcal{E}$ that represent deviations or (original) distance between items $i$ and $j$, with small $\delta_{i j}$ meaning the items are similar and large $\delta_{i j}$ meaning the items are dissimilar. The smaller $\delta_{i j}$, the more similar items $i$ and $j$ are. The original deviation data $\delta_{i j}$ need not be a metric.

Distortion functions derived from deviations or distances have the general form

$$
f_{i j}\left(d_{i j}\right)=\ell\left(\delta_{i j}, d_{i j}\right),
$$

where $\ell$ is a loss function, which is nonnegative, with $\ell\left(\delta_{i j}, \delta_{i j}\right)=0$, decreasing in $d_{i j}$ for $d_{i j}<\delta_{i j}$ and increasing for $d_{i j}>\delta_{i j}$. We can interpret the given deviation $\delta_{i j}$ as a target or desired value for the embedding distance $d_{i j}$; the distortion $\ell\left(\delta_{i j}, d_{i j}\right)$ measures the difference between the target distance and the embedding distance.

The simplest example uses a square loss,

$$
f_{i j}\left(d_{i j}\right)=\left(\delta_{i j}-d_{i j}\right)^{2},
$$




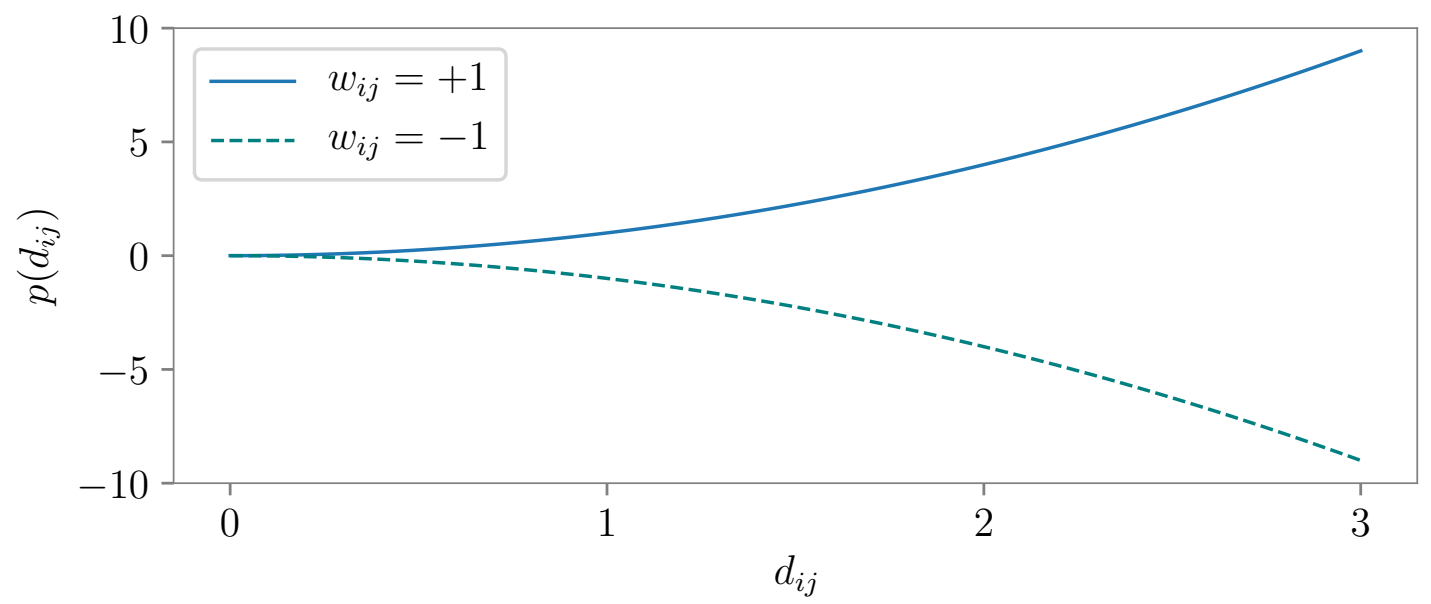

Figure 2.1: Quadratic penalties, with $w_{i j}=+1$ or $w_{i j}=-1$ for $(i, j) \in \mathcal{E}$.

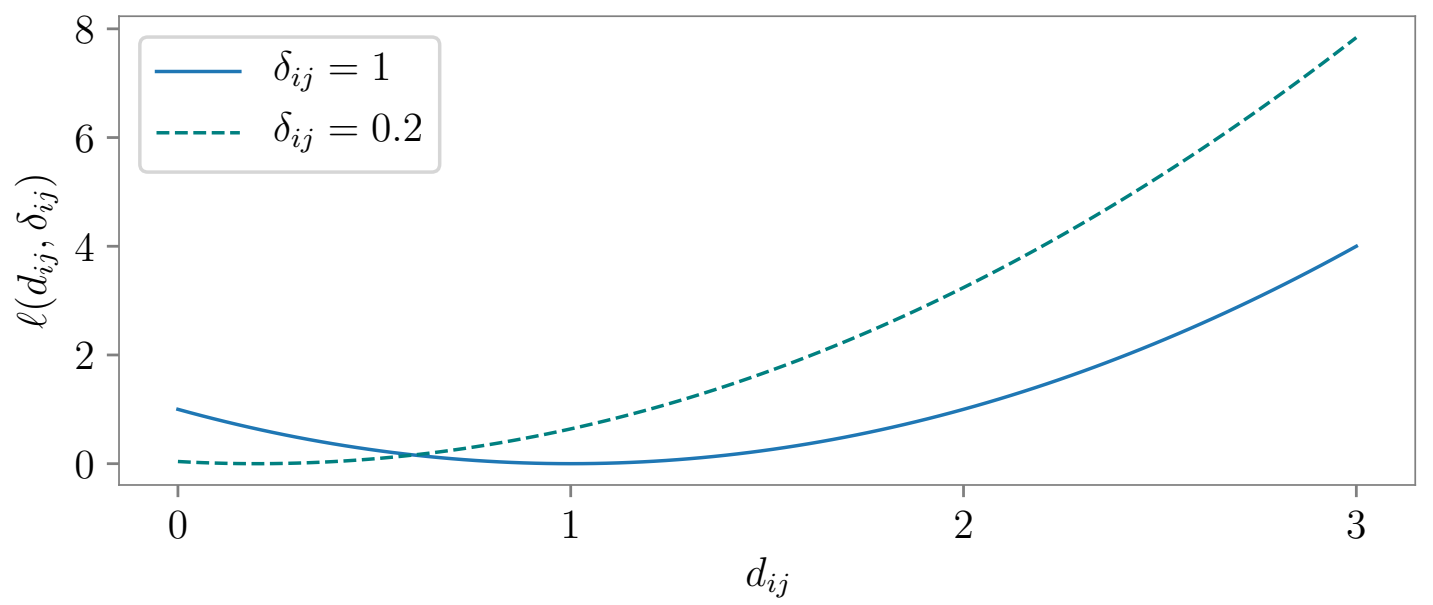

Figure 2.2: Quadratic losses, with $\delta_{i j}=1$ or $\delta_{i j}=0.2$ for $(i, j) \in \mathcal{E}$. 
the square of the difference between the given deviation and the embedding distance. Distortions derived from the square loss and deviations are shown in figure 2.2, for $\delta_{i j}=0.2$ and $\delta_{i j}=1$. For original deviation $\delta_{i j}=1$, the distortion has its minimum value at $d_{i j}=1$, i.e., when the distance between $x_{i}$ and $x_{j}$ is one. It rises as $d_{i j}$ deviates from the target $\delta_{i j}=1$.

Distortion functions derived from a graph. In some applications we start with an undirected graph or a relation on the items, i.e., a set of pairs $\mathcal{E}$. The associated distortion function is

$$
f_{i j}\left(d_{i j}\right)=p\left(d_{i j}\right), \quad(i, j) \in \mathcal{E},
$$

where $p$ is a penalty function, which is increasing. Such distortion functions can be interpreted as deriving from weights or deviations, in the special case when there is only one value for the weights or deviations. The simplest such distortion function is quadratic, with $p\left(d_{i j}\right)=d_{i j}^{2}$.

Distortion functions derived from multiple graphs. As a variation, we can have multiple original graphs or relations on the items. For example suppose we have $\mathcal{E}_{\text {sim }}$, a set of pairs of items that are similar, and also $\mathcal{E}_{\text {dis }}$, a set of pairs of items that are dissimilar, with $\mathcal{E}_{\text {sim }} \cap \mathcal{E}_{\text {dis }}=\emptyset$. From these two graphs we can derive the distortion functions

$$
f_{i j}\left(d_{i j}\right)= \begin{cases}p_{\mathrm{s}}\left(d_{i j}\right) & (i, j) \in \mathcal{E}_{\mathrm{sim}} \\ -p_{\mathrm{d}}\left(d_{i j}\right) & (i, j) \in \mathcal{E}_{\mathrm{dis}},\end{cases}
$$

with $\mathcal{E}=\mathcal{E}_{\text {sim }} \cup \mathcal{E}_{\text {dis }}$, where $p_{\mathrm{s}}$ and $p_{\mathrm{d}}$ are increasing penalty functions for similar and dissimilar pairs, respectively. This is a special case of distortion functions described by weights, when the weights take on only two values, +1 (for similar items) and -1 (for dissimilar items).

Connections between weights, deviations, and graphs. The distinction between distortion functions derived from weights, deviations, or graphs is not sharp. As a specific example, suppose we are given some original deviations $\delta_{i j}$, and we take

$$
f_{i j}\left(d_{i j}\right)=\left\{\begin{array}{ll}
d_{i j}^{2} & \delta_{i j} \leq \epsilon \\
0 & \text { otherwise },
\end{array} \quad(i, j) \in \mathcal{E},\right.
$$

where $\epsilon$ is a given positive threshold. This natural distortion function identifies an unweighted graph of neighbors (defined by small original distance or deviation), and puts unit weight quadratic distortion on those edges. (We could just as well have 
started with original similarities or weights $w_{i j}$ and constructed the neighbor graph as $w_{i j} \geq \epsilon$.) This distortion evidently combines the ideas of distances, weights, and graphs.

\subsubsection{Average distortion}

We evaluate the quality of an embedding by its average distortion, defined as

$$
E(X)=\frac{1}{|\mathcal{E}|} \sum_{(i, j) \in \mathcal{E}} f_{i j}\left(d_{i j}\right) .
$$

The smaller the average distortion, the more faithful the embedding. In chapter 4, we will see that a wide variety of notions of faithfulness of an embedding can be captured by different choices of the distortion functions $f_{i j}$. (Our use of the symbol $E$ to denote average distortion is meant to vaguely hint at energy, from a mechanical interpretation we will see later.)

The average distortion $E(X)$ models our displeasure with the embedding $X$. With distortion functions defined by weights, small $E(X)$ corresponds to an embedding with vectors associated with large (positive) weights (i.e., similar items) typically near each other, and vectors associated with negative weights (i.e., dissimilar items) not near each other. For distortion functions defined by deviations, small $E(X)$ means that the Euclidean distances between vectors are close to the original given deviations.

Evidently, $(i, j) \notin \mathcal{E}$ means that $E(X)$ does not depend on $d_{i j}$. We can interpret $(i, j) \notin \mathcal{E}$ in several related ways. One interpretation is that we are neutral about the distance $d_{i j}$, that is, we do not care if it is small or large. Another interpretation is that the data required to specify the distortion $f_{i j}$, such as a weight $w_{i j}$ or an original distance $\delta_{i j}$, is not available.

\subsection{Minimum-distortion embedding}

We propose choosing an embedding $X \in \mathbf{R}^{n \times m}$ that minimizes the average distortion, subject to $X \in \mathcal{X}$, where $\mathcal{X}$ is the set of feasible or allowable embeddings. This gives the optimization problem

$$
\begin{array}{ll}
\text { minimize } & E(X) \\
\text { subject to } & X \in \mathcal{X},
\end{array}
$$

with optimization variable $X \in \mathbf{R}^{n \times m}$. We refer to this problem as the minimumdistortion embedding (MDE) problem, and we call a solution to this problem a minimum-distortion embedding. 
In this monograph we will focus on three specific constraint sets $\mathcal{X}$ : the set of embeddings centered at the origin, the set of embeddings in which some of the embedding vectors are anchored (fixed) to specific values, and the set of standardized embeddings, which are centered embeddings with unit covariance. These constraint sets are described in $\S 2.4$.

MDE problems can be solved exactly only in a few special cases, one of which is described in chapter 3. In most other cases, solving an MDE problem exactly is intractable, so in chapter 6 we propose heuristic methods to solve it approximately. In our experience, these methods often find low-distortion embeddings and scale to large datasets (chapter 7). With some abuse of language, we refer to an embedding that is an approximate solution of the MDE problem as a minimum-distortion embedding, even if it does not globally solve (2.1).

With our assumption that the distortion functions are differentiable, the average distortion $E$ is differentiable, provided the embedding vectors are distinct, i.e., $x_{i} \neq$ $x_{j}$ for $(i, j) \in \mathcal{E}, i \neq j$, which implies $d_{i j}>0$ for $(i, j) \in \mathcal{E}$. It is differentiable even when $d_{i j}=0$, provided $f_{i j}^{\prime}(0)=0$. For example, $E$ is differentiable (indeed, quadratic) when the distortion functions are quadratic.

The MDE problem (2.1) admits multiple interesting interpretations. Thinking of the problem data as a generalized weighted graph, a minimum-distortion embedding can be interpreted as a representation of the vertices by vectors that respects the geometry of the graph, connecting our problem to long lines of work on graph embeddings [LLR95; Yan ${ }^{+}$06; CG97; HYL17]. The MDE problem can also be given a mechanical interpretation, connecting it to force-directed layout [Ead84].

Mechanical interpretation. In the mechanical interpretation, we consider $x_{i}$ to be a point in $\mathbf{R}^{m}$ associated with item $i$. We imagine each pair of points $(i, j) \in \mathcal{E}$ as connected by a spring with potential energy function $f_{i j}$, i.e., $f_{i j}\left(d_{i j}\right)$ is the elastic stored energy in the spring when it is extended a distance $d_{i j}$. The spring associated with $(i, j) \in \mathcal{E}$ has a tension force versus extension given by $f_{i j}^{\prime}$. A force with this magnitude is applied to both points $i$ and $j$, each in the direction of the other. Thus the spring connecting $x_{i}$ and $x_{j}$ contributes the (vector) forces

$$
f_{i j}^{\prime}\left(d_{i j}\right) \frac{x_{j}-x_{i}}{\left\|x_{i}-x_{j}\right\|_{2}}, \quad-f_{i j}^{\prime}\left(d_{i j}\right) \frac{x_{j}-x_{i}}{\left\|x_{i}-x_{j}\right\|_{2}},
$$

on the points $x_{i}$ and $x_{j}$, respectively. When $f_{i j}^{\prime}$ is positive, the force is attractive, i.e., it pulls the points $x_{i}$ and $x_{j}$ toward each other. When $f_{i j}^{\prime}$ is negative, the force is repulsive, i.e., it pushes the points $x_{i}$ and $x_{j}$ away from each other. 

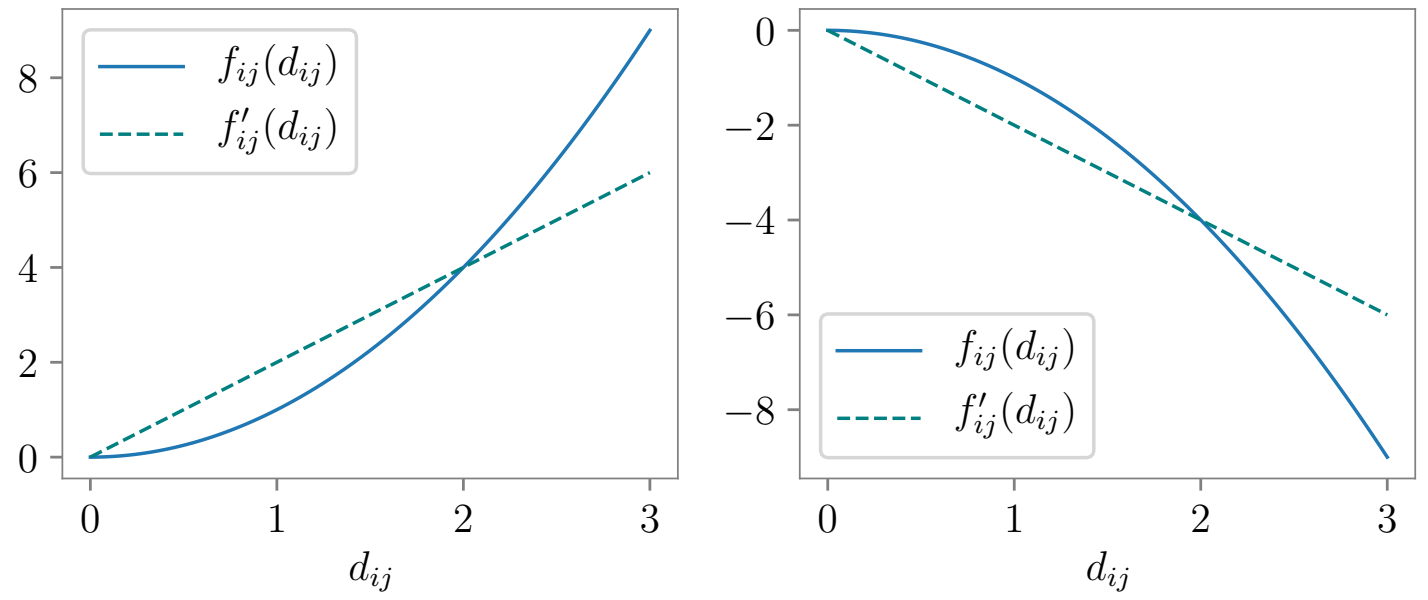

Figure 2.3: Potential energy and force magnitude for quadratic distortion functions. Left. An attractive distortion function. Right. A repulsive distortion function.

Distortion function derived from weights are always attractive for positive weights (pairs of similar items), and always repulsive for negative weights (pairs of dissimilar items). Distortion functions derived from deviations are attractive when $d_{i j}>\delta_{i j}$ and repulsive when $d_{i j}<\delta_{i j}$. For such distortion functions, we can think of $\delta_{i j}$ as the natural length of the spring, i.e., the distance at which it applies no forces on the points it connects. Figure 2.3 shows the potential energy and force for quadratic distortion functions, with weight +1 (left) and -1 (right). Figure 2.4 shows the potential energy and force for a function associated with a quadratic loss.

The MDE objective $E(X)$ is the average elastic stored energy in all the springs. The MDE problem is to find a minimum total potential energy configuration of the points, subject to the constraints. When the constraint is only $X^{T} \mathbf{1}=0$, such a configuration corresponds to one in which the net force on each point, from its neighbors, is zero. (Such a point corresponds to a mechanical equilibrium, not necessarily one of minimum energy.) When there are constraints beyond $X^{T} \mathbf{1}=0$, the mechanical interpretation is a bit more complicated, since the constraints also contribute an additional force on each point, related to an optimal dual variable for the constraint.

Single-index notation. To simplify the notation, we will sometimes use a single index $k$ instead of a tuple of indices $(i, j)$ to represent a pair of items. Specifically, we impose an ordering on the tuples $(i, j) \in \mathcal{E}$, labeling them $1, \ldots, p$, where $p=|\mathcal{E}|$ is 


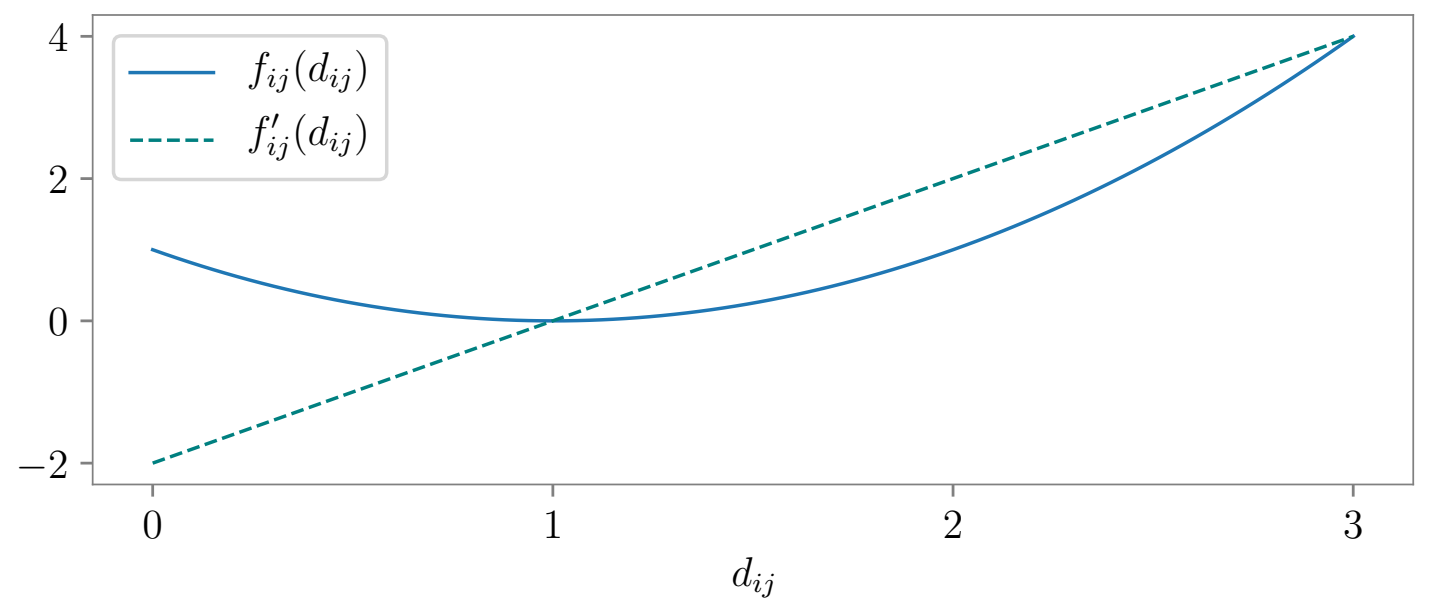

Figure 2.4: Potential energy and force magnitude for a distortion function that is attractive for $d_{i j}>1$ and repulsive for $d_{i j}<1$.

the number of pairs for which distortion functions are given. For a pair $k \in\{1, \ldots, p\}$, we write $i(k)$ to denote its first item and $j(k)$ to denote the second item. We use $d_{k}$ to denote the embedding distance for the $k$ th pair,

$$
d_{k}=\left\|x_{i(k)}-x_{j(k)}\right\|_{2}, \quad k=1, \ldots, p,
$$

with $d \in \mathbf{R}^{p}$ the vector of embedding distances. Similarly, we define the vector distortion function $f: \mathbf{R}^{p} \rightarrow \mathbf{R}^{p}$, whose components are the scalar distortion functions $f_{k}, k=1, \ldots, p$. Using this notation, the average distortion is

$$
E(X)=\frac{1}{p} \sum_{k=1}^{p} f_{k}\left(d_{k}\right)=\mathbf{1}^{T} f(d) / p .
$$

We note for future reference that $p \leq n(n-1) / 2$, with equality when the graph is full. For a connected graph, we have $p \geq n-1$, with equality occurring when the graph is a chain. In many applications the typical degree is small, in which case $p$ is a small multiple of $n$. 
Incidence matrix. Since the problem data can be interpreted as a graph, we can associate with it an incidence matrix $A \in \mathbf{R}^{n \times p}$ [BV18, §7.3], defined as

$$
A_{i j}= \begin{cases}1 & (i, j) \in \mathcal{E} \\ -1 & (j, i) \in \mathcal{E} \\ 0 & \text { otherwise }\end{cases}
$$

(Recall that we assume $i<j$ for each $(i, j) \in \mathcal{E}$.) The $k$ th column of $A$, which we denote $a_{k}$, is associated with the $k$ th pair or edge. Each column has exactly two non-zero entries, with values \pm 1 , that give the items connected by the edge. We can express $a_{k}$ as $a_{k}=e_{i(k)}-e_{j(k)}$, where $e_{l}$ is the $l$ th unit vector in $\mathbf{R}^{n}$.

We can express the embedding distances compactly using the incidence matrix. Using single-index notation, the $k$ th distance $d_{k}$ can be written as

$$
d_{k}=\left\|X^{T} a_{k}\right\|_{2},
$$

or equivalently

$$
d_{k}=\sqrt{\left(A^{T} X X^{T} A\right)_{k k}} .
$$

We mention briefly that the MDE problem can be alternatively parametrized via the Gram matrix $G=X X^{T}$, since $d_{k}=\sqrt{a_{k}^{T} G a_{k}}$. For some constraint sets $\mathcal{X}$, the resulting rank-constrained optimization problem can be approximately solved by methods that manipulate the square root of $G$, using techniques introduced in [BM03; BM05]. We will not use this parametrization in the sequel.

\subsection{Constraints}

\subsubsection{Centered embeddings}

We say that an MDE problem is centered or unconstrained when the constraint set is $\mathcal{X}=\mathcal{C}$, where

$$
\mathcal{C}=\left\{X \mid X^{T} \mathbf{1}=0\right\}
$$

is the set of centered embeddings. (Recall that the constraint $X^{T} \mathbf{1}=0$ is without loss of generality.) An unconstrained MDE problem can fail to be well-posed, i.e., it might not have a solution or might admit trivial solutions.

When an unconstrained MDE problem does have a solution, it is never unique, since both the constraint set $\mathcal{C}$ and the objective $E$ are invariant under rotations. If $X^{\star}$ is any optimal embedding, then $X=X^{\star} Q$ is also optimal, for any orthogonal $m \times m$ matrix $Q$. 


\subsubsection{Anchored embeddings}

In an anchored MDE problem, some of the vectors $x_{i}$ are known and fixed, with

$$
x_{i}=x_{i}^{\text {given }}, \quad i \in \mathcal{K},
$$

where $\mathcal{K} \subseteq \mathcal{V}$ is the set of indices of vectors that are fixed (or anchored), and

$x_{i}^{\text {given }} \in \mathbf{R}^{m}$ are the given values for those vectors. We call (2.4) an anchor constraint; the items with vertices in $\mathcal{K}$ are anchored, and the remaining are free.

We will use $\mathcal{A}$ to represent a set of anchored embeddings, i.e.

$$
\mathcal{A}=\left\{X \mid x_{i}=x_{i}^{\text {given }}, i \in \mathcal{K}\right\} .
$$

(The constraint set $\mathcal{A}$ depends on $\mathcal{K}$ and $x_{i}^{\text {given }}$, but we suppress this dependence in our notation to keep it simple.) Unlike centered embeddings, the constraint set $\mathcal{A}$ is not closed under orthogonal transformations. In particular, MDE problems with this constraint are neither translation nor rotation invariant.

An anchor constraint has a natural mechanical interpretation: the anchored vertices are physically fixed to given positions (as if by nails). We will later describe the force that the nails exert on their associated anchored points. Not surprisingly, in an optimal anchored embedding, the net force on each point (due to its neighbors and its nail, if it is anchored) is zero.

Incremental embedding. Anchor constraints can be used to build an embedding incrementally. In an incremental embedding, we start with an embedding of $X \in$ $\mathbf{R}^{n \times m}$ of a set of items $\mathcal{V}=\{1, \ldots, n\}$. We later obtain a few additional items $\mathcal{V}^{\prime}=\left\{n+1, \ldots, n+n^{\prime}\right\}$ (with $\left.n^{\prime} \ll n\right)$, along with a set of pairs $\mathcal{E}^{\prime} \subseteq\left(\mathcal{V} \cup \mathcal{V}^{\prime}\right) \times$ $\left(\mathcal{V} \cup \mathcal{V}^{\prime}\right)$ and their associated distortion functions relating old items to new items (and possibly relating the new items to each other), i.e., $(i, j) \in \mathcal{E}^{\prime}$ implies $i \in \mathcal{V}$ and $j \in \mathcal{V}^{\prime}$, or $i, j \in \mathcal{V}^{\prime}$ (and $i<j$ ). We seek a new embedding $X^{\prime} \in \mathbf{R}^{n+n^{\prime} \times m}$ in which the first $n$ embedding vectors match the original embedding, and the last $n+1$ embedding vectors correspond to the new items. Such an embedding is readily found by anchoring the first $n$ embedding vectors to their original values, leaving only the last $n+1$ embedding vectors free, and minimizing the average distortion. There are two approaches to handling centering or standardization constraints in incremental embedding. The simplest is to simply ignore these constraints, which is justified when $n^{\prime} \ll n$, so the new embedding $X^{\prime}$ likely will not violate the constraints by much. It is also possible to require that the new embedding $X^{\prime}$ satisfy the constraints. This approach requires care; for example, suppose we are to add just one new item, i.e., $n^{\prime}=1$, and we insist that the new embedding $X^{\prime}$ be centered, like the original one 
$X$. In this case, the only possible choice for the vector associated with the new item is $x_{n+1}=0$.

Incremental embedding can be used to develop a feature map for new (unseen) items, given some distortion functions relating the new item to the original ones (say, the training items). In this case we have $n^{\prime}=1$; we embed the new item $n+1$ so as to minimize its average distortion with respect to the original items.

Placement problems. While MDE problems are usually nonconvex, anchored MDE problems with nondecreasing convex distortion functions are an exception. This means that some anchored MDE problems can be efficiently and globally solved. These MDE problems can be interpreted as (convex) placement problems, which are described in [BV04, §8.7] and have applications to VLSI circuit design [She12].

Semi-supervised learning. Anchored embeddings also arise in graph-based semisupervised learning problems. We interpret the vectors $x_{i}, i \in \mathcal{K}$, as the known labels; our job is to assign labels for $i \notin \mathcal{K}$, using a prior graph with positive weights that indicate similarity. In graph-based semi-supervised learning [Xu10; $\left.\mathrm{EA}^{+} 16\right]$, this is done by solving an anchored MDE problem with quadratic distortion functions $f_{i j}\left(d_{i j}\right)=w_{i j} d_{i j}^{2}$, which as mentioned above is convex and readily solved.

\subsubsection{Standardized embeddings}

The set of standardized embeddings is

$$
\mathcal{S}=\left\{X \mid(1 / n) X^{T} X=I, X^{T} \mathbf{1}=0\right\} .
$$

The standardization constraint $X \in \mathcal{S}$ requires that the collection of vectors $x_{1}, \ldots, x_{n}$ has zero mean and unit covariance. We can express this in terms of the $m$ feature columns of the embedding $X \in \mathcal{S}$ : they have zero mean, are uncorrelated, and have root-mean-square (RMS) value one; these properties are often desirable when the embedding is used in downstream machine learning tasks. We refer to the MDE problem as standardized when $\mathcal{X}=\mathcal{S}$.

Because $\mathcal{S}$ is a compact set, the standardized MDE problem has at least one solution. Since $\mathcal{S}$ is invariant under rotations, the standardized MDE problem has a family of solutions: if $X^{\star}$ is an optimal embedding, so is $X=X^{\star} Q$, for any $m \times m$ orthogonal matrix $Q$. 
Natural length in a standardized embedding. We note that for $X \in \mathcal{S}$, the sum of the squared embedding distances is constant,

$$
\sum_{1 \leq i<j \leq n} d_{i j}^{2}=n^{2} m
$$

(Note that this is the sum over all pairs $(i, j)$ with $i<j$, not just the pairs in $\mathcal{E}$.) To see this, we observe that

$$
\sum_{i, j=1}^{n} d_{i j}^{2}=2 n \sum_{i=1}^{n}\left\|x_{i}\right\|_{2}^{2}-2 \sum_{i, j=1}^{n} x_{i}^{T} x_{j} .
$$

The first term on the right-hand side equals $2 n^{2} m$, since

$$
\sum_{i=1}^{n}\left\|x_{i}\right\|_{2}^{2}=\operatorname{tr}\left(X X^{T}\right)=\operatorname{tr}\left(X^{T} X\right)=n m,
$$

while the second term equals 0 , since $X^{T} \mathbf{1}=0$. (Here $\operatorname{tr}$ denotes the trace of a matrix, i.e., the sum of its diagonal entries.)

From (2.6), we can calculate the RMS value of all $n(n-1) / 2$ embedding distances. We will refer to this value as the natural length $d_{\text {nat }}$ of the embedding, with

$$
d_{\mathrm{nat}}=\sqrt{\frac{2 n m}{n-1}} .
$$

For $X \in \mathcal{S}, d_{\text {nat }}$ can be interpreted as the typical value of embedding distances. We will see later that this number is useful in choosing appropriate distortion functions.

\subsubsection{Attraction, repulsion, and spreading}

When all distortion functions are nondecreasing, we say the objective is attractive. In the mechanical interpretation, this means that there is a positive (attractive) tension between all pairs of points $(i, j) \in \mathcal{E}$. Roughly speaking, the distortion functions only encode similarity between items, and not dissimilarity; the objective encourages neighboring embedded points to be near each other. When the only constraint is that the embedding is centered, i.e., $\mathcal{X}=\mathcal{C}$, a globally optimal embedding is $X=0$, which is not useful. Roughly speaking, all forces between points are attractive, so the points collapse to a single point, which must be 0 to achieve centering.

When the objective is attractive, we need a constraint to enforce spreading the points. Anchored and standardized constraints both serve this purpose, and enforce 
spreading of the points when the objective is attractive. When some distortion functions can be repulsive, i.e., $f_{i j}^{\prime}\left(d_{i j}\right)$ can be negative, we can encounter the opposite problem, which is that some points might spread without bound (i.e., the MDE problem does not have a solution). In such situations, an anchoring or standardized constraint can serve the role of keeping some points from spreading without bound.

The standardization constraint keeps both pathologies from happening. The points are required to be spread, and also bounded. Since $\mathcal{S}$ is compact, there is always a solution of the standardized MDE problem.

\subsubsection{Comparing embeddings}

In some cases we want to compare or evaluate a distance between two embeddings of the same set of items, $X$ and $\tilde{X}$. When the embeddings are anchored, a reasonable measure of how different they are is the mean-square distance between the two embeddings,

$$
\frac{1}{n}\|X-\tilde{X}\|_{F}^{2}=\frac{1}{n} \sum_{i=1}^{n}\left\|x_{i}-\tilde{x}_{i}\right\|_{2}^{2} .
$$

When the embeddings are centered or standardized, the comparison is more subtle, since in these cases any embedding can be transformed by an orthogonal matrix with no effect on the constraints or objective. As mentioned above, $\tilde{X}$ and $\tilde{X} Q$, where $Q^{T} Q=I$, are equivalent for the MDE problem. A reasonable measure of how different the two embeddings are is then

$$
\Delta=\inf \left\{\|X-\tilde{X} Q\|_{F}^{2} / n \mid Q^{T} Q=I\right\},
$$

the minimum mean-square distance over all orthogonal transformations of the second one (which is same as the minimum over transforming $X$, or both $X$ and $\tilde{X}$ ).

We can work out the distance $\Delta$ analytically, and also find an optimal $Q$, i.e., one that achieves the minimum. This is useful for visualization, if we are plotting $X$ or $\tilde{X}$ (presumably in the case with $m$ two or three): we plot both $X$ and $\tilde{X} Q$, the latter being the second embedding, optimally orthogonally transformed with respect to the first embedding. We refer to this as aligning the embedding $\tilde{X}$ to another target embedding $X$.

Finding the optimal $Q$ is an instance of the orthogonal Procrustes problem [Sch66]. We first form the $m \times m$ matrix $Z=X^{T} \tilde{X}$, and find its (full) singular value decomposition (SVD) $Z=U \Sigma V^{T}$. The optimal orthogonal matrix is $Q=V U^{T}$, which gives

$$
\Delta=\frac{1}{n}\left(\|X\|_{F}^{2}+\|\tilde{X}\|_{F}^{2}-2 \operatorname{tr} \Sigma\right) .
$$


For standardized embeddings we have $\|X\|_{F}^{2}=\|\tilde{X}\|_{F}^{2}=m n$, so $\Delta=m-\frac{2}{n} \operatorname{tr} \Sigma$.

\subsection{Simple examples}

We present some simple examples of synthetic MDE problems to illustrate some of the ideas discussed thus far. In each example, the original data is a graph on $n=20$ vertices, and we embed into $m=2$ dimensions. We will see more interesting examples, using real data, in part III of this monograph.

An unconstrained embedding. In our first example, the original graph has $p=$ 40 edges, which were randomly selected. In constructing the MDE problem data, we take $\mathcal{E}$ to be all $n(n-1) / 2$ pairs of the $n$ vertices, and assign deviations $\delta_{i j}$ between vertices $i$ and $j$ using the graph distance (i.e., the length of the shortest path between $i$ and $j$ ). We use quadratic distortion functions $f_{k}\left(d_{k}\right)=\left(d_{k}-\delta_{k}\right)^{2}$, and require $X \in \mathcal{C}$. The left plot in figure 2.5 shows a minimum-distortion embedding for this problem.

An anchored embedding. We embed the same original graph as the previous example, but in this example we impose an anchor constraint $X \in \mathcal{A}$. Seven of the vertices are anchors, and the rest are free. The anchors and their positions were selected randomly. The right plot in figure 2.5 shows a minimum-distortion embedding for this problem. The anchors are colored orange.

A standardized embedding. In the third example we produce standardized embeddings $X \in \mathcal{S}$ of a complete graph on 20 vertices. We compute two embeddings: the first is obtained by solving a standardized MDE problem with quadratic distortion functions $f_{k}\left(d_{k}\right)=d_{k}^{2}$, and the second uses cubic distortion functions $f_{k}\left(d_{k}\right)=d_{k}^{3}$. (These MDE problems can be interpreted as deriving from graphs, or from weights with $w_{i j}=1$ for all $\left.(i, j) \in \mathcal{E}\right)$.

The embeddings are plotted in figure 2.6. The embedding produced using quadratic distortion functions is known as a spectral layout, an old and widely used method for drawing graphs [Hal70; Kor03]. With cubic penalty, we obtain the embedding on the right, with the 20 points arranged uniformly spaced on a circle. This very simple example shows that the choice of distortion functions is important. With a quadratic distortion, most embedding distances are small but some are large; with a cubic distortion, which heavily penalizes large distances, no single embedding distance is very large. 

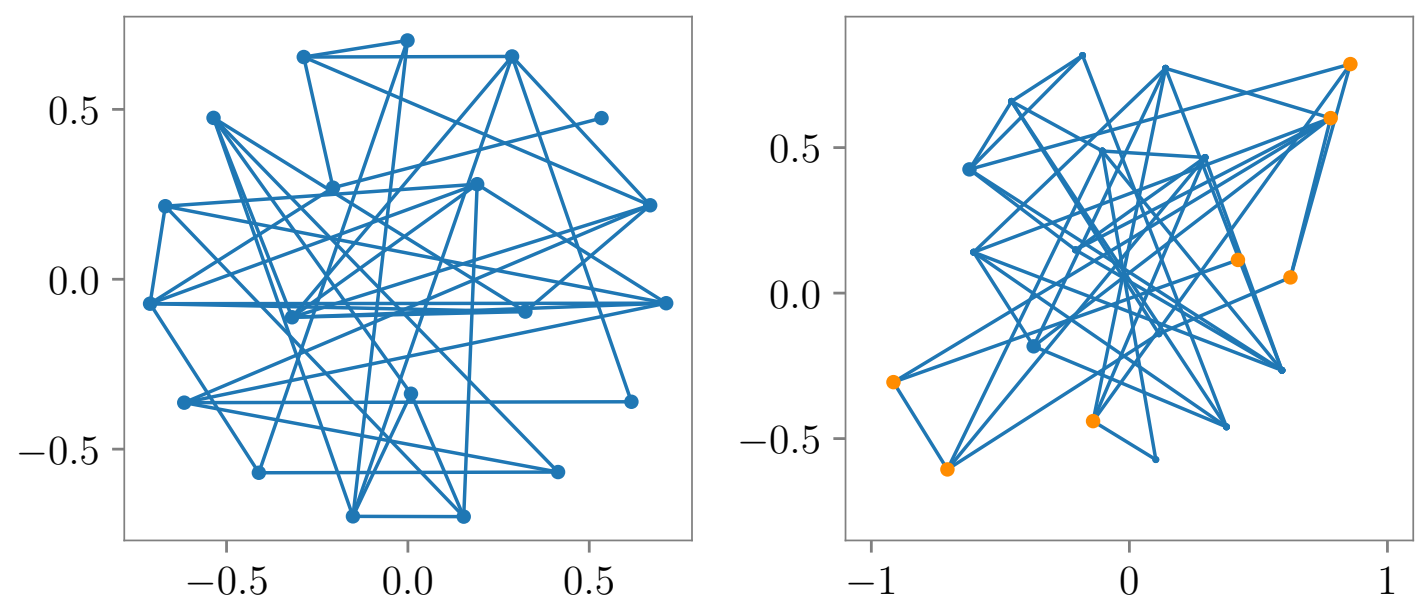

Figure 2.5: Embeddings derived from a graph on 20 vertices and 40 edges. Left. Unconstrained. Right. Anchored (orange points are anchors).
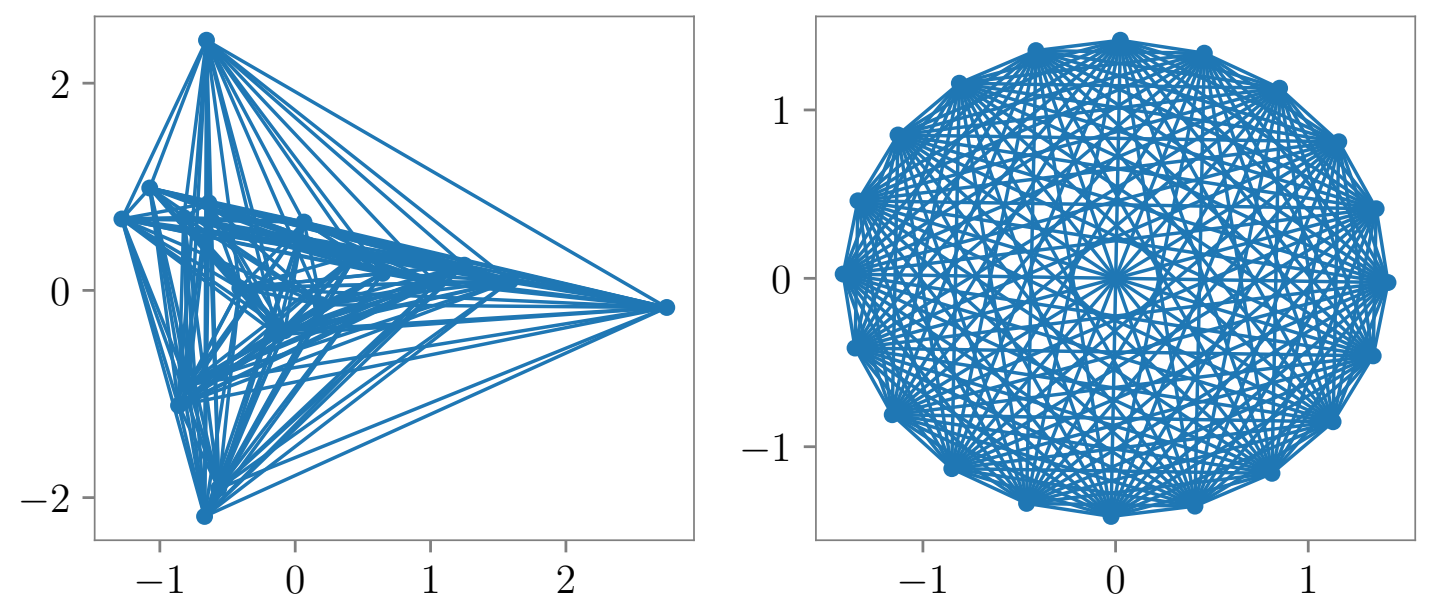

Figure 2.6: Standardized embeddings on a complete graph. Left. Quadratic penalty. Right Cubic penalty. 


\subsection{Validation}

The quality of an embedding ultimately depends on whether it is useful for the downstream application of interest. Nonetheless, there are simple methods that can be used to sanity check an embedding, independent of the downstream task. We describe some of these methods below. (We will use some of these methods to sanity check embeddings involving real data in part III.)

Using held-out attributes. In the original data, some or all of the items may be tagged with attributes. For example, if the items are images of handwritten digits, some of the images may be tagged with the depicted digit; if the items are singlecell mRNA transcriptomes collected from several donors, the transcriptomes may be tagged with their donors of origin; or if the items are researchers, they may be tagged with their field of study.

If an attribute is held-out, i.e., if the MDE problem does not depend on it, it can be used to check whether the embedding makes sense. In particular, we can check whether items with the same attribute value are near each other in the embedding. When the embedding dimension is 1,2 or 3, this can be done visually by arranging the embedding vectors in a scatter plot, and coloring each point by its attribute value. When the dimension is greater than 3 , we can check if a model can be trained to predict the attribute, given the embedding vectors. If items tagged with the same or similar values appear near each other in the embedding, we can take this a weak endorsement of the embedding. Of course this requires that the held-out attributes are related to whatever attributes were used to create the MDE problem.

Examining high-distortion pairs. Thus far we have only been concerned with the average distortion of an embedding. It can also be instructive to examine the distribution of distortions associated with an embedding.

Suppose most pairs $(i, j) \in \mathcal{E}$ have low distortion, but some have much higher distortion. This suggests that the high-distortion pairs may contain anomalous items, or that the pairing itself may be anomalous (e.g., dissimilar items may have been designated as similar). If we suspect that these outlier pairs have an outsize effect on the embedding (e.g., if their sum of distortions is a sizable fraction of the total distortion), we might modify the distortion functions to be more robust, i.e., to not grow as rapidly for large distances, or simply throw out these outliers and re-embed.

Checking for redundancy. A third method is based on the vague idea that a good embedding should not be too sensitive to the construction of the MDE problem. In 
particular, it should not require all the distortion functions.

We can check whether there is some redundancy among the distortion functions by holding out a subset of them, constructing an embedding without these functions, and then evaluating the average distortion on the held-out functions. We partition $\mathcal{E}$ into training and held-out sets,

$$
\mathcal{E}_{\text {train }} \subseteq \mathcal{E}, \quad \mathcal{E}_{\text {val }}=\mathcal{E} \backslash \mathcal{E}_{\text {train }},
$$

and find an embedding $X$ using only the pairs in the training set $\mathcal{E}_{\text {train }}$. We then compare the average distortions on the training set and the validation set,

$$
f_{\text {train }}=\frac{1}{\left|\mathcal{E}_{\text {train }}\right|} \sum_{(i, j) \in \mathcal{E}_{\text {train }}} f_{i j}\left(d_{i j}\right), \quad f_{\text {val }}=\frac{1}{\left|\mathcal{E}_{\text {val }}\right|} \sum_{(i, j) \in \mathcal{E}_{\text {val }}} f_{i j}\left(d_{i j}\right) .
$$

While we would expect that $f_{\text {val }} \geq f_{\text {train }}$, if $f_{\text {val }}$ is much larger than $f_{\text {train }}$, it might suggest that we do not have enough edges (and that we should obtain more).

Checking for bias. In many applications, it is important to ensure various groups of items are treated (in some sense) fairly and equitably by the embedding (see, e.g., $\left[\mathrm{Dwo}^{+} 12 ; \mathrm{Bol}^{+} 16\right.$; BHN18; CDG18; $\left.\mathrm{Gar}^{+} 18 ; \mathrm{Hol}^{+} 19\right]$ ). For example, consider the task of embedding English words. It has been shown that some popular embedding methods reinforce harmful stereotypes related to gender, race, and mental health; e.g., in the BERT language model $\left[\mathrm{Dev}^{+} 19\right]$, words related to disabilities have been found to be associated with negative sentiment words [Hut $\left.{ }^{+} 20\right]$. Moreover, for embedding methods (like BERT) that are trained to model an extremely large corpus of natural language documents, it is usually difficult to correct these biases, even if they have been identified in the embedding; Bender, Gebru, McMillan-Major, et al. $\left[\mathrm{Ben}^{+} 21\right]$ call this the problem of unfathomable training data.

The MDE framework provides a principled way of detecting and mitigating harmful bias in an embedding. In our framework, there are at least two ways in which harmful biases may manifest: the distortion functions may encode harmful biases, or the embedding may have lower distortion on the edges associated with one group than another. Along these lines, we propose the following three-step procedure to mitigate bias. We continue with our running example of embedding English words, but the procedure applies more broadly.

1. Identify groups of interest. The first step is to identify groups of interest. This might correspond to identifying sets of words related to gender, sex, race, disabilities, and other demographic categories. (These sets need not be disjoint.) 
2. Audit the distortion functions. The second step is to audit to distortion functions in which at least one item is a member of an identified group, and modifying them if they encode harmful biases.

For example, say the distortion functions are derived from weights, and the modeler is interested in words related to the female sex. The modeler should audit the functions $f_{i j}$ for which $i$ or $j$ is related to the female sex. In particular, the modeler may want to ensure that the weights associating these words to certain professions and adjectives are small, zero, or negative. The modeler can also add or modify distortion functions between words in different groups, to articulate whether, e.g., the vectors for "man" and "woman" should be close or not close in the embedding.

This step is extremely important, since the embedding is designed to be faithful to the relationships articulated by the distortion functions. It is also difficult, because it requires that the modeler be conscious of the many kinds of harmful biases that may arise in the embedding.

3. Compare distortions. The third step is to compute the embedding, and check whether it is more faithful to the stated relationships involving one group than another. This can be done by comparing the distortions for different groups.

For example, say we have two groups of interest: $G_{1} \subseteq \mathcal{V}$ and $G_{2} \subseteq \mathcal{V}$. Let $\mathcal{E}_{1}$ be the set of pairs $(i, j)$ in which $i \in G_{1}$ or $j \in G_{1}$, and let $\mathcal{E}_{2}$ be the same for $G_{2}$. We can compute the average distortion of the embedding restricted to these two sets:

$$
E_{1}(X)=\frac{1}{\left|\mathcal{E}_{1}\right|} \sum_{(i, j) \in \mathcal{E}_{1}} f_{i j}\left(d_{i j}\right), \quad E_{2}(X)=\frac{1}{\left|\mathcal{E}_{2}\right|} \sum_{(i, j) \in \mathcal{E}_{2}} f_{i j}\left(d_{i j}\right) .
$$

Suppose $E_{1}(X) \approx E_{2}(X)$. This means that on average, the embedding is as faithful to the stated relationships involving group $G_{1}$ as it is to the relationships involving group $G_{2}$. (Importantly, $E_{1}(X) \approx E_{2}(X)$ does not mean that the embedding is free of harmful bias, since the distortion functions themselves may encode harmful bias if the modeler did a poor job in step 2.)

Otherwise, if $E_{1}(X)<E_{2}(X)$, the embedding is on average more faithful to the first group than the second, revealing a type of bias. The modeler can return to step 2 and modify the distortion functions so that $\mathcal{E}_{2}$ contributes more to the total distortion (e.g., by increasing some of the the weights associated with $\mathcal{E}_{2}$, or decreasing some of the weights associated with $\mathcal{E}_{1}$ ). 
We emphasize that ensuring an embedding is fair and free of harmful bias is very difficult, since it requires the modelers to be aware of the relevant groups and biases. All the MDE framework does is provide modelers a procedure that can help identify and mitigate harmful biases, provided they are aware of them. 


\section{Chapter 3}

\section{Quadratic MDE Problems}

In this chapter we consider MDE problems with quadratic distortion functions,

$$
f_{i j}\left(d_{i j}\right)=w_{i j} d_{i j}^{2}, \quad(i, j) \in \mathcal{E}
$$

where $w_{i j}$ is a weight encoding similarities between items. Large positive $w_{i j}$ means items $i$ and $j$ are very similar, small or zero $w_{i j}$ means they are neither similar nor dissimilar, and large negative $w_{i j}$ means the two items are very dissimilar. In many cases the weights are all nonnegative, in which case the objective is attractive. We refer to an MDE problem with quadratic distortion functions as a quadratic $M D E$ problem.

Centered embeddings. Centered (unconstrained) quadratic MDE problems are not interesting. In all cases, either $X=0$ is a global solution, or the problem is unbounded below, so there is no solution.

Anchored embeddings. Anchored quadratic MDE problems with positive weights are readily solved exactly by least squares. This problem is sometimes called the quadratic placement problem [SDJ91], and has applications in circuit design. When there are negative weights, the anchored quadratic MDE problem can be unbounded below, i.e., not have a solution.

Standardized embeddings. For the remainder of this chapter we focus on the standardized quadratic MDE problem,

$$
\begin{array}{ll}
\operatorname{minimize}_{\text {subject to }} & (1 / p) \sum_{(i, j) \in \mathcal{E}} w_{i j} d_{i j}^{2} \\
\text { sub. }
\end{array}
$$


These problems are also sometimes referred to as quadratic placement problems, e.g., as in [Hal70].

Standardized quadratic MDE problems are special for several reasons. First, they are tractable: we can solve (3.1) globally, via eigenvector decomposition, as we will see in §3.1. Second, many well-known historical embeddings can be obtained by solving instances of the quadratic MDE problem, differing only in their choice of weights, as shown in $\S 3.2$.

\subsection{Solution by eigenvector decomposition}

We can re-formulate the problem (3.1) as an eigenproblem. Note that

$$
E(X)=1 / p \sum_{(i, j) \in \mathcal{E}} w_{i j} d_{i j}^{2}=1 / p \operatorname{tr}\left(X^{T} L X\right),
$$

where the symmetric matrix $L \in \mathbf{S}^{n}$ has upper triangular entries $(i . e ., i<j$ ) given by

$$
L_{i j}= \begin{cases}-w_{i j} & (i, j) \in \mathcal{E} \\ 0 & \text { otherwise }\end{cases}
$$

and diagonal entries

$$
L_{i i}=-\sum_{j \neq i} L_{i j} .
$$

(The lower triangular entries are found from $L_{i j}=L_{j i}$.) We note that $L$ satisfies $L \mathbf{1}=0$. If the weights are all nonnegative, the matrix $L$ is a Laplacian matrix. But we do not assume here that all the weights are nonnegative.

The equality (3.2) follows from the calculation

$$
\begin{aligned}
1 / p \sum_{(i, j) \in \mathcal{E}} w_{i j} d_{i j}^{2} & =1 / p \sum_{(i, j) \in \mathcal{E}} w_{i j}\left\|x_{i}-x_{j}\right\|_{2}^{2} \\
& =1 / p \sum_{(i, j) \in \mathcal{E}} w_{i j}\left(\left\|x_{i}\right\|_{2}^{2}+\left\|x_{j}\right\|_{2}^{2}-2 x_{i}^{T} x_{j}\right) \\
& =1 / p\left(\sum_{i=1}^{n} L_{i i}\left\|x_{i}\right\|_{2}^{2}+2 \sum_{(i, j) \in \mathcal{E}} L_{i j} x_{i}^{T} x_{j}\right) \\
& =1 / p \operatorname{tr}\left(X^{T} L X\right) .
\end{aligned}
$$


The MDE problem (3.1) is therefore equivalent to the problem

$$
\begin{array}{ll}
\text { minimize } & \operatorname{tr}\left(X^{T} L X\right) \\
\text { subject to } & X \in \mathcal{S},
\end{array}
$$

a solution to which can be obtained from eigenvectors of $L$. To see this, we first take an eigenvector decomposition of $L$, letting $v_{1}, v_{2}, \ldots, v_{n}$ be orthonormal eigenvectors,

$$
L v_{i}=\lambda_{i} v_{i}, \quad i=1, \ldots, n, \quad \lambda_{1} \leq \cdots \leq \lambda_{n} .
$$

Let $\tilde{x}_{1}, \tilde{x}_{2}, \ldots, \tilde{x}_{m} \in \mathbf{R}^{n}$ be the columns of $X$ (which we recall satisfies $X^{T} X=n I$ ). Then

$$
\begin{aligned}
(1 / p) \operatorname{tr}\left(X^{T} L X\right) & =(1 / p) \sum_{i=1}^{n} \lambda_{i}\left(\left(v_{i}^{T} \tilde{x}_{1}\right)^{2}+\left(v_{i}^{T} \tilde{x}_{2}\right)^{2}+\cdots+\left(v_{i}^{T} \tilde{x}_{m}\right)^{2}\right) \\
& \geq(n / p) \sum_{i=1}^{m} \lambda_{i}
\end{aligned}
$$

where the inequality follows from

$$
\left(v_{i}^{T} \tilde{x}_{1}\right)^{2}+\left(v_{i}^{T} \tilde{x}_{2}\right)^{2}+\cdots+\left(v_{i}^{T} \tilde{x}_{m}\right)^{2} \leq n
$$

for $i=1, \ldots, n$, and

$$
\sum_{i=1}^{n} \sum_{j=1}^{m}\left(v_{i}^{T} \tilde{x}_{j}\right)^{2}=n m .
$$

Let $v_{j}$ be the eigenvector that is a multiple of 1 . If $j>m$, a minimum-distortion embedding is given by $\tilde{x}_{i}=\sqrt{n} v_{i}$, i.e., by concatenating the bottom $m$ eigenvectors,

$$
X=\sqrt{n}\left[v_{1} \cdots v_{m}\right] .
$$

If $j \leq m$, a solution is obtained by removing $v_{j}$ from the above and appending $v_{m+1}$,

$$
X=\sqrt{n}\left[v_{1} \cdots v_{j-1} v_{j+1} \cdots v_{m+1}\right] .
$$

The optimal value of the MDE problem is

$$
\frac{n}{p}\left(\lambda_{1}+\cdots+\lambda_{m}\right)
$$

for $j>m$, or

$$
\frac{n}{p}\left(\lambda_{1}+\cdots+\lambda_{m+1}\right)
$$

for $j \leq m$. 
Computing a solution. To compute $X$, we need to compute the bottom $m+1$ eigenvectors of $L$. For $n$ not too big (say, no more than 10,000), it is possible to carry out a full eigenvector decomposition of $L$, which requires $O\left(n^{2}\right)$ storage and $O\left(n^{3}\right)$ flops. This naïve method is evidently inefficient, since we will only use $m+1$ of the $n$ eigenvectors, and while storing $X$ requires only $O(n m)$ storage, this method requires $O\left(n^{2}\right)$. For $n$ larger than around 10,000, the cubic complexity becomes prohibitively expensive. Nonetheless, when $L$ is dense, which happens when $p$ is on the order of $n^{2}$, we cannot do much better than computing a full eigenvector decomposition.

For sparse $L\left(p \ll n^{2}\right)$ we can use iterative methods that only require multiplication by $L$, taking $O(p)$ flops per iteration; these methods compute just the bottom $m$ eigenvectors that we seek. One such method is the Lanczos iteration [Lan51], an algorithm for finding eigenvectors corresponding to extremal eigenvalues of symmetric matrices. Depending on the spectrum of $L$, the Lanczos method typically reaches a suitable solution within $O(n)$ or sometimes even $O(1)$ iterations [TB97, $\S 32]$. Another popular iterative method is the locally optimal preconditioned conjugate gradient method (LOBPCG), which also applies to symmetric matrices [Kny01]. Lanczos iteration and LOBPCG belong to a family of methods involving projections onto Krylov subspaces; for details, see [TB97, §32, §36] and [GVL13, §10].

\subsection{Historical examples}

In this section we show that many existing embedding methods reduce to solving a quadratic MDE problem (3.1), with different methods using different weights.

Laplacian embedding. When the weights are all nonnegative, the matrix $L$ is a Laplacian matrix for the graph $(\mathcal{V}, \mathcal{E})$ (with edge weights $w_{i j}$ ). The bottom eigenvector $v_{1}$ of $L$ is a multiple of $\mathbf{1}$, so a solution is $X=\left[v_{2} \cdots v_{m+1}\right]$; an embedding obtained in this way is known as a Laplacian embedding or spectral embedding. Laplacian embedding is the key computation in spectral clustering, a technique for clustering the nodes of a graph [PSL90; vL07]. (We mention that spectral clustering can be extended to graphs with negative weights [ $\mathrm{Kun}^{+} 10$; Kny17; Kny18]). In machine learning, Laplacian embedding is a popular tool for dimensionality reduction, known as the Laplacian eigenmap [BN02].

A variant of the Laplacian embedding uses the bottom eigenvectors of the normalized Laplacian matrix, $L_{\text {norm }}=D^{-1 / 2} L D^{-1 / 2}$, where $D \in \mathbf{R}^{n \times n}$ is a diagonal matrix with entries $D_{i i}=L_{i i}$. This variant can be expressed as a quadratic MDE problem via the change of variables $Y=D^{-1 / 2} X$. 
Principal component analysis. PCA starts with data $y_{1}, \ldots, y_{n} \in \mathbf{R}^{q}$, assumed to be centered (and with $q \geq m$ ). The data are assembled into a matrix $Y \in \mathbf{R}^{n \times q}$ with rows $y_{i}^{T}$. The choice of weights

$$
w_{i j}=y_{i}^{T} y_{j}
$$

for $(i, j) \in \mathcal{E}=\{(i, j) \mid 1 \leq i<j \leq n\}$ yields an MDE problem that is equivalent to $\mathrm{PCA}$, in the following sense. The matrix $L$ corresponding to this choice of weights has entries

Because the data is centered,

$$
L_{i j}= \begin{cases}-y_{i}^{T} y_{j} & i \neq j \\ \sum_{j: i \neq j} y_{i}^{T} y_{j} & i=j .\end{cases}
$$

$$
\sum_{j: i \neq j} y_{i}^{T} y_{j}=-y_{i}^{T} y_{i}, \quad i=1, \ldots, n,
$$

so the diagonal of $L$ has entries $-y_{1}^{T} y_{1}, \ldots,-y_{n}^{T} y_{n}$. In particular, $L=-Y Y^{T}$. Hence, the low-dimensional embedding of $Y$ obtained by PCA, which takes $X$ to be the $m$ top eigenvectors of $Y Y^{T}$ [Ude $\left.{ }^{+} 16, \S 2\right]$, is also a solution of the MDE problem.

The MDE formulation of PCA has a natural interpretation, based on the angles between the data vectors. When the angle between $y_{i}$ and $y_{j}$ is acute, the weight $w_{i j}$ is positive, so items $i$ and $j$ are considered similar. When the angle is obtuse, the weight $w_{i j}$ is negative, so items $i$ and $j$ are considered dissimilar. When the data vectors are orthogonal, $w_{i j}$ is zero, so the embedding is neutral on the pair $i, j$. If the data vectors each have zero mean, there is an additional interpretation: in this case, PCA seeks to place vectors associated with positively correlated pairs near each other, anti-correlated pairs far from each other, and is neutral on uncorrelated pairs.

Kernel PCA. Like PCA, kernel PCA starts with vectors $y_{1}, \ldots, y_{n}$, but it replaces these vectors by nonlinear transformations $\phi\left(y_{1}\right), \ldots, \phi\left(y_{n}\right)$. We define the kernel matrix $K \in \mathbf{S}^{n}$ of $\phi\left(y_{1}\right), \ldots, \phi\left(y_{n}\right)$ as

$$
K_{i j}=\phi\left(y_{i}\right)^{T} \phi\left(y_{j}\right) .
$$

Kernel PCA applies PCA to the matrix $K^{1 / 2}\left(I-\mathbf{1 1}^{T} / n\right)$, i.e., it computes the bottom eigenvectors of the matrix

$$
L=-\left(I-\mathbf{1 1}^{T} / n\right) K\left(I-\mathbf{1 1}^{T} / n\right) .
$$

By construction, $L$ is symmetric, and its rows sum to $0(i . e ., L \mathbf{1}=0)$; therefore, the choice of weights

$$
w_{i j}=-L_{i j}, \quad 1 \leq i<j \leq n,
$$

yields an MDE problem equivalent to kernel PCA. 
Locally linear embedding. Locally linear embedding (LLE) seeks an embedding so that each item is approximately reconstructed by a linear combination of the embedding vectors associated with its nearest neighbors [RS00; SR01]. As in PCA, the data is a list of vectors $y_{1}, \ldots, y_{n}$. A linearly-constrained least squares problem is solved to find a matrix $W \in \mathbf{S}^{n}$ that minimizes the reconstruction error

$$
\sum_{i=1}^{n}\left\|y_{i}-\sum_{j=1}^{n} W_{i j} y_{j}\right\|_{2}^{2},
$$

subject to the constraints that the rows of $W$ sum to 1 and $W_{i j}=0$ if $y_{j}$ is not among the $k$-nearest neighbors of $y_{i}$, judged by the Euclidean distance ( $k$ is a parameter). LLE then obtains an embedding by computing the bottom eigenvectors of

$$
L=(I-W)^{T}(I-W) .
$$

Once again, $L$ is symmetric with rows summing to 0 , so taking $w_{i j}=-L_{i j}$ results in an equivalent MDE problem.

Classical multidimensional scaling. Classical multidimensional scaling (MDS) is an algorithm for embedding items, given original distances or deviations $\delta_{i j}$ between all $n(n-1) / 2$ item pairs [Tor52]. The original distances $\delta_{i j}$ are arranged into a matrix $D \in \mathbf{S}^{n}$, with

$$
D_{i j}=\delta_{i j}^{2} .
$$

Classical MDS produces an embedding by computing the bottom eigenvectors of

$$
L=\left(I-\mathbf{1 1}^{T} / n\right) D\left(I-\mathbf{1 1}^{T} / n\right) / 2 .
$$

This matrix is symmetric, and its rows sum to 0 . Therefore, choosing

$$
w_{i j}=-L_{i j}, \quad 1 \leq i<j \leq n,
$$

results in an MDE equivalent to classical MDS.

When the original distances are Euclidean, the weights $w_{i j}$ are the inner products between the (centered) points that generated the original distances; if these points were in fact vectors in $\mathbf{R}^{m}$, then classical MDS will recover them up to rotation. But when the original distances are not Euclidean, it is not clear what $w_{i j}$ represents, or how the optimal embedding distances relate to the original distances. In $\S 4.2$, we show how distortion functions can be chosen to preserve original distances directly. 
Isomap. Isomap is a well-known dimensionality reduction method that reduces to classical MDS [TDSL00; $\mathrm{Ber}^{+} 00$ ] and therefore is also an MDE of the form (3.1). Like classical MDS, Isomap starts with original distances $\delta_{i j}$ for all pairs of items. Isomap then constructs a shortest path metric on the items, and runs classical MDS on that metric. Specifically, it constructs a graph with $n$ nodes, in which an edge exists between $i$ and $j$ if $\delta_{i j}<\epsilon$, where $\epsilon>0$ is a parameter. If an edge between $i$ and $j$ exists it is weighted by $\delta_{i j}$, and the shortest path metric is constructed from the weighted graph in the obvious way.

Maximum variance unfolding. Maximum variance unfolding is another dimensionality reduction method that starts with an original data matrix and reduces to PCA [WS04]. For each item $i$, maximum variance unfolding computes its $k$-nearest neighbors under the Euclidean distance, where $k$ is a parameter, obtaining $n k$ original distances $\delta_{i j}$. Next, the method computes a (centered) Gram matrix $G$ of maximum variance that is consistent with the $\delta_{i j}$, i.e., such that $G_{i i}-2 G_{i j}+G_{j j}=\delta_{i j}^{2}$ for each $\delta_{i j}$; this matrix can be found by solving a semidefinite program [WS04, §3.2]. Finally, like PCA, maximum variance unfolding takes the $m$ top eigenvectors of $G$ (or the $m$ bottom eigenvectors of $L=-G$ ) as the embedding. (When solving the semidefinite program is difficult, maximum variance unfolding can be approximated by a method called maximum variance correction [CWC13].) 


\section{Chapter 4}

\section{Distortion Functions}

In this chapter we give examples of distortion functions. We organize these into those that derive from given weights, and those that derive from given original distances or deviations between items, although the distinction is not sharp.

\subsection{Functions involving weights}

In many MDE problems, the similarities between items are described by nonzero weights $w_{i j}$ for $(i, j) \in \mathcal{E}$, with positive weights indicating similar items and negative weights indicating dissimilar items. In this setting, the edges are partitioned into two sets: $\mathcal{E}_{\text {sim }}$ (positive weights, i.e., similar items) and $\mathcal{E}_{\text {dis }}$ (negative weights, dissimilar items).

We mention some special cases. In the simplest case, all weights are equal to one. This means that the original data simply tells us which pairs of items are similar. Another common situation is when all weights are positive, which specifies varying levels of similarity among the items, but does not specify dissimilarity. Another common case is when the weights have only two values, +1 and -1 . In this case we are specifying a set of pairs of similar items, and a (different) set of pairs of dissimilar items. In the most general case, the weights can be positive or negative, indicating similar and dissimilar pairs of items, and different degrees of similarity and dissimilarity.

The weights are given as, or derive from, the raw data of an application. As an example consider a social network, with $(i, j) \in \mathcal{E}$ meaning individuals $i$ and $j$ are acquainted. A reasonable choice for the weight $w_{i j}$ might be one plus the number of acquaintances individuals $i$ and $j$ have in common. (This provides an example of preprocessing, i.e., deriving weights from the original data, which in this exam- 
ple is the unweighted acquaintance graph. We describe several other preprocessing methods in §8.2.)

Distortion functions. For problems involving weights, distortion functions have the form

$$
f_{i j}\left(d_{i j}\right)=\left\{\begin{array}{ll}
w_{i j} p_{\mathrm{s}}\left(d_{i j}\right) & (i, j) \in \mathcal{E}_{\mathrm{sim}} \\
w_{i j} p_{\mathrm{d}}\left(d_{i j}\right) & (i, j) \in \mathcal{E}_{\mathrm{dis}}
\end{array}, \quad(i, j) \in \mathcal{E},\right.
$$

where $p_{\mathrm{s}}: \mathbf{R}_{+} \rightarrow \mathbf{R}$ and $p_{\mathrm{d}}: \mathbf{R}_{+} \rightarrow \mathbf{R}$ are increasing penalty functions. Thus for $(i, j) \in \mathcal{E}_{\text {sim }}\left(w_{i j} \geq 0\right)$, the distortion function $f_{i j}$ is attractive (increasing). For $(i, j) \in \mathcal{E}_{\text {dis }}\left(w_{i j}<0\right), f_{i j}$ is repulsive (decreasing). When $\mathcal{E}_{\text {dis }}$ is empty, the weights are all positive and the objective $E$ is attractive. In this case the unconstrained problem has the trivial solution $X=0$; we can use an anchor or standardization constraint to enforce spreading and avoid this pathology.

We have found that the choice of a penalty function matters much more than the choice of weights (in many applications, using +1 and -1 weights suffices). While there are many possible penalty functions, in practice they can be characterized by how they treat small distances, and how they treat large distances. These two vague qualities largely determine the characteristics of the embedding. For this reason, it suffices to use just a few simple penalty functions (out of the many possible choices). In the next two subsections, we describe some attractive and repulsive penalty functions that we have found to be useful.

\subsubsection{Attractive distortion functions}

Here we give a few examples of functions that work well as attractive penalties. We start with powers, the simplest family of penalty functions. This family is parametrized by the exponent, which determines the relative contributions of small and large distances to the distortion. Then we describe three penalties that have gentle slopes for small distances, and larger (but not large) slopes for large distances; these typically result in embeddings in which different "classes" of items are wellseparated, but items within a single class are not too close.

Powers. The simplest family of penalty functions is the power penalty,

$$
p_{\mathrm{s}}(d)=d^{\alpha},
$$

where $\alpha>0$ is a parameter. The larger $\alpha$ is, the more heavily large distances are penalized, and the less heavily small distances are penalized. This is illustrated in 


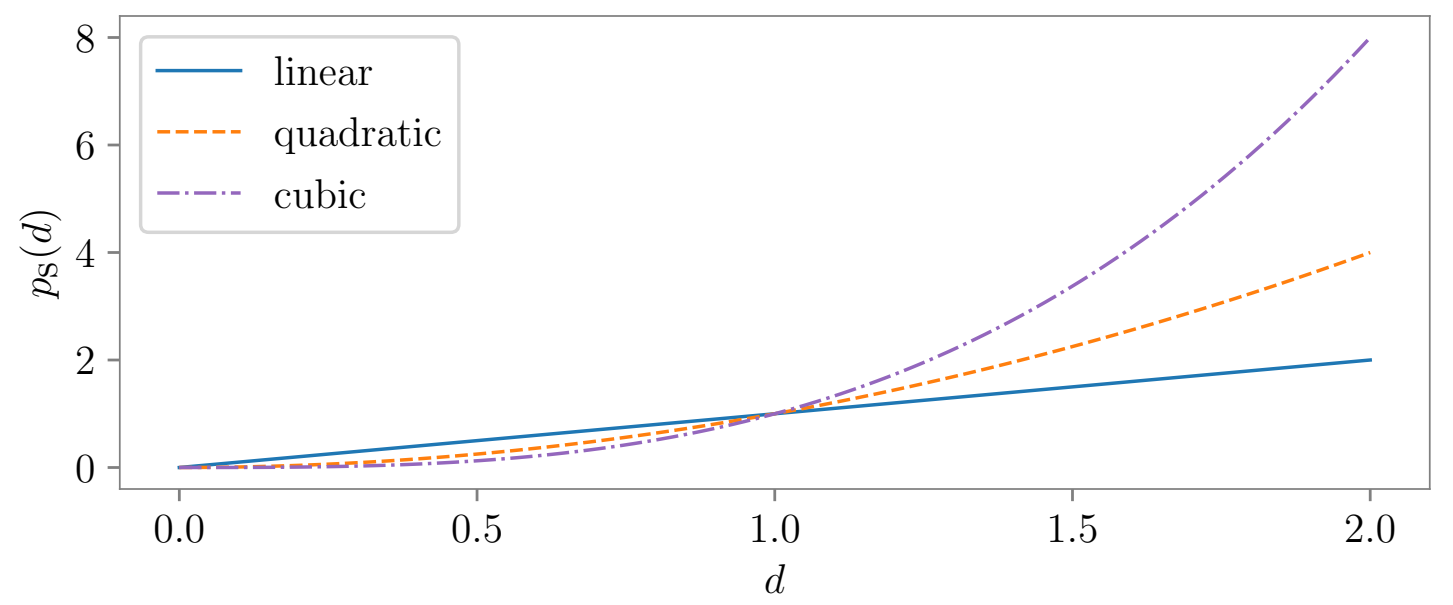

Figure 4.1: Powers. A linear, quadratic, and cubic penalty.

figure 4.1, which plots linear $(\alpha=1)$, quadratic $(\alpha=2)$, and cubic $(\alpha=3)$ penalties. For $x>1$, the cubic penalty is larger than than the quadratic, which is in turn larger than the linear penalty. For $x<1$, the opposite is true.

Decreasing $\alpha$ increases the penalty for small distances and decreases the slope for large distances. So smaller values of $\alpha$ causes similar items to more closely cluster together in the embedding, while allowing for some embedding distances to be large. In contrast when $\alpha$ is large, the slope of the penalty for small distances is small and the slope for large distances is large; this results in embeddings in which the points are "spread out" so that no one embedding distance is too large.

Huber penalty. The Huber penalty is

$$
p_{\mathbf{s}}(d)= \begin{cases}d^{2} & d<\tau \\ \tau(2 d-\tau) & d \geq \tau,\end{cases}
$$

where $\tau$ (the threshold) is a positive parameter. This penalty function is quadratic for $d<\tau$ and affine for $d>\tau$. Because the penalty is quadratic for small distances, the embedding vectors do not cluster too closely together; because it is linear for large distances, some embedding distances may be large, though most will be small.

Logistic penalty. The logistic penalty has the form

$$
p_{\mathrm{s}}(d)=\log \left(1+e^{\alpha(d-\tau)}\right),
$$




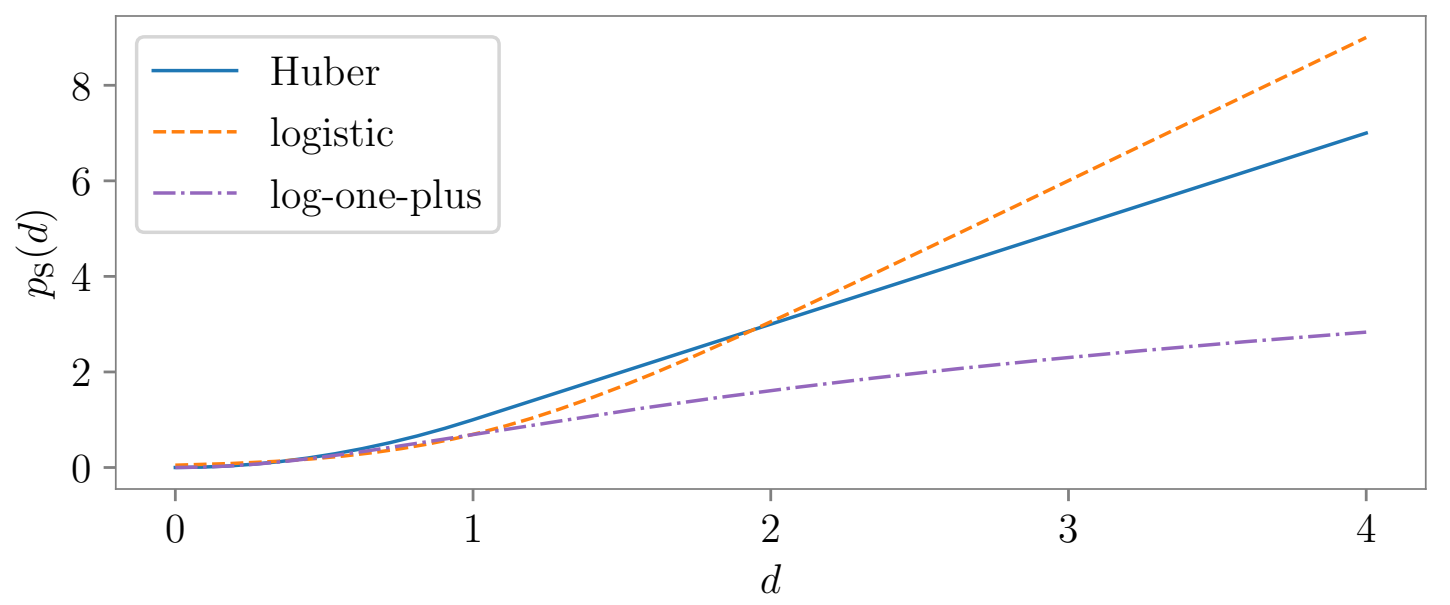

Figure 4.2: More penalties. A Huber, logistic, and log-one-plus penalty.

where $\alpha>0$ and $\tau>0$ are parameters. The logistic penalty encourages similar items to be smaller than the threshold $\tau$, while charging a roughly linear cost to distances larger than $\tau$.

Log-one-plus penalty. The log-one-plus penalty is

$$
p_{\mathrm{s}}(d)=\log \left(1+d^{\alpha}\right),
$$

where $\alpha>0$ is a parameter. For small $d$, the log-one-plus penalty is close to $d^{\alpha}$, a power penalty with exponent $\alpha$, but for large $d, \log \left(1+d^{\alpha}\right) \approx \alpha \log d$, which is much smaller than $d^{\alpha}$.

The dimensionality reduction methods t-SNE [MH08], LargeVis [ $\left.\mathrm{Tan}^{+} 16\right]$, and UMAP [MHM18] use this function (or a simple variant of it) as an attractive penalty.

\subsubsection{Repulsive distortion functions}

Repulsive distortion functions are used to discourage vectors associated with dissimilar items from being close. Useful repulsive penalties are barrier functions, with $p_{\mathrm{d}}(d)$ converging to $-\infty$ as $d \rightarrow 0$, and converging to 0 as $d \rightarrow \infty$. This means that the distortion, which has the form $w_{i j} p_{\mathrm{d}}\left(d_{i j}\right)$ with $w_{i j}<0$, grows to $\infty$ as the distance $d_{i j}$ approaches zero, and converges to zero as $d_{i j}$ becomes large.

We give two examples of such penalties below, and plot them in figure 4.3. 


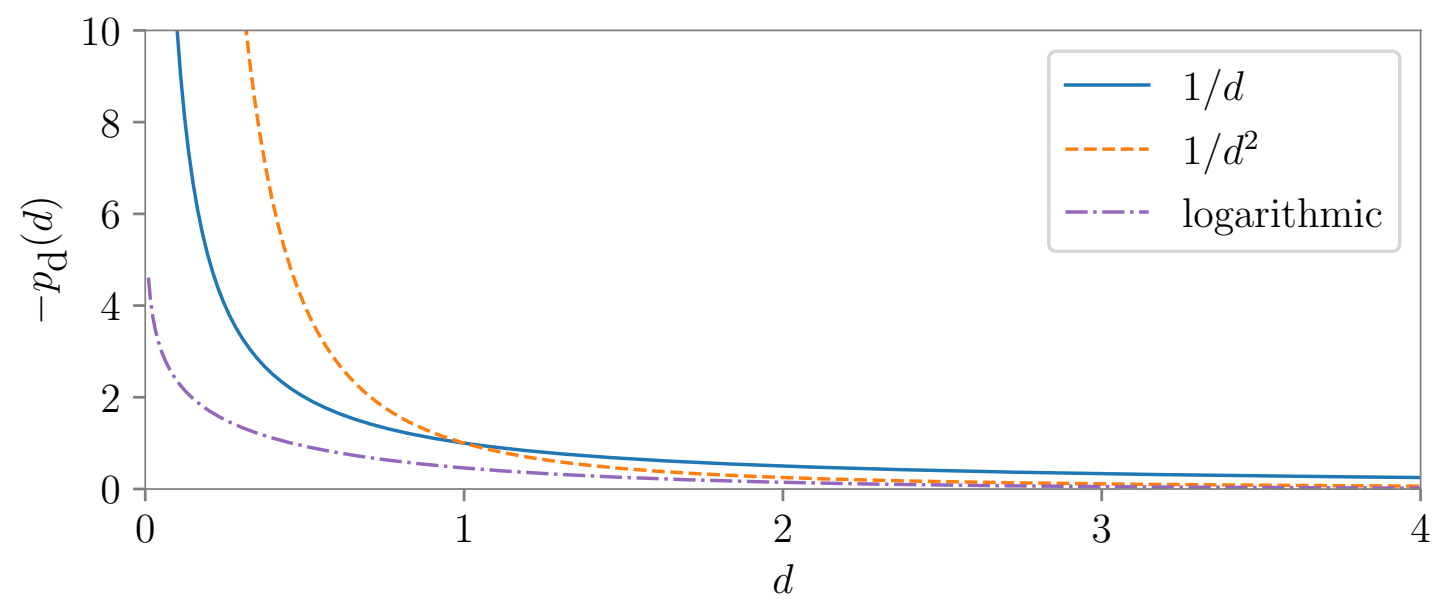

Figure 4.3: Repulsive penalties. Inverse power and logarithmic penalties.

Inverse power penalty. The inverse power penalty is

$$
p_{\mathrm{d}}(d)=-1 / d^{\alpha}
$$

where $\alpha>0$ is a parameter. When used in unconstrained MDE problems, this penalty sometimes results in a few embedding vectors being very far from the others. This pathology can be avoided by imposing a standardization constraint.

Logarithmic penalty. The logarithmic penalty is

$$
p_{\mathrm{d}}(d)=\log \left(1-\exp \left(-d^{\alpha}\right)\right)
$$

where $\alpha>0$ is a parameter. We have found that the logarithmic penalty is effective with or without the standardization constraint. We will see some examples of embeddings produced using logarithmic penalties in part III of this monograph.

Many other repulsive penalties can and have been used, including for example the barrier

$$
p_{\mathrm{d}}(d)=\log \left(\frac{d^{\alpha}}{1+d^{\alpha}}\right),
$$

with $\alpha>0$, used by LargeVis [Tan $\left.{ }^{+} 16\right]$ and UMAP [MHM18, appendix C]. In fact, LargeVis and UMAP are equivalent to unconstrained MDE problems based on the log-one-plus attractive penalty and this repulsive penalty. The dimensionality 
reduction method t-SNE is almost equivalent to solving an MDE problem, except its objective encourages spreading via a non-separable function of the embedding distances. In practice, however, these three methods produce similar embeddings when their hyper-parameters are suitably adjusted [BBK20]. While there are many choices for repulsive (and attractive) penalties, in our experience, just a few simple functions, such as the ones described above, suffice.

Finally, we remark that it is possible to design distortion functions derived from weights that combine attractive and repulsive penalties. For example, the function

$$
f_{i j}\left(d_{i j}\right)=w_{i j} d_{i j}-\log \left(d_{i j}-\rho_{i j}\right)
$$

where $w_{i j}>0$ and $\rho_{i j}>0$, combines a linear penalty that attracts items $i$ and $j$ with a logarithmic barrier that repulses them. MDE problems using distortion functions like this one can be used to solve Euclidean placement and sphere-packing problems $[\mathrm{BV} 04, \S 8.7]$.

\subsection{Functions involving original distances}

In another class of MDE problems, for each item pair $(i, j) \in \mathcal{E}$, we are given a nonnegative number $\delta_{i j}$ representing the distance, deviation, or dissimilarity between items $i$ and $j$. A large value of $\delta_{i j}$ means $i$ and $j$ are very dissimilar, while $\delta_{i j}=0$ means items $i$ and $j$ are the same, or at least, very similar. We can think of $\delta_{i j}$ as the target distance for our embedding.

If the items can be represented by vectors (e.g., images, time-series data, bitstrings, and many other types of items), the original distances might be generated by a standard distance function, such as the Euclidean, Hamming, or earth-mover's distance. But the data $\delta_{i j}$ need not be metric; for example, they can fail to satisfy the triangle inequality $\delta_{i j} \leq \delta_{i k}+\delta_{k j}$, for $i<k<j,(i, j),(i, k),(k, j) \in \mathcal{E}$. This often happens when the $\delta_{i j}$ are scored by human experts.

Distortion functions. For problems involving distances, we consider distortion functions of the form

$$
f_{i j}\left(d_{i j}\right)=\ell\left(\delta_{i j}, d_{i j}\right), \quad(i, j) \in \mathcal{E},
$$

where $\ell: \mathbf{R}_{+} \times \mathbf{R}_{+} \rightarrow \mathbf{R}$ is a loss function. The loss function is nonnegative, zero for $d=\delta$, decreasing for $d<\delta$, and increasing for $d>\delta$. The embedding objective $E(X)$ is a measure of how closely the embedding distances match the original deviations. Perfect embedding corresponds to $E(X)=0$, which means $d_{i j}=$ $\delta_{i j}$, i.e., the embedding exactly preserves the original distances. This is generally 
impossible to achieve, for example when the original deviations are not a metric, and also in many cases even when it is. (For example, it is impossible to isometrically embed a four-point star graph with the shortest path metric into $\mathbf{R}^{m}$, for any $m$.) Instead, as in all MDE problems, we seek embeddings with low average distortion.

In these problems, we do not require the standardization constraint to encourage the embedding to spread out, though we may choose to enforce it anyway, for example to obtain uncorrelated features. When the standardization constraint is imposed, it is important to scale the original distances $\delta_{i j}$ so that their RMS value is not too far from the RMS value $d_{\text {nat }}(2.7)$ of the embedding distances $d_{i j}$.

When the original deviations $\delta_{i j}$ are Euclidean distances, it is sometimes possible to compute a perfect embedding (see $[\mathrm{BV} 04, \S 8.3 .3]$ and $\left[\mathrm{Lib}^{+} 14\right.$; Dok $\left.{ }^{+} 15\right]$ ). In general, however, MDE problems involving original distances are intractable. Nonetheless, the methods described in chapter 6 often produce satisfactory embeddings.

\subsubsection{Examples}

Quadratic loss. Perhaps the most obvious choice of loss function is the squared loss,

$$
\ell(\delta, d)=(\delta-d)^{2}
$$

The squared loss places more emphasis on preserving the distance between pairs with large original distances than small ones. In particular, when $\mathcal{E}$ contains all pairs and the feasible set is $\mathcal{S}$, a simple calculation shows that the least-squares $\mathrm{MDE}$ is equivalent to the problem

$$
\begin{array}{ll}
\text { minimize } & (1 / p) \sum_{(i, j) \in \mathcal{E}}-\delta_{i j} d_{i j} \\
\text { subject to } & X \in \mathcal{S}
\end{array}
$$

which we recognize as a linear distortion function, with coefficient the negative of the original distance. The objective of this problem gives outsized rewards to matching large original distances with large embedding distances.

The problem of finding an embedding that minimizes the least squares distortion (or simple variants of it) has been discussed extensively in the literature. A whole family of methods called multidimensional scaling is almost entirely concerned with (approximately) solving it [Kru64a; Kru64b; GLM96; CC00; BG03].

Weighted quadratic loss. A weighted quadratic loss is

$$
\ell(\delta, d)=\kappa(\delta)(\delta-d)^{2}
$$




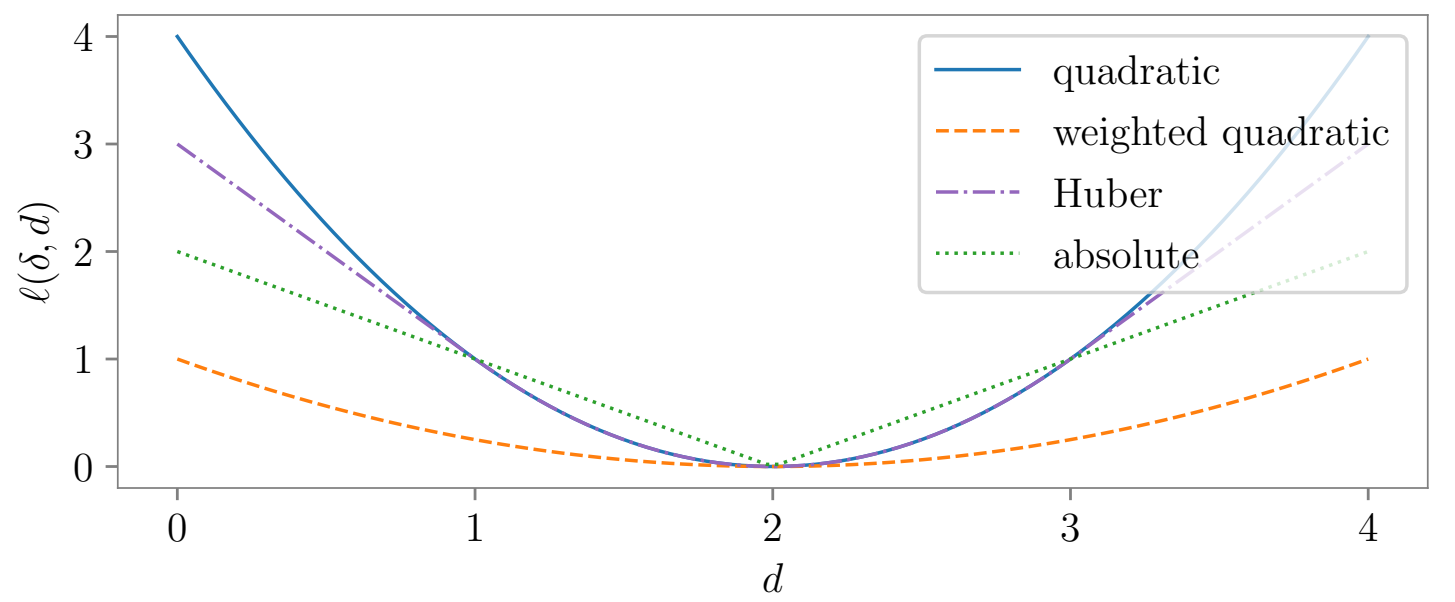

Figure 4.4: Basic losses involving distances. A quadratic loss, weighted quadratic (with $\kappa(\delta)=1 / \delta^{2}$ ), and a Huber loss (with threshold $\tau=1$ ), evaluated at $\delta=2$.

where $\kappa$ is a real function. If $\kappa$ is decreasing, the weighted quadratic loss places less emphasis on large distances $\delta$, compared to the quadratic loss. The choice $\kappa(\delta)=1 / \delta$ and $\mathcal{X}=\mathbf{R}^{n \times m}$ yields a method known as Sammon's mapping [Sam69], while $\kappa(\delta)=1 / \delta^{2}$ gives the objective function for the Kamada-Kawai algorithm for drawing graphs [KK89].

Huber loss. The Huber loss is

$$
\ell(\delta, d)= \begin{cases}(\delta-d)^{2} & |\delta-d| \leq \tau \\ \tau(2|\delta-d|-\tau) & |\delta-d|>\tau,\end{cases}
$$

where $\tau \in \mathbf{R}$ is a parameter [BV04, §6.1.2]. This loss can be thought of as a robust version of the quadratic loss, in that it is less sensitive to large residuals. This is illustrated in figure 4.4.

Absolute loss. The absolute loss is

$$
\ell(\delta, d)=|\delta-d| .
$$

MDE problems with this loss function have been referred to as robust MDS and robust EDM in the literature [CD06; ZXQ19]. 


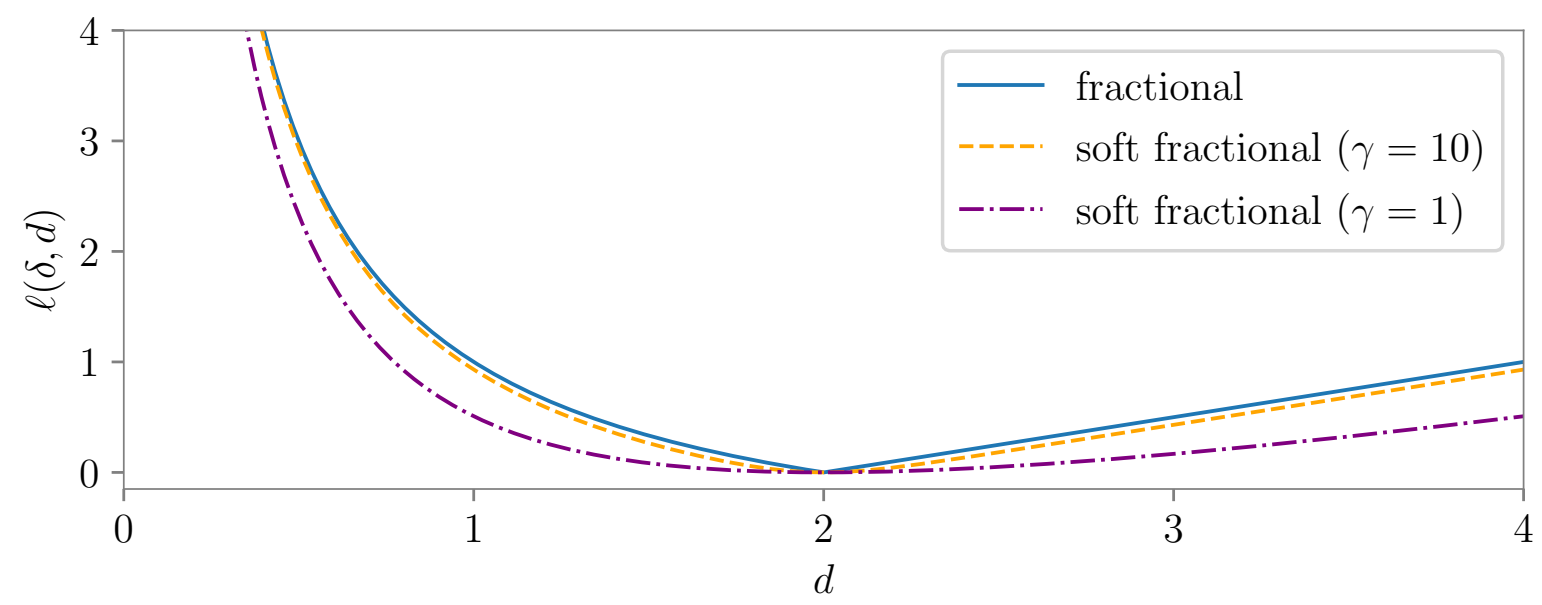

Figure 4.5: Fractional loss. The fractional and soft fractional losses, with $\delta=2$.

Logistic loss. Composing the logistic function with the absolute distortion gives a soft version of the absolute distortion,

$$
\ell(\delta, d)=\log \left(\frac{1+\exp |\delta-d|}{2}\right) .
$$

(Dividing the argument of the log by two has no effect on the MDE problem, but ensures that $\ell(\delta, \delta)=0$.)

Fractional loss. The fractional loss is defined as

$$
\ell(\delta, d)=\max \{\delta / d, d / \delta\}-1 .
$$

It is a barrier function: it approaches $+\infty$ as $d \rightarrow 0$, naturally encouraging the embedding vectors to spread out.

Soft fractional loss. A differentiable approximation of the fractional loss can be obtained by replacing the max function with the so-called softmax function,

$$
\ell(\delta, d)=\frac{1}{\gamma} \log \left(\frac{\exp (\gamma \delta / d)+\exp (\gamma d / \delta)}{2 \exp \gamma}\right),
$$

where $\gamma>0$ is a parameter. For large $\gamma$, this distortion function is close to the fractional distortion; for small $\gamma$ it is close to $\delta / d+d / \delta-2$. The soft fractional distortion is compared to the fraction distortion in figure 4.5. 


\subsection{Preprocessing}

In this section we discuss methods that can be used to create distortion functions, given some raw similarity or dissimilarity data on pairs of items. These preprocessing methods are analogous to feature engineering in machine learning, where raw features are converted to feature vectors and possibly transformed before further processing. As in feature engineering, preprocessing the raw data about items can have a strong effect on the final result, i.e., the embedding found.

Below, we discuss two preprocessing methods. The first method, based on neighborhoods, can be used to create distortion functions based on weights (and to choose which pairs to include in the sets $\mathcal{E}_{\text {sim }}$ and $\mathcal{E}_{\text {dis }}$ ). The second method, based on graph distances, is useful in creating distortion functions based on original deviations. These methods can also be combined, as explained later.

\subsubsection{Neighborhoods}

In some applications we have an original deviation $\delta_{i j}$ or weight $w_{i j}$ for every pair $(i, j)$, but we care mostly about identifying pairs that are very similar. These correspond to pairs where the deviation is small, or the weight is large. For raw data given as deviations, the neighborhood of an item $i$ is

$$
\mathcal{N}\left(i ; \delta_{i}^{\text {thres }}\right)=\left\{j \mid \delta_{i j} \leq \delta_{i}^{\text {thres }}\right\}
$$

and for raw data given as weights, the neighborhood is

$$
\mathcal{N}\left(i ; w_{i}^{\text {thres }}\right)=\left\{j \mid w_{i j} \geq w_{i}^{\text {thres }}\right\},
$$

where $\delta_{i}^{\text {thres }}$ and $w_{i}^{\text {thres }}$ are threshold values.

If item $j$ is in the neighborhood of item $i$ (and $j \neq i$ ), we say that $j$ is a neighbor of $i$. Note that this definition is not symmetric: $j \in \mathcal{N}(i)$ does not imply $i \in \mathcal{N}(j)$. The graph whose edges connect neighbors is called a neighborhood graph, with edges

$$
\mathcal{E}_{\text {sim }}=\{(i, j) \mid 1 \leq i<j \leq n, j \in \mathcal{N}(i) \text { or } i \in \mathcal{N}(j)\}
$$

Building the neighborhood graph. The neighborhood graph depends on the threshold values $\delta_{i}^{\text {thres }}$ and $w_{i}^{\text {thres }}$. A simple option is to use the same threshold $\delta^{\text {thres }}$ or $w^{\text {thres }}$ for all items, but we can also use different thresholds for different items. An effective way to do this is based on the nearest neighbors of each item. For original data given as deviations, we choose

$$
\delta_{i}^{\text {thres }}=\sup \{\delta|| \mathcal{N}(i ; \delta) \mid \leq k\}
$$


and analogously for weights; that is, we choose the thresholds so that each item has $k$ neighbors, where $k$ is a parameter that is much smaller than $n$. More sophisticated methods of building neighborhood graphs are possible, such as the one proposed in [CPZ05], but this simple choice often works very well in practice.

Computing the $k$-nearest neighbors of each item is expensive. In almost all applications, however, it suffices to carry out this computation approximately. Approximate nearest neighbors can be computed cheaply using algorithms such as the one introduced in [DCL11], which has a reported empirical complexity of $O\left(n^{1.14}\right)$, or the methods surveyed in [AIR18]. Python libraries such as pynndescent [McI20a] and annoy [Ber20b] make these computations straightforward in practice.

Choosing dissimilar items. A natural choice for the set of dissimilar items is

$$
\mathcal{E}_{\text {dis }}=\left\{(i, j) \mid(i, j) \notin \mathcal{E}_{\text {sim }}\right\},
$$

i.e., all pairs of non-neighbors. This says that all items that are not similar are dissimilar. If $n$ is large, this choice is untenable because $\mathcal{E}_{\text {sim }} \cup \mathcal{E}_{\text {dis }}$ will include all $n(n-1) / 2$ pairs. A practical alternative is to randomly select a subset of nonneighbors, and include only these edges in $\mathcal{E}_{\text {dis }}$. The size of $\mathcal{E}_{\text {dis }}$ affects how spread out the embedding is; in our experience, an effective choice is to sample $\left|\mathcal{E}_{\text {sim }}\right|$ nonneighbors uniformly at random. (If a standardization constraint or anchor constraint is imposed, we can also take $\mathcal{E}_{\text {dis }}=\emptyset$.) Several well-known embedding methods, such as word2vec, LargeVis, and UMAP, choose dissimilar pairs via random sampling (these methods call this "negative sampling") [Mik ${ }^{+} 13$; $\left.\mathrm{Tan}^{+} 16 ; \mathrm{MHM} 18 ; \mathrm{BBK} 20\right]$.

Other choices are possible. Instead of including all pairs not in $\mathcal{E}_{\text {sim }}$, we can take $\mathcal{E}_{\text {dis }}$ to only include pairs of items with a large original distance (or small original weight). This can yield embeddings in which similar items are tightly clustered together, and are far away from dissimilar items.

Choosing weights. After constructing $\mathcal{E}_{\text {sim }}$ and $\mathcal{E}_{\text {dis }}$, we assign distortion functions to edges. We have already discussed how to choose penalty functions, in $\S 4$.1. Here we discuss the choice of weights $w_{i j}$ in the distortion functions.

The simplest choice of weights is $w_{i j}=+1$ for $(i, j) \in \mathcal{E}_{\text {sim }}$ and $w_{i j}=-1$ for $(i, j) \in \mathcal{E}_{\text {dis }}$. Though exceedingly simple, this weighting often works very well in practice. Another effective choice takes $w_{i j}=+2$ if $i \in \mathcal{N}(j)$ and $j \in \mathcal{N}(i), w_{i j}=+1$ if $i \in \mathcal{N}(j)$ but $j \notin \mathcal{N}(i)$ (or vice versa), and $w_{i j}=-1$ if $(i, j) \in \mathcal{E}_{\text {dis. }}$.

The weights can also depend on the raw weights or deviations, as in

$$
w_{i j}=\exp \left(-\delta_{i j}^{2} / \sigma^{2}\right)
$$


where $\sigma$ is a positive parameter. In this preprocessing step, the thresholds and parameters such as $\sigma$ are chosen, usually by experimentation, to get good results.

Sparsity. Preprocessing methods based on neighborhoods focus on the local structure in the raw data. Roughly speaking, we trust the original data to indicate pairs of items that are similar, but less so which items are dissimilar. These preprocessing steps typically yield a sparse graph, with many fewer than $n(n-1) / 2$ edges. In many cases the average degree of the vertices in the graph is modest, say, on the order of 10 , in which case number of edges $p$ is a modest multiple of the number of items $n$. This makes problems with $n$ large, say $10^{6}$, tractable; it would not be practical to handle all $10^{12}$ edges in a full graph, but handling $10^{7}$ edges is quite tractable.

\subsubsection{Graph distance}

The previous preprocessing step focuses on the local structure of the raw data, and generally yields a sparse graph; we can also use sparse original data to create a full graph. We consider the case with original deviations $\delta_{i j}$, for some or possibly all pairs $(i, j)$. We replace these with the graph distance $\tilde{\delta}_{i j}$ between vertices $i$ and $j$, defined as the minimum sum of deviations $\delta_{i j}$ over all paths between $i$ and $j$. (If the original graph is complete and the original deviations satisfy the triangle inequality, then this step does nothing, and we have $\tilde{\delta}_{i j}=\delta_{i j}$.)

We mention that the graph distances can be computed efficiently, using standard algorithms for computing shortest paths (such as Dijkstra's algorithm, or breadthfirst search for unweighted graphs): simply run the shortest-path algorithm for each node in the graph. While the time complexity of computing all $n(n-1) / 2$ graph distances is $\tilde{O}\left(n^{2}+n p\right)$, the computation is embarrassingly parallel across the nodes, so in practice this step does not take too long. (For example, on a standard machine with 8 cores, 300 million graph distances can be computed in roughly one minute.)

Sparsity. This operation yields a complete graph with $n(n-1) / 2$ edges (assuming the original graph is connected). When $n$ is large, the complete graph might be too large to fit in memory. To obtain sparser graphs, as a variation, we can randomly sample a small fraction of the $n(n-1) / 2$ graph distances, and use only the edges associated with these sampled graph distances. This variation can be computed efficiently, without storing all $n(n-1) / 2$ graph distances in memory, by iteratively sampling the graph distances for each node.

Another sparsifying variation is to limit the length of paths used to determine the new deviations. For example, we can define $\tilde{\delta}_{i j}$ as the minimum sum of original 
deviations over all paths between $i$ and $j$ of length up to $L$. The parameter $L$ would be chosen experimentally.

Example. As a simple example, we use this preprocessing step to create MDE problems for visualizing graphs (similar to the examples in §2.5). Figure 4.6 plots standardized embeddings of a chain graph, a cycle graph, a star graph, and a binary tree on $n=20$ vertices, obtained by solving MDE problems with the weighted quadratic loss and original distances $\delta_{i j}$ given by the graph distance.

\subsubsection{Other methods}

Preprocessing based on shortest path distances and neighborhoods are readily combined. As an example, we might first sparsify the original data, forming a neighborhood graph. Then we define distortion functions as $f_{i j}\left(d_{i j}\right)=d_{i j}^{2}$ if $(i, j)$ is a pair of neighbors, and $f_{i j}=(1 / 2) d_{i j}^{2}$ if $(i, j)$ have distance two on the neighborhood graph.

Another natural preprocessing step is based on preference data. Here, we are given a set of comparisons between items. This kind of data is often readily available (e.g., surveys), though we mention the literature on preference elicitation [SCL13] and aggregation [Arr50; BT52; Pla75; Luc12; FV86] is rich and goes back centuries [EK10], with a number of advances continuing today [Joa02; Dwo ${ }^{+} 01$; VAD08]. As a simple example, imagine we have a set of rankings, expressing whether item $i$ is preferred to item $j$. In this case, it can make sense to set the deviations $\delta_{i j}$ to any increasing function of the average distance between the ranks of items $i$ and $j$. As another example, we might have a set of survey responses, and some of these responses might indicate items $i$ and $j$ are more similar to each other than they are to item $k$. There are many ways to create weights from these kinds of comparisons. A simple one is to set the weights $w_{i j}$ to the number of times items $i$ and $j$ are indicated as the most similar pair, minus the number of times they are not. Thus, $\mathcal{E}_{\text {sim }}$ contains all pairs with $w_{i j} \geq 0$, while $\mathcal{E}_{\text {dis }}$ contains those with $w_{i j}<0$.

Evidently there are many reasonable preprocessing steps that can be used. As in feature engineering for machine learning, there is some art in the choice of preprocessing. But also as in feature engineering, a few simple preprocessing steps, such as the ones described above, often work very well. 

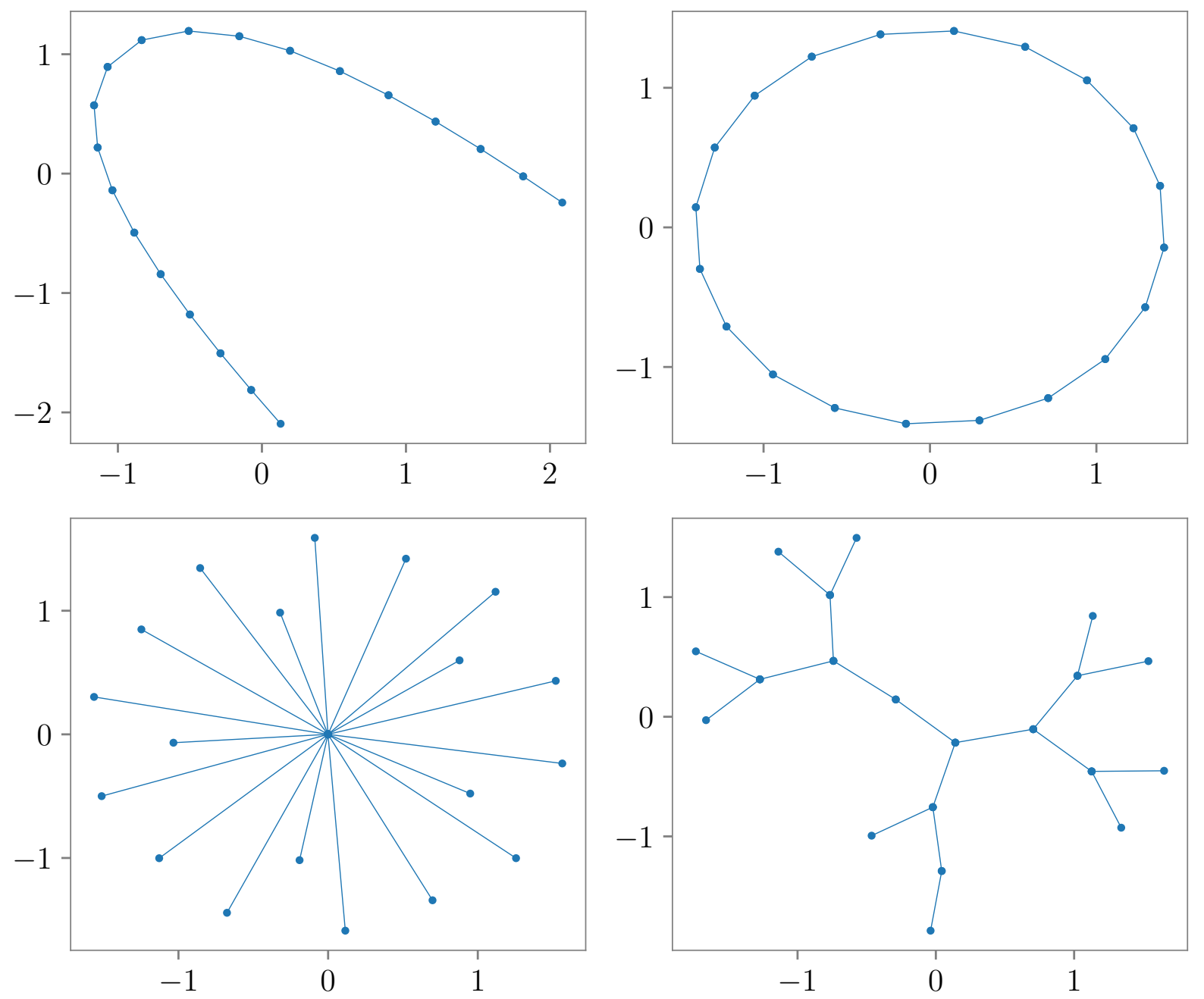

Figure 4.6: Graph layout with weighted quadratic loss. Standardized embeddings of a chain graph, a cycle graph, a star graph, and a binary tree, using the weighted quadratic loss derived from graph distances, with $\kappa(\delta)=1 / \delta^{2}$. 


\section{Part II}

\section{Algorithms}




\section{Chapter 5}

\section{Stationarity Conditions}

In this chapter we give conditions for an embedding $X \in \mathcal{X} \subseteq \mathbf{R}^{n \times m}$ to be stationary for MDE problems with differentiable average distortion; these conditions are necessary, but not sufficient, for an embedding to be optimal. The stationarity conditions presented here will guide our development of algorithms for computing minimum-distortion embeddings, as we will see in the next chapter.

The stationarity conditions can be expressed as a certain matrix $G \in \mathbf{R}^{n \times m}$ being zero. This matrix $G$ is the projection of the gradient $\nabla E(X)$ onto the (negative) tangent cone of $\mathcal{X}$ at $X$, and it will play a crucial rule in the algorithms presented in chapter 6. We give explicit expressions for $G$ for the constraint sets $\mathcal{C}(2.3), \mathcal{A}(2.4)$, and $\mathcal{S}(2.5)$, in $\S 5.1, \S 5.2$. 55.3 .

Below, we develop and explain this simple stationarity condition, interpreting $-G$ as the steepest feasible descent direction for $E$ at $X$ for the constraint $X \in \mathcal{X}$. We start by giving an expression for the gradient of $E$.

Gradient. The gradient of the average distortion is

$$
\nabla E(X)=(1 / p) A C A^{T} X, \quad C=\operatorname{diag}\left(f_{1}^{\prime}\left(d_{1}\right) / d_{1}, \ldots, f_{p}^{\prime}\left(d_{p}\right) / d_{p}\right),
$$

when $d_{k}>0$ for all $k$, i.e., $x_{i} \neq x_{j}$ for $(i, j) \in \mathcal{E}$; here, $A \in \mathbf{R}^{n \times p}$ is the incidence matrix (2.2). The average distortion is differentiable for all embeddings if $f_{k}^{\prime}(0)=0$ for $k=1, \ldots, p$. In this case we replace $f_{k}^{\prime}\left(d_{k}\right) / d_{k}$ with $f_{k}^{\prime \prime}(0)$ in our expression for $C$ when $d_{k}=0$. In the sequel we will ignore these points of nondifferentiability, which do not arise in practice with reasonable choices of distortion functions.

We observe that if the distortion functions are nondecreasing, i.e., the objective is attractive, the matrix $C$ has nonnegative entries, and the matrix $A C A^{T}$ is a Laplacian matrix for a graph $(\mathcal{V}, \mathcal{E})$ with edge weights given by the diagonal of $C$. 
Tangents. A matrix $V \in \mathbf{R}^{n \times m}$ is tangent to $\mathcal{X}$ at $X \in \mathcal{X}$ if for small $h$,

$$
\operatorname{dist}(X+h V, \mathcal{X})=o(h),
$$

where dist is the Euclidean distance of its first argument to its second. We write $\mathcal{T}_{X}(\mathcal{X})$ to denote the set of tangents to $\mathcal{X}$ at $X$. For smooth constraint sets, such as $\mathcal{C}, \mathcal{A}$, and $\mathcal{S}$, this set is a subspace; more generally, it is a cone. In the context of optimization algorithms, a direction $V \in \mathcal{T}_{X}(\mathcal{X})$ is called a feasible direction at $X$, for the constraint $X \in \mathcal{X}$.

Feasible descent directions. The first-order Taylor approximation of $E(X+V)$ at $X$ is

$$
\hat{E}(X+V ; X)=E(X)+\operatorname{tr}\left(\nabla E(X)^{T} V\right),
$$

where the term $\operatorname{tr}\left(\nabla E(X)^{T} V\right)$ is the directional derivative of $E$ at $X$ in the direction $V$. The directional derivative gives the approximate change in $E$ for a small step $V$; the direction $V$ is a descent direction if $\operatorname{tr}\left(\nabla E(X)^{T} V\right)<0$, in which case $\hat{E}(X+$ $V ; X)<E(X)$ for small $V$ [BV04, §9.4]. A direction $V$ is called a feasible descent direction if it is a feasible direction and a descent direction, i.e.,

$$
V \in \mathcal{T}_{X}(\mathcal{X}), \quad \operatorname{tr}\left(\nabla E(X)^{T} V\right)<0 .
$$

This defines a cone of feasible descent directions. It is empty if $X \in \mathcal{X}$ is a stationary point for the MDE problem. (We mention that the negative gradient is evidently a descent direction for $E$, but it is not necessarily feasible for the constraint.)

Steepest feasible descent direction. The steepest feasible descent direction (with respect to the Frobenius norm) is defined as the (unique) solution of the problem

$$
\begin{array}{ll}
\operatorname{minimize} & \operatorname{tr}\left(\nabla E(X)^{T} V\right)+\frac{1}{2}\|V\|_{F}^{2} \\
\text { subject to } & V \in \mathcal{T}_{X}(\mathcal{X})
\end{array}
$$

(see [HUL93, chapter VIII, §2.3]). When the solution is 0 , the cone of feasible descent directions is empty, i.e., $X$ is a stationary point of the MDE problem. We will denote the steepest feasible descent direction as $-G$. (We use the symbol $G$ since when $\mathcal{X}=\mathbf{R}^{n \times m}$, it coincides with the gradient $\nabla E(X)$.)

Projected gradient. We can express the (negative) steepest feasible descent direction as

$$
G=\Pi_{\mathcal{T}_{X}}(\nabla E(X)),
$$


the Euclidean projection of the gradient $\nabla E(X)$ onto the (negative) tangent cone of $\mathcal{X}$ at $X$. That is, $G$ is the solution of

$$
\begin{array}{ll}
\text { minimize } & \frac{1}{2}\|\nabla E(X)-V\|_{F}^{2} \\
\text { subject to } & V \in-\mathcal{T}_{X}(\mathcal{X}),
\end{array}
$$

We will refer to $G$ as the projected gradient of $E$ at $X \in \mathcal{X}$. When the set of tangents to $\mathcal{X}$ at $X$ is a subspace, the constraint can be written more simply as $V \in \mathcal{T}_{X}(\mathcal{X})$.

Stationarity condition. The stationarity condition for a constrained MDE problem is simply

$$
G=0 .
$$

This condition is equivalent to the well-known requirement that the negative gradient of the objective lie in the normal cone of the constraint set at $X$ [NW06, §12.7].

Stopping criterion. The stationarity condition (5.3) leads to a natural stopping criterion for iterative optimization algorithms for the MDE problem. We can interpret $G$ as a residual for the optimality condition; algorithms should seek to make the Frobenius norm of the residual very small, if not exactly 0 . This suggests the termination condition

$$
\|G\|_{F} \leq \epsilon,
$$

where $\epsilon$ is a given threshold or tolerance. The threshold $\epsilon$ can be a small constant, such as $10^{-5}$, or it may depend on problem parameters such as $n, m$, or $p$.

We use the stopping criterion (5.4) for the algorithm we present in chapter 6. Of course, a stationary embedding need not be a global or even local minimum, but we find that using this stopping criterion (along with our algorithm) consistently yields good embeddings in practice, as long as the MDE problem is well-posed.

In the following sections we derive explicit expressions for the tangent space $\mathcal{T}_{X}(\mathcal{X})$, and the projected gradient $G$, at a point $X$, for our three specific constraint sets $\mathcal{C}$, $\mathcal{A}$, and $\mathcal{S}$. We also derive expressions for the optimal dual variables, though we will not use these results in the sequel. We start each section by recalling the definition of the constraint set (the definitions were given in §2.4).

\subsection{Centered MDE problems}

The set of centered embeddings is

$$
\mathcal{C}=\left\{X \mid X^{T} \mathbf{1}=0\right\} .
$$


The tangent space of $\mathcal{C}$ is the set of centered directions,

$$
\mathcal{T}_{X}(\mathcal{C})=\left\{V \mid V^{T} \mathbf{1}=0\right\} .
$$

From the expression (5.1) for the gradient, it can be seen that $\nabla E(X) \in \mathcal{T}_{X}(\mathcal{C})$ for $X \in \mathcal{C}$. Therefore, the projection onto the tangent space is the identity mapping, i.e.,

$$
\Pi_{\mathcal{T}_{X}}(\nabla E(X))=\nabla E(X)
$$

yielding the familiar stationarity condition

$$
G=\nabla E(X)=0 .
$$

In the mechanical interpretation, the rows of the gradient $\nabla E(X)=1 / p\left(A C A^{T} X\right)$ are the net force on the points due to the springs, and the stationarity condition (5.6) says that the points are at equilibrium. To see this, note that the matrix $C A^{T} X$ gives the forces between items incident to the same edge. The $p$ rows of $C A^{T} X$ are

$$
f_{k}^{\prime}\left(d_{k}\right) \frac{x_{i(k)}-x_{j(k)}}{d_{k}}, \quad k=1, \ldots, p
$$

i.e., $x_{i(k)}$ experiences a force of magnitude $\left|f_{k}^{\prime}\left(d_{k}\right)\right|$ in the direction $\operatorname{sign}\left(f_{k}^{\prime}\left(d_{k}\right)\right)\left(x_{i(k)}-\right.$ $\left.x_{j(k)}\right) / d_{k}$, due to its connection to $x_{j(k)}$.

\subsection{Anchored MDE problems}

The set of anchored embeddings is

$$
\mathcal{A}=\left\{X \mid x_{i}=x_{i}^{\text {given }}, i \in \mathcal{K}\right\},
$$

where $\mathcal{K} \subseteq \mathcal{V}$ is the index set of anchored vertices. The tangent space of an anchor constraint set $\mathcal{A}$ at a point $X \in \mathcal{A}$ is the set of directions that preserve the values of the anchors, i.e.,

$$
\mathcal{T}_{X}(\mathcal{A})=\left\{V \mid v_{i}=0, i \in \mathcal{K}\right\},
$$

where $v_{1}^{T}, v_{2}^{T}, \ldots, v_{n}^{T}$ are the rows of $V$. The projection onto the tangent space zeros out the rows corresponding to the anchors:

$$
\Pi_{\mathcal{T}_{X}}(\nabla E(X))=P \nabla E(X), \quad P=\left[\begin{array}{c}
p_{1}^{T} \\
p_{2}^{T} \\
\vdots \\
p_{n}^{T}
\end{array}\right], \quad p_{i}= \begin{cases}e_{i} & i \notin \mathcal{K} \\
0 & \text { otherwise }\end{cases}
$$


where $P \in \mathbf{R}^{n \times n}$ is a masking matrix and $e_{i} \in \mathbf{R}^{n}$ is the $i$ th standard basis vector. Therefore, the stationarity condition

$$
G=P \nabla E(X)=0
$$

says that the rows of the gradient corresponding to the free vertices must be zero.

\subsubsection{Dual variables}

The stationarity condition (5.8) can also be seen via Lagrange multipliers. A simple calculation shows that for a stationary embedding $X \in \mathcal{A}$, the optimal dual variables $\lambda_{i} \in \mathbf{R}^{m}$ (associated with the constraints $x_{i}=x_{i}^{\text {given }}$ ) must satisfy

$$
\lambda_{i}=-\nabla E(X)_{i}
$$

In the mechanical interpretation of an MDE problem, the dual variables $\lambda_{i}$ are the forces on the anchored vertices due to the constraint $X \in \mathcal{A}$.

\subsection{Standardized MDE problems}

The set of standardized embeddings is

$$
\mathcal{S}=\left\{X \mid(1 / n) X^{T} X=I, X^{T} \mathbf{1}=0\right\} .
$$

The tangent space to $\mathcal{S}$ at $X \in \mathcal{S}$ is the subspace

$$
\mathcal{T}_{X}(\mathcal{S})=\left\{V \mid V^{T} \mathbf{1}=0, X^{T} V+V^{T} X=0\right\},
$$

as can be seen by differentiating the constraint $(1 / n) X^{T} X=I$. The projected gradient $G$ of $E$ at $X \in \mathcal{S}$ is therefore the solution to the linearly constrained least squares problem

$$
\begin{array}{ll}
\operatorname{minimize} & \frac{1}{2}\|\nabla E(X)-V\|_{F}^{2} \\
\text { subject to } & V^{T} \mathbf{1}=0, \quad V^{T} X+X^{T} V=0
\end{array}
$$

with variable $V \in \mathbf{R}^{n \times m}$. This problem has the simple solution

$$
\Pi_{\mathcal{T}_{X}}(\nabla E(X))=\nabla E(X)-(1 / n) X \nabla E(X)^{T} X .
$$

This means that $X \in \mathcal{S}$ is stationary for a standardized MDE problem if

$$
G=\nabla E(X)-(1 / n) X \nabla E(X)^{T} X=0 .
$$


The solution (5.10) is readily derived with Lagrange multipliers. The Lagrangian of $(5.9)$ is

$$
\mathcal{L}(V, \Lambda)=\frac{1}{2}\left(\|\nabla E(X)\|_{F}^{2}-2 \operatorname{tr}\left(\nabla E(X)^{T} V\right)+\|V\|_{F}^{2}\right)+\operatorname{tr}\left(\Lambda^{T}\left(V^{T} X+X^{T} V\right)\right),
$$

where $\Lambda \in \mathbf{R}^{m \times m}$ is the multiplier (we do not need a multiplier for the centering constraint $X^{T} \mathbf{1}=0$, since it can be imposed without loss of generality). The optimal $V$ and $\Lambda$ must satisfy

$$
\nabla_{V} \mathcal{L}(V, \Lambda)=-\nabla E(X)+V+X\left(\Lambda^{T}+\Lambda\right)=0
$$

Using $V^{T} X+X^{T} V=0$ and $(1 / n) X^{T} X=I$, we obtain $\Lambda^{T}+\Lambda=(1 / n) \nabla E(X)^{T} X$, from which (5.10) follows. From the expression of the gradient (5.1) and the fact that $X^{T} \mathbf{1}=0$, it is easy to check that $G$ satisfies $G^{T} \mathbf{1}=0$.

\subsubsection{Dual variables}

We can directly derive the stationarity condition (5.11) using Lagrange multipliers, without appealing to the projected gradient. The Lagrangian of the MDE problem with standardization constraint is

$$
\mathcal{L}(X, \Lambda)=E(X)+(1 / n) \operatorname{tr}\left(\Lambda^{T}\left(I-X^{T} X\right)\right),
$$

where $\Lambda=\Lambda^{T} \in \mathbf{R}^{m \times m}$ is the Lagrange multiplier associated with the constraint $I-(1 / n) X^{T} X=0$ (again, we omit a multiplier for the centering constraint). The optimal multiplier $\Lambda$ must satisfy

$$
\nabla_{X} \mathcal{L}(X, \Lambda)=\nabla E(X)-(2 / n) X \Lambda=0
$$

Multiplying by $X^{T}$ on the left and using $(1 / n) X^{T} X=I$ yields

$$
\Lambda=\frac{1}{2} \nabla E(X)^{T} X=\frac{1}{2 p} X^{T} A C A^{T} X
$$

Substituting this expression for $\Lambda$ into (5.12), we obtain the stationarity condition (5.11).

We can interpret the rows of $-(2 / n) X \Lambda$ as the forces put on the points by the constraint $X \in \mathcal{S}$. The stationarity condition (5.11) is again that the total force on each point is zero, including both the spring forces from other points, and the force due to the constraint $X \in \mathcal{S}$. 
Finally, we observe that the multiplier $\Lambda$ satisfies the interesting property

$$
\operatorname{tr}(\Lambda)=\frac{1}{p} \operatorname{tr}\left(X^{T} A C A^{T} X\right) / 2=\frac{1}{p} \sum_{k=1}^{p} f_{k}^{\prime}\left(d_{k}\right) d_{k} / 2 .
$$

In other words, $\operatorname{tr}(\Lambda)$ equals the average distortion of an MDE problem with distortion functions $\tilde{f}_{k}\left(d_{k}\right)=f_{k}^{\prime}\left(d_{k}\right) d_{k} / 2$ (and the same items and pairs as the original problem). When the distortion functions are weighted quadratics, this MDE problem coincides with the original one, i.e., $\operatorname{tr}(\Lambda)=E(X)$.

Quadratic problems. For quadratic MDE problems, which were studied in chapter 3, the matrix (1/2)AC $A^{T}$ is the matrix $L$, so from (5.13) the optimal dual variable satisfies

$$
L X=(p / n) X \Lambda .
$$

Substituting the optimal value of $X$ into this equation, we see that the optimal dual variable is diagonal, and its entries are eigenvalues of $L$. In particular,

$$
\Lambda=(n / p) \operatorname{diag}\left(\lambda_{1}, \ldots, \lambda_{m}\right)
$$

for $j>m$, or

$$
\Lambda=(n / p) \operatorname{diag}\left(\lambda_{1}, \ldots, \lambda_{j-1}, \lambda_{j+1}, \ldots, \lambda_{m+1}\right) .
$$

for $j \leq m$. Notice that in both cases, $\operatorname{tr}(\Lambda)=E(X)$. 


\section{Chapter 6}

\section{Algorithms}

MDE problems are generally intractable: there are no efficient algorithms for finding exact solutions, except in special cases such as when the distortion functions are quadratic, as described in chapter 3, or when the distortion functions are convex and nondecreasing and the embedding is anchored. In this chapter we describe two heuristic algorithms for approximately solving MDE problems. The first algorithm is a projected quasi-Newton algorithm, suitable for problems that fit in the memory of a single machine. The second algorithm is a stochastic method that builds on the first to scale to much larger problems. While we cannot guarantee that these algorithms find minimum-distortion embeddings, in practice we observe that they reliably find embeddings that are good enough for various applications.

We make a few assumptions on the MDE problem being solved. We assume that the average distortion is differentiable, which we have seen occurs when the embedding vectors are distinct. In practice, this is almost always the case. We have also found that the methods described in this chapter work well when this assumption does not hold, even in the case when the distortion functions themselves are nondifferentiable.

Our access to $E$ is mediated by a first-order oracle: we assume that we can efficiently evaluate the average distortion $E(X)$ and its gradient $\nabla E(X)$ at any $X \in \mathcal{X}$. Additionally, we also assume that projections onto the tangent cone and constraint set exist, and that the associated projection operators $\Pi_{\mathcal{T}_{X}}(Z)$ and $\Pi_{\mathcal{X}}(Z)$ can be efficiently evaluated for $Z \in \mathbf{R}^{n \times m}$. Our algorithms can be applied to any MDE problem for which these four operations, evaluating $E, \nabla E, \Pi_{\mathcal{T}_{X}}$, and $\Pi_{\mathcal{X}}$, can be efficiently carried out. We make no other assumptions.

We describe the projected quasi-Newton algorithm in $\S 6.1$. The algorithm is an iterative feasible descent method, meaning that it produces a sequence of iterates 
$X_{k} \in \mathcal{X}, k=0,1, \ldots$, with $E\left(X_{k}\right)$ decreasing. In $\S 6.2$ we describe a stochastic proximal method suitable for very large MDE problems, with billions of items and edges. This method uses the projected quasi-Newton algorithm to approximately solve a sequence of smaller, regularized MDE subproblems, constructed by sampling a subset of the edges. Unlike traditional stochastic methods, we use very large batch sizes, large enough to fill the memory of the hardware used to solve the subproblems. We will study the performance of our algorithms when applied to a variety of MDE problems, using a custom software implementation, in the following chapter.

\subsection{A projected quasi-Newton algorithm}

In this section we describe a projected quasi-Newton method for approximately solving the MDE problem

$$
\begin{array}{ll}
\text { minimize } & E(X) \\
\text { subject to } & X \in \mathcal{X},
\end{array}
$$

with matrix variable $X \in \mathbf{R}^{n \times m}$.

Each iteration involves computing the gradient $\nabla E\left(X_{k}\right)$ and its projection $G_{k}=$ $\Pi_{\mathcal{T}_{X}}\left(\nabla E\left(X_{k}\right)\right)$ onto the tangent space (or cone) of the constraint set at the current iterate. A small history of the projected gradients is stored, which is used to generate a quasi-Newton search direction $V_{k}$. After choosing a suitable step length $t_{k}$ via a line search, the algorithm steps along the search direction, projecting $X_{k}+t_{k} V_{k}$ onto $\mathcal{X}$ to obtain the next iterate. We use the stopping criterion (5.4), terminating the algorithm when the residual norm $\left\|G_{k}\right\|_{F}$ is sufficiently small (or when the iteration number $k$ exceeds a maximum iteration limit). We emphasize that this algorithm can be applied to any MDE problem for which we can efficiently evaluate the average distortion, its gradient, and the operators $\Pi_{\mathcal{T}_{X}}$ and $\Pi_{\mathcal{X}}$.

Below, we explain the various steps of our algorithm. Recall that we have already described the computation of the gradient $\nabla E(X)$, in (5.1); likewise, the projected gradients for these constraint sets are given in (5.5), (5.7), and (5.10). We first describe the computation of the quasi-Newton search direction $V_{k}$ and step length $t_{k}$ in each iteration. Next we describe the projections onto the constraint sets $\mathcal{C}$, $\mathcal{A}$, and $\mathcal{S}$. In all three cases the overhead of computing the projections is negligible compared to the cost of computing the average distortion and its gradient. Finally we summarize the algorithm and discuss its convergence. 


\subsubsection{Search direction}

In each iteration, the quasi-Newton search direction $V_{k}$ is computed using a procedure that is very similar to the one used in the limited-memory Broyden-FletcherGoldfarb-Shanno (L-BFGS) algorithm [Noc80], except we use projected gradients instead of gradients. For completeness, we briefly describe the algorithm here; a more detailed discussion (in terms of gradients) can be found in [NW06, §7.2]. In what follows, for a matrix $X \in \mathbf{R}^{n \times m}$, we write vec $X$ to denote its representation as a vector in $\mathbf{R}^{n m}$ containing the entries of $X$ in some fixed order.

In iteration $k$, the search direction $V_{k}$ is computed as

$$
\operatorname{vec} V_{k}=-B_{k}^{-1} \operatorname{vec} G_{k},
$$

where $B_{k} \in \mathbf{S}_{++}^{n m}$ is a positive definite matrix. In particular, $B_{k}^{-1}$ is given by the recursion

$$
B_{j+1}^{-1}=\left(I-\frac{s_{j} y_{j}^{T}}{y_{j}^{T} s}\right) B_{j}^{-1}\left(I-\frac{y_{j} s_{j}^{T}}{y_{j}^{T} s_{j}}\right)+\frac{s_{j} s_{j}^{T}}{y_{j}^{T} s_{j}}, \quad j=k-1, \ldots, k-M,
$$

using $B_{k-M}=\gamma_{k} I$; the positive integer $M$ is the memory size, and

$$
s_{j}=\operatorname{vec}\left(X_{j+1}-X_{j}\right), \quad y_{j}=\operatorname{vec}\left(G_{j+1}-G_{j}\right), \quad \gamma_{k}=\frac{y_{k-1}^{T} s_{k-1}}{y_{k-1}^{T} y_{k-1}} .
$$

When $M \geq k$ and $\mathcal{X}=\mathbf{R}^{n \times m}$, the matrix $B_{k}$ is the BFGS approximation to the Hessian of $E$ (viewed as a function from $\mathbf{R}^{n m}$ to $\mathbf{R}$ ), which satisfies the secant condition $B_{k} s_{k-1}=y_{k-1}$ [Bro70; Fle70; Gol70; Sha70]. More generally, $B_{k}$ approximates the curvature of the restriction of $E$ to $\mathcal{X}$. In practice the matrices $B_{k}^{-1}$ are not formed explicitly, since the matrix-vector product $B_{k}^{-1}$ vec $G_{k}$ can be computed in $O(n m M)+O\left(M^{3}\right)$ time [NW06, $\left.\S 7.2\right]$. (The memory $M$ is typically small, on the order of 10 , so the second term is usually negligible.)

After computing the search direction, we form the intermediate iterate

$$
X_{k+1 / 2}=X_{k}+t_{k} V_{k}
$$

where $t_{k}>0$ is a step length. The next iterate is obtained by projecting the intermediate iterate onto $\mathcal{X}$, i.e.,

$$
X_{k+1}=\Pi_{\mathcal{X}}\left(X_{k+1 / 2}\right) .
$$

We mention for future reference that the intermediate iterates satisfy

$$
X_{k+1 / 2} \in X_{k}+\operatorname{span}\left\{G_{k}, G_{k-1}, \ldots, G_{k-m}, s_{k-1}, \ldots, s_{k-m}\right\} .
$$


(see [JD17, §A2]). Note in particular that if $G_{1}, G_{2}, \ldots, G_{k}$ and $X_{1}, X_{2}, \ldots, X_{k}$ are all centered, then $X_{k+1 / 2}$ is also centered (i.e., $X_{k+1 / 2}^{T} \mathbf{1}=0$ ).

We choose the step length $t_{k}$ via a line search, to satisfy the modified strong Wolfe conditions

$$
\begin{aligned}
& E\left(\Pi_{\mathcal{X}}\left(X_{k+1 / 2}\right)\right) \leq E\left(X_{k}\right)+c_{1} t_{k} \operatorname{tr}\left(G_{k}^{T} V_{k}\right), \\
& \left.\mid \operatorname{tr}\left(G_{k+1}^{T} V_{k}\right)\right)\left|\leq c_{2}\right| \operatorname{tr}\left(G_{k}^{T} V_{k}\right) \mid
\end{aligned}
$$

where $0<c_{1}<c_{2}<1$ are constants (typical values are $c_{1}=10^{-4}$ and $c_{2}=0.9$ ) [NW06, chapter 3]. Since $B_{k}^{-1}$ is positive definite, $\operatorname{tr}\left(G_{k}^{T} V_{k}\right)=\operatorname{tr}\left(G_{k}^{T} B_{k}^{-1} G_{k}\right)<0$; this guarantees the descent condition, i.e., $E\left(X_{k+1}\right)<E\left(X_{k}\right)$. There are many possible ways to find a direction satisfying these conditions; for example, the line search described in [NW06, §3.5] may be used. Typically, the step length $t_{k}=1$ is tried first. After a few iterations, $t_{k}=1$ is usually accepted, which makes the line search inexpensive in practice.

Finally, we note that unlike $G_{k}$, the search direction $V_{k}$ is not necessarily a tangent to $\mathcal{X}$ at $X_{k}$. While algorithms similar to ours require the search directions to be tangents (e.g., [HAG17; HHY18]), we find that this is unnecessary in practice.

\subsubsection{Projection onto constraints}

The next iterate $X_{k+1}$ is obtained by projecting $X_{k+1 / 2}=X_{k}+t_{k} V_{k}$ onto the constraint set $\mathcal{X}$, i.e.,

$$
X_{k+1}=\Pi_{\mathcal{X}}\left(X_{k+1 / 2}\right) .
$$

We will see below that the projections onto $\mathcal{C}$ and $\mathcal{A}$ are unnecessary, since $X_{k+1 / 2} \in$ $\mathcal{X}$ when $\mathcal{X}=\mathcal{C}$ or $\mathcal{X}=\mathcal{A}$. We will then describe how to efficiently compute the projection $\Pi_{\mathcal{S}}$.

Centered embeddings. As long as the first iterate $X_{0}$ satisfies $X_{0}^{T} \mathbf{1}=0$, then the intermediate iterates $X_{k+1 / 2}$ also satisfy $X_{k+1 / 2}^{T} \mathbf{1}=0$. To see this, first note that if $X^{T} \mathbf{1}=0$, then $\nabla E(X)^{T} \mathbf{1}=0$; this can be seen from the expression (5.1) for the gradient. Since $G=\nabla E(X)$ for unconstrained embeddings, if $X_{k}$ is centered, from (6.3) it follows that $X_{k+1 / 2} \in \mathcal{C}$. For this reason, for the constraint $X \in \mathcal{C}$, we can simply take

$$
X_{k+1}=X_{k+1 / 2}
$$


Anchored embeddings. As was the case for centered embeddings, provided that $X_{k} \in \mathcal{A}, X_{k+1 / 2} \in \mathcal{A}$ as well, so the projection is unnecessary. This is immediate from the expression (5.7) for the projected gradient $G$, which is simply the gradient with rows corresponding to the anchors replaced with zero.

Standardized embeddings. A projection of $Z \in \mathbf{R}^{n \times m}$ onto $\mathcal{S}$ is any $X \in \mathcal{S}$ that minimizes $\|X-Z\|_{F}^{2}$. (Since $\mathcal{S}$ is nonconvex, the projection can be non-unique; a simple example is $Z=0$, where any $X \in \mathcal{S}$ is a projection.) In the sequel, we will only need to compute a projection for $Z$ satisfying $Z^{T} \mathbf{1}=0$ and $\operatorname{rank} Z=m$. For such $Z$, the projection onto $\mathcal{S}$ is unique and can be computed from the singular value decomposition (SVD), as follows.

Let $Z=U \Sigma V^{T}$ denote the SVD of $Z$, with $U \in \mathbf{R}^{n \times m}, U^{T} U=I, \Sigma \in \mathbf{R}^{m \times m}$ diagonal with nonnegative entries, and $V \in \mathbf{R}^{m \times m}, V^{T} V=I$. The projection is given by

$$
\Pi_{\mathcal{S}}(Z)=\sqrt{n} U V^{T} .
$$

To see this, we first observe that the matrix $\Pi_{\mathcal{S}}(Z)$ is the projection of $Z$ onto the set $\left\{X \in \mathbf{R}^{n \times m} \mid(1 / n) X^{T} X=I\right\}$; this fact is well known (see, e.g., [FH55], $[$ Hig89, §4] or [AM12, §3]), and it is related to the orthogonal Procrustes problem [Sch66]. To show that $\Pi_{\mathcal{S}}(Z)$ is in fact the projection onto $\mathcal{S}$, it suffices to show that $\Pi_{\mathcal{S}}(Z)^{T} \mathbf{1}=0$. From $Z^{T} \mathbf{1}=0$, it follows that the vector $\mathbf{1}$ is orthogonal to the range of $Z$. Because $\operatorname{rank} Z=m$, the $m$ columns of $U$ form a basis for the range of $Z$. This means $U^{T} \mathbf{1}=0$ and in particular $\Pi_{\mathcal{S}}(Z)^{T} \mathbf{1}=\sqrt{n} V U^{T} \mathbf{1}=0$. It is worth noting that the projection (6.5) is inexpensive to compute, costing only $O\left(\mathrm{~nm}^{2}\right)$ time; in most applications, $m \ll n$.

We now explain why just considering centered and full rank $Z$ is sufficient. Note from (5.10) that $G_{k}^{T} \mathbf{1}=0$, for $k=1,2, \ldots$; therefore, from (6.3), we see that $X_{k+1 / 2}$ is centered as long as the initial iterate is centered. Since the rank of a linear transformation is preserved under small perturbations and $\operatorname{rank} X=m$ for any $X \in \mathcal{S}$, the intermediate iterate $X_{k+1 / 2}$ will be full rank provided that the step size $t_{k}$ is not too large.

From (6.5), it follows that Frobenius distance of $Z$ to $\mathcal{S}$ is given by

$$
\operatorname{dist}(Z, \mathcal{S})=\|Z-\Pi(Z)\|_{F}=\left(\sum_{i=1}^{m}\left(\sigma_{i}-\sqrt{n}\right)^{2}\right)^{1 / 2},
$$

where $\sigma_{i}$ are the diagonal entries of $\Sigma$, i.e., the singular values of $Z$. 


\subsubsection{Algorithm summary}

Algorithm 1 below summarizes our projected L-BFGS method.

Algorithm 1 Projected L-BFGS METHOD

given an initial point $X_{0} \in \mathcal{X}$, maximum iterations $K$, memory size $M$, tolerance $\epsilon>0$.

for $k=0, \ldots, K$

1. Projected gradient. Compute projected gradient $G_{k}=\Pi_{\mathcal{T}_{X_{k}}}\left(\nabla E\left(X_{k}\right)\right)$.

2. Stopping criterion. Quit if $\left\|G_{k}\right\|_{F} \leq \epsilon$.

3. Search direction. Compute the quasi-Newton search direction $V_{k}$, as in (6.1).

4. Line search. Find step length $t_{k}$ satisfying the modified Wolfe conditions (6.4).

5. Update. $X_{k+1}:=\Pi_{\mathcal{X}}\left(X_{k}+t_{k} V_{k}\right)$.

Our method is nearly parameter-free, except for the memory size $M$, which parametrizes the computation of $V_{k}$ in step 3, and possibly some parameters in the particular line search algorithm used. We have empirically found that a small $M$, specifically $M=10$, works well across a wide variety of problems, and that the line search parameters have very little effect. Additionally, the algorithm appears to be fairly robust to the choice of the initial iterate $X_{0}$, which may be chosen randomly or by a heuristic, such as initializing at a spectral embedding (projected onto $\mathcal{X}$ ).

Practical experience with our algorithm suggests that the use of projected gradients $G_{k}$, instead of the gradients $\nabla E\left(X_{k}\right)$, is important. In our experiments, our algorithm substantially decreases the time (and iterations) to convergence compared to standard gradient and L-BFGS implementations equipped with only $\Pi_{\mathcal{X}}$ (but not $\Pi_{\mathcal{T}_{X}}$ ). We show an example of this in $\S 7.1$, figure 7.2 .

Convergence. The MDE problem is in general nonconvex, so we cannot guarantee that algorithm 1 will converge to a global solution. Nonetheless, we have found that it reliably finds good embeddings in practice when applied to the constraint sets $\mathcal{C}$, $\mathcal{A}$, and $\mathcal{S}$. When we have applied the method to quadratic MDE problems, it has always found a global solution.

There is a large body of work studying optimization with orthogonality constraints (e.g., over the Stiefel manifold), which are closely related to the standardization constraint. See, for example, [EAS98; Man02; AMS09; AM12; JD15; HGA15; 
HAG18; $\mathrm{Hu}^{+} 19$; $\left.\mathrm{Che}^{+} 20\right]$. In particular, methods similar to algorithm 1 have been shown to converge to stationary points of nonconvex functions, when certain regularity conditions are satisfied [Ji07; RW12; HGA15; HAG18].

Computational complexity. Each iteration of algorithm 1 takes $O(m p+n m M)+$ $O\left(M^{3}\right)$ work, plus any additional work required to compute the projected gradient and the projection onto $\mathcal{X}$. Computing the average distortion and its gradient contributes $O(m p)$ work, and computing the search direction contributes $O(n m M)+$ $O\left(M^{3}\right)$. The overhead due to the projections is typically negligible; indeed, the overhead is zero for centered and anchored embeddings, and $O\left(n m^{2}\right)$ for standardized embeddings.

In most applications, the embedding dimension $m$ is much smaller than $n$ and $p$. Indeed, when an MDE problem is solved to produce a visualization, $m$ is just 2 or 3 ; when it is used to compute features, it is usually on the order of 100. In these common cases, the cost of each iteration is dominated by the cost of computing the average distortion and its gradient, which scales linearly in the number of pairs $p$. Suppressing the dependence on $m$ and $M$ (which we can treat as the constant 10), the cost per iteration is just $O(p)$. For some special distortion functions, this cost can be made as small as $O(n \log n)$ or $O(n)$, even when $p=n(n-1) / 2$, using fast multipole methods [GR87; BG97].

\subsection{A stochastic proximal algorithm}

Building on the projected L-BFGS method described in the previous section, here we describe a simple scheme to approximately solve MDE problems with a number of distortion functions $p$ too large to handle all at once. It can be considered an instance of the stochastic proximal iteration method (see, e.g., [RB14; AD19]), which uses much larger batch sizes than are typically used in stochastic optimization methods. (Stochastic proximal iteration is itself simply a stochastic version of the classical proximal point algorithm [Mar70; Roc76].) In each iteration, or round, we randomly choose a subset of $p^{\text {batch }}$ of the original $p$ distortion functions and (after the first round) approximately solve a slightly modified MDE problem.

In the first round, we approximately solve the MDE problem corresponding to the sampled distortion functions using algorithm 1, and we denote the resulting embedding as $X^{0}$. (We used superscripts here to denote rounds, to distinguish them from $X_{k}$, which are iterates in the methods described above.) In round $k$, $k=1,2, \ldots$, we randomly choose a set of $p^{\text {batch }}$ distortion functions, which gives us 
average distortion $E^{k}$, and approximately solve the MDE problem with an additional proximal term added to the objective, i.e.

$$
E^{k}(X)+\frac{c k}{2}\left\|X-X^{k-1}\right\|_{F}^{2}
$$

where $c$ is a hyper-parameter (we discuss its selection below). We denote the new embedding as $X^{k}$. The second term in the objective, referred to as a proximal term [PB14], encourages the next embedding to be close to the one found in the previous round. In computing $X^{k}$ in round $k$, we can warm-start the iterative methods described above by initializing $X$ as $X^{k-1}$. This can result in fewer iterations required to compute $X^{k}$.

When the objective and constraints are convex and satisfy some additional conditions, this algorithm can be proved to converge to a solution of the original problem. However, in an MDE problem, the objective is generally not convex, and for the standardized problem, nor is the constraint. Nevertheless we have found this method to reliably find good embeddings for problems with more edges than can be directly handled in one solve. The algorithm is outlined below.

Algorithm 2 Stochastic Method For MDE PROBlems With LARGE $p$. given a batch size $p^{\text {batch }}<p$, iterations $K$.

for $k=0, \ldots, K$

1. Select a batch of edges. Randomly select a batch of $p^{\text {batch }}$ distortion functions, which defines average distortion $E^{k}$.

2. First iteration. If $k=0$, solve the MDE problem with objective $E^{k}$ to get embedding $X^{0}$.

3. Subsequent iterations. If $k>0$, solve the MDE problem using the batch, with additional objective term as in (6.6), to get $X^{k}$.

Hyper-parameter selection. The empirical convergence rate of the algorithm is sensitive to the choice of hyper-parameter $c$. If $c$ is too big, we place too much trust in the iterate $X^{0}$ obtained by the first round, and the algorithm can make slow progress. If $c$ is too small, the algorithm oscillates between minimum-distortion embeddings for each batch, without converging to a suitable embedding for all $p$ distortion functions. For this reason, the selection of $c$ is crucial. 
The hyper-parameter can be chosen in many ways, including manual experimentation. Here we describe a specific method for automatically choosing a value of $c$, based on properties of the MDE problem being solved. We find this method works well in practice. The method chooses

$$
c=\frac{\operatorname{tr}\left(\nabla^{2} E^{0}\left(X^{0}\right)\right)}{\lambda n m}
$$

where $\lambda \in \mathbf{R}$ is a parameter (we find that the choice $\lambda=10$ works well). This choice of $c$ can be interpreted as using a diagonal approximation of the Hessian of $E^{0}$ at $X^{0}$, i.e.,

$$
\nabla^{2} E^{0}\left(X^{0}\right) \approx c I
$$

In our implementation, instead of computing the exact Hessian $\nabla^{2} E^{0}\left(X^{0}\right)$, which can be intractable depending on the size of $n$ and $m$, we approximate it using randomized linear algebra and Hessian-vector products. We specifically use the Hutch++ algorithm $\left[\mathrm{Mey}^{+} 20\right]$, a recent method based on the classical Hutchinson estimator [Hut89]. This algorithm requires a small number $q$ of Hessian-vector products, and additionally $O\left(m q^{2}\right)$ time to compute a $\mathrm{QR}$ decomposition of an $m \times q / 3$ matrix. Using modern systems for automatic differentiation, these Hessian-vector products can be computed efficiently, without storing the Hessian matrix in memory. For our application, $q=10$ suffices, making the cost of computing $c$ negligible. 


\section{Chapter 7}

\section{Numerical Examples}

In this chapter we present numerical results for several MDE problems. The examples are synthetic, meant only to illustrate the performance and scaling of our algorithms. The numerical results in this chapter were produced using a custom implementation of our solution algorithms. We first present the numerical examples; the implementation, which we have packaged inside a high-level Python library for specifying and solving MDE problems, is presented in $\S 7.4$.

In the first set of examples we apply the projected quasi-Newton algorithm (algorithm 1) to quadratic MDE problems. Using a specialized solver for eigenproblems, which can solve quadratic MDE problems globally, we verify that our method finds a global solution in each example. We also compare our algorithm to standard methods for smooth constrained optimization, which appear to be much less effective. The problems in this section are of small to medium size, with no more than one million items and ten million edges.

Next, in $\S 7.2$, we apply algorithm 1 to small and medium-size (non-quadratic) MDE problems derived from weights, with constraint sets $\mathcal{C}, \mathcal{A}$, and $\mathcal{S}$. We will see that the overhead incurred in handling these constraints is negligible.

In $\S 7.3$ we apply the stochastic proximal method (algorithm 2) to two MDE problems, one small and one very large. We verify that the method finds nearly optimal embeddings for the small problem, makes progress on the very large one, and is robust across different initializations for both.

Experiment setup. All examples were solved with the default parameters of our implementation. In particular, we used a memory size of 10, and terminated the projected quasi-Newton algorithm when the residual norm fell below $10^{-5}$. Experiments were run on a computer with an Intel i7-6700K CPU (which has $8 \mathrm{MB}$ of cache and 
four physical cores, eight virtual, clocked at $4 \mathrm{GHz}$ ), an NVIDIA GeForce GTX 1070 GPU (which has $8 \mathrm{~GB}$ of memory and 1920 cores at $1.5 \mathrm{GHz}$ ), and $64 \mathrm{~GB}$ of RAM.

\subsection{Quadratic MDE problems}

In this first numerical example we solve quadratic MDE problems with weights $w_{i j}=$ 1 for $(i, j) \in \mathcal{E}$. (Recall that a quadratic MDE problem, studied in chapter 3, has distortion functions $f_{i j}\left(d_{i j}\right)=w_{i j} d_{i j}^{2}$, and the constraint $X \in \mathcal{S}$.) The edges are chosen uniformly at random by sampling $p=|\mathcal{E}|$ unique pairs of integers between 1 and $n(n-1) / 2$, and converting each integer to a pair $(i, j)(1 \leq i<j \leq n)$ via a simple bijection. We solve two problem instances, a medium size one with dimensions

$$
n=10^{5}, \quad p=10^{6}, \quad m=2
$$

and a large one with dimensions

$$
n=10^{6}, \quad p=10^{7}, \quad m=2 .
$$

The medium size problem is solved on a CPU, and the large one is solved on a GPU.

Figure 7.1 plots the residual norm $\left\|G_{k}\right\|_{F}$ of the projected L-BFGS method (algorithm 1) when applied to these problems, against both elapsed seconds and iterations. On a CPU, it takes 55 iterations and 3 seconds to reach a residual norm of $10^{-5}$ on the first problem, over which the average distortion is decreased from an initial value of 4.00 to 0.931 . On a GPU, it takes 33 iterations and 4 seconds to reach the same tolerance on the second problem, over which the average distortion is decreased from 4.00 to 0.470 .

These quadratic MDE problems can be solved globally as eigenvalue problems, as described in chapter 3. We solve the two problem instances using LOBPCG, a specialized algorithm for large-scale eigenproblems [Kny01] We specifically used scipy.sparse.linalg.lobpcg, initialized with a randomly selected standardized matrix, restricted to search in the orthogonal complement of the ones vector, and limited to a maximum iteration count of 40 . For the medium size problem, LOBPCG obtained a solution with average distortion 0.932 in 2 seconds; for the large problem, it gives a solution with average distortion of 0.469 in 17 seconds. We conclude that our algorithm found global solutions for both problem instances. (We have not encountered a quadratic MDE problem for which our method does not find a global solution.) We also note that the compute time of our method is comparable to, or better than, more specialized methods based on eigendecomposition. 

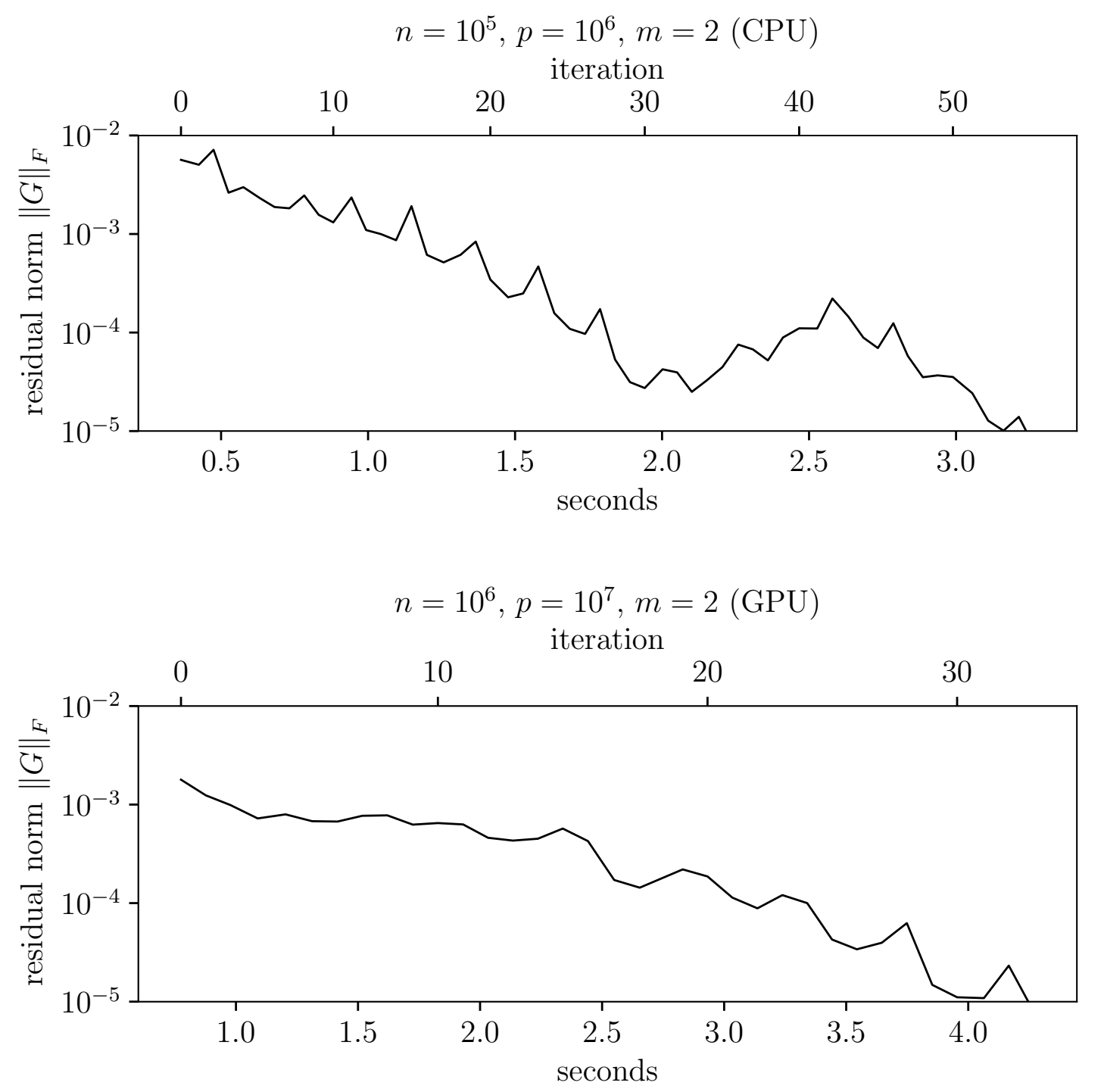

Figure 7.1: Residual norm versus iterations (top horizontal axis) and time (bottom horizontal axis) for the projected L-BFGS algorithm. Top. Medium size problem instance. Bottom. Large problem instance. 


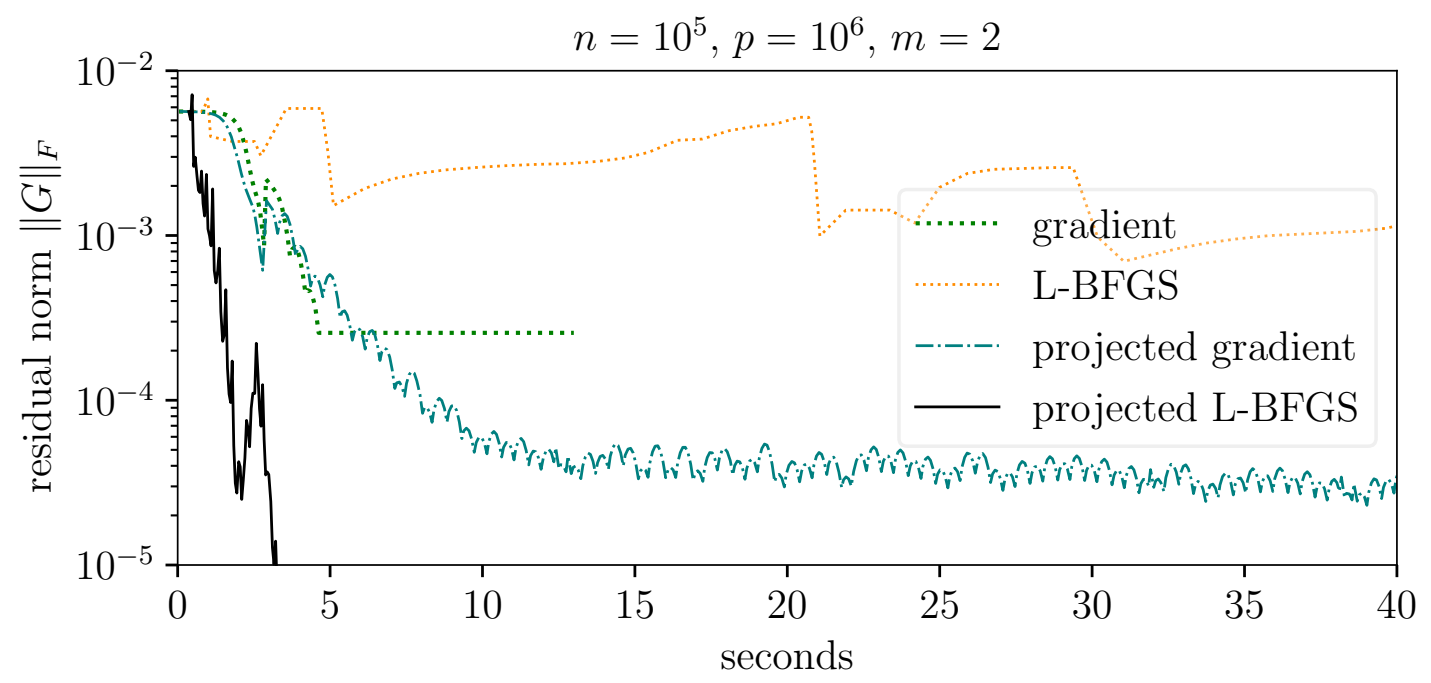

Figure 7.2: Residual norm versus seconds for our algorithm (projected-LBFGS) and other algorithms, on a medium size quadratic problem instance solved on CPU.

\subsubsection{Comparison to other methods}

The projected L-BFGS tends to converge to approximate solutions much faster than simpler gradient-based methods, such as standard gradient descent, which performs the update $X_{k+1}=\Pi_{\mathcal{X}}\left(X_{k}-t_{k} \nabla E\left(X_{k}\right)\right)$, and projected gradient descent, which performs the update $X_{k+1}=\Pi_{\mathcal{X}}\left(X_{k}-t_{k} G_{k}\right)$. Similarly, our method is much more effective than a standard L-BFGS method that only projects iterates onto the constraint set (but does not project the gradients).

To compare these methods, we solved the medium size quadratic problem instance on a CPU using each method, logging the residual norm versus elapsed seconds. (We used a standard backtracking-Armijo linesearch for the gradient methods.) Figure 7.2 shows the results. The gradient method fails to decrease the residual norm below roughly $3 \times 10^{-4}$, getting stuck after 66 iterations. The projected gradient method decreases the residual norm to just below $10^{-4}$ after about 8 seconds, but makes very slow progress thereafter. The standard L-BFGS method performs the worst, taking nearly 30 seconds to reach a residual norm of $10^{-3}$. The projected L-BFGS method finds a global solution, with $10^{-5}$ residual norm, in 3 seconds. 


\begin{tabular}{|c|c|c|c|c|c|c|}
\hline \multicolumn{3}{|c|}{ Problem instance dimensions } & \multicolumn{2}{|c|}{ Embedding time $(\mathrm{s})$} & \multicolumn{2}{|c|}{ Objective values } \\
\hline$n$ & $p$ & $m$ & $\mathrm{CPU}$ & GPU & $E\left(X_{K}\right)$ & $E\left(X^{\star}\right)$ \\
\hline $10^{3}$ & $10^{4}$ & 2 & 0.1 & 0.4 & 1.432 & 1.432 \\
\hline $10^{3}$ & $10^{4}$ & 10 & 0.2 & 0.4 & 7.797 & 7.795 \\
\hline $10^{3}$ & $10^{4}$ & 100 & 0.8 & 1.5 & 104.5 & 104.5 \\
\hline $10^{4}$ & $10^{5}$ & 2 & 0.4 & 0.4 & 1.007 & 1.007 \\
\hline $10^{4}$ & $10^{5}$ & 10 & 0.7 & 0.4 & 6.066 & 6.065 \\
\hline $10^{4}$ & $10^{5}$ & 100 & 20.8 & 2.9 & 81.12 & 81.08 \\
\hline $10^{5}$ & $10^{6}$ & 2 & 2.5 & 0.6 & 0.935 & 0.931 \\
\hline $10^{5}$ & $10^{6}$ & 10 & 10.5 & 1.2 & 5.152 & 5.137 \\
\hline $10^{5}$ & $10^{6}$ & 100 & 334.7 & 15.8 & 63.61 & 63.51 \\
\hline
\end{tabular}

Table 7.1: Embedding time and objective values when solving quadratic problem instances via algorithm 1 , for $K=40$ iterations.

\subsubsection{Scaling}

To get some idea of how our implementation scales, we solved instances with a variety of dimensions $n, m$, and $p$. We terminated the algorithm after $K=40$ iterations for each solve, noted the solve time, and compared the average distortion $E\left(X_{K}\right)$ with the average distortion of a global solution $X^{\star}$ obtained using LOBPCG. The results are listed in table 7.1. We can see that the scaling of our method is roughly $O(m p)$, which agrees with our previous discussion on computational complexity in §6.1. Additionally, for sufficiently large problems, GPU-accelerated solves are over 10 times faster than solving on CPU. After just 40 iterations, our method is nearly optimal for each problem instance, with optimality gaps of 0.4 percent or less.

\subsection{Other MDE problems}

As a second example, we solve MDE problems involving both positive and negative weights. The edges are chosen in the same way as the previous example. We randomly partition $\mathcal{E}$ into $\mathcal{E}_{\text {sim }}$ and $\mathcal{E}_{\text {dis }}$, and choose weights $w_{i j}=+1$ for $(i, j) \in \mathcal{E}_{\text {sim }}$ and $w_{i j}=-1$ for $(i, j) \in \mathcal{E}_{\text {dis }}$.

We use distortion functions based on penalties, of the form (4.1). We choose the log-one-plus penalty (4.3) for the attractive penalty, and the logarithmic penalty (4.4) for the repulsive penalty. 
As before, we solve problems of two sizes, a medium size one with

$$
n=10^{5}, \quad p=10^{6}, \quad m=2
$$

and a large one with

$$
n=10^{6}, \quad p=10^{7}, \quad m=2 .
$$

We solve three medium problems and three large problems, with constraints $X \in \mathcal{C}$, $X \in \mathcal{A}$, and $X \in \mathcal{S}$. For the anchor constraints, a tenth of the items are made anchors, with associated values sampled from a standard normal distribution. The medium size problems are solved on a CPU, and the large problems are solved on a GPU.

Figure 7.3 plots the residual norm of the L-BFGS methods when applied to these problems, against both seconds elapsed and iterations; all problems are solved to a residual norm of $10^{-5}$. Note the embedding times for centered and anchored embeddings are comparable, and the per-iteration overhead of computing the projections associated with the standardization constraint is small. 

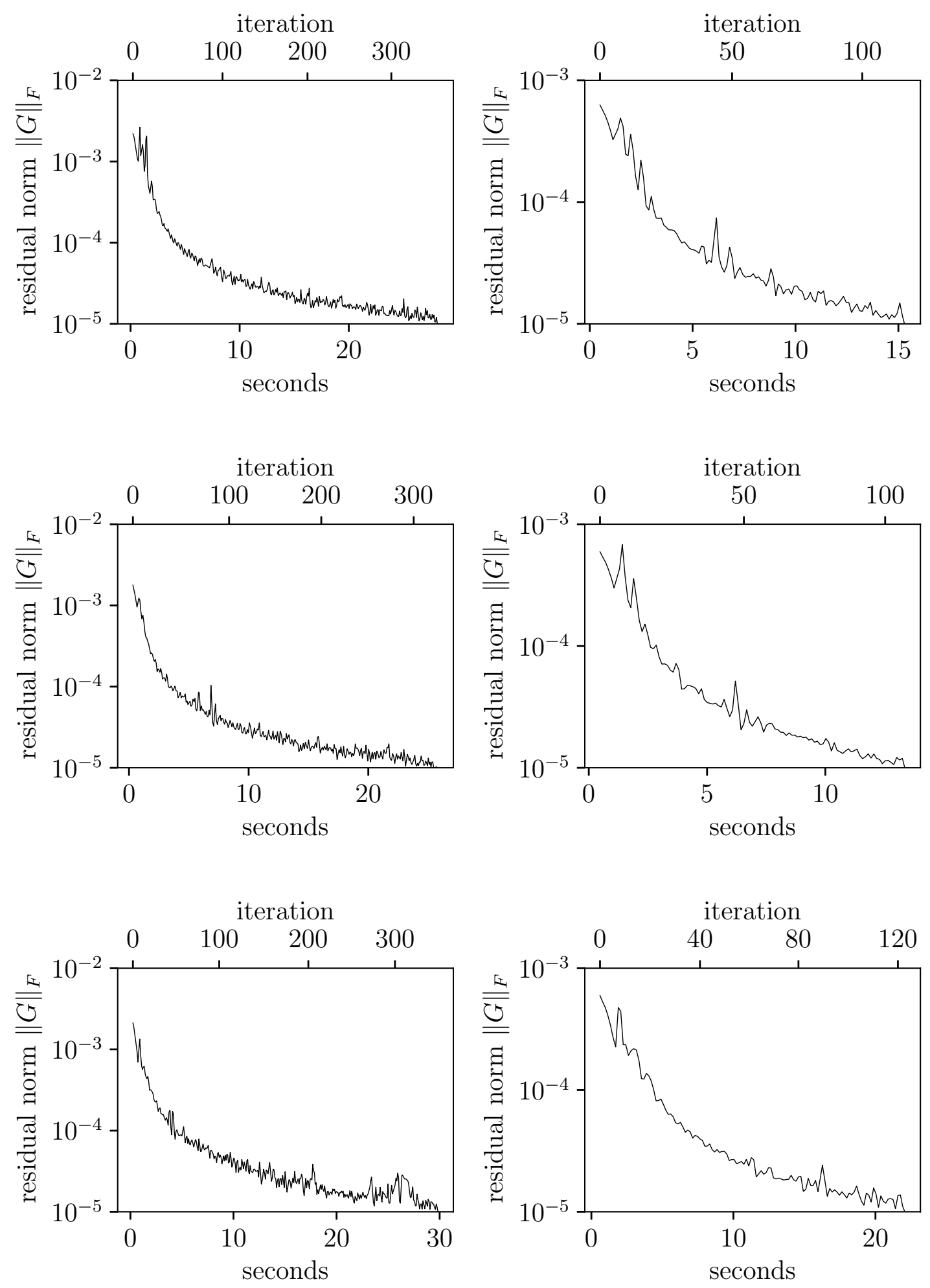

Figure 7.3: Residual norm versus iterations (top horizontal axis) and time (bottom horizontal axis) for the projected L-BFGS algorithm, applied to MDE problems derived from weights. Top. Centered. Middle. Anchored. Bottom. Standardized. Left. Medium problem instances (CPU). Right. Large problem instances (GPU). 


\subsection{A very large problem}

In this third set of examples we use the stochastic proximal method (algorithm 2) to approximately solve some quadratic MDE problems. To show that our method is at least reasonable, we first apply our method to a small quadratic MDE problem; we solve this MDE problem exactly, and compare the quality of the approximate solution, obtained by the stochastic method, to the global solution. Next we apply the stochastic method to a problem so large that we cannot compute a global solution, though we can evaluate its average distortion, which the stochastic method is able to steadily decrease.

In our experiments, we set the hyper-parameter $c$ as

$$
c \approx \frac{\operatorname{tr}\left(\nabla^{2} E^{0}\left(X^{0}\right)\right)}{10 n m},
$$

using a randomized method to approximate the Hessian trace, as described in §6.2.

A small problem. We apply our stochastic proximal method to a small quadratic MDE problem of size

$$
n=10^{3}, \quad p=10^{4}, \quad m=10,
$$

generated in the same fashion as the other quadratic problems. We approximately solve the same instance 100 times, using a different random initialization for each run. For these experiments, we use $p^{\text {batch }}=p / 10$, sample the batches by cycling through the edges in a fixed order, and run the method for 300 rounds. This means that we passed through the full set of edges 30 times.

Figure 7.4 shows the average distortion and residual norms versus rounds (the solid lines are the medians across all 100 runs, and the cone represents maximum and minimum values). The mean distortion of the final embedding was 7.89, the standard deviation was 0.03 , the max was 7.97 , and the min was 7.84 ; a global solution has an average distortion of 7.80. 

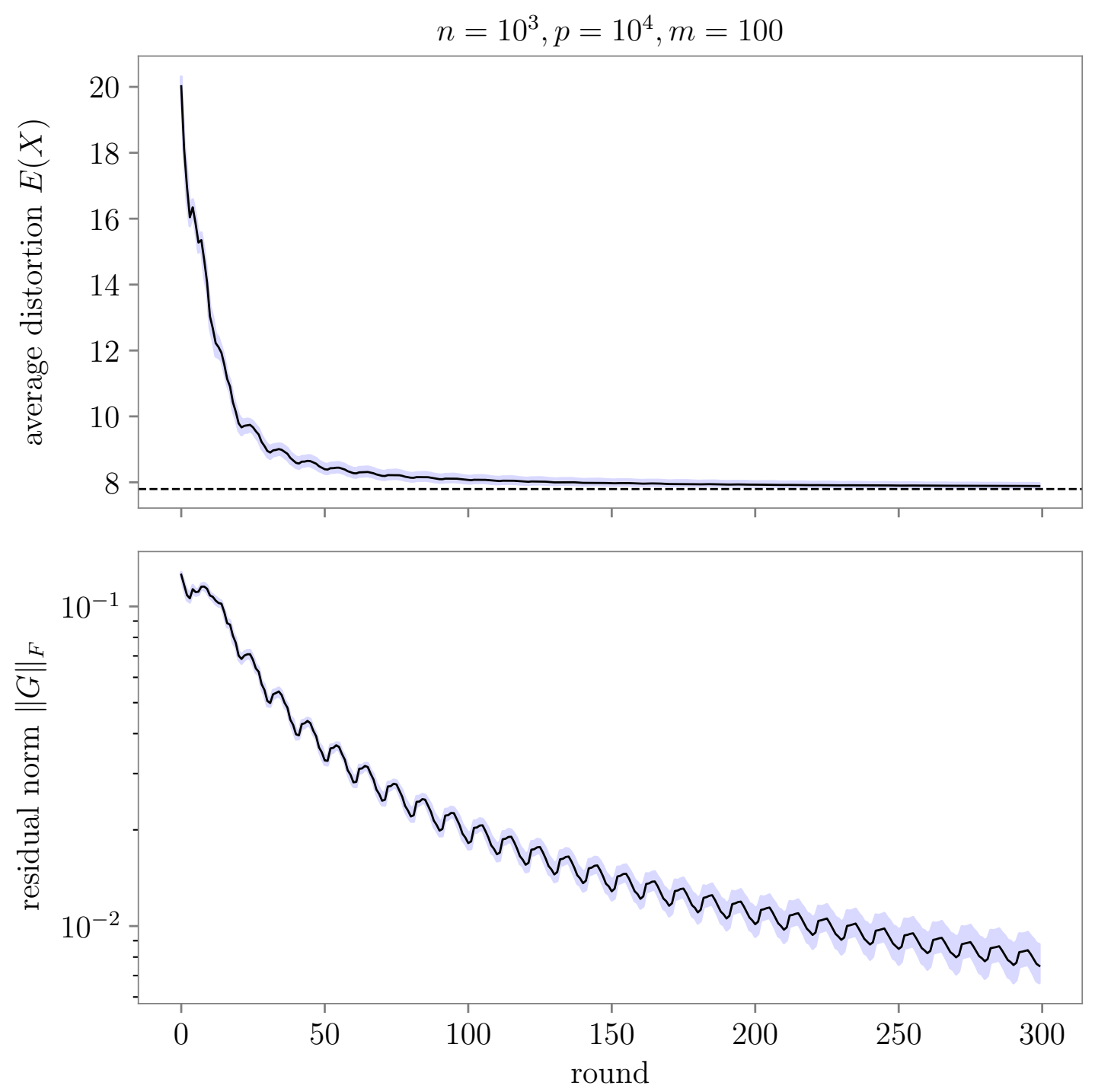

Figure 7.4: The stochastic method (algorithm 2) applied to a small quadratic MDE problem, with 100 different initializations. Top. Median average distortion versus rounds, optimal value plotted as a dashed horizontal line. Bottom. Median residual norm versus rounds. 
A very large problem. We approximately solve a very large quadratic MDE problem, with

$$
n=10^{5}, \quad p=10^{7}, \quad m=100 .
$$

This problem is too large to solve using off-the-shelf specialized solvers for eigenproblems such as scipy.sparse.linalg.lobpcg. It is also too large to fit on the GPU we use in our experiments, since storing the $p \times m$ matrix of differences alone takes roughly 4 GB of memory (and forming it can take up to 8 GB of memory). While this problem does fit in our workstation's 64 GB of RAM, evaluating the average distortion and its gradient takes 12 seconds, which makes running the full-batch projected quasi-Newton algorithm on CPU impractical.

We solved a specific problem instance, generated in the same way as the other quadratic MDE problems. We used $p^{\text {batch }}=p / 10$, sampled the batches by cycling through the edges in a fixed order, and ran the stochastic proximal iteration for 40 rounds. We ran 10 trials, each initialized at a randomly selected iterate. Figure 7.5 shows the median average distortion and residual norm at each round, across all $p$ pairs. On our GPU, the 40 rounds took about 10 minutes to complete. The mean average distortion after 40 rounds was 157.94 , the standard deviation was 0.14 , the maximum was 158.21, and the min was 157.71 . 

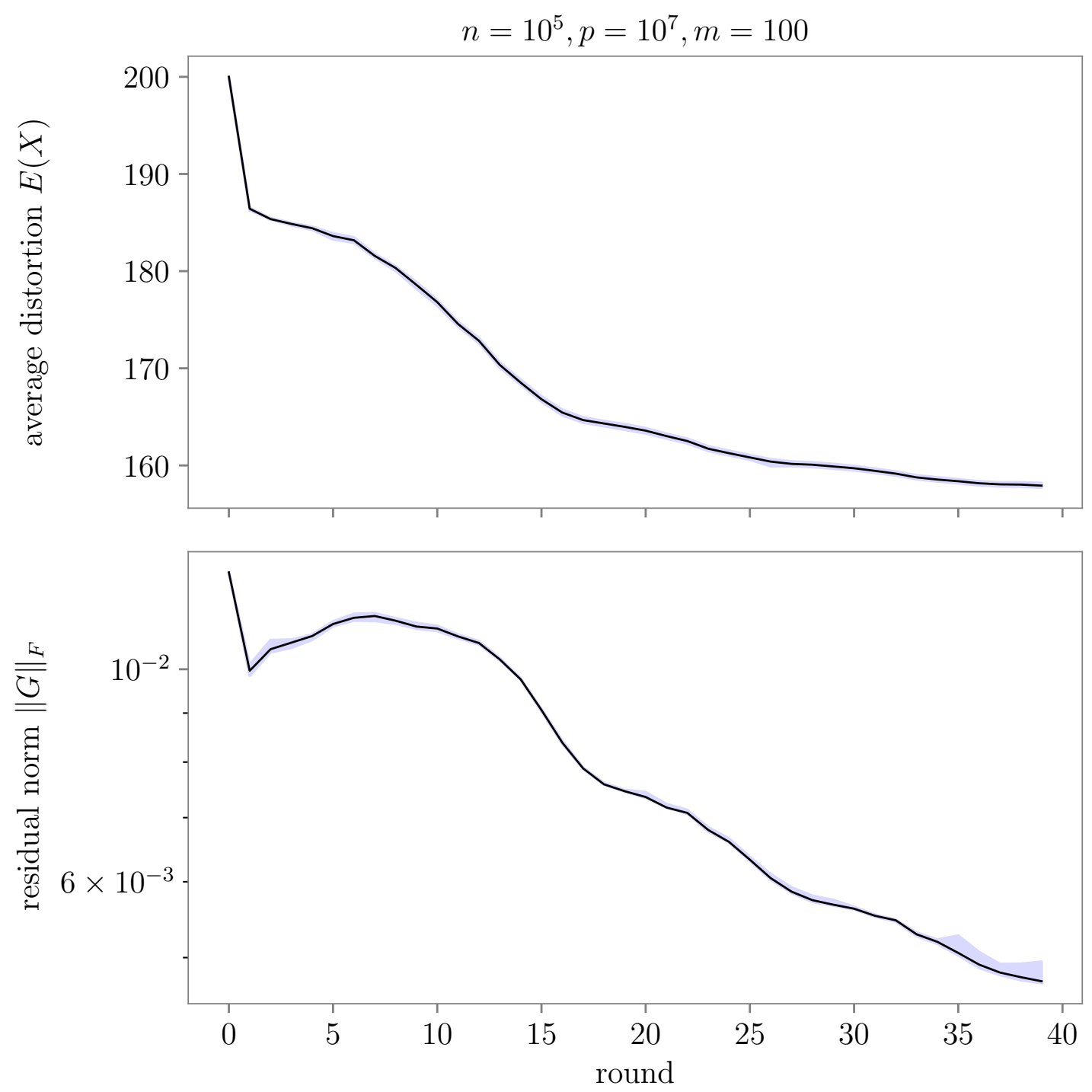

Figure 7.5: The stochastic method applied to a very large quadratic MDE problem, with 10 different initializations. 


\subsection{Implementation}

We have implemented our solution methods in PyMDE, an object-oriented Python package for specifying and approximately solving MDE problems. In addition, PyMDE provides higher-level interfaces for preprocessing data, visualizing embeddings, and other common tasks. PyMDE is designed to be simple to use, easily extensible, and performant.

Our software is open-source, and available at

$$
\text { https://github.com/cvxgrp/pymde. }
$$

\subsubsection{Design}

In this section we briefly describe the design of PyMDE.

Embedding. In our package, users instantiate an MDE object by specifying the number of items $n$, the embedding dimension $m$, the list of edges $\mathcal{E}$, the vector distortion function $f$, and the constraint set $(\mathcal{C}, \mathcal{A}$, or $\mathcal{S})$. Calling the embed method on this object runs the appropriate solution method and returns an embedding, represented as a dense matrix.

The distortion function may be selected from a library of functions, including the functions listed in chapter 4, but it can also be specified by the user. We use automatic differentiation (via PyTorch $\left[\mathrm{Pas}^{+} 19\right]$ ) to cheaply differentiate through distortion functions. For performance, we manually implement the gradient of the average distortion, using an efficient implementation of the analytical expression (5.1).

Extensibility. PyMDE can be easily extended, at no performance cost. Users can simply write custom distortion functions in PyTorch, without modifying the internals of our package. Likewise, users can implement custom constraint sets $\mathcal{X}$ by subclassing the Constraint class.

Performance. On modern CPUs, PyMDE can compute embeddings for hundreds of thousands of items and millions of edges in just a few seconds (if the embedding dimension is not too large). On a modest GPU, in the same amount of time, PyMDE can compute embeddings with millions of items and tens of millions of edges, as we have seen in the previous sections.

The most expensive operation in an iteration of algorithm 1 is evaluating the average distortion, specifically computing the differences $x_{i}-x_{j}$ for $(i, j) \in \mathcal{E}$. On 
a CPU, this can become a bottleneck if $p$ is greater than, say, $10^{6}$. A GPU can greatly accelerate this computation. For cases when $p$ is very large and a GPU is unavailable (or has insufficient memory), we provide an implementation of the stochastic proximal algorithm 2.

By default we use single-precision floating point numbers, since we typically do not need many significant figures in the fractional part of our embedding. To minimize numerical drift, for centered and standardized embeddings, we de-mean the iterate at the end of each iteration of algorithm 1.

\subsubsection{Examples}

Here we demonstrate PyMDE with basic code examples.

Hello, world. The below code block shows how to construct and solve a very simple MDE problem, using distortion functions derived from weights. This specific example embeds a complete graph on three vertices using quadratic distortion functions, similar to the simple graph layout examples from $\S 2.5$.

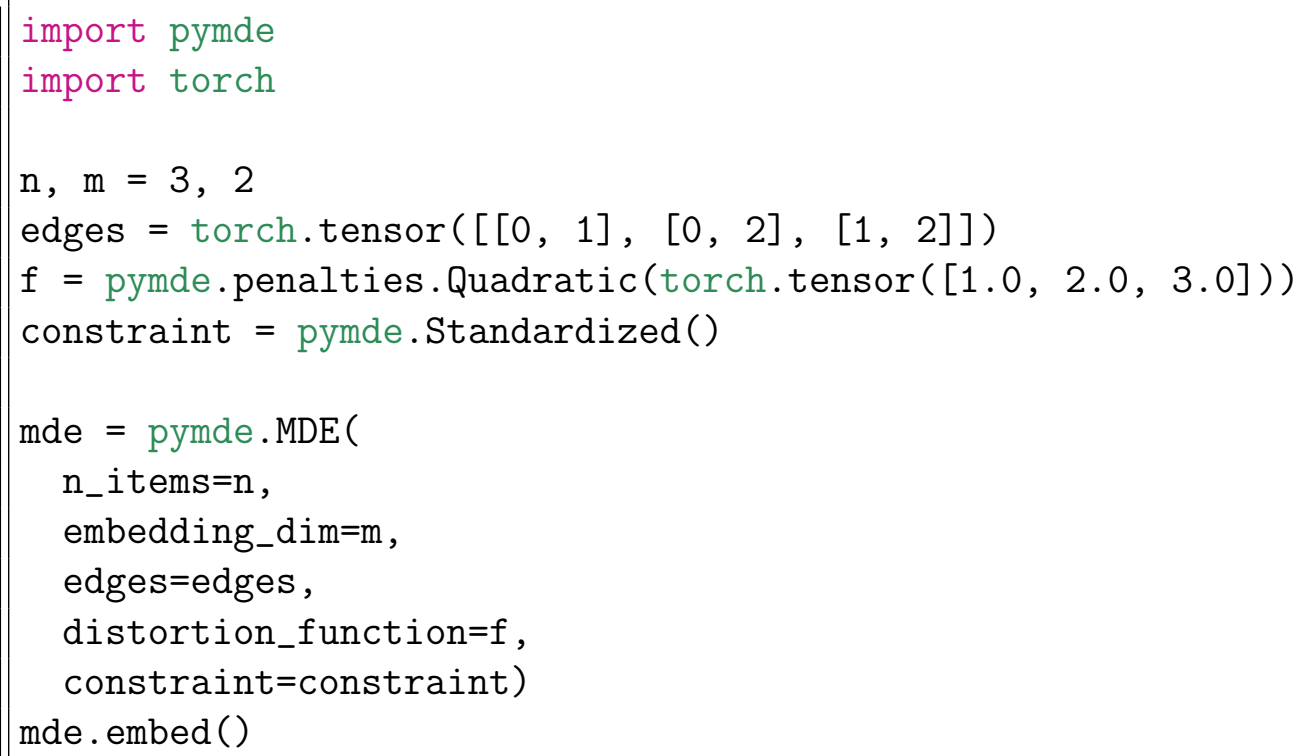

In this code block, after importing the necessary packages, we specify the number of items $n$ and the embedding dimension $m$. Then we construct the list edges of item pairs; in this case, there are just three edges, $(0,1),(0,2)$, and $(1,2)$. Next we construct the vector distortion function $\mathrm{f}$, a quadratic penalty with weight $w_{1}=1$ across 
the edge $(0,1), w_{2}=2$ across $(0,2)$, and $w_{3}=3$ across $(1,2)$. Then we create a standardization constraint. In the last two lines, the MDE problem is constructed and a minimum-distortion embedding is computed via the embed method. This method populates the attribute $\mathrm{X}$ on the MDE object with the final embedding. It also writes the distortion and the residual norm to the attributes value and residual_norm:

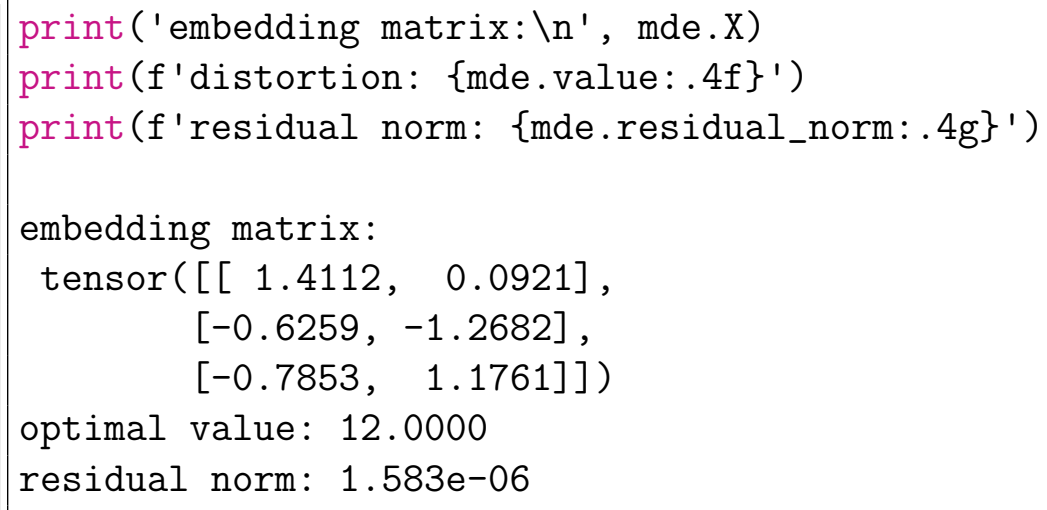

The MDE class has several other methods, such as average_distortion, which computes the average distortion of a candidate embedding, plot, which plots the embedding when the dimension is one, two, or three, and play, which generates a movie showing the intermediate iterates formed by the solution method. Additionally, the embed method takes a few optional arguments, through which (e.g.) an initial iterate, the memory size, iteration limit, and solver tolerance can be specified.

Distortion functions. PyMDE's library of distortion functions includes all the functions listed in $\S 4.1$ and $\S 4.2$, among others. Users simply construct a vector distortion function by passing weights or original deviations to the appropriate constructor. Every parameter appearing in a distortion function has a sensible default value (which the user can optionally override). PyMDE uses single-index notation for distortion functions, meaning the $k$ th component of the embedding distances corresponds to the $k$ th row of the list of edges supplied to the MDE constructor. For example, a quadratic penalty with $f_{k}\left(d_{k}\right)=w_{k} d_{k}^{2}$ can be constructed with pymde.penalties.Quadratic (weights), while a quadratic loss with $f_{k}\left(d_{k}\right)=$ $\left(d_{k}-\delta_{k}\right)^{2}$ can be constructed with pymde.losses.Quadratic (deviations).

For functions derived from both positive and negative weights, we provide a generic distortion function based on attractive and repulsive penalties, of the form (4.1). As an example, a distortion function with a log-one-plus attractive penalty and a logarithmic repulsive penalty can be constructed as 


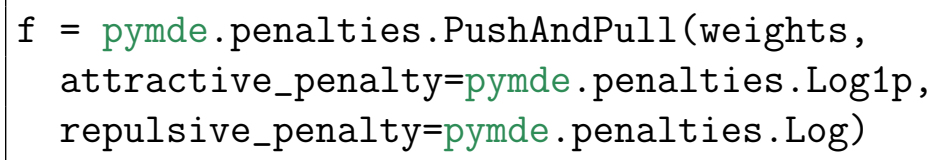

Users can implement their own distortion functions. These simply need be Python callables mapping the vector of embedding distances to the vector of distortions via PyTorch operations. For example, a quadratic penalty can be implemented as

def $f$ (distances):

return weights $*$ distances $* * 2$

and a quadratic loss can be implemented as

def $\mathrm{f}$ (distances):

return (deviations - distances) $* * 2$

(note the weights and deviations are captured by lexical closure). More generally, the distortion function may be any callable Python object, such as a torch.nn. Module.

Constraints. Each MDE object is parametrized by a constraint. PyMDE currently implements three constraint sets, which can be constructed using pymde. Centered(), pymde.Standardized(), and pymde.Anchored(anchors, values), where anchors is a torch.Tensor enumerating the anchors and values enumerates their values. Custom constraints can be implemented by subclassing the Constraint class and implementing the methods project_tspace and project.

Preprocessors. We have implemented all the preprocessors described in $\S 8.2$, in the module pymde.preprocess. These preprocessors can be used to transform original data and eventually obtain an MDE problem.

Several of these preprocessors take as input either a data matrix $Y$ with $n$ rows, with each row a (possibly high dimensional) vector representation of an item, or a sparse adjacency matrix $A \in \mathbf{R}^{n \times n}$ of a graph (wrapped in a pymde.Graph object), with $A_{i j}$ equal to an original deviation $\delta_{i j}$ between items $i$ and $j$. For example, the below code constructs a $k$-nearest neighborhood graph from a data matrix $Y$.

graph $=$ pymde.preprocess.k_nearest_neighbors $(\mathrm{Y}, \mathrm{k}=15)$ 
The same function can be used for a graph object, with adjacency matrix $A$.

graph $=$ pymde.preprocess.k_nearest_neighbors (pymde.Graph(A), k=15)

The associated edges and weights are attributes of the returned object.

edges, weights = graph.edges, graph.weights

We have additionally implemented a preprocessor for computing some or all of the graph distances (i.e., shortest-path lengths), given a graph. Our (custom) implementation is efficient and can exploit multiple CPU cores: the algorithm is implemented in $\mathrm{C}$, and we use multi-processing for parallelism. As an example, computing roughly 1 billion graph distances takes just 30 seconds using 6 CPUs.

Other features. PyMDE includes several other features, including a stochastic solve method, high-level interfaces for constructing MDE problems for preserving neighborhoods or distances, visualization tools and more. The full set of features, along with code examples, is presented in our online documentation at 


\section{Part III}

\section{Examples}




\section{Chapter 8}

\section{Images}

In this chapter we embed the well-known MNIST collection of images [LCB98], given by their grayscale vectors, into $\mathbf{R}^{2}$ or $\mathbf{R}^{3}$. Embeddings of images in general, into three or fewer dimensions, provide a natural user interface for browsing a large number of images; e.g., an interactive application might display the underlying image when the cursor is placed over an embedding vector. All embeddings in this chapter (and the subsequent ones) are computed using the experiment setup detailed in chapter 7 .

\subsection{Data}

The MNIST dataset consists of $n=70,000,28$-by-28 grayscale images of handwritten digits, represented as vectors in $\mathbf{R}^{784}$, with components between 0 and 255 . Each image is tagged with the digit it depicts. The digit is a held-out attribute, i.e., we do not use the digit in our embeddings, which are constructed using only the raw pixel data. We use the digit attribute to check whether the embeddings are sensible, as described in $\S 2.6$.

\subsection{Preprocessing}

We use distortion functions derived from weights. The set of similar pairs $\mathcal{E}_{\text {sim }}$ holds the edges of a $k$-nearest neighbor graph, using $k=15$; in determining neighbors, we use the Euclidean distance between image vectors. (The Euclidean distance is in general a poor global metric on images, but an adequate local one. Two images with large Euclidean distance might be similar e.g., if one is a translation or rotation of the other, but two images with small Euclidean distance are surely similar.) 
To compute the nearest neighbors efficiently, we use the approximate nearest neighbor algorithm from [DCL11], implemented in the Python library pynndescent [McI20a]. Computing the neighbors takes roughly 30 seconds. The resulting neighborhood graph is connected and has 774,746 edges. We sample uniformly at random an additional $\left|\mathcal{E}_{\text {sim }}\right|$ pairs not in $\mathcal{E}_{\text {sim }}$ to obtain the set $\mathcal{E}_{\text {dis }}$ of dissimilar image pairs. We assign $w_{i j}=+2$ if $i$ and $j$ are both neighbors of each other; $w_{i j}=+1$ if $i$ is a neighbor of $j$ but $j$ is not a neighbor of $i$ (or vice versa); and $w_{i j}=-1$ for $(i, j) \in \mathcal{E}_{\text {dis }}$.

\subsection{Embedding}

We consider two types of MDE problems: (quadratic) MDE problems based on the neighborhood graph $\mathcal{E}_{\text {sim }}$ (which has 774,746 edges), and MDE problems based on the graph $\mathcal{E}_{\text {sim }} \cup \mathcal{E}_{\text {dis }}$ (which has $1,549,492$ edges).

\subsubsection{Quadratic MDE problems}

We solve two quadratic MDE problems on the graph $\mathcal{E}_{\text {sim }}$ (i.e., MDE problems with quadratic penalties and a standardization constraint; the resulting embeddings can be interpreted as Laplacian embeddings, since the weights are nonnegative). The first problem has embedding dimension $m=2$ and the second has dimension $m=3$.

We solve these MDE problems using algorithm 1. Using PyMDE, embedding into $\mathbf{R}^{2}$ takes 1.8 seconds on our GPU and 3.8 seconds on our CPU; embedding into $\mathbf{R}^{3}$ takes 2.6 seconds on GPU and 7.5 seconds on CPU. The solution method terminates after 98 iterations when $m=2$ and after 179 iterations when $m=3$.

The embeddings are plotted in figures 8.1 and 8.2, with the embedding vectors colored by digit. In both cases, the same digits are clustered near each other in the embeddings. 


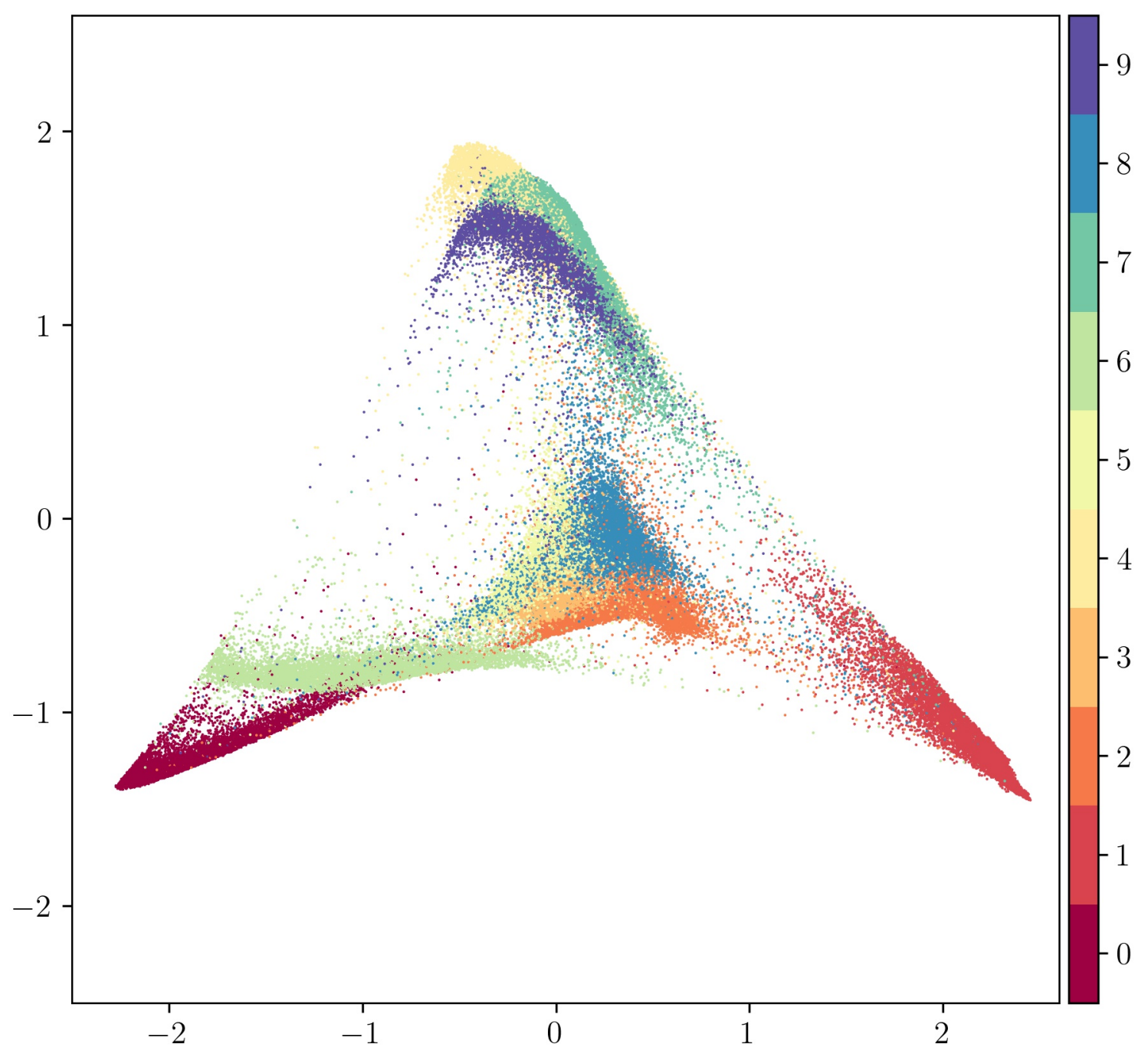

Figure 8.1: Embedding based on $\mathcal{E}_{\text {sim }}(m=2)$. An embedding of MNIST, obtained by solving a quadratic MDE problem derived from a neighborhood graph. 


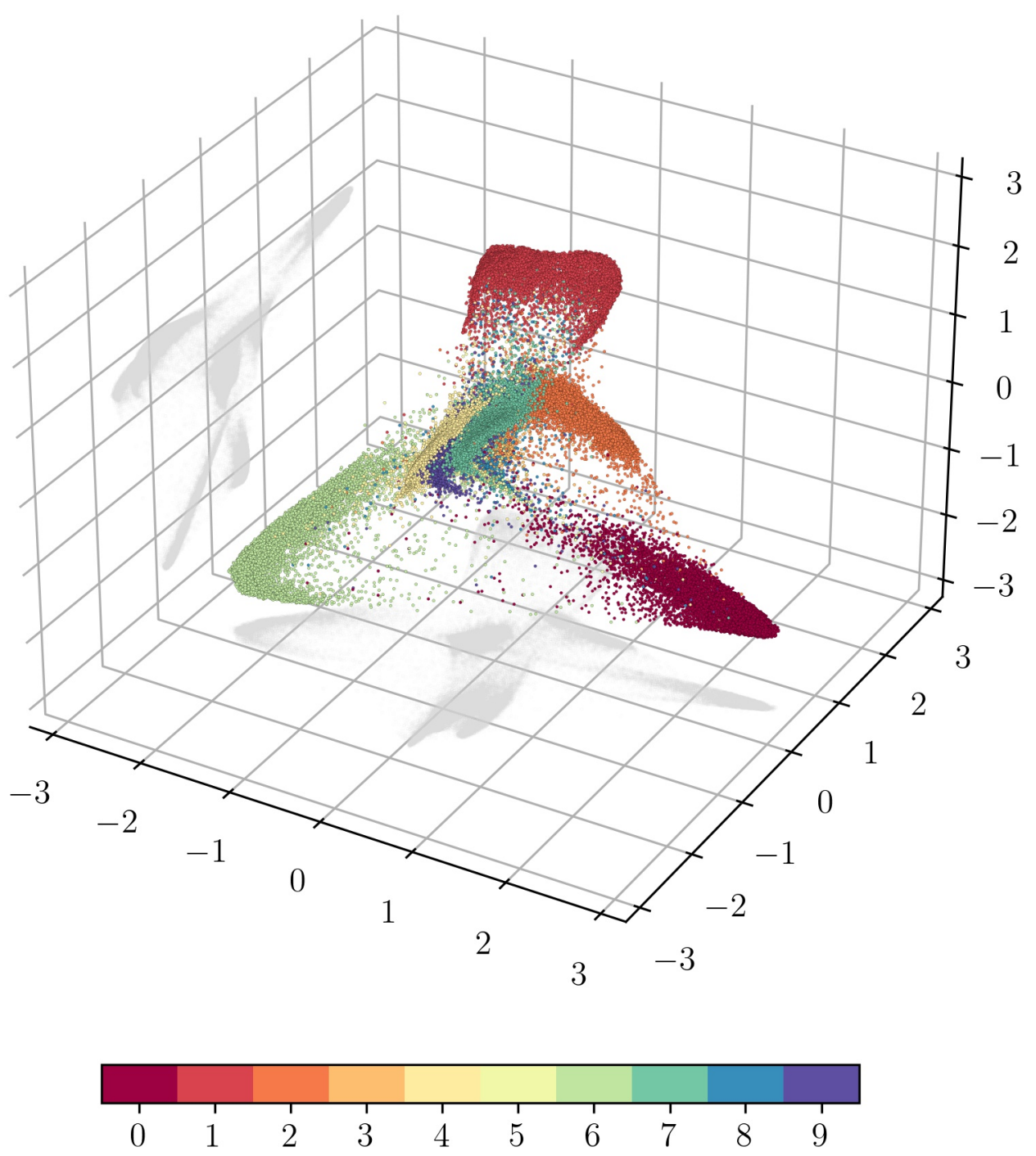

Figure 8.2: Embedding based on $\mathcal{E}_{\text {sim }}(m=3)$. An embedding of MNIST, obtained by solving a quadratic MDE problem derived from a neighborhood graph. 


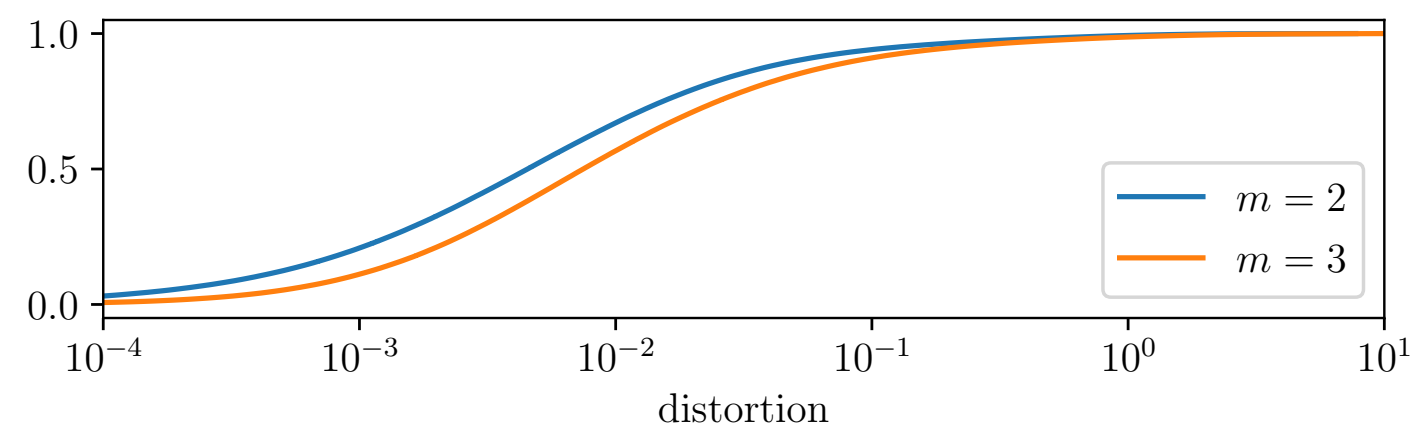

Figure 8.3: Distortions. CDF of distortions for the quadratic MDE problem.
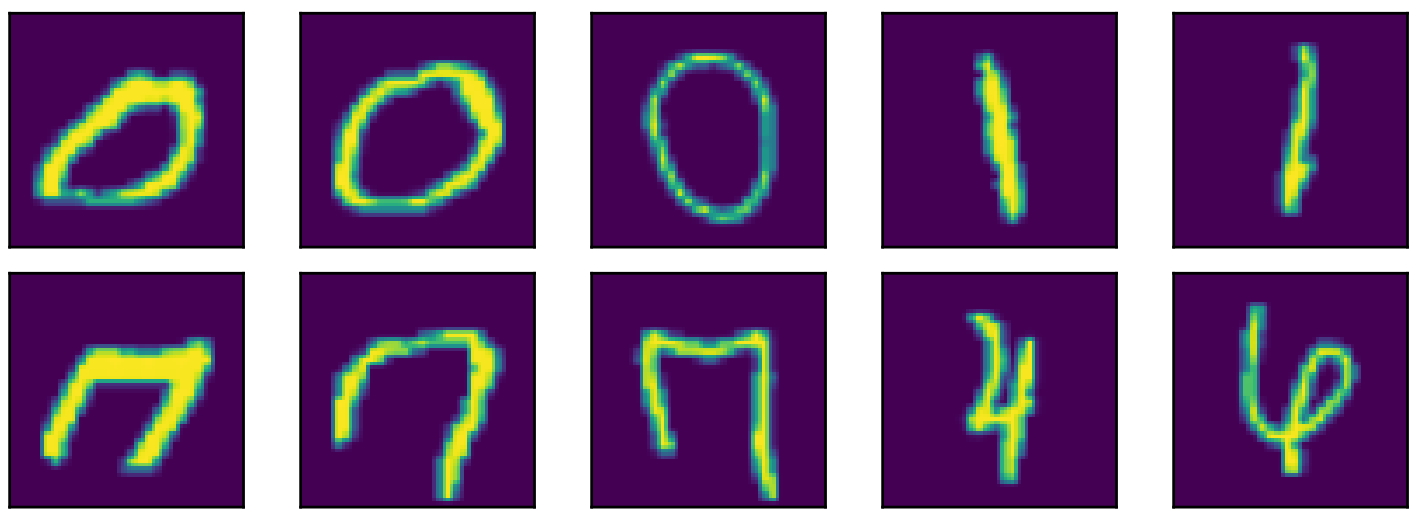

Figure 8.4: Outliers. Five pairs of images associated with item pairs with large distortion.

Outliers. The embeddings obtained by solving the quadratic MDE problem have low average distortion, but some pairs $(i, j)$ have much larger distortion $f_{i j}\left(d_{i j}\right)$ than others. The cumulative distribution functions (CDFs) of distortions are shown in figure 8.3. Figure 8.4 plots the images associated with the five pairs in $\mathcal{E}_{\text {sim }}$ that incurred the highest distortion, for the embedding into $\mathbf{R}^{2}$. Each column shows a pair of images. Some of these pairs do not appear to be very similar, e.g., a 1 is paired with a 4 . Other pairs include images that are nearly illegible. 


\subsubsection{Other embeddings}

Next we solve MDE problems with distortion functions derived from both positive and negative weights. In each MDE problem, the distortion functions are based on two penalty functions, as in (4.1): $p_{\mathrm{s}}$, which measures the distortion for pairs in $\mathcal{E}_{\text {sim }}$, and $p_{\mathrm{d}}$, which measures the distortion for pairs in $\mathcal{E}_{\text {dis }}$. All the MDE problems take $p_{\mathrm{d}}$ to be the logarithmic penalty (4.4), with hyper-parameter $\alpha=1$. For each problem, we initialize the solution method at the solution to the quadratic MDE problem from the previous section.

All embeddings plotted in the remainder of this chapter were aligned to the embedding in figure 8.1, via orthogonal transformations, as described in §2.4.5.

Standardized embeddings. The first problem is derived from the graph $\mathcal{E}=$ $\mathcal{E}_{\text {sim }} \cup \mathcal{E}_{\text {dis }}\left(\right.$ with $\left.\left|\mathcal{E}_{\text {sim }}\right|=\left|\mathcal{E}_{\text {dis }}\right|=774,776\right)$. We impose a standardization constraint and use the log-one-plus penalty (4.3) for $p_{\mathrm{s}}$, with hyperparameter $\alpha=1.5$.

The solution method ran for 177 iterations, taking 6 seconds on a GPU and 31 seconds on a CPU. Figure 8.5 shows an embedding obtained by solving this MDE problem. Notice that this embedding is somewhat similar to the quadratic one from figure 8.1, but with more separation between different classes of digits.

Varying the number of dissimilar pairs. We solve five additional MDE problems, using the same penalties and constraints as the previous one but varying the number of dissimilar pairs, to show how the embedding depends on the ratio $\left|\mathcal{E}_{\text {sim }}\right| /\left|\mathcal{E}_{\text {dis }}\right|$. The results are plotted in figure 8.6. When $\left|\mathcal{E}_{\text {dis }}\right|$ is small, items belonging to similar digits are more tightly clustered together; as $\left|\mathcal{E}_{\text {dis }}\right|$ grows larger, the embedding becomes more spread out.

Varying the weights for dissimilar pairs. Finally, we solve another five MDE problems of the same form, this time varying the magnitude of the weights $w_{i j}$ for $(i, j) \in \mathcal{E}_{\text {dis }}$ but keeping $\left|\mathcal{E}_{\text {dis }}\right|=\left|\mathcal{E}_{\text {sim }}\right|$ fixed. The embeddings are plotted in figure 8.7. Varying the weights (keeping $\left|\mathcal{E}_{\text {dis }}\right|$ fixed) has a similar effect to varying $\left|\mathcal{E}_{\text {dis }}\right|$ (keeping the weights fixed). 


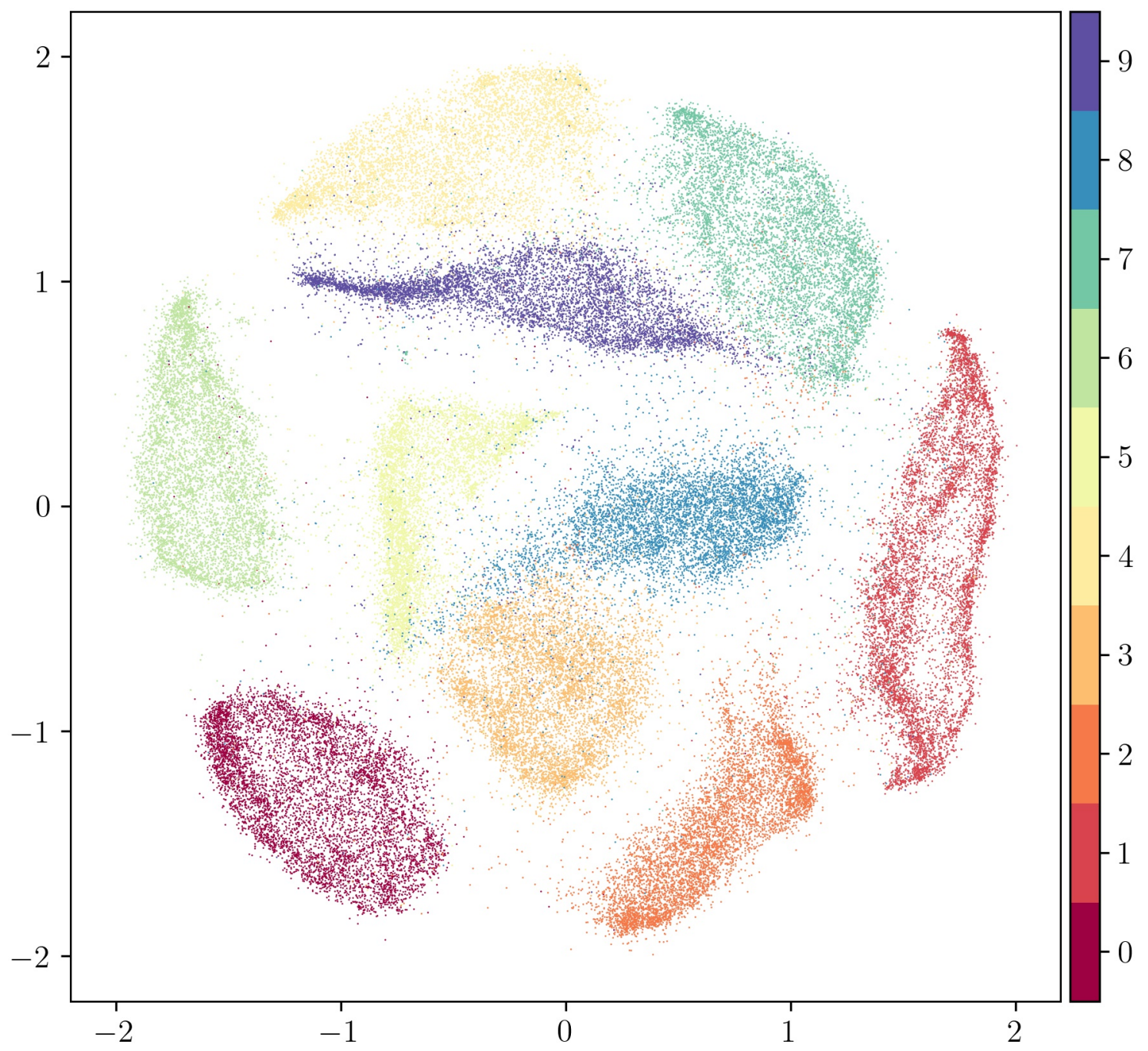

Figure 8.5: Embedding based on $\mathcal{E}_{\text {sim }} \cup \mathcal{E}_{\text {dis }}$. A standardized embedding of MNIST, based on the union of a neighborhood graph and a dissimilarity graph, with log-oneplus attractive penalty and logarithmic repulsive penalty. 

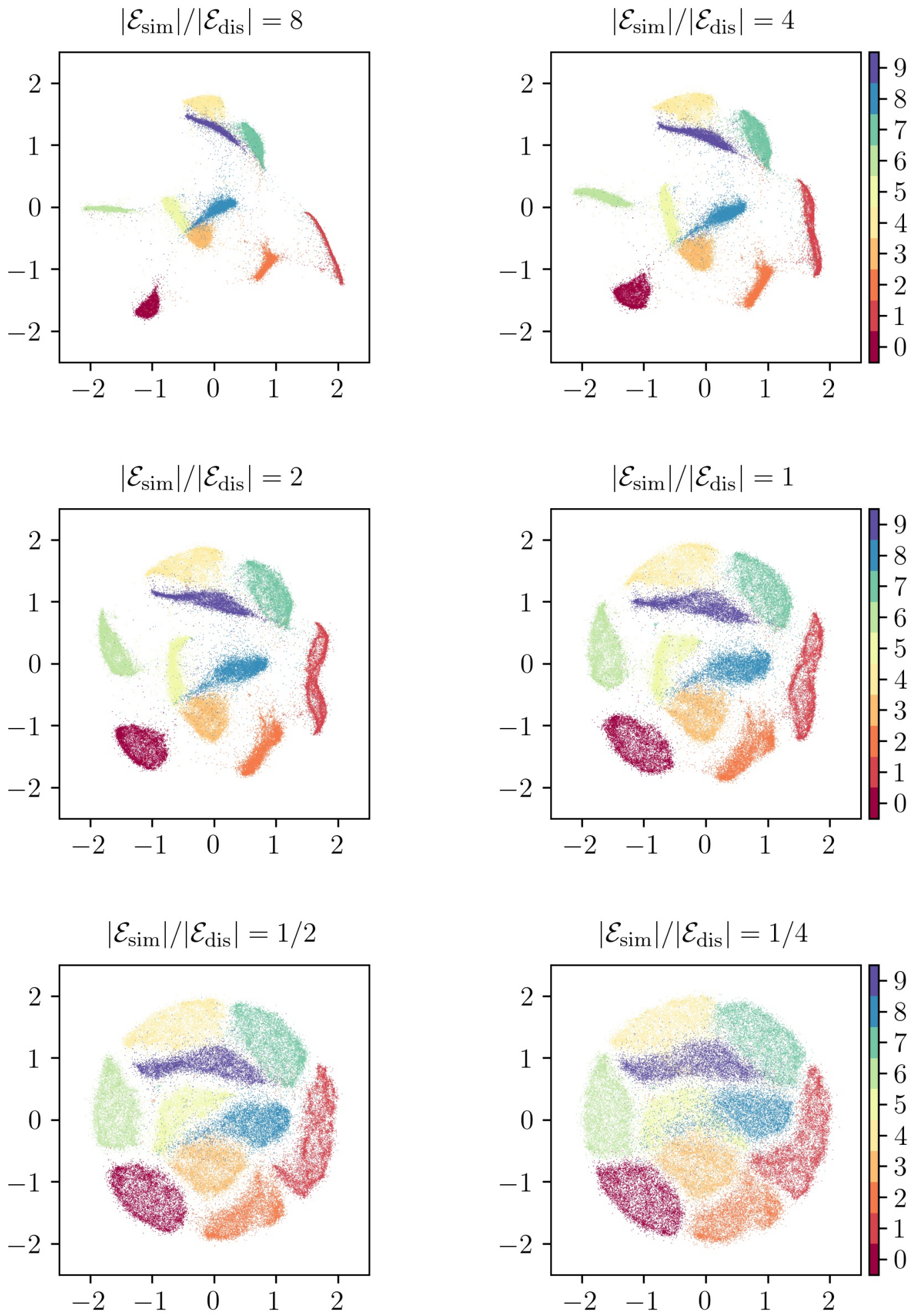

Figure 8.6: Varying $\left|\mathcal{E}_{\text {dis }}\right|$. Standardized embeddings of MNIST, varying $\left|\mathcal{E}_{\text {dis }}\right|$. 

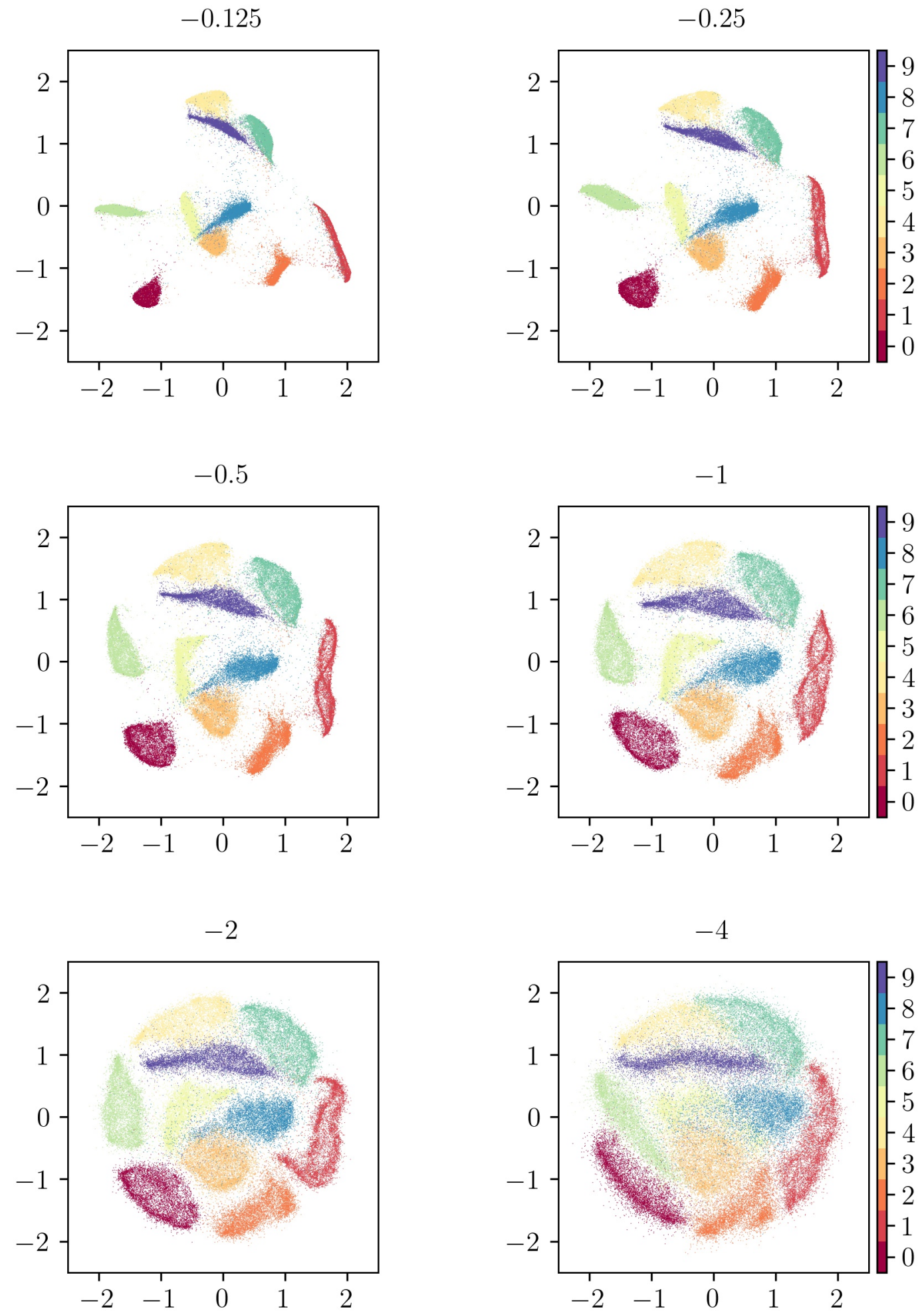

Figure 8.7: Varying negative weights. Standardized embeddings of MNIST, varying negative weights. 

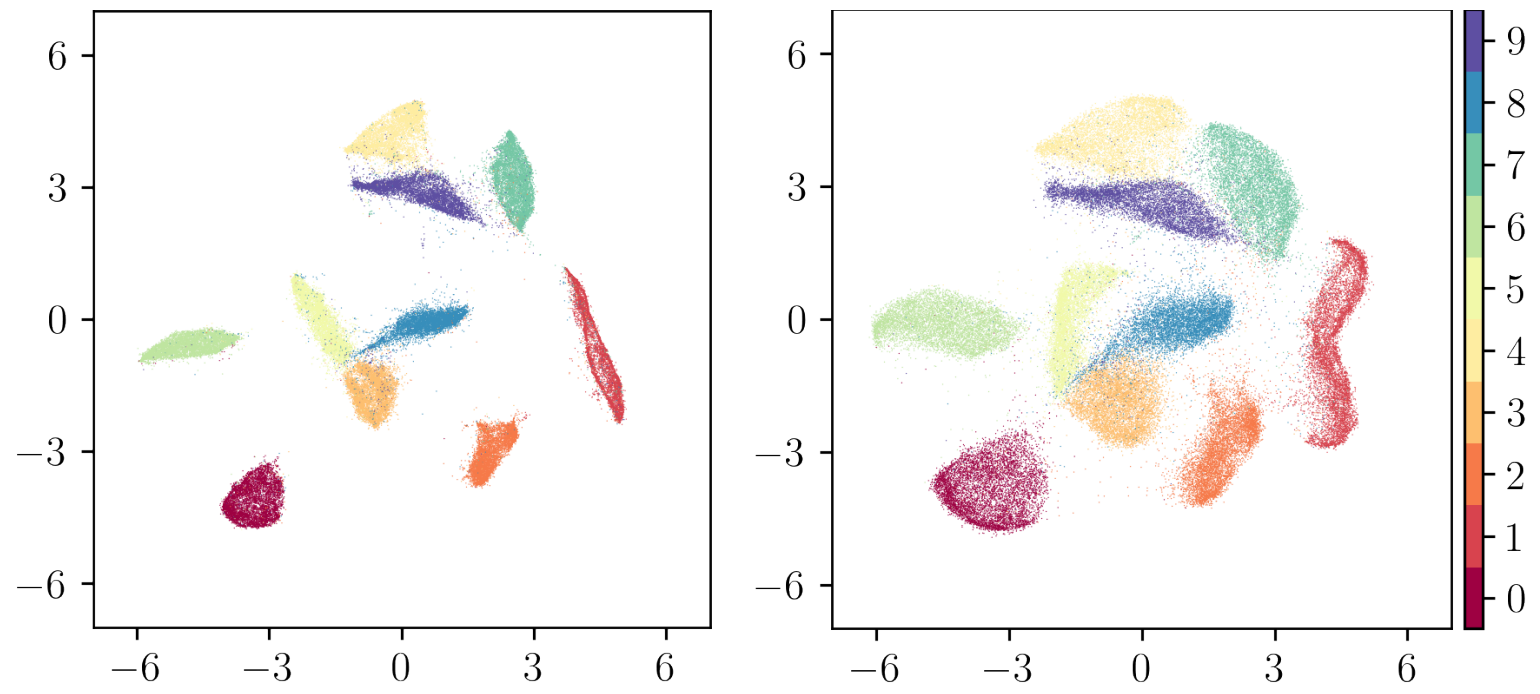

Figure 8.8: More embeddings. Centered embeddings of MNIST with log-one-plus (left) and Huber (right) attractive penalties, and logarithmic repulsive penalties.

Centered embeddings. We solve two centered MDE problems and compare their solutions. The first MDE problem has a log-one-plus attractive penalty (4.3) ( $\alpha=$ $1.5)$, and the second has a Huber attractive penalty $(4.2)(\tau=0.5)$. These problems were solved in about 4 seconds on a GPU and 32 seconds on a CPU. The first problem was solved in 170 iterations, and the second was solved in 144 iterations.

The embeddings, shown in figure 8.8, are very similar. This suggests that the preprocessing (here, the choice of $\mathcal{E}_{\text {sim }}$ and $\mathcal{E}_{\text {dis }}$ ) has a strong effect on the embedding. The embedding produced using the Huber penalty has more points in the negative space between classes of digits. This makes sense, since for large $d$, the Huber penalty more heavily discourages large embedding distances (for similar points) than does the log-one-plus penalty. Moreover, the neighborhood graph is connected, so some points must be similar to multiple classes of digits. 

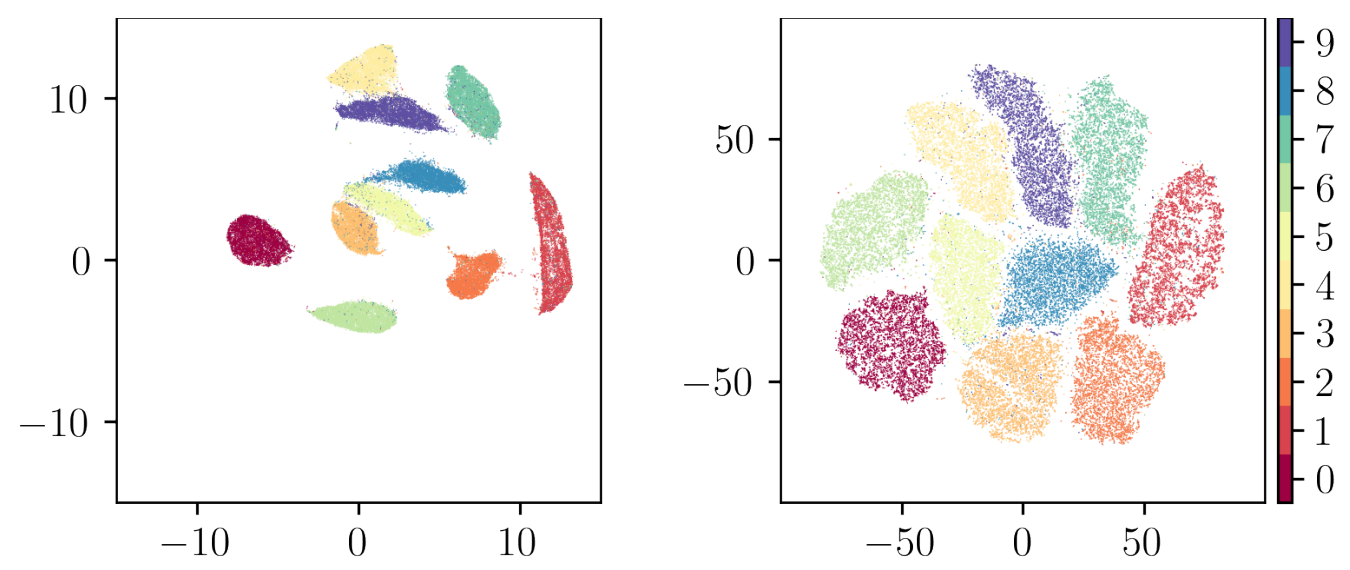

Figure 8.9: UMAP (left) and t-SNE (right) embeddings of MNIST.

\subsubsection{Comparison to other methods}

For comparison, we embed MNIST with UMAP [MHM18] and t-SNE [HR03], two popular embedding methods, using the umap-learn and openTSNE [PSZ19] implementations. Both methods start with neighborhood graphs on the original data, based on the Euclidean distance; umap-learn uses the nearest 15 neighbors of each image, and openTSNE uses the nearest 90 neighbors of each image. The UMAP optimization routine takes 20 seconds on our CPU, and the openTSNE optimization routine takes roughly one minute. (Neither implementation supports GPU acceleration.) On similar problems (the centered embeddings from §8.3.2), PyMDE takes roughly 30 seconds on CPU.

Figure 8.9 shows the embeddings. Note that the UMAP embedding bears a striking resemblance to the previously shown centered embeddings (figure 8.8). This is not surprising, since UMAP solves an unconstrained optimization problem derived from a similar graph and with similar distortion functions. 


\section{Chapter 9}

\section{Networks}

The MDE problems we formulate in this chapter are derived from networks. We start with an undirected (possibly weighted) network on $n$ nodes, in which the length of the shortest path between nodes $i$ and $j$ encodes an original distance between them. Such networks frequently arise in practice: examples include social networks, collaboration networks, road networks, web graphs, and internet networks. Our goal is to find an embedding of the nodes that preserves the network's global structure.

The specific network under consideration in this chapter is a co-authorship network: each node corresponds to a unique author, and two nodes are adjacent if the corresponding authors have co-authored at least one publication.

\subsection{Data}

We compiled a co-authorship network by crawling Google Scholar [Goo], an online bibliography cataloging research papers from many different fields. Each cataloged author has a profile page, and each page contains metadata describing the author's body of work. We crawled these pages to create our network. Our network contains 590,028 authors and 3,812,943 links. We additionally collected each author's unique identifier, affiliation, self-reported interests (such as machine learning, renewable energy, or high energy physics), h-index [Hir05], and cumulative number of citations. Our dataset is publicly available, at https://pymde.org.

We will compute two embeddings in this chapter: an embedding on the full network, and an embedding on a network containing only high-impact authors, which we define as authors with h-indices 50 or higher. The subgraph induced by highimpact authors has 44,682 vertices and 210,681 edges.

When constructing the embeddings, we use only the graph distances between 
nodes. Later in this section we describe how we categorize authors into academic disciplines using their interests. An author's academic discipline is the held-out attribute we use to check if the embedding makes sense.

Crawling. The data was collected in November 2020 by exploring Google Scholar with a breadth-first search, terminating the search after one week. The links were obtained by crawling authors' listed collaborators. Google Scholar requires authors to manually add their coauthors (though the platform automatically recommends co-authors); as a result, if two authors collaborated on a paper, but neither listed the other as a co-author, they will not be adjacent in our network. As a point of comparison, a co-authorship network of Google Scholar from 2015 that determined co-authorship by analyzing publication lists contains 409,392 authors and 1,234,019 links $\left[\mathrm{Che}^{+} 17\right]$.

While our co-authorship network faithfully represents Google Scholar, it deviates from reality in some instances. For example we have found that a small number of young researchers have listed long-deceased researchers such as Albert Einstein, John von Neumann, Isaac Newton, and Charles Darwin as co-authors. Compared to the size of the network, the number of such anomalies is small; e.g., there are only thirty authors who are adjacent to Einstein but not among his true co-authors. As such we have made no attempt to remove erroneous links or duplicated pages (Einstein has two). For a more careful study of academic collaboration, see [Che $\left.{ }^{+} 17\right]$.

Some basic properties. The diameter of the full network (the length of the longest shortest path) is 10 . The diameter of the network induced by high-impact authors is 13 .

Figure 9.1 shows the CDFs of h-indices and citation counts. The median h-index is 14 , the 95 th percentile is 58 , and the maximum is 303 ; the median citation count is 891 , the 95th percentile is 1616 , and the maximum is $1,107,085$. The maximum h-index and maximum citation count both belong to the late philosopher Michel Foucault.

Figure 9.2 shows the CDF of node degrees for the full network and the subgraph induced by high-impact authors. The number of collaborators an author has is moderately correlated with h-index (the Pearson correlation coefficient is 0.44 ); citation count is strongly correlated with h-index (0.77).

The data includes 280,819 unique interests, with 337,514 authors interested in at least one of the 500 most popular interests. The top five interests are machine learning (listed by 38,220 authors), computer vision $(15,123)$, artificial intelligence $(14,379)$, deep learning $(8,984)$, and bioinformatics $(8,496)$. 

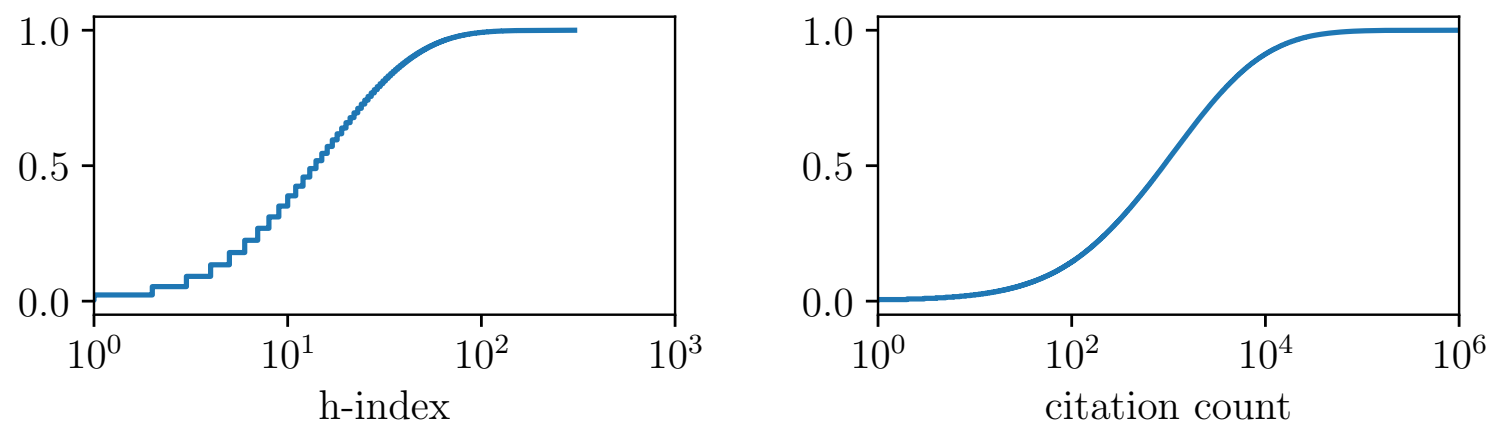

Figure 9.1: CDFs of author h-indices and citation counts

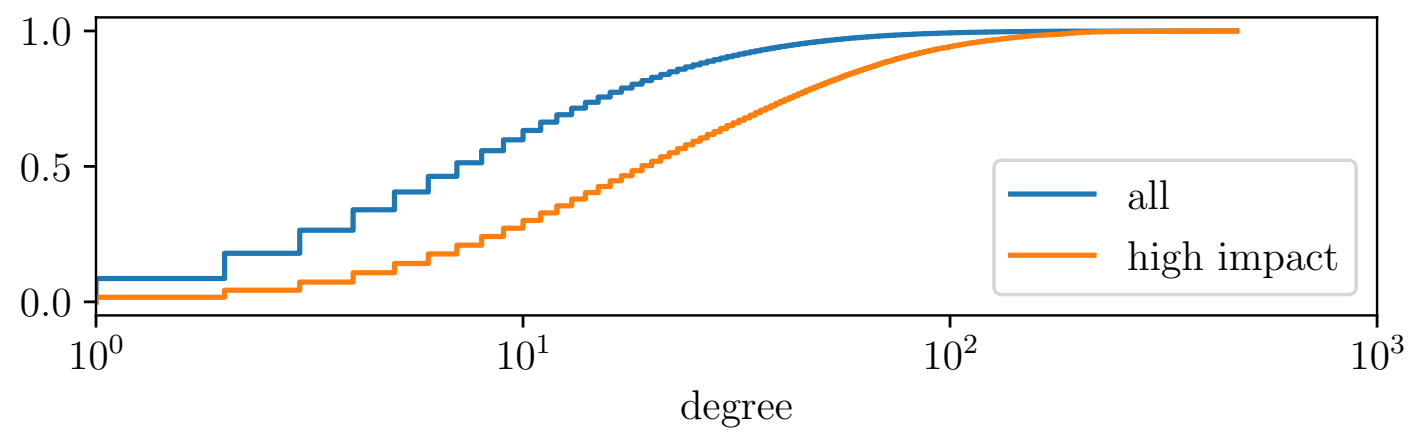

Figure 9.2: CDFs of node degree (co-author count) for the full network and the high-impact author network. 
Academic disciplines. We assigned the high-impact authors to one of five academic disciplines, namely biology, physics, electrical engineering, computer science, and artificial intelligence, in the following ad-hoc way. First, we manually labeled the 500 most popular interests with the academic discipline to which they belonged (interests that did not belong to any of the five disciplines were left unlabeled). Then, we scanned each author's interests in the order they chose to display them on their profile, assigning the author to the discipline associated with their first labeled interest. For example, authors interested in machine learning first and bioinformatics second were assigned to artificial intelligence, while authors interested in bioinformatics first and machine learning second were assigned to biology. Authors that did not have at least one labeled interest were ignored. This left us with 16,569 high-impact authors labeled with associated academic disciplines, out of 44,682. 

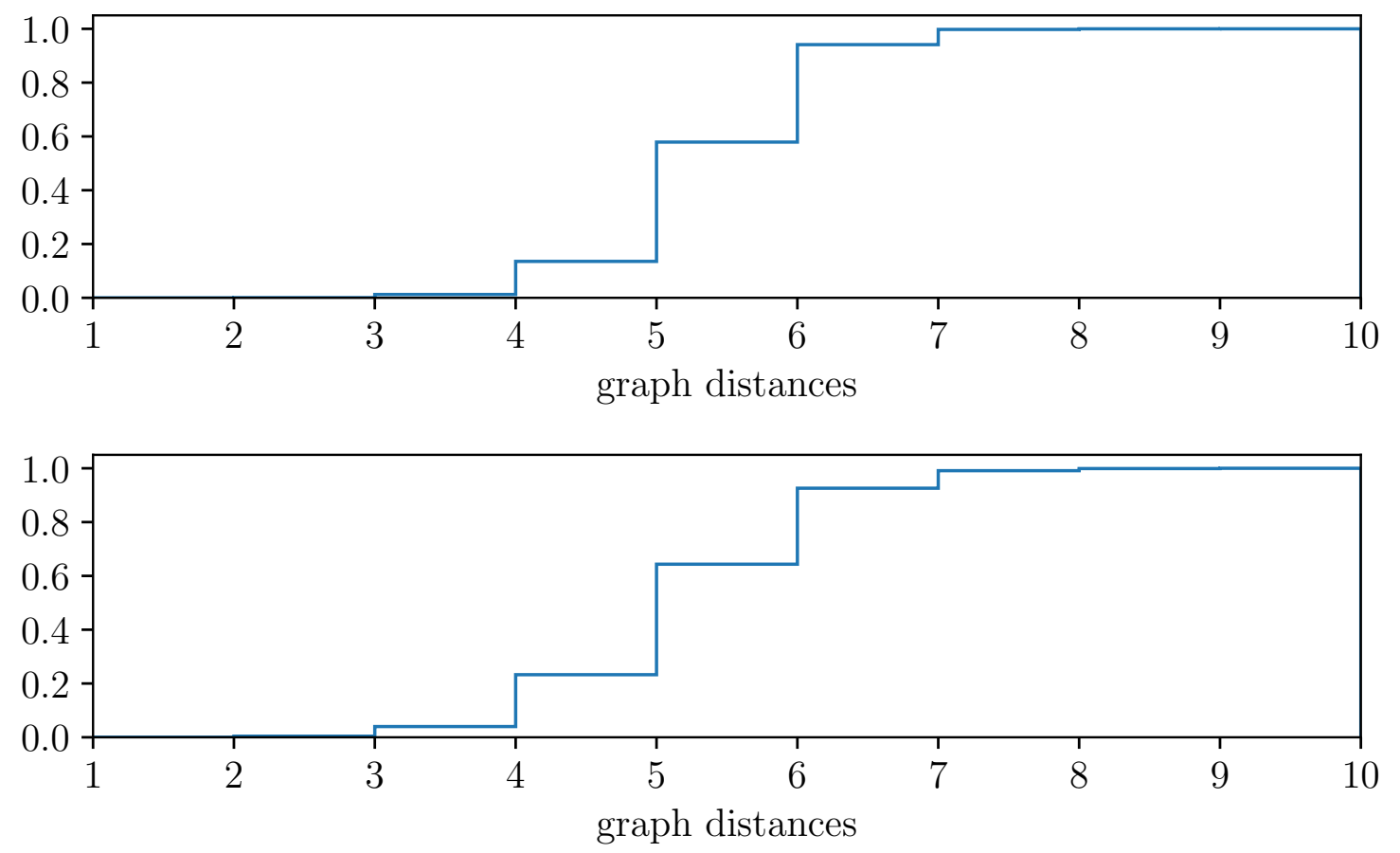

Figure 9.3: CDFs of graph distances. Top. All authors. Bottom. High-impact authors.

\subsection{Preprocessing}

We compute graph distances between all pairs of authors. On the full network, this yields approximately 174 billion distances, or roughly 696 GB of data, too much to store in the memory of our machine. To make the embedding tractable, we sample a small fraction of the graph distances uniformly at random, choosing to retain 0.05 percent of the distances. This results in a graph $\mathcal{E}_{\text {all }}$ on 590,028 vertices, with 87,033,113 edges. Computing all 174 billion distances and randomly subsampling them took seven hours using six CPU cores. We repeat this preprocessing on the high-impact network, retaining 10 percent of the graph distances. This yields a graph $\mathcal{E}_{\text {impact }}$ with 88,422,873 edges. Computing the graph distances with PyMDE took less than one minute, using six CPU cores.

The CDFs of graph distances are shown in figure 9.3. The values 4, 5, and 6 account for around $90 \%$ of the distances in both networks. (We will see artifacts of these highly quantized values in some of our embeddings.) 


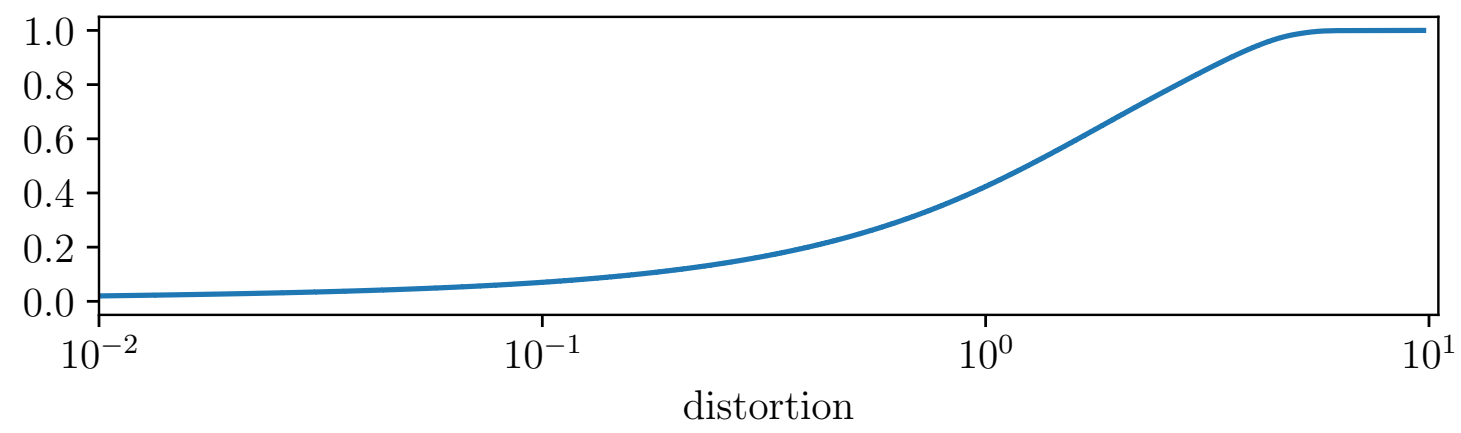

Figure 9.4: CDF of distortions for the embedding on all authors.

\subsection{Embedding}

We solve two unconstrained MDE problems, based on $\mathcal{E}_{\text {all }}$ and $\mathcal{E}_{\text {impact }}$. The MDE problems are derived from original deviations, with $\delta_{i j}$ being the graph distance between $i$ and $j$. For both, we use the absolute loss (4.5) and embed into $\mathbf{R}^{2}$.

\subsubsection{All authors}

The embedding on $\mathcal{E}_{\text {all }}$ has distortion 1.58. The embedding was computed in 300 iterations by PyMDE, to a residual norm of $1.9 \times 10^{-5}$. This took 71 seconds on our GPU.

Figure 9.4 shows a CDF of the the distortions. The five largest distortions all belong to pairs whose true distance is 9 , but whose embedding distances are small (the pair with the largest distortion includes a philosopher and an automatic control engineer). The authors in these pairs are "unimportant" in that they have very few co-authors, and lie on the periphery of the embedding.

Figure 9.5 shows the embedding. (Plots of embeddings in this chapter have black backgrounds, to make various features of the embeddings easier to see.) Each embedding vector is colored by its author's degree in the co-authorship network; the brighter the color, the more co-authors the author has. The embedding appears sensible: highly collaborative authors are near the center, with the degree decreasing as one moves outward. The diameter of the embedding is approximately 13 (measured in Euclidean norm), compared to the original network's 10 (measured in graph distance). 


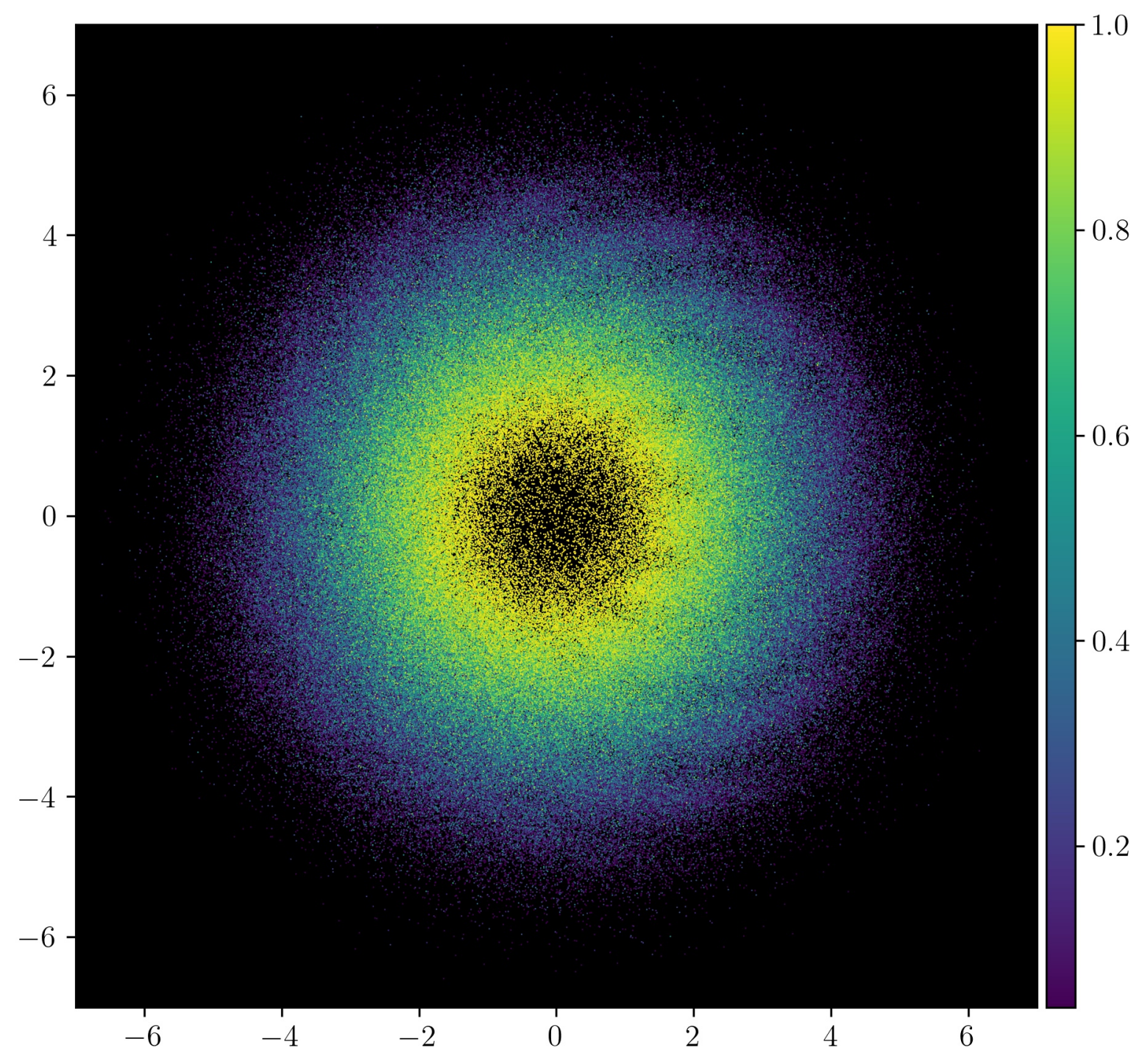

Figure 9.5: Co-authorship network. An embedding of a co-authorship network from Google Scholar, colored by degree percentile. The brighter the color, the more coauthors the author has. The network has 590,028 authors. 


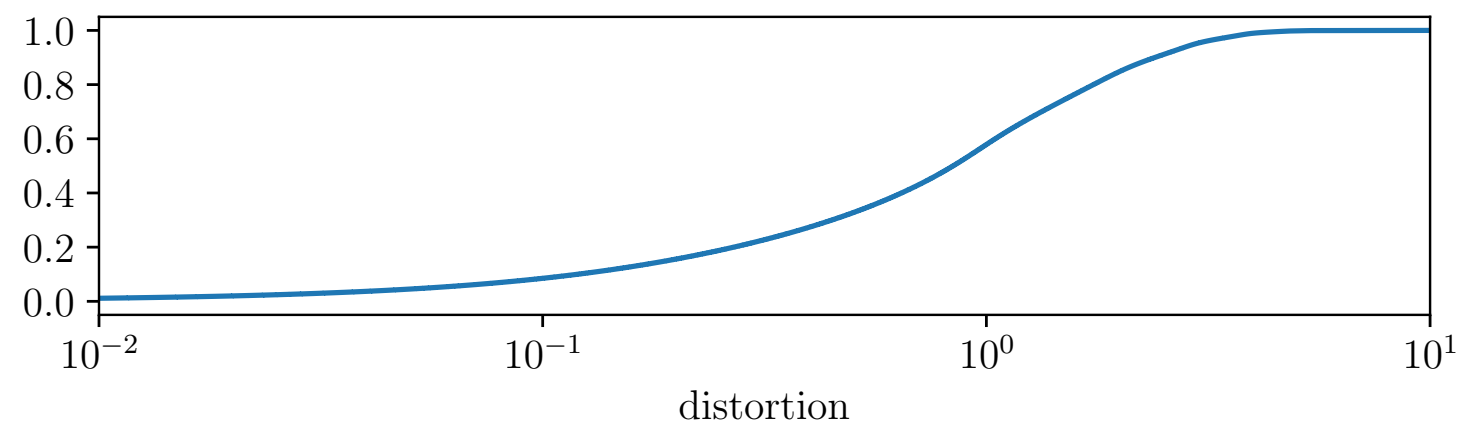

Figure 9.6: CDF of distortions for the embedding on high-impact authors.

\subsubsection{High-impact authors}

An embedding on the graph $\mathcal{E}_{\text {impact }}$ has average distortion 1.45 (figure 9.6 shows a $\mathrm{CDF}$ of the distortions). PyMDE computed this embedding on a GPU in 54 seconds, in 173 iterations.

Figure 9.7 shows the embedding, colored by degree. Highly collaborative authors are near the center, with collaboration decreasing radially from the center. The diameter is approximately 16, compared to the original network's 13.

The embedding has an interesting structure, with many intersecting arcs. These arcs are likely due to the original distances taking on highly quantized values (see figure 9.3). In figure 9.8 we overlay a large fraction of the original high-impact network's edges onto the embedding (i.e., edges $(i, j)$ for which $\delta_{i j}=1$ ); we show 50,000 edges, sampled uniformly at random from the original 210,681. The edges trace out the arcs, and connect adjacent communities of highly collaborative authors.

Academic disciplines. Figure 9.9 shows the embedding vectors colored by academic discipline (authors that are not tagged with a discipline are omitted). Purple represents biology, orange represents physics, green represents electrical engineering, cyan represents computer science, and red represents artificial intelligence.

Authors are grouped by academic discipline, and similar disciplines are near each other. Additionally, it turns out nearby curves in an academic discipline roughly correspond to sub-disciplines. For example, authors in the purple curves toward the center of the embedding are largely interested in ecology, while authors in the outer purple curves are more interested in genomics and medicine.

Most highly-collaborative authors (authors near the center of the embedding) study computer science, artificial intelligence, or biology. This is consistent with a 
previous study of Google Scholar [Che $\left.{ }^{+} 17\right]$, which found that computer scientists and biologists are disproportionately important in the co-authorship network, as measured by metrics such as degree and PageRank $\left[\mathrm{Pag}^{+} 99\right]$. 


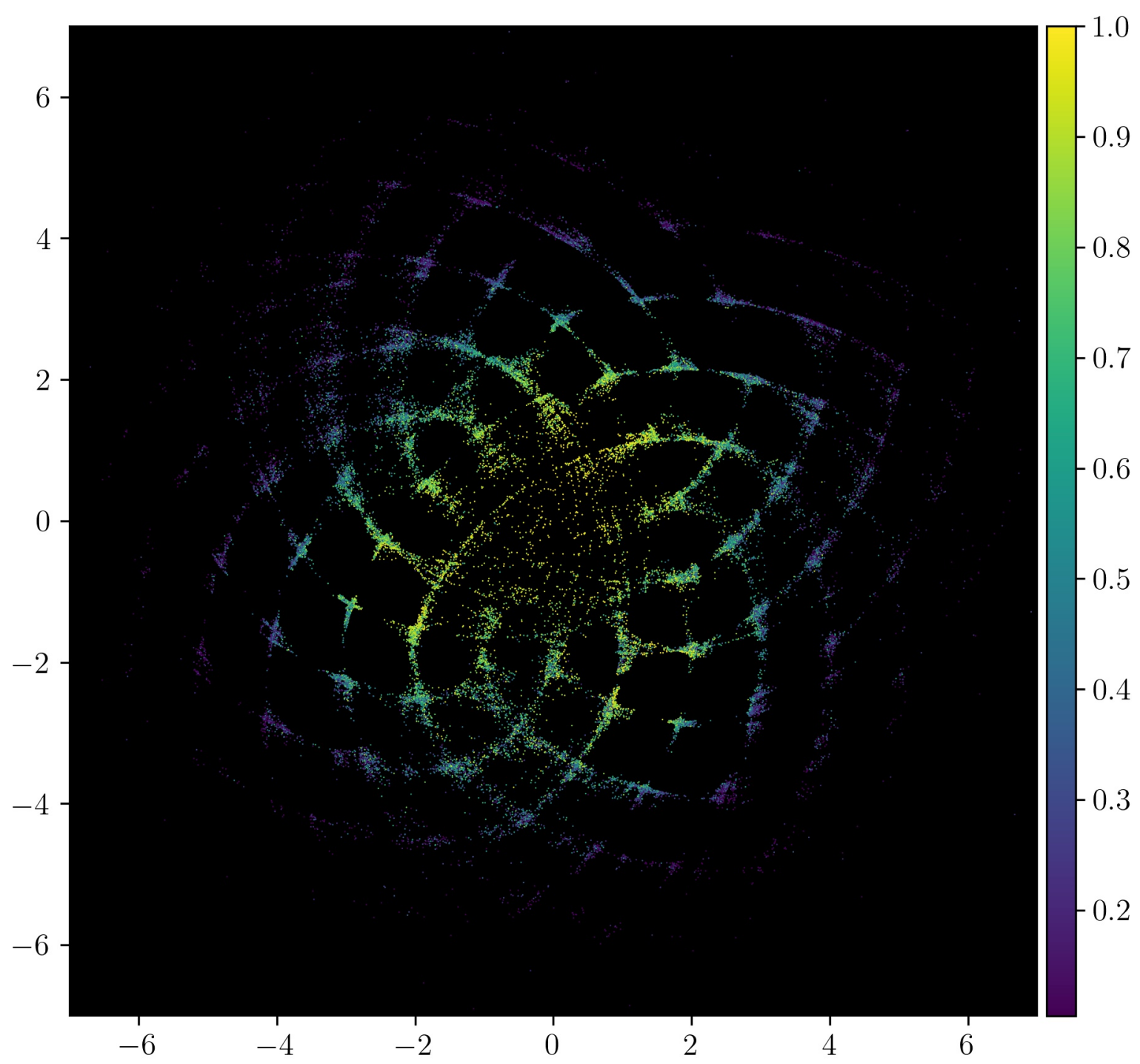

Figure 9.7: High-impact authors. An embedding of a co-authorship network on authors with an h-index of 50 or higher, colored by degree percentile. 


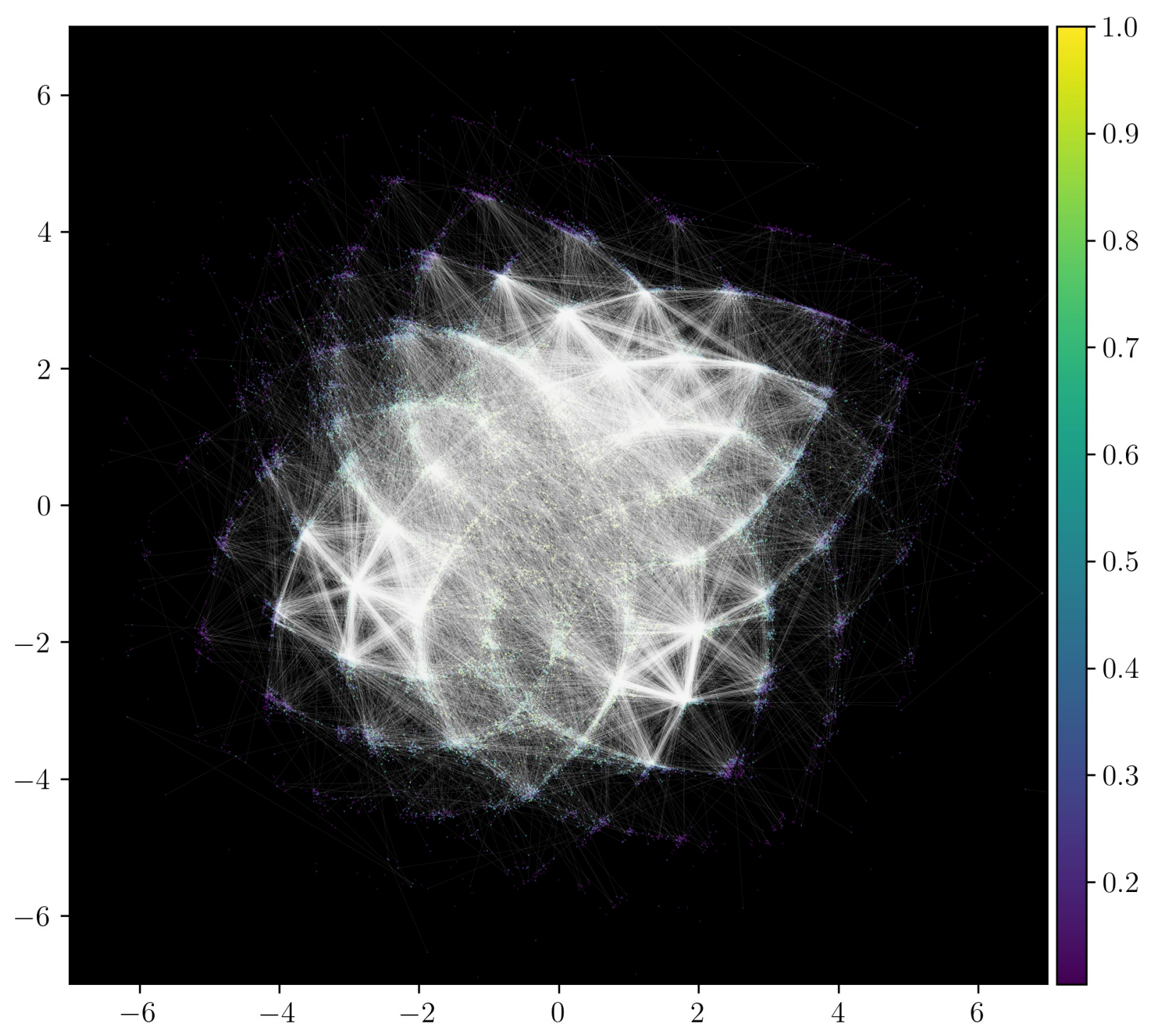

Figure 9.8: High-impact authors, with links. High-impact author embedding, colored by degree percentile, with links between co-authors displayed as white line segments. 


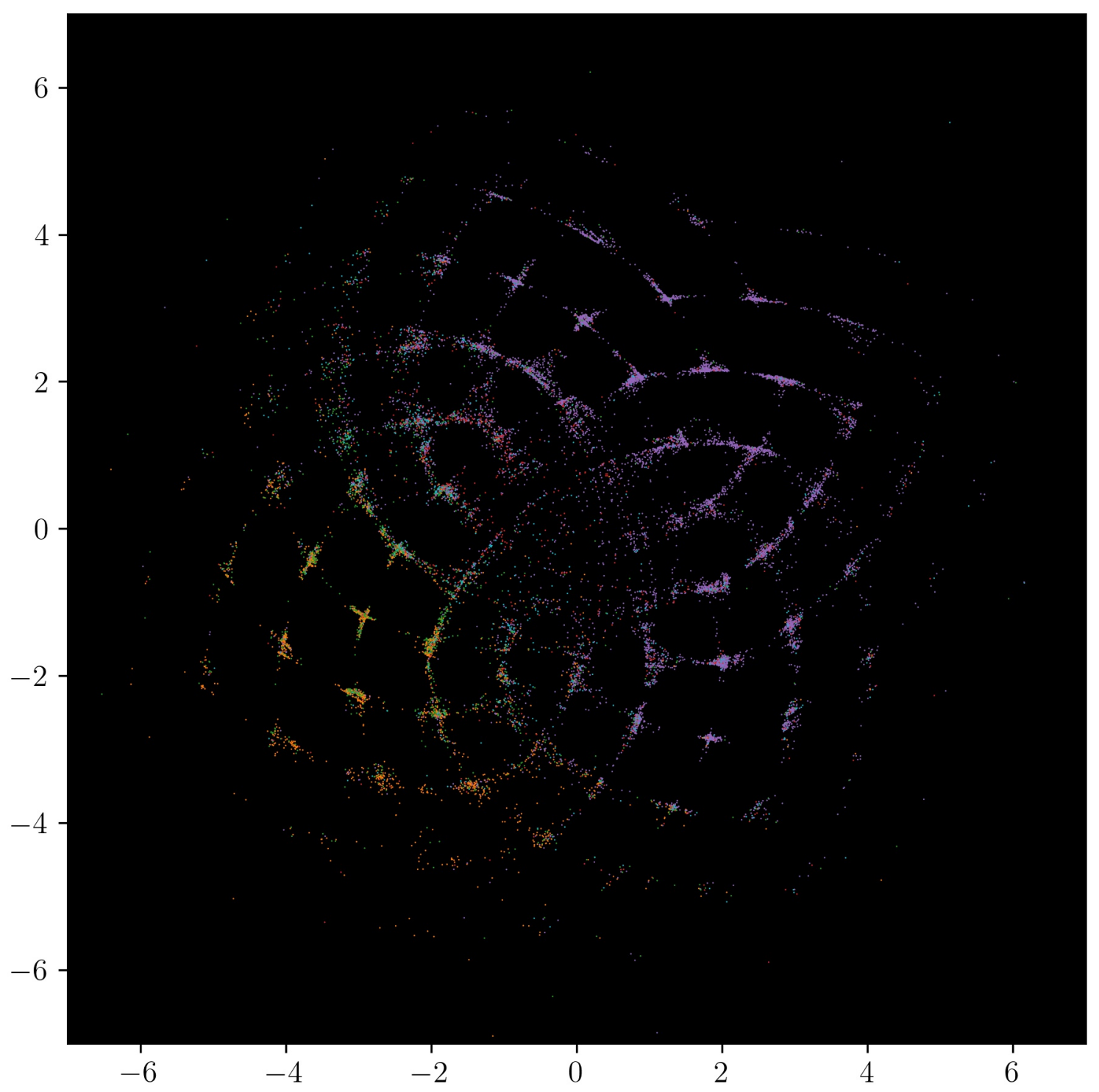

Figure 9.9: Academic disciplines. High-impact author embedding, colored by academic discipline. The disciplines are biology (purple), physics (orange), electrical engineering (green), computer science (cyan), and artificial intelligence (red). 


\subsubsection{Comparison to other methods}

For comparison, we embed the high-impact co-authorship networks using UMAP [MHM18] and t-SNE [MH08], using the umap-learn and the scikit-learn [ $\left.\mathrm{Ped}^{+} 11\right]$ implementations. We give UMAP and t-SNE the distance matrices containing the graph distances associated with $\mathcal{E}_{\text {impact }}$, and we use the default hyper-parameters set by the software.

The embeddings are shown below in figure 9.10, colored by degree and academic discipline. Neither method has preserved the global structure of the network, but both have organized the embedding vectors by discipline. (As we have seen, embeddings emphasizing local structure over global structure are readily produced with PyMDE; in this chapter, however, we chose to emphasize global structure instead.)
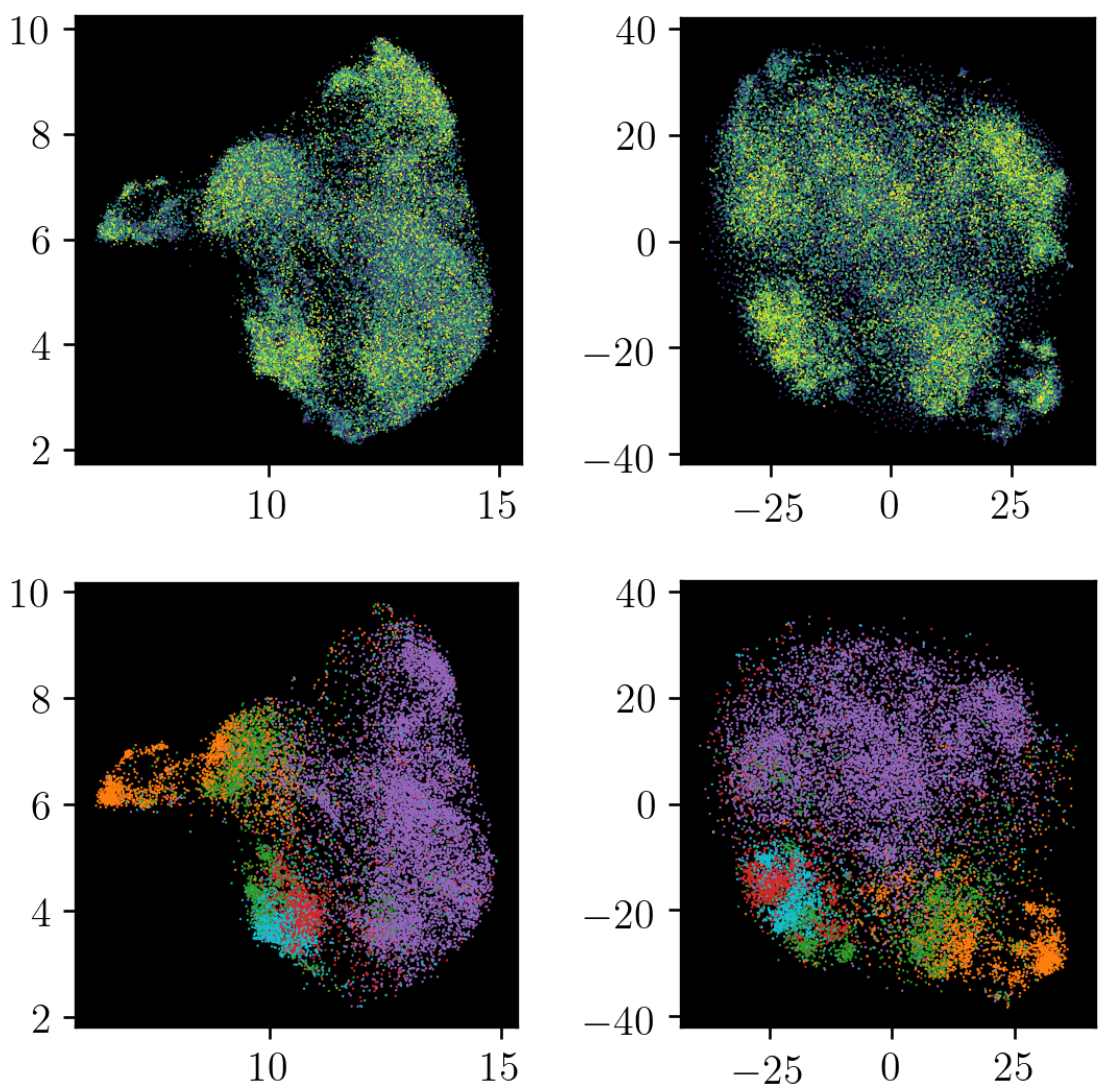

Figure 9.10: UMAP (left) and t-SNE (right) embeddings of the high-impact author network, colored by degree (top) and academic discipline (bottom). 


\section{Chapter 10}

\section{Counties}

In this example, we embed demographic data for 3,220 United States counties into $\mathbf{R}^{2}$. The data broadly describes the composition of the counties, and contains information about gender, ethnicity, and income (among other things). To make sure our embeddings make sense, we will use an additional attribute for each county to validate the embeddings, which is the fraction who voted Democratic in the 2016 election. (We do not use this attribute in forming the embeddings.)

\subsection{Data}

Our raw data comes from the 2013-2017 American Community Survey (ACS) 5-Year Estimates [Uni], which is a longitudinal survey run by the United States Census Bureau that records demographic information about the $n=3,220$ US counties. We will use 34 of the demographic features to create our embedding of US counties.

\subsection{Preprocessing}

We carry out some standard preprocessing of the raw features, listed in table 10.1. For positive features that range over a very wide scale, such as household income, we apply a log-transform, i.e., replace the raw feature with its logarithm. We normalize raw features that are counts, i.e., divide them by the county population. (We do not transform features that are given as fractions or proportions.) After these transformations, we standardize each feature to have zero mean and standard deviation one over the counties.

Using these preprocessed features, we generate a $k$-nearest (Euclidean) neighbor 


\begin{tabular}{|c|c|}
\hline Feature & Transformation \\
\hline Number of people & Log-transform \\
\hline Number of voting age citizens & Log-transform \\
\hline Number of men & Normalize \\
\hline Number of women & Normalize \\
\hline Fraction Hispanic & None \\
\hline Fraction White & None \\
\hline Fraction Black & None \\
\hline Fraction Native American or Alaskan & None \\
\hline Fraction Asian & None \\
\hline Fraction Native Hawaiian or Pacific Islander & None \\
\hline Median household income & Log-transform \\
\hline Median household income standard deviation & Log-transform \\
\hline Household income per capita & Log-transform \\
\hline Household income per capita standard deviation & Log-transform \\
\hline Fraction below the poverty threshold & None \\
\hline Fraction of children below the poverty threshold & None \\
\hline $\begin{array}{l}\text { Fraction working in management, business, science, or } \\
\text { the arts }\end{array}$ & None \\
\hline Fraction working in a service job & None \\
\hline Fraction working in an office or sales job & None \\
\hline $\begin{array}{l}\text { Fraction working in natural resources, construction, } \\
\text { and maintenance }\end{array}$ & None \\
\hline $\begin{array}{l}\text { Fraction working in production, transportation, and } \\
\text { material movement }\end{array}$ & None \\
\hline Fraction working in the private sector & None \\
\hline Fraction working in the public sector & None \\
\hline Fraction working for themselves & None \\
\hline Fraction doing unpaid family work & None \\
\hline Number that have a job (are employed) & Normalize \\
\hline Fraction unemployed & None \\
\hline $\begin{array}{l}\text { Fraction commuting to work alone in a car, van, or } \\
\text { truck }\end{array}$ & None \\
\hline Fraction carpooling in a car, van, or truck & None \\
\hline Fraction commuting via public transportation & None \\
\hline Fraction walking to work & None \\
\hline Fraction commuting via other means & None \\
\hline Fraction working at home & None \\
\hline Average commute time in hours & None \\
\hline
\end{tabular}

Table 10.1: List of the features in the ACS data set, along with the initial preprocessing transformation we applied. 
graph, with $k=15$, which gives a graph $\mathcal{E}_{\text {sim }}$ with 75,250 edges. We then sample, at random, another 75,250 pairs of counties not in $\mathcal{E}_{\text {sim }}$, to obtain a graph of dissimilar counties, $\mathcal{E}_{\text {dis }}$. Our final graph $\mathcal{E}=\mathcal{E}_{\text {sim }} \cup \mathcal{E}_{\text {dis }}$ has 150,500 edges, corresponding to an average degree of around 47 . We assign weight $w_{i j}=2$ for $(i, j) \in \mathcal{E}_{\text {sim }}$ when counties $i$ and $j$ are both neighbors of each other. We assign weight $w_{i j}=1$ for $(i, j) \in \mathcal{E}_{\text {sim }}$ when $i$ is a neighbor of $j$ but $j$ is not a neighbor of $i$ (or vice versa). We assign weight $w_{i j}=-1$ for $(i, j) \in \mathcal{E}_{\text {dis }}$.

\subsection{Embedding}

\subsubsection{PCA embedding}

We consider PCA first. The PCA embedding for $m=2$ is shown in figure 10.1, with each county shown as a colored dot, with colors depending on the fraction of voters who voted Democratic in 2016, from red (0\%) to blue (100\%). The embedding clearly captures some of the structure of the voting patterns, but we can see many instances where counties with very different voting appear near each other in the embedding. (The blue counties are urban, and far more populous than the many rural red counties seen in the embedding. The total number of people who voted Democratic exceeded those who voted Republican.)

\subsubsection{Unconstrained embedding}

Next we solve an unconstrained (centered) MDE problem based on the graph $\mathcal{E}=$ $\mathcal{E}_{\text {sim }} \cup \mathcal{E}_{\text {dis }}$. We use the log-one-plus penalty (4.3) $(\alpha=2)$ for $p_{\mathrm{s}}$ and the logarithmic penalty (4.4) for $p_{\mathrm{d}}(\alpha=0.5)$. We choose embedding dimension $m=2$.

Figure 10.2 shows the results. Compared to the PCA embedding, we see a better separation of regions corresponding to heavily Democratic, heavily Republican, and mixed voting. Figures 10.3 and 10.4 take a closer look at the upper right and bottom of the plot, labeling some representative counties. We can also see a few swing counties, i.e., counties that historically vote Democratic, but recently lean Republican (or vice versa), near the strongly Democratic (or Republican) counties; these counties are colored light red (or blue), because they have weak majorities.

We give two alternative visualizations of the embedding in figure 10.5. In these visualizations, we color each county according to its value in each of the two embedding coordinates. In figure 10.6, we show the counties colored with the fraction of people who actually voted Democratic in the 2016 election, as a point of reference. 


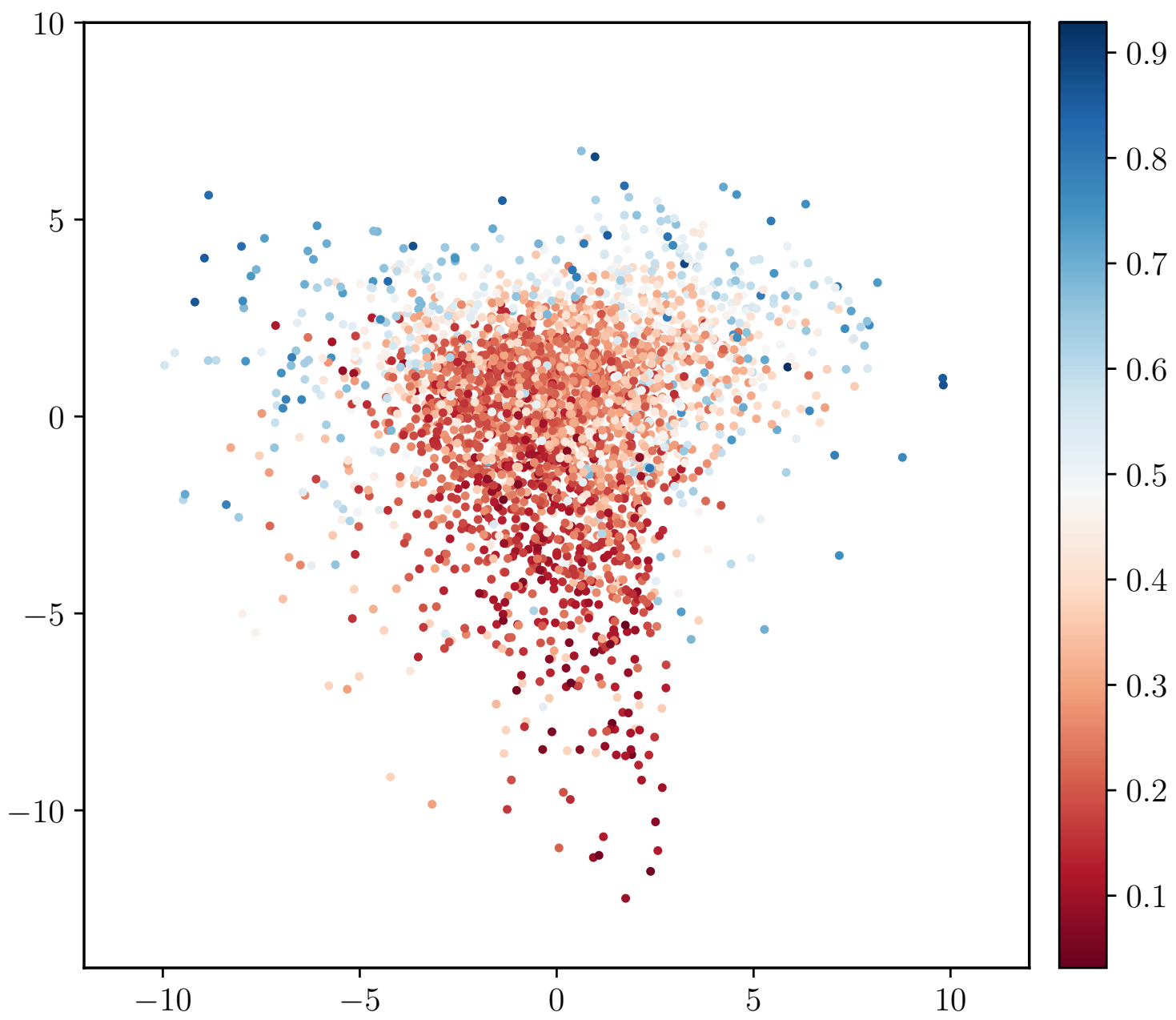

Figure 10.1: PCA embedding of the election data, colored by the fraction of voters in each county who voted Democratic in the 2016 United States presidential election, from red $(0 \%)$ to blue $(100 \%)$. 


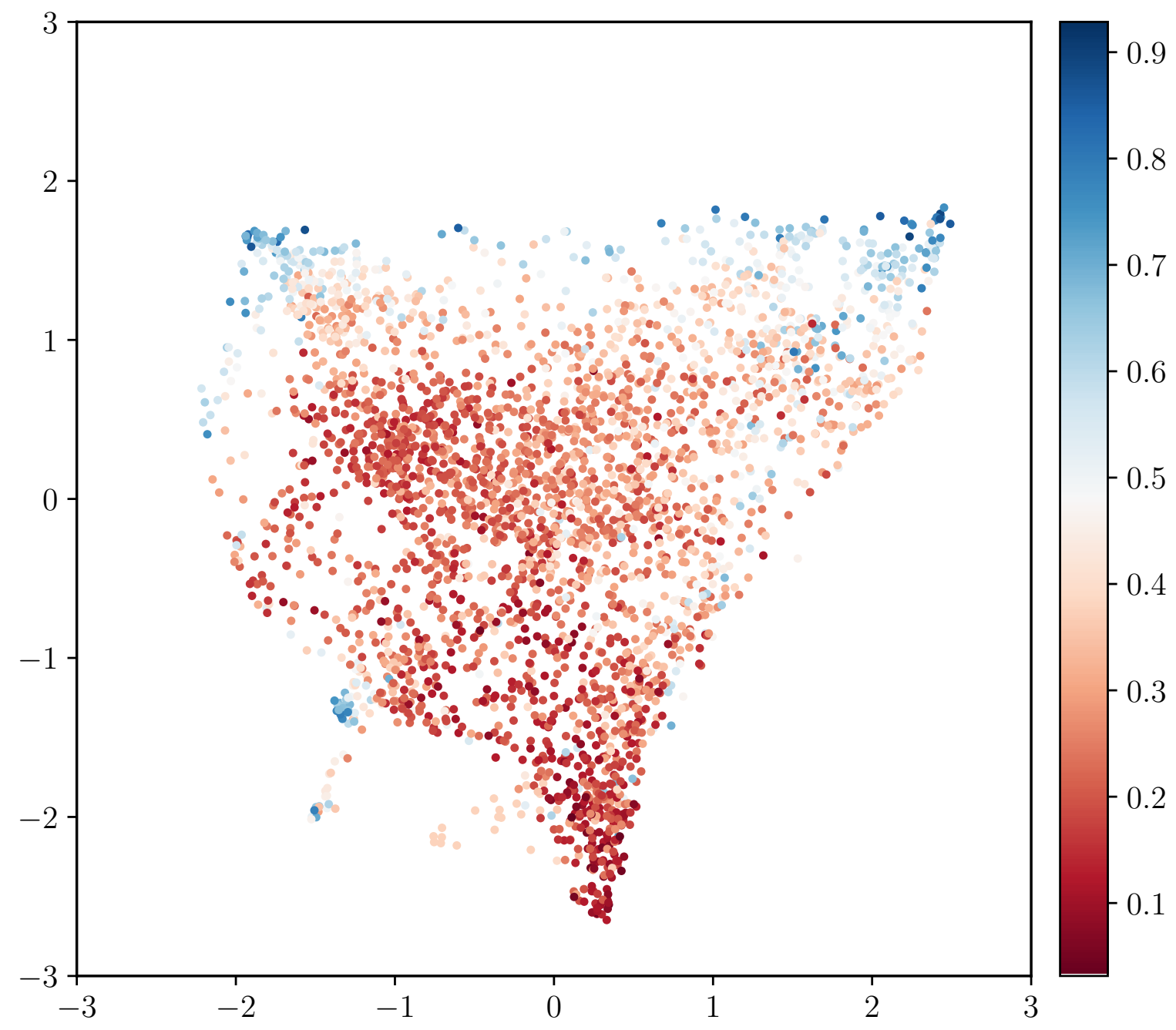

Figure 10.2: Embedding. A centered embedding of the election data, with log-oneplus attractive and logarithmic repulsive penalty functions. 


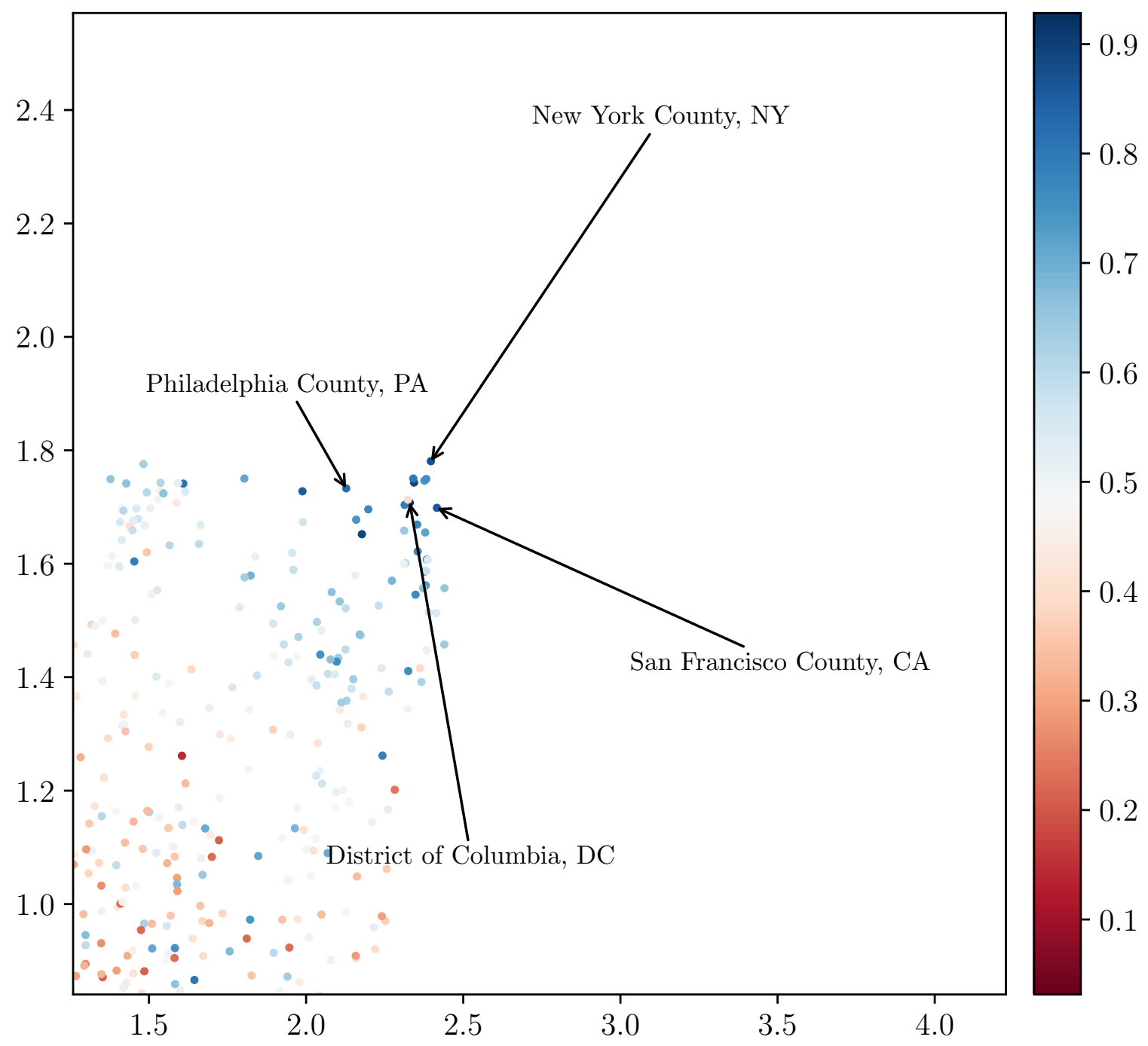

Figure 10.3: Upper right corner. A closer look at the upper right corner of the embedding shown in figure 10.2. Several densely populated urban counties appear. 


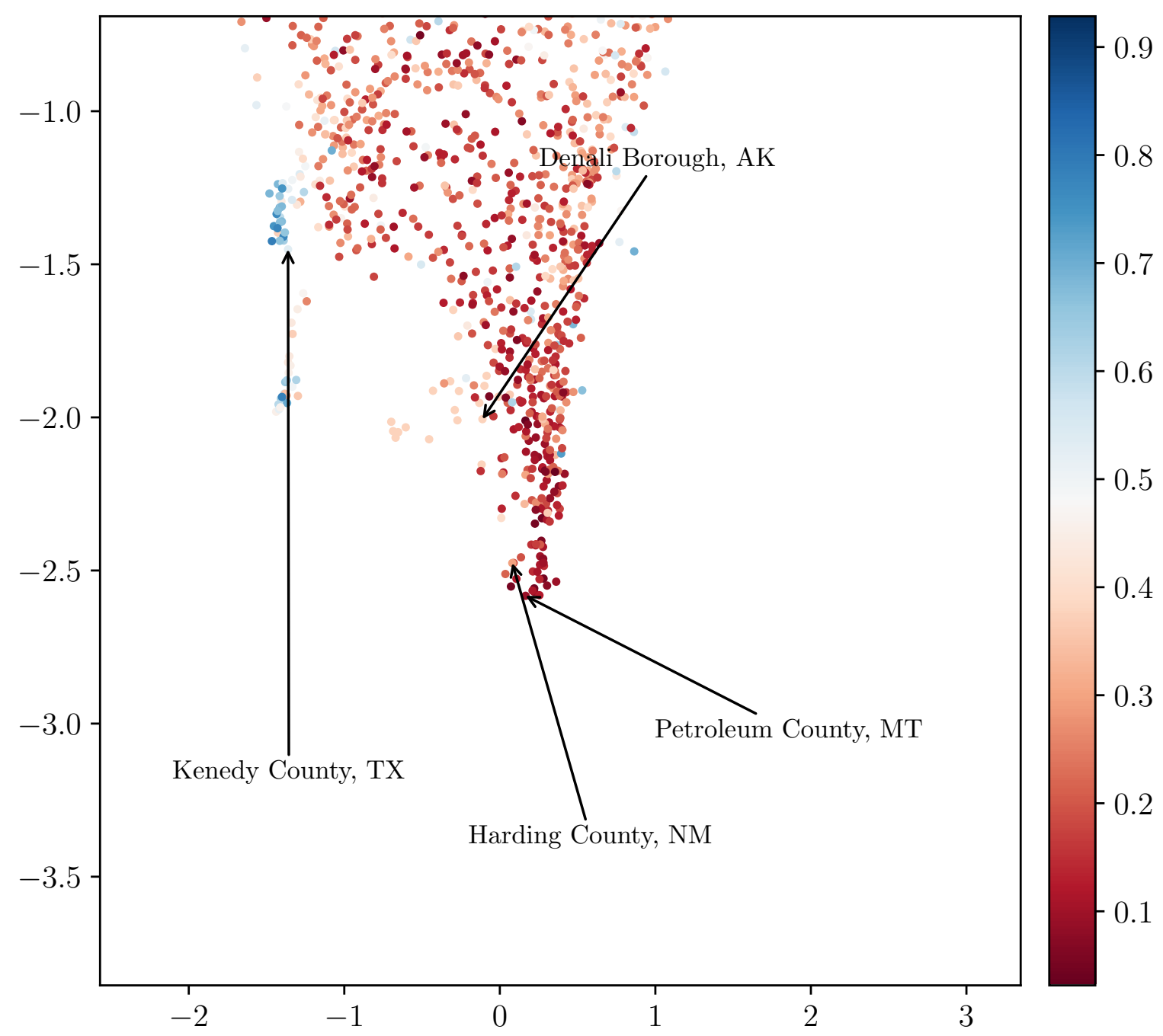

Figure 10.4: Bottom. A closer look at the bottom of the embedding shown in figure 10.2. Several sparsely populated rural counties appear. 

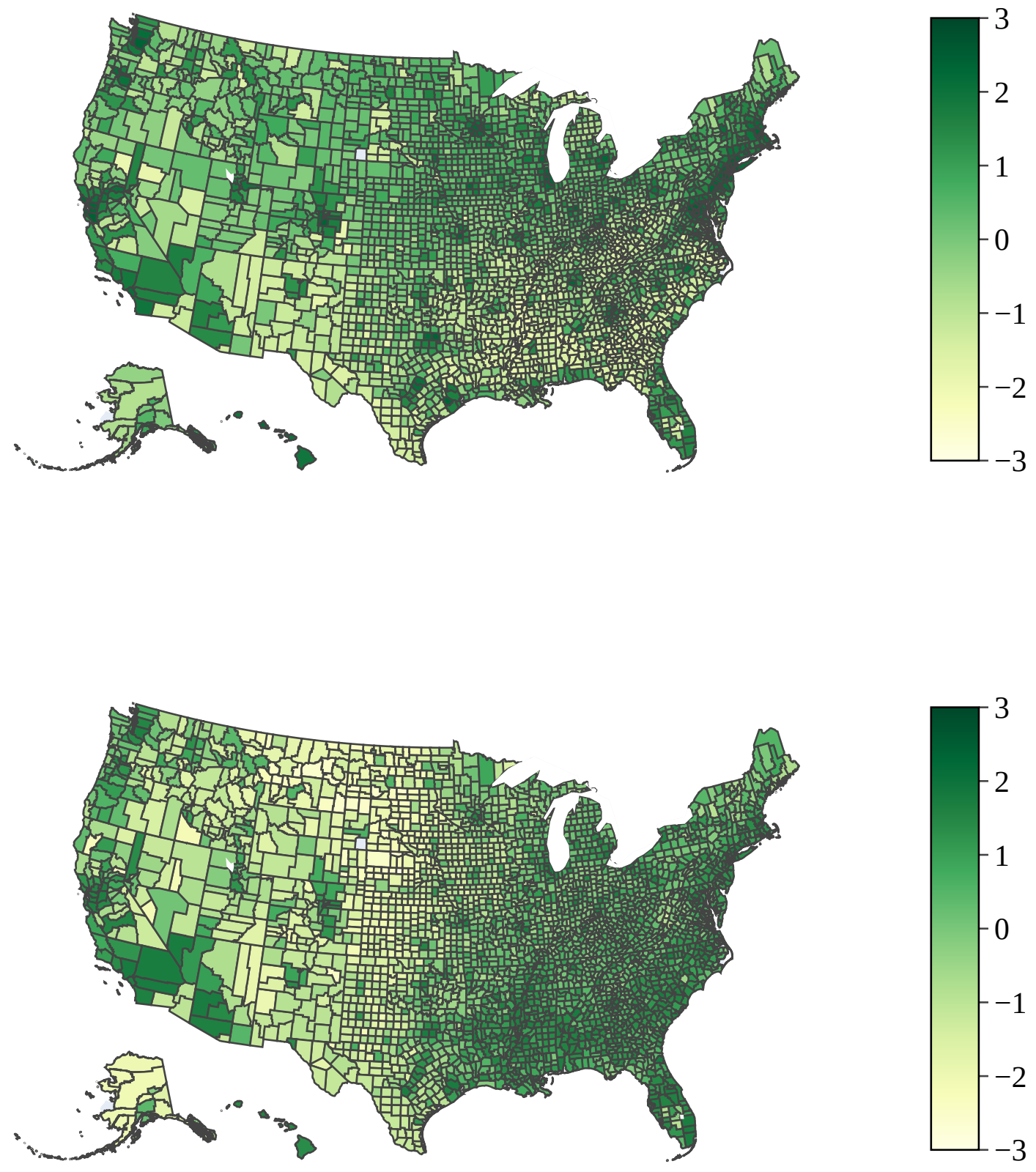

Figure 10.5: Embedding by coordinate. Counties colored by their first (top) and second (bottom) coordinate of the embedding from figure 10.2. 


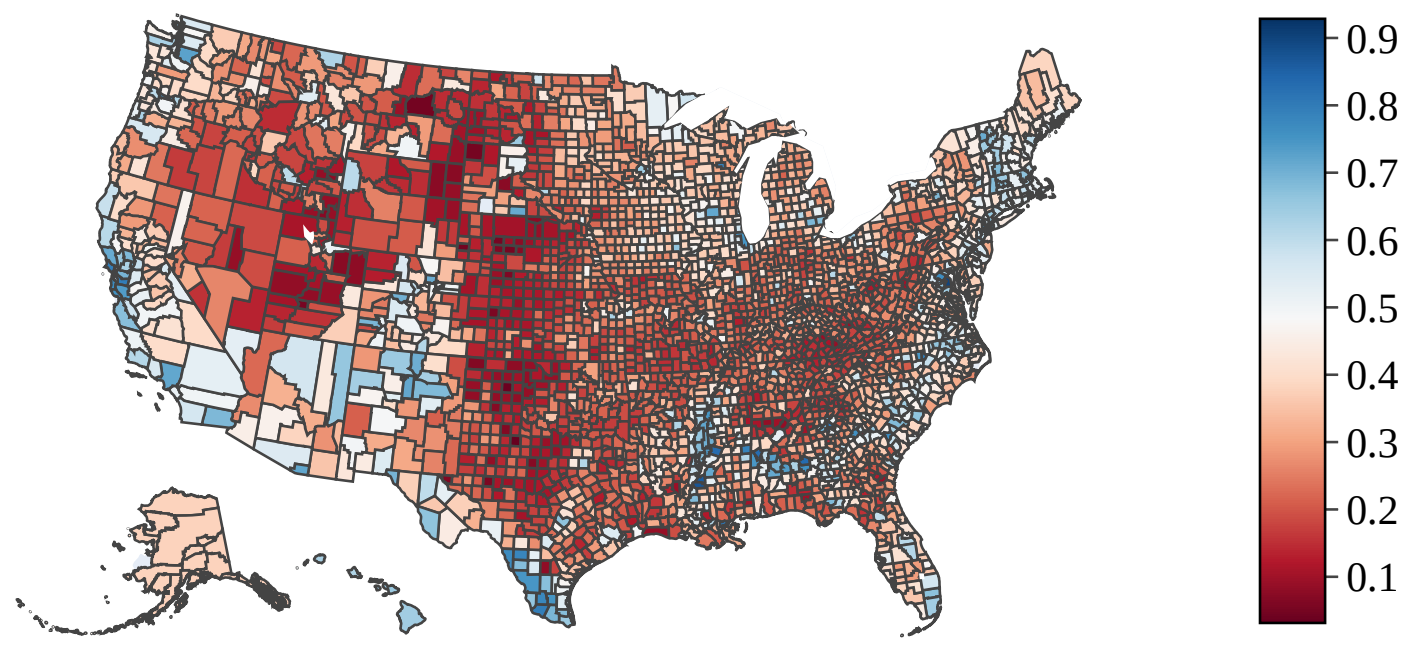

Figure 10.6: Election data. Counties colored by the fraction of people voting for the Democratic candidate in the 2016 election, from red $(0 \%)$ to blue $(100 \%)$. (The urban blue counties are far more densely populated than the rural red counties; in total there were more Democratic than Republican votes.) 

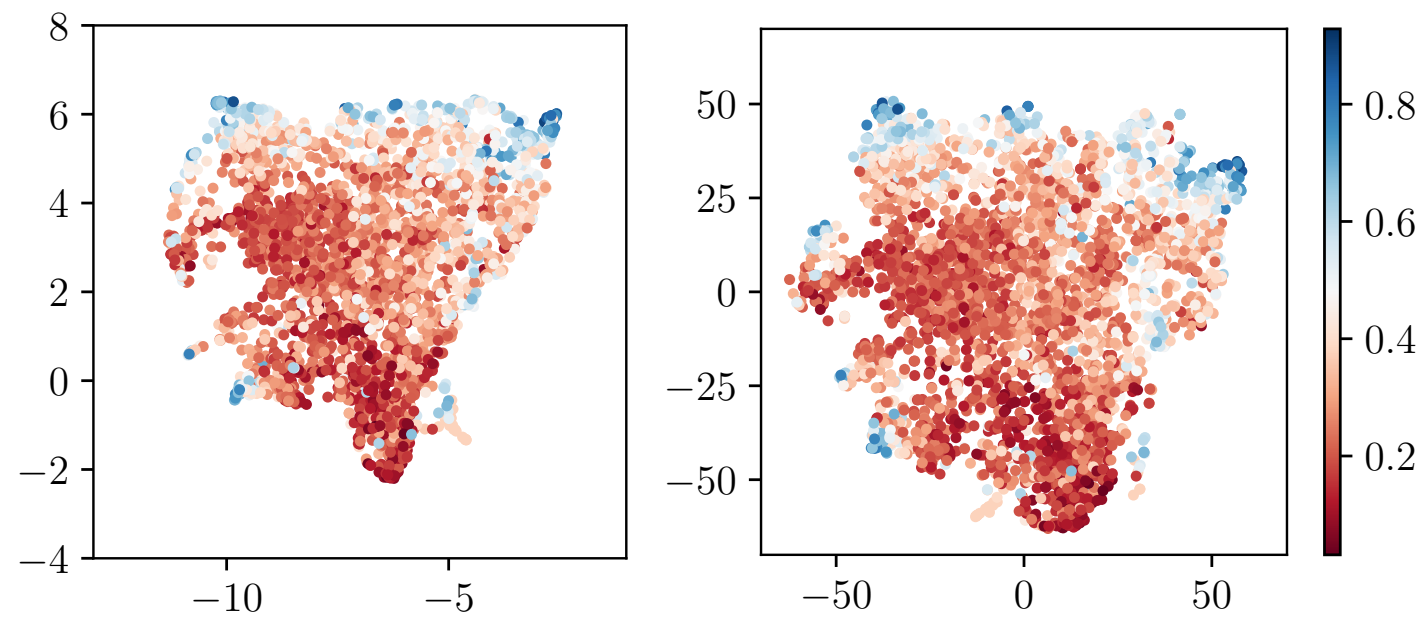

Figure 10.7: UMAP (left) and t-SNE (right) embeddings of the ACS data.

\subsubsection{Comparison to other methods}

We compare the embeddings generated by UMAP and t-SNE, shown in figure 10.7. To make it easier to compare the embeddings, we orthogonally transformed these two embeddings with respect to the unconstrained embedding (as described in §2.4.5), before plotting them. The embeddings broadly resemble the unconstrained embedding, though they appear to be a little bit more dispersed. 


\section{Chapter 11}

\section{Population Genetics}

Our next example is from population genetics. We consider the well-known study carried out by Novembre et al. $\left[\mathrm{Nov}^{+} 08\right]$ of high-dimensional single nucleotide polymorphism (SNP) data associated with 3,000 individuals thought to be of European ancestry. After carefully cleaning the data in order to remove outliers, the authors embed the data into $\mathbf{R}^{2}$ via PCA, obtaining the embedding shown in figure 11.1. Interestingly, the embedding bears a striking resemblance to the map of Europe, shown in figure 11.2, suggesting a close relationship between geography and genetic variation. (In these figures we use the same color legend used in the original Novembre et al. paper.) This resemblance does not emerge if the outliers are not first removed from the original data.

Follow-on work by Diakonikolas et al. [Dia ${ }^{+} 17$ ] proposed a method for robust dimensionality reduction, showing good results on a version of the data from the original Novembre et al. study that was intentionally corrupted with some bad data. After appropriately tuning a number of parameters, they were able to recover the resemblance to a map of Europe despite the bad data. We will explore this issue of robustness to poor data using distortion functions that are robust, i.e., allow for some pairs of similar items to have large distance.

\subsection{Data}

We create two sets of data: one clean, and one intentionally corrupted, following $\left[\mathrm{Dia}^{+} 17\right]$. We follow the same experiment setup as in $\left[\mathrm{Nov}^{+} 08\right.$; $\left.\mathrm{Dia}^{+} 17\right]$, and work with the SNP data associated with 3,000 individuals thought to be of European ancestry, coming from the Population Reference Sample project [ $\left.\mathrm{Nel}^{+} 08\right]$. Novembre et al. and Diakonikolas et al. both start off by pruning the original data set down 


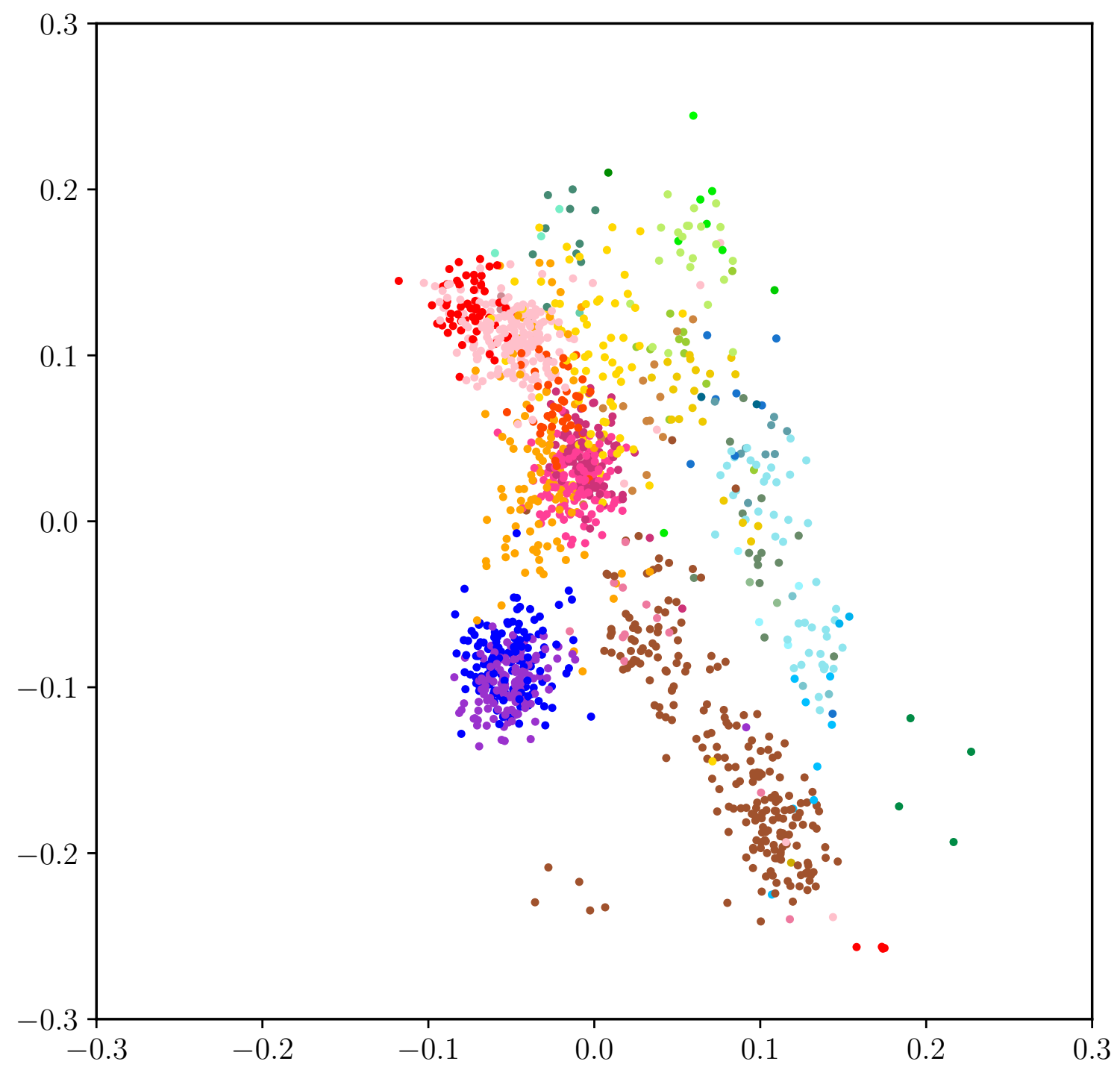

Figure 11.1: PCA embedding of population genetics data. Points are colored by country using the legend shown in the map of Europe in figure 11.2. 


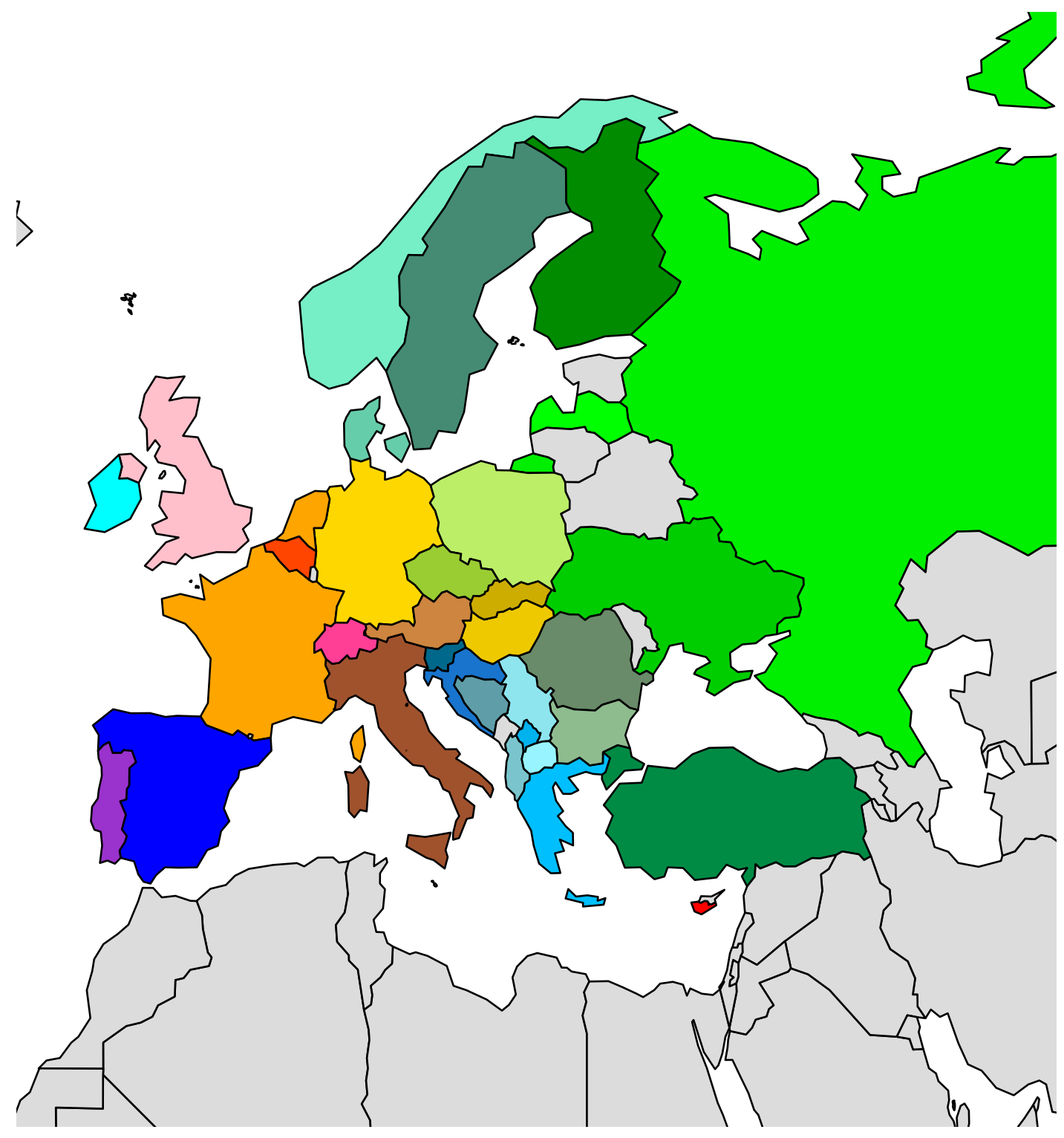

Figure 11.2: Map of Europe, showing the country color legend for the population genetics data, taken from Novembre et al.; countries with no data points are shown in gray. 
to the 1,387 individuals most likely to have European ancestry (explained in the supplementary material of $\left.\left[\mathrm{Nov}^{+} 08\right]\right)$, and then project the data onto its top 20 principal components, obtaining the (clean) samples $y_{i} \in \mathbf{R}^{20}, i=1, \ldots, 1387$.

To create a corrupted set of data, we follow the method of Diakonikolas et al., who randomly rotate the data and then inject 154 additional points $y_{i}, i=1388, \ldots, 1541$, where the first ten entries of the $y_{i}$ are i.i.d. following a discrete uniform distribution on $\{0,1,2\}$, and the last ten entries of the $y_{i}$ are i.i.d. following a discrete uniform distribution on $\{1 / 12,1 / 8\}$. Thus the clean data is $y_{1}, \ldots, y_{1387}$, and the corrupted data is $y_{1388}, \ldots, y_{1541}$.

We also have the country of origin for each of the data points, given as one of 34 European countries. We use this attribute to check our embeddings, but not to construct our embeddings. In plots, we color the synthesized (corrupted) data points black.

\subsection{Preprocessing}

For both the clean and corrupted raw data sets we construct a set of pairs of similar people $\mathcal{E}_{\text {sim }}$ as the $k$-nearest (Euclidean) neighbors, with $k=15$. For the clean data, we have $\left|\mathcal{E}_{\text {sim }}\right|=16,375$, and for the corrupted data we have $\left|\mathcal{E}_{\text {sim }}\right|=17,842$. We also treat the remaining pairs of people as dissimilar, so that $\left|\mathcal{E}_{\text {dis }}\right|=944,816$ for the clean data, and $\left|\mathcal{E}_{\text {dis }}\right|=1,168,728$ for the corrupted data. We use weights $w_{i j}=2$ for $(i, j) \in \mathcal{E}_{\text {sim }}$ when individuals $i$ and $j$ are both neighbors of each other. We use weights $w_{i j}=1$ for $(i, j) \in \mathcal{E}_{\text {sim }}$ when $i$ is a neighbor of $j$ but $j$ is not a neighbor of $i$ (or vice versa). We use weights $w_{i j}=-1$ for $(i, j) \in \mathcal{E}_{\text {dis }}$.

\subsection{Embedding}

\subsubsection{PCA embedding}

We start with PCA, exactly following Novembre et al., which on the clean data results in the embedding shown in figure 11.1. In the language of this monograph, this embedding uses quadratic distortion for $(i, j) \in \mathcal{E}$, weights $w_{i j}=y_{i}^{T} y_{j}$ (after centering the data), and a standardization constraint. The distribution of the weights is shown in figure 11.4. When PCA embedding is used on the corrupted data, we obtain the embedding in figure 11.3. In this embedding only some of the resemblance to the map of Europe is preserved. The synthesized points are embedded on the right 
side. (If those points are manually removed, and we PCA run again, we recover the original embedding.)

\subsubsection{Unconstrained embeddings}

We embed both the clean and corrupted data unconstrained (centered), based on the graph $\mathcal{E}=\mathcal{E}_{\text {sim }} \cup \mathcal{E}_{\text {dis }}$. We use a Huber attractive penalty (4.2), and a logarithmic repulsive penalty $(4.4)(\alpha=1)$ to enforce spreading. The embedding of the clean data is shown in figure 11.5, and for the corrupted data in figure 11.6. We can see that this embedding is able to recover the similarity to the map of Europe, despite the corrupted data. In fact, this embedding appears virtually identical to the embedding of the clean data in figure 11.5.

\subsubsection{Comparison to other methods}

We show the UMAP and t-SNE embeddings on the clean and corrupted data in figure 11.7. These embeddings were scaled and orthogonally transformed with respect to the map given in figure 11.2, following the method described in $\S 2.4 .5$. The embeddings with the corrupted data are better than the PCA embedding. 


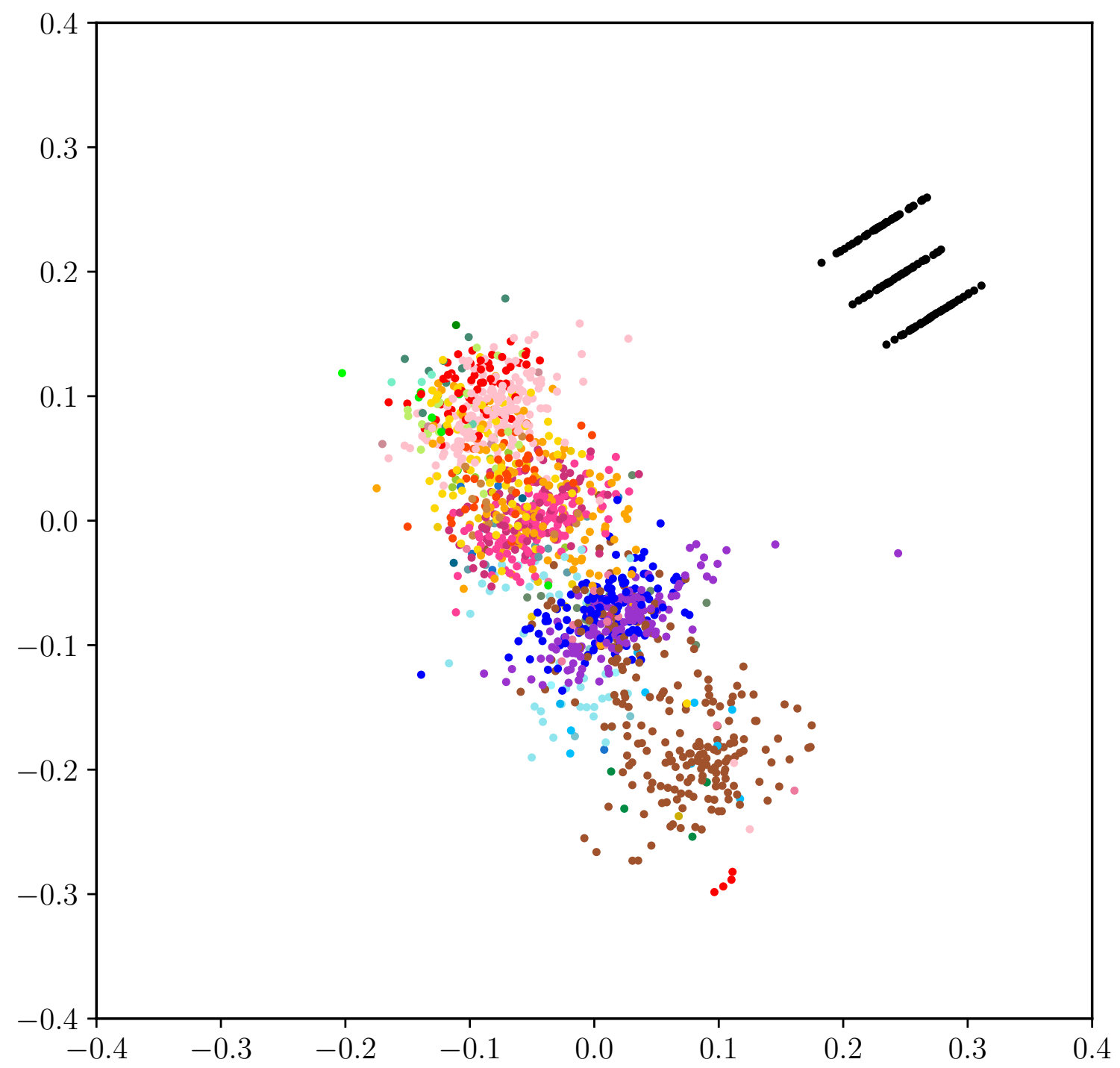

Figure 11.3: PCA embedding of corrupted data. The resemblance to a map of Europe is substantially reduced. 

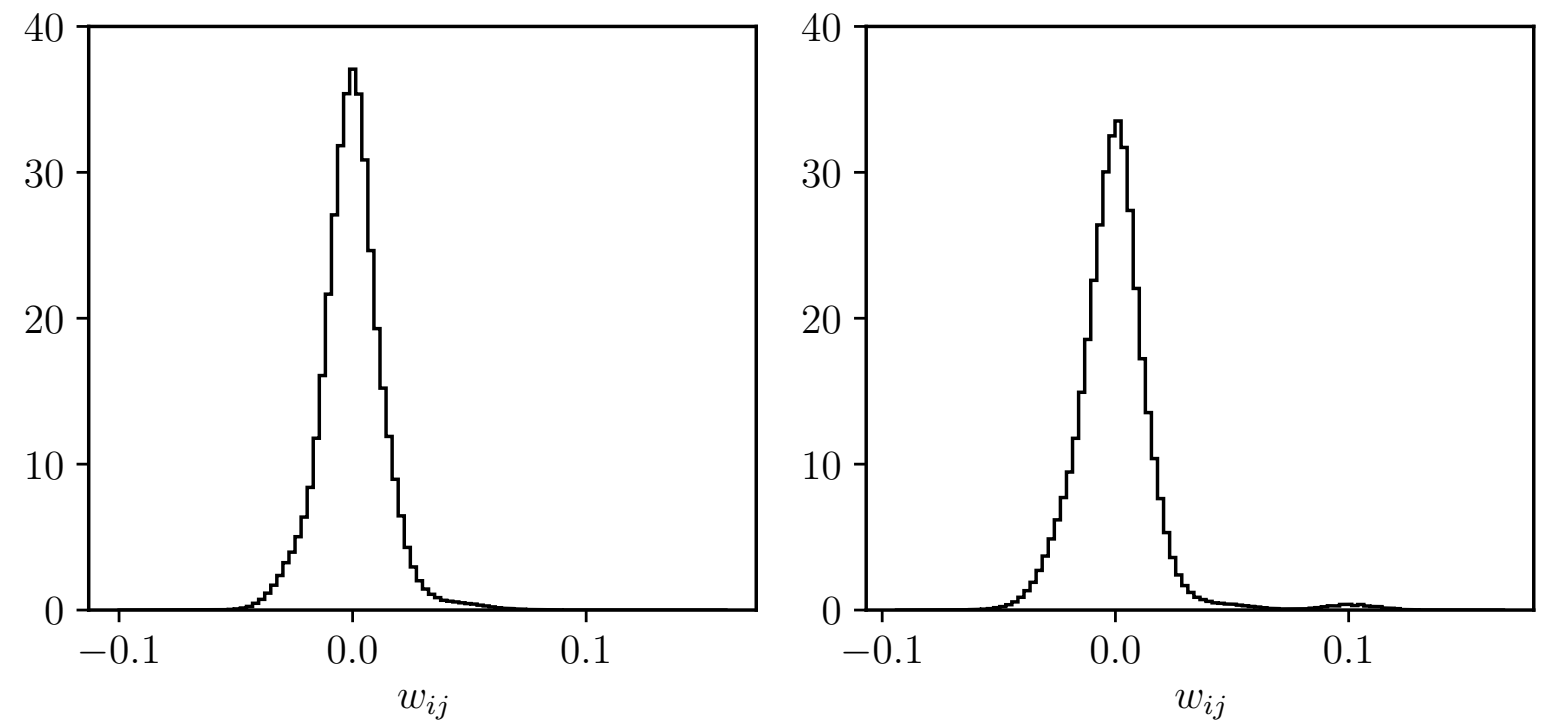

Figure 11.4: PCA weights. Distribution of the weights used by PCA, when expressed as an MDE problem, for the clean (left) and corrupted (right) data. 


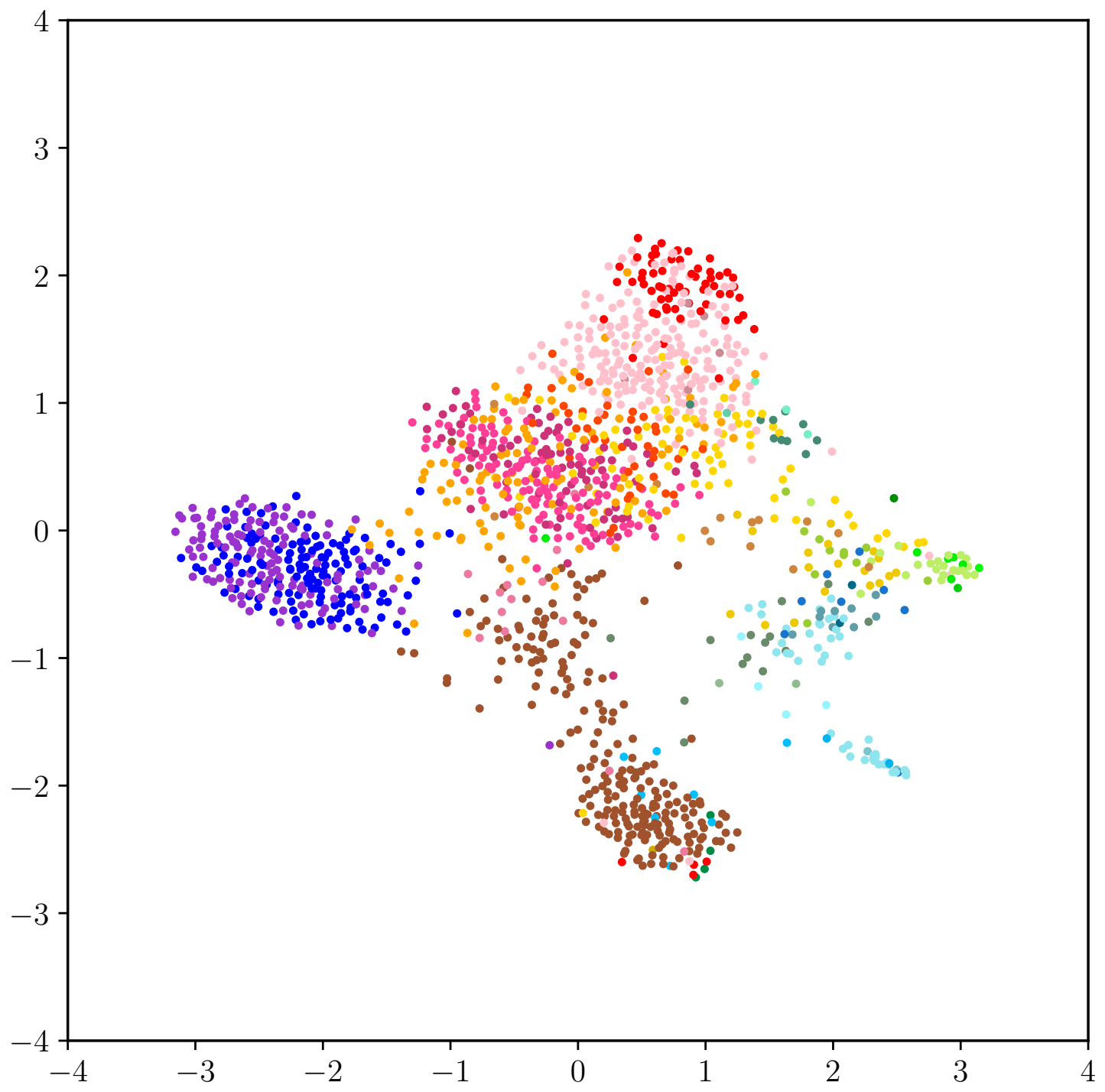

Figure 11.5: Embedding of clean data. The embedding resembles a map of Europe. 


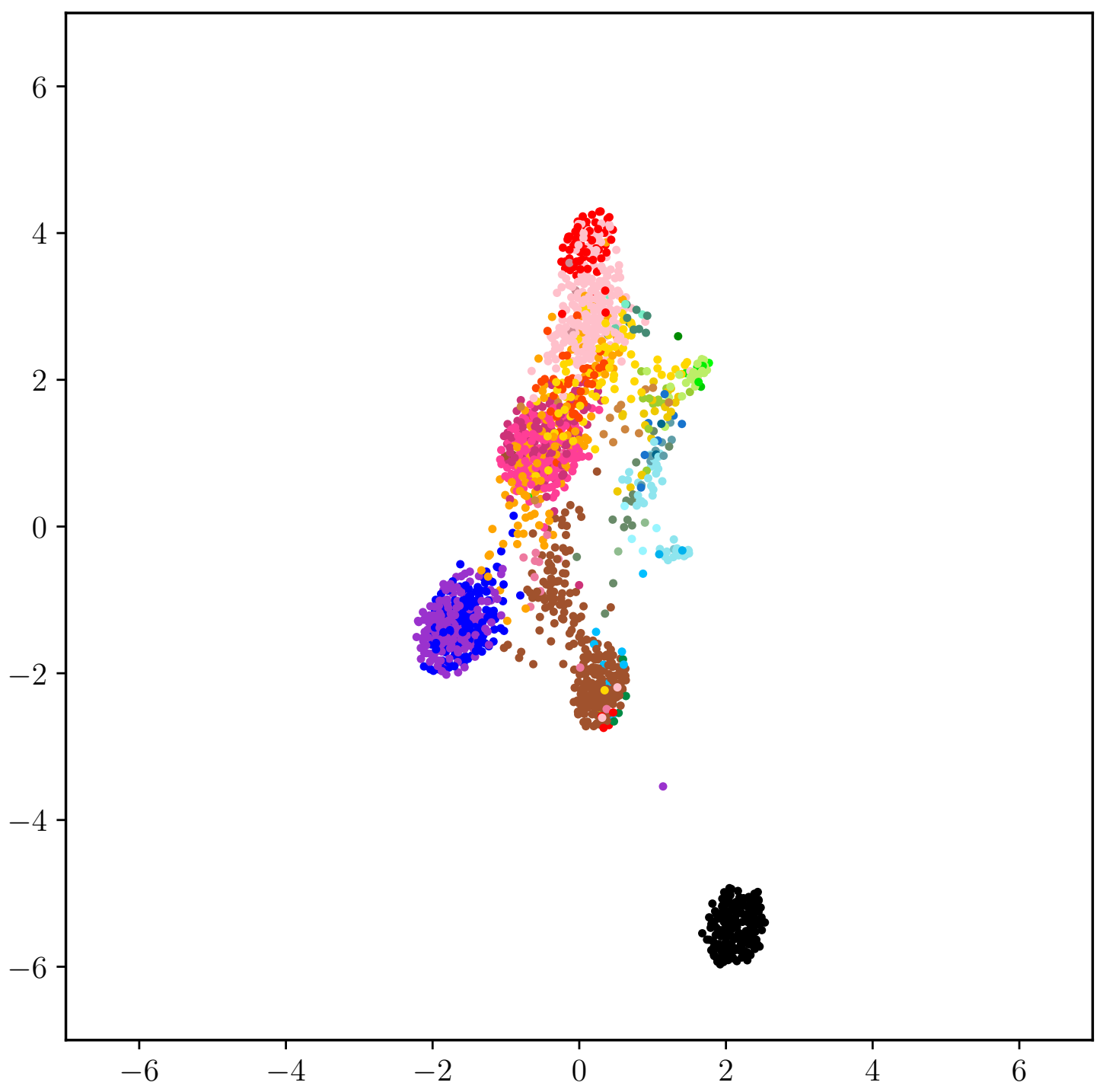

Figure 11.6: Embedding of corrupted data. The embedding is able to recover the similarity to the map of Europe. 

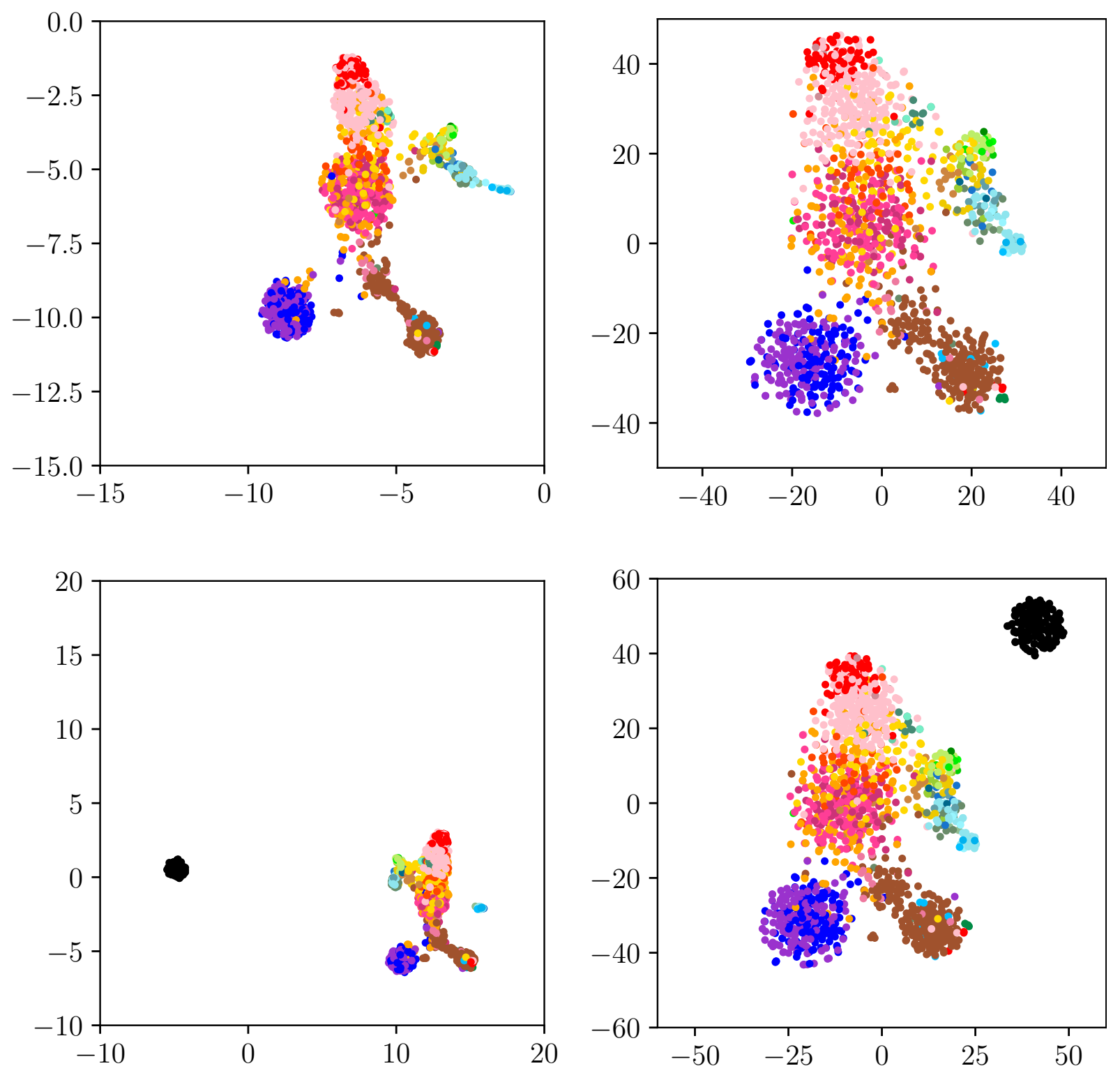

Figure 11.7: UMAP (left) and t-SNE (right) embeddings of clean (top) and corrupted data (bottom). 


\section{Chapter 12}

\section{Single-Cell Genomics}

For our last example we embed several thousand single-cell mRNA (scRNA) transcriptomes. We use data that accompanies the recent publication [Wil $\left.{ }^{+} 20\right]$; these data are sequences of human peripheral blood mononuclear cells (PBMCs), collected from seven patients afflicted with severe COVID-19 infections and six healthy controls. We embed into $\mathbf{R}^{3}$ and visualize the embedding vectors, finding that the vectors are organized by donor health status and cell type.

\subsection{Data}

The dataset from [Wil $\left.{ }^{+} 20\right]$ includes $n=44,721$ scRNA transcriptomes of PBMCs, with 16,627 cells from healthy donors and 28,094 from donors infected with COVID19. Each cell is represented by a sparse vector in $\mathbf{R}^{26361}$, and each component gives the expression level of a gene. The authors of $\left[\mathrm{Wil}^{+} 20\right]$ projected the data matrix onto its top 50 principal components, yielding a matrix $Y \in \mathbf{R}^{44721 \times 50}$ which they further studied.

In constructing our embedding, we use only the reduced gene expression data, i.e., the entries of $Y$. Each cell is tagged with many held-out attributes, and we use two of these to informally validate our embedding. The first attribute says whether a cell came from a donor infected with COVID-19, and the second gives a classification of the cells into (manually labeled) sub-families. 


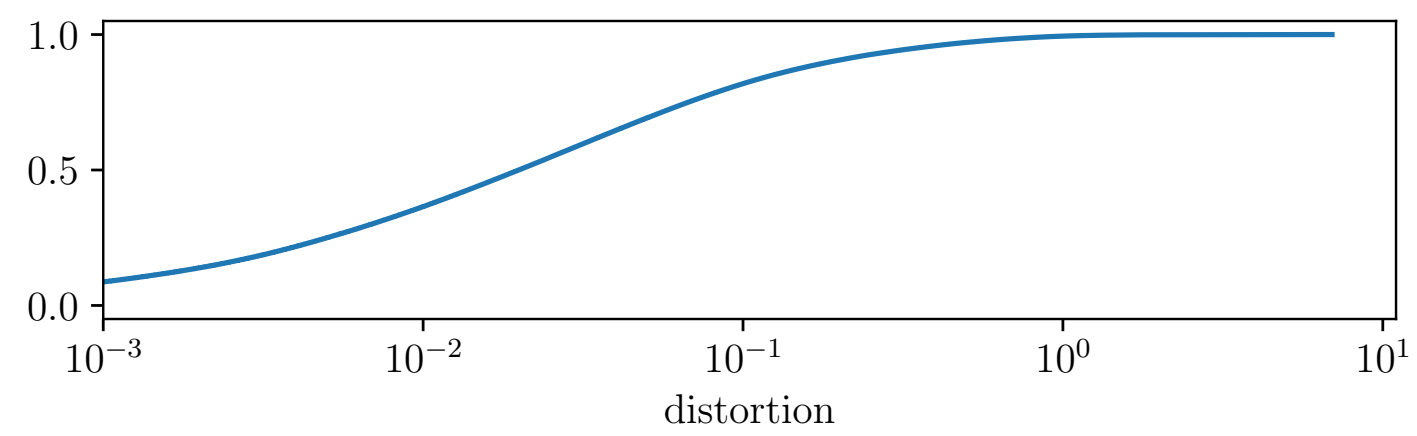

Figure 12.1: CDF of distortions for the embedding of scRNA data.

\subsection{Preprocessing}

We use distortion functions derived from weights. From the rows of $Y$ we construct a $k$-nearest (Euclidean) neighbor graph, with $k=15$; this gives a graph $\mathcal{E}_{\text {sim }}$ with 515,376 edges. We then sample, uniformly at random, an additional $\left|\mathcal{E}_{\text {sim }}\right|$ pairs not in $\mathcal{E}_{\text {sim }}$ to obtain a graph $\mathcal{E}_{\text {dis }}$ of dissimilar cells. The final graph $\mathcal{E}=\mathcal{E}_{\text {sim }} \cup \mathcal{E}_{\text {dis }}$ has $p=1,030,752$ edges. For the weights, we choose $w_{i j}=+2$ if $i$ and $j$ are both neighbors of each other; $w_{i j}=+1$ if $i$ is a neighbor of $j$ but $j$ is not a neighbor of $i$ (or vice versa); and $w_{i j}=-1$ for $(i, j) \in \mathcal{E}_{\text {dis }}$.

\subsection{Embedding}

We form a standardized MDE problem with embedding dimension $m=3$, based on the graph $\mathcal{E}=\mathcal{E}_{\text {sim }} \cup \mathcal{E}_{\text {dis }}$. We use the log-one-plus penalty (4.3) ( $\left.\alpha=1.5\right)$ for $p_{\mathrm{s}}$ and the logarithmic penalty (4.4) $(\alpha=1)$ for $p_{\mathrm{d}}$.

We solved the MDE problem with PyMDE, initializing the solve with a embedding obtained by solving a quadratic MDE problem on $\mathcal{E}_{\text {sim. }}$. The quadratic MDE problem was solved in 7 seconds (249 iterations) on our CPU, and 2 seconds (185 iterations) on our GPU. Solving the MDE problem took 27 seconds (300 iterations) on our CPU, and 6 seconds (300 iterations) on our GPU. The embedding has average distortion 0.13; a CDF of distortions is shown in figure 12.1. The embedding used for initialization is shown in figure 12.2, with embedding vectors colored by the cell-type attribute.

The embedding is shown in figure 12.3, colored by cell type; similar cells end up near each other in the embedding. The embedding is plotted again in figure 12.4, 
this time colored by donor health status. Cells from healthy donors are nearer to each other than to cells from infected donors, and the analogous statement is true for cells from infected donors.

As a sanity check, we partition $\mathcal{E}_{\text {sim }}$ into three sets: edges between healthy donors, edges between infected patients, and edges between infected patients and healthy donors. The average distortion of the embedding, restricted to each of these sets, is $0.19,0.18$, and 0.21 , respectively. This means pairs linking healthy donors to infected donors are more heavily distorted on average than pairs linking donors with the same health status.

Figure 12.5 shows the embedding with roughly 10 percent of the pairs in $\mathcal{E}_{\text {sim }}$ overlaid as white line segments (the pairs were sampled uniformly at random). Cells near each other are highly connected to each other, while distant cells are not. Finally, the 1000 pairs from $\mathcal{E}_{\text {sim }}$ with the highest distortions are shown in figure 12.6; most of these pairs contain different types of cells. 

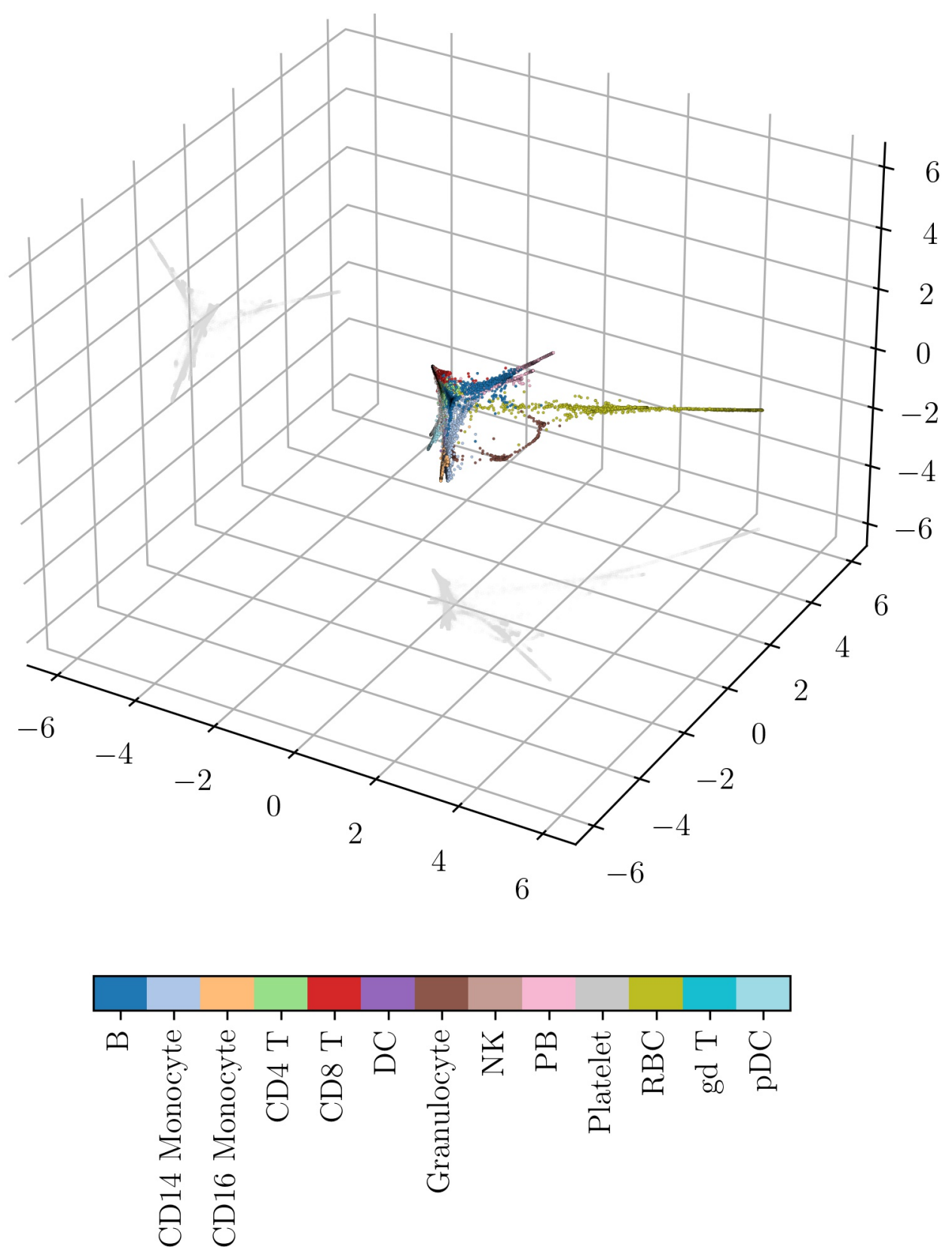

Figure 12.2: Embedding based on $\mathcal{E}_{\text {sim. }}$. An embedding of scRNA transcriptomes of PBMCs from patients with severe COVID-19 infections and healthy controls $\left[\mathrm{Wil}^{+} 20\right]$, obtained by solving a quadratic MDE problem on $\mathcal{E}_{\text {sim }}$ and colored by cell type. 

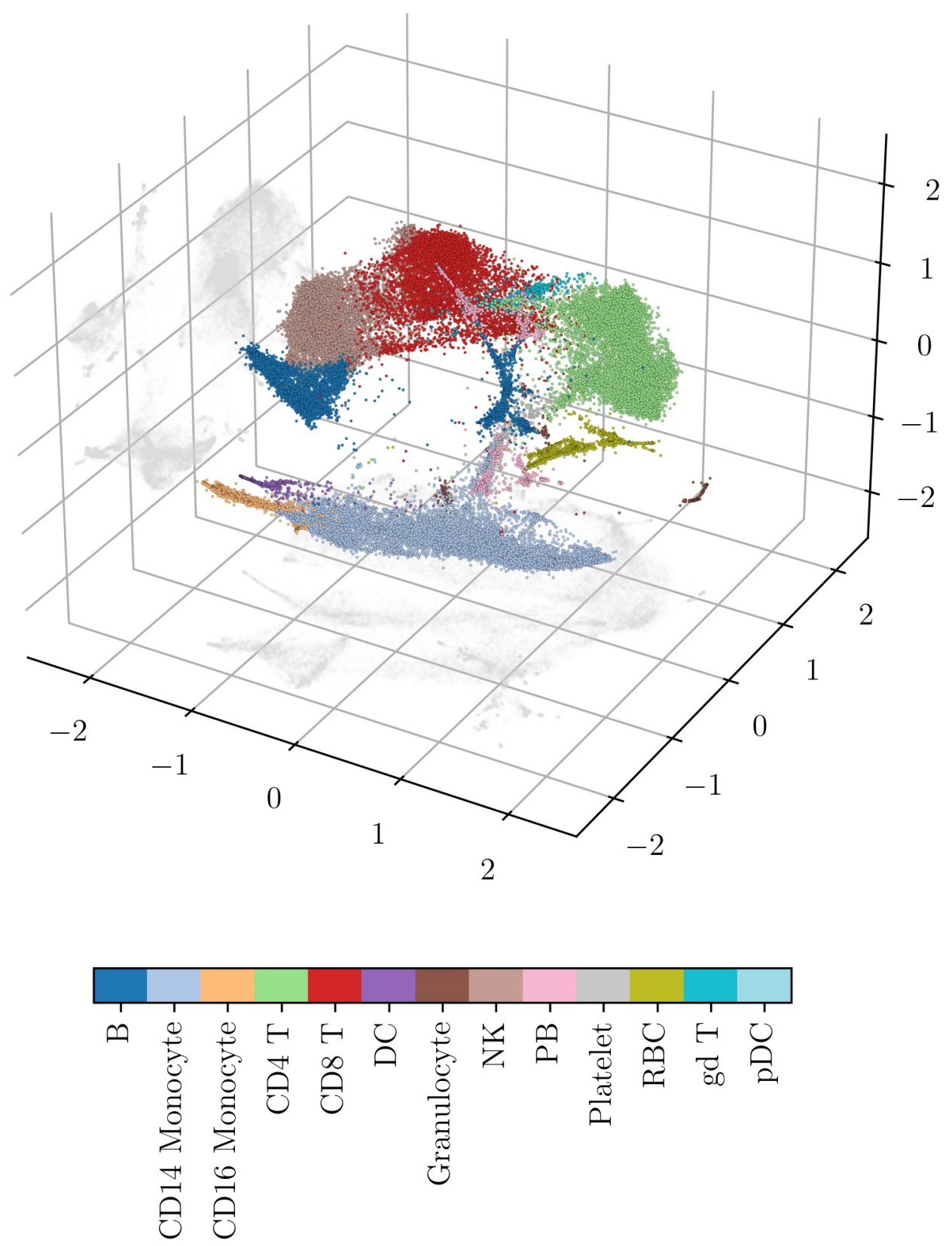

Figure 12.3: Embedding based on $\mathcal{E}_{\text {sim }} \cup \mathcal{E}_{\text {dis }}$. Embedding of scRNA transcriptomes, colored by cell type. 

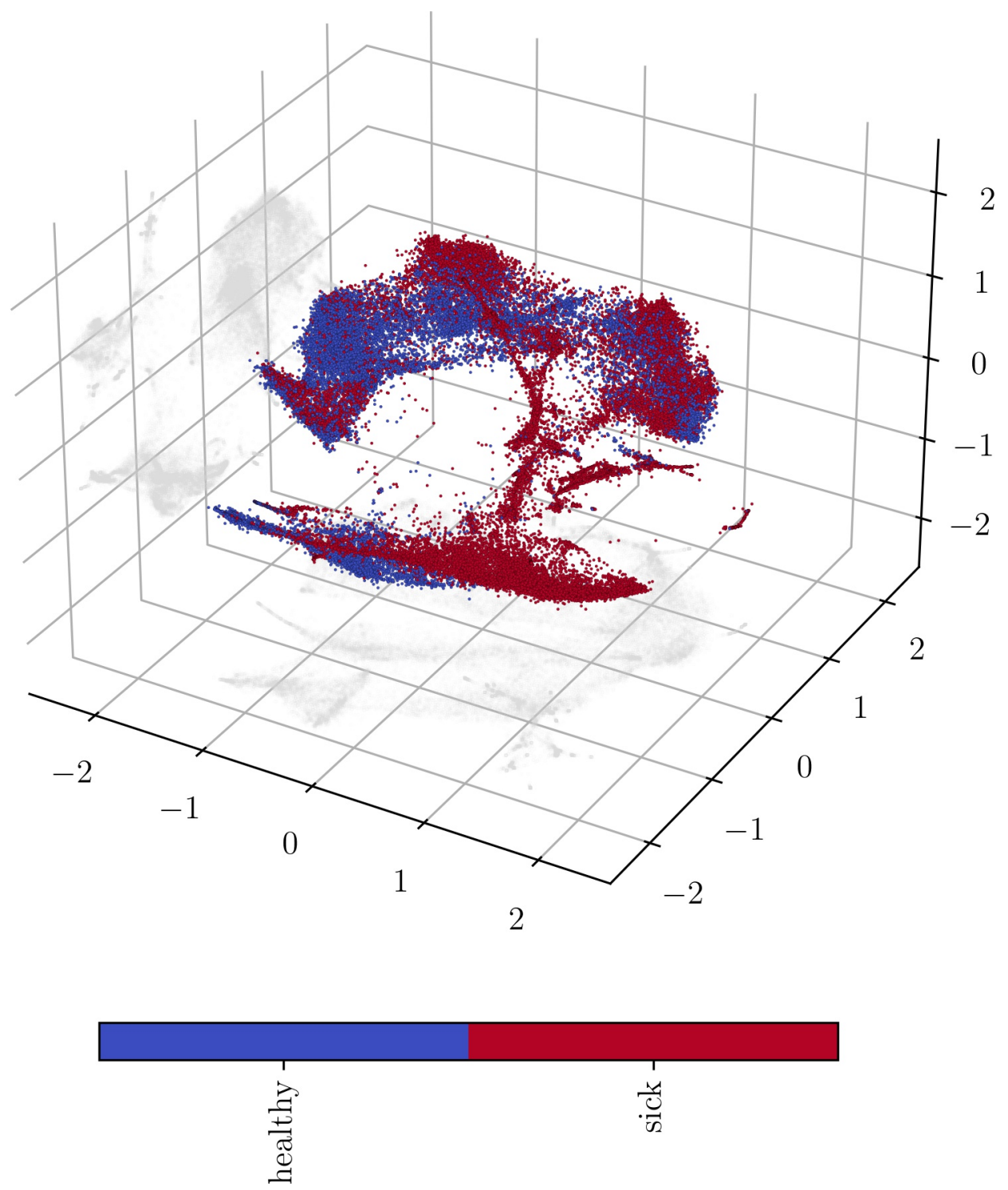

Figure 12.4: Health status. Embedding of scRNA data, colored by health status. 

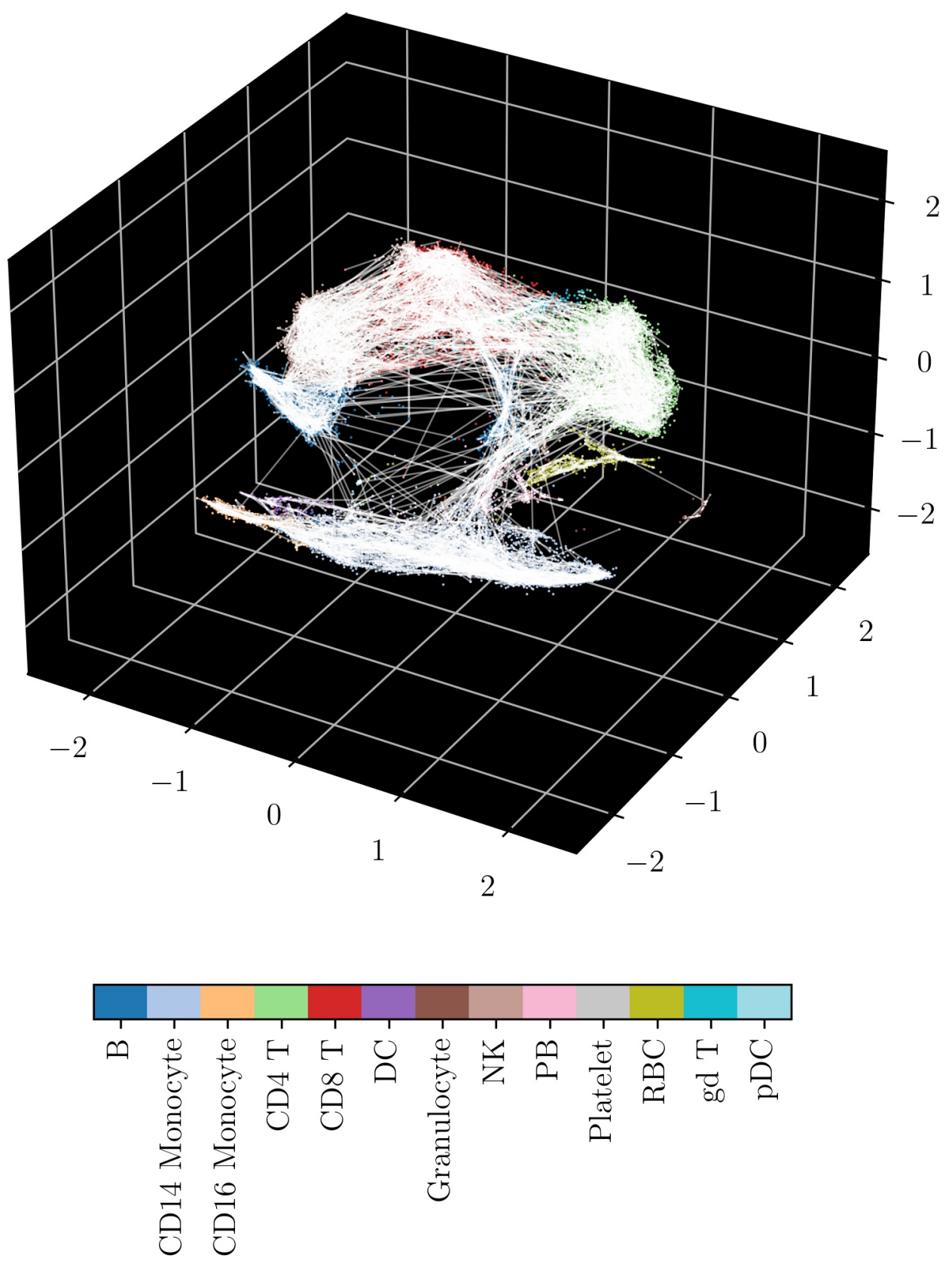

Figure 12.5: With edges. Embedding of scRNA data, with edges between neighboring cells displayed as white line segments. 

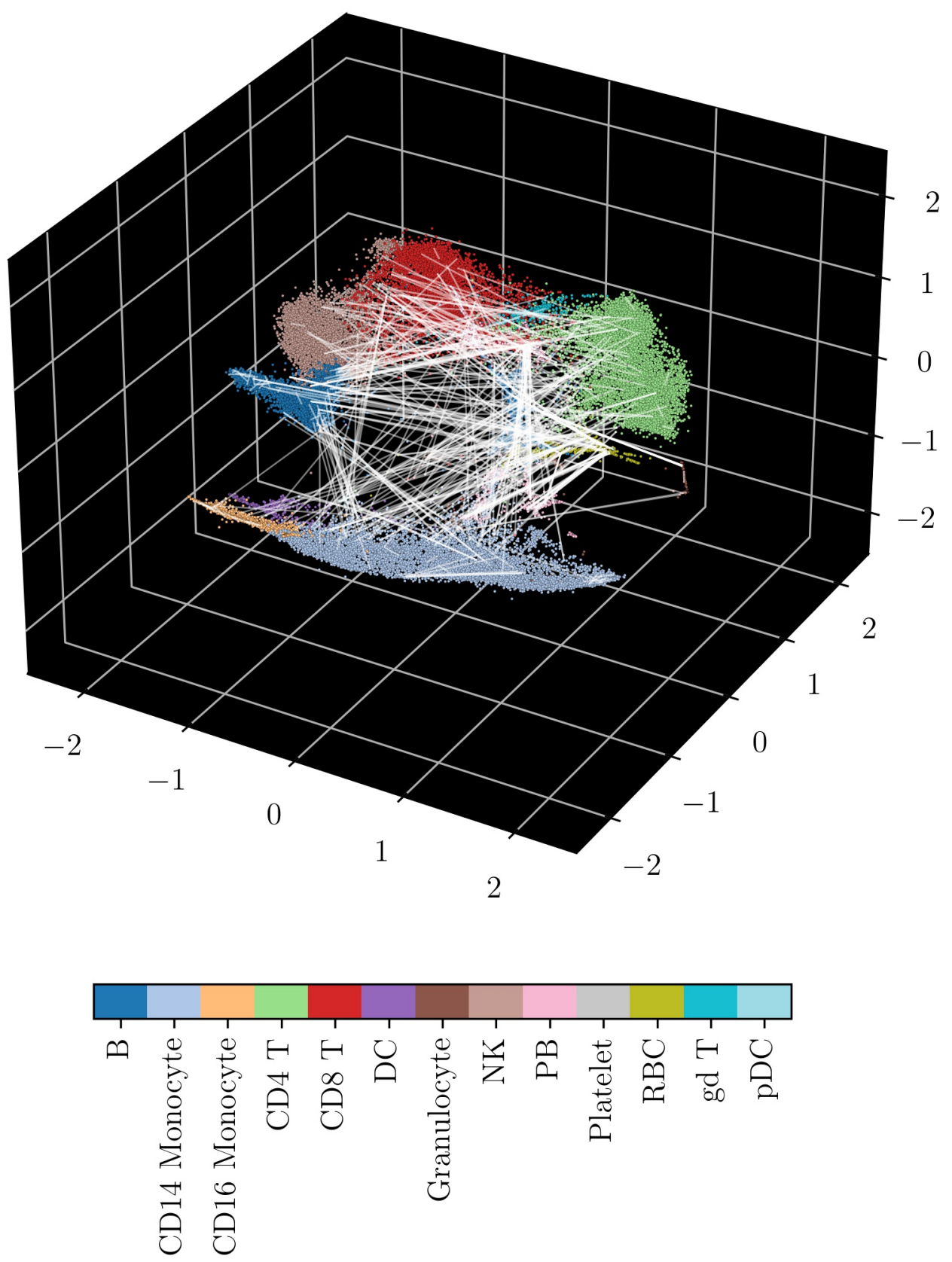

Figure 12.6: High distortion pairs. Embedding of scRNA data, with the 1000 most heavily distorted pairs displayed as white line segments. 


\subsubsection{Comparison to other methods}

Figure 12.7 shows an embedding of the scRNA data produced by UMAP, which took 11 seconds, and figure 12.8 shows an embedding made using openTSNE, which took 96 seconds, using the value bh for the parameter negative_gradient_method (the default value resulted in an error). The embeddings were aligned to the orientation of our embedding by solving a Procrustes problem, as described in $§ 2.4 .5$. 

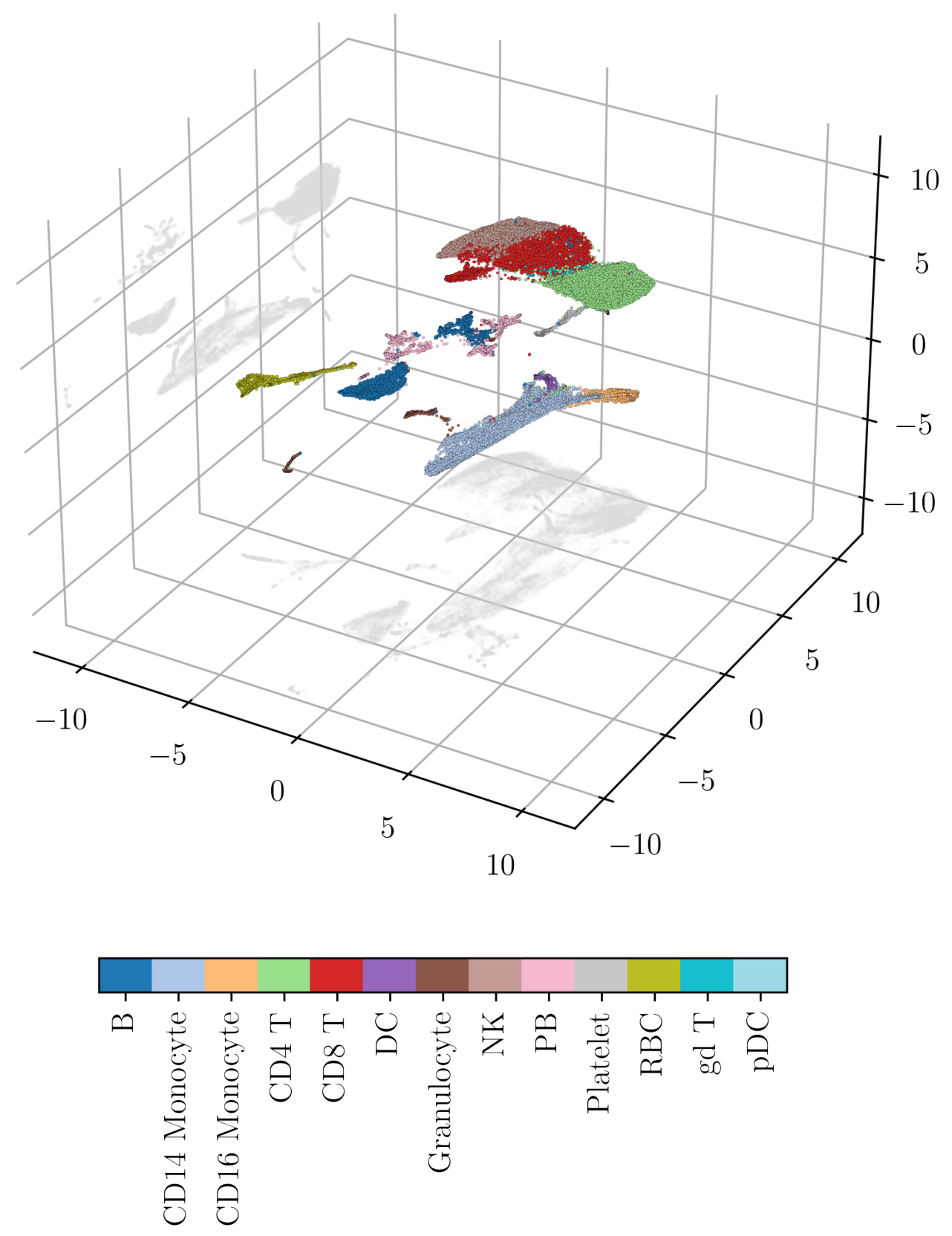

Figure 12.7: UMAP. Embedding of scRNA data, produced by UMAP. 

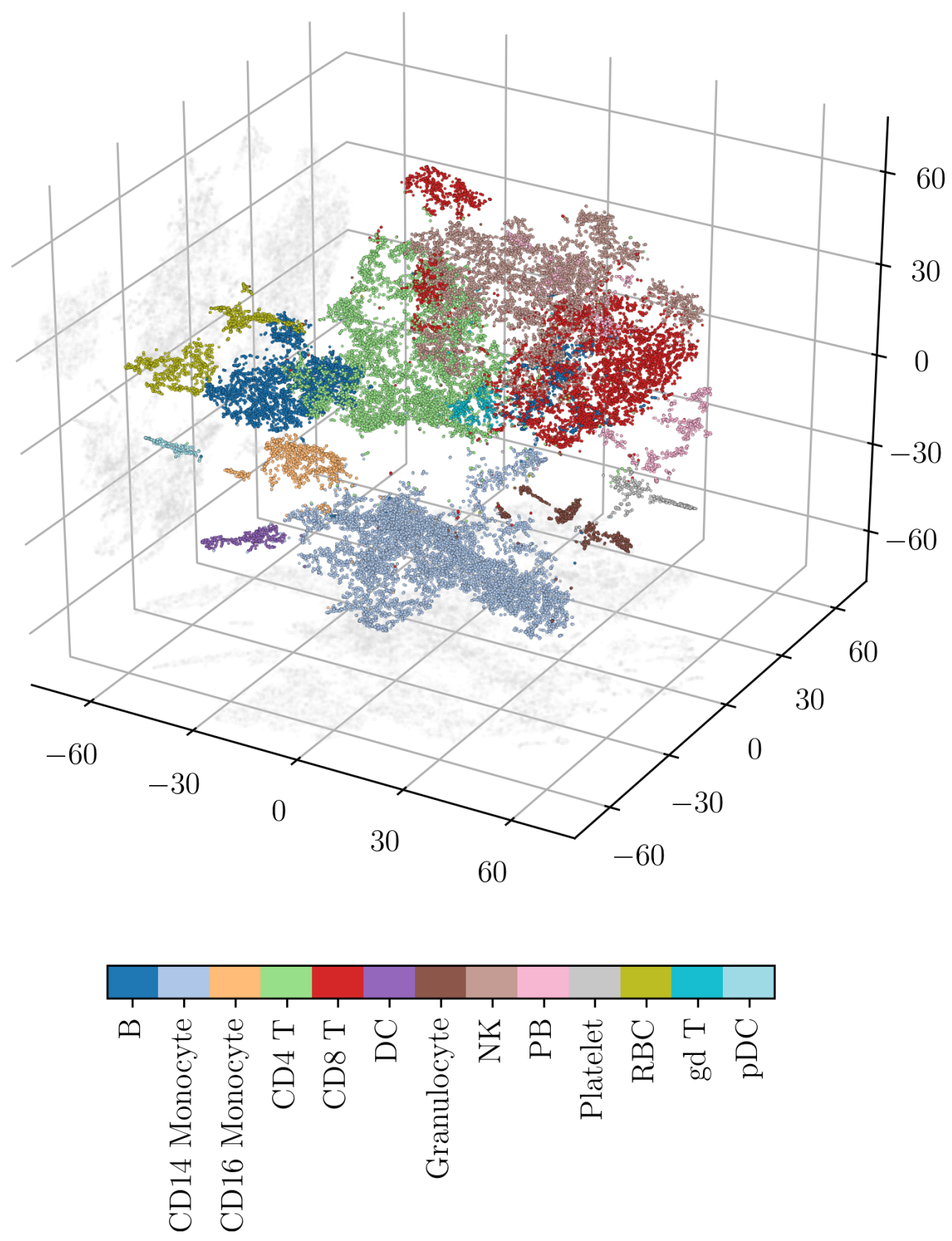

Figure 12.8: $t$-SNE. Embedding of scRNA data, produced by t-SNE. 


\section{Chapter 13}

\section{Conclusions}

The task of vector embedding, i.e., assigning each of a set of items an associated vector, is an old and well-established problem, with many methods proposed over the past 100 years. In this monograph we have formalized the problem as one of choosing the vectors so as to minimize an average or total distortion, where distortion functions specified for pairs of items express our preferences about the Euclidean distance between the associated vectors. Roughly speaking, we want vectors associated with similar items to be near each other, and vectors associated with dissimilar items to not be near each other.

The distortion functions, which express our prior knowledge about similarity and dissimilarity of pairs of items, can be constructed in several different but related ways. Weights on edges give us a measure of similarity (when positive) and dissimilarity (when negative). Alternatively, we can start with deviations between pairs of items, with small deviation meaning high similarity. Similarity and dissimilarity can also be expressed by one or more graphs on the items, for example one specifying similar pairs and another specifying dissimilar pairs. There is some art in making the distortion functions, though we find that a few simple preprocessing steps, such as the ones described in this monograph, work well in practice.

Our framework includes a large number of well-known previously developed methods as special cases, including PCA, Laplacian embedding, UMAP, multi-dimensional scaling, and many others. Some other existing embedding methods cannot be represented as MDE problems, but MDE-based methods can produce similar embeddings. Our framework can also be used to create new types of embeddings, depending on the choice of distortion functions, the constraint set, and the preprocessing of original data.

The quality of an embedding ultimately depends on whether it is suitable for the 
downstream application. Nonetheless we can use our framework to validate, or at least sanity-check, embeddings. For example, we can examine pairs with abnormally high distortion. We can then see if these pairs contain anomalous items, or whether their distortion functions inaccurately conveyed their similarity or lack thereof.

Our examples in part III focused on embedding into two or three dimensions. This allowed us to plot the embeddings, which we judged by whether they led to insight into the data, as well as by aesthetics. But we can just as well embed into dimensions larger than two or three, as might be done when developing a feature mapping on the items for downstream machine learning tasks. When the original data records are high-dimensional vectors (say, several thousand dimensions), we can embed them into five or ten dimensions and use the embedding vectors as the features; this makes the vector representation of the data records much smaller, and the fitting problem more tractable. It can also improve the machine learning, since the embedding depends on whatever data we used to carry it out, and in some sense inherits its structure.

It is practical to exactly solve MDE problems only in some special cases, such as when the distortion functions are quadratic and the embedding vectors are required to be standardized. For other cases, we have introduced an efficient local optimization method that produces good embeddings, while placing few assumptions on the distortion functions and constraint set. We have shown in particular how to reliably compute embeddings with a standardization constraint, even when the objective function is not quadratic.

Our optimization method (and software) scales to very large problems, with embedding dimensions much greater than two or three, and our experiments show that it is competitive in runtime to more specialized algorithms for specific embedding methods. The framework of MDE problems, coupled with our solution method and software, makes it possible for practitioners to rapidly experiment with new kinds of embeddings in a principled way, without sacrificing performance. 


\section{Acknowledgments}

We thank Amr Alexandari, Guillermo Angeris, Shane Barratt, Steven Diamond, Mihail Eric, Andrew Knyazev, Benoit Rostykus, Sabera Talukder, Jian Tang, Jonathan Tuck, Junzi Zhang, as well as several anonymous reviewers, for their comments on the manuscript; Yifan Lu and Lawrence Saul, for their careful readings and many thoughtful suggestions; and Delenn Chin and Dmitry Kobak, for their detailed feedback on both the manuscript and early versions of the software package. 


\section{References}

[AM12] P.-A. Absil and J. Malick. Projection-like retractions on matrix manifolds. SIAM Journal on Optimization 22.1 (2012), pp. 135-158.

[AMS09] P.-A. Absil, R. Mahony, and R. Sepulchre. Optimization Algorithms on Matrix Manifolds. Princeton University Press, 2009.

$\left[\mathrm{Ahm}^{+} 20\right] \quad$ N. Ahmed, R. Rossi, J. Lee, T. Willke, R. Zhou, X. Kong, and H. Eldardiry. Role-based graph embeddings. IEEE Transactions on Knowledge and Data Engineering (2020).

[Alc16] M. Alcorn. (batter-pitcher)2vec: Statistic-free talent modeling with neural player embeddings. In. MIT Sloan Sports Analytics Conference. 2016.

[AIR18] A. Andoni, P. Indyk, and I. Razenshteyn. Approximate nearest neighbor search in high dimensions. arXiv (2018).

[Arr50] K. Arrow. A difficulty in the concept of social welfare. Journal of Political Economy 58.4 (1950), pp. 328-346.

[AM15] E. Asgari and M. Mofrad. Continuous distributed representation of biological sequences for deep proteomics and genomics. PLOS One 10.11 (2015), pp. 1-15.

[AD19] H. Asi and J. Duchi. Stochastic (approximate) proximal point methods: Convergence, optimality, and adaptivity. SIAM Journal on Optimization 29.3 (2019), pp. 2257-2290.

[BHN18] S. Barocas, M. Hardt, and A. Narayanan. Fairness and Machine Learning: Limitations and Opportunities. 2018.

[BG97] R. Beatson and L. Greengard. A short course on fast multipole methods. In Wavelets, Multilevel Methods and Elliptic PDEs. Oxford University Press, 1997, pp. 1-37. 
[BN02] M. Belkin and P. Niyogi. Laplacian eigenmaps and spectral techniques for embedding and clustering. In Advances in Neural Information Processing Systems. 2002, pp. 585-591.

$\left[\right.$ Ben $\left.^{+} 21\right]$ E. Bender, T. Gebru, A. McMillan-Major, and S. Shmitchell. On the dangers of stochastic parrots: Can language models be too big? In Proceedings of the 2021 Conference on Fairness, Accountability, and Transparency. 2021.

[Ber20a] R. Bergmann. Manopt.jl. 2020. URL: https://manoptjl.org/stable/ index.html.

[Ber20b] E. Bernhardsson. annoy. 2020. URL: https://github.com/spotify/ annoy.

$\left[\mathrm{Ber}^{+} 00\right] \quad$ M. Bernstein, V. De Silva, J. Langford, and J. Tenenbaum. Graph approximations to geodesics on embedded manifolds. Tech. rep. Department of Psychology, Stanford University, 2000.

[BY04] P. Biswas and Y. Ye. Semidefinite programming for ad hoc wireless sensor network localization. In Proceedings of the 3rd International Symposium on Information Processing in Sensor Networks. 2004, pp. 46-54.

[BBK20] J. N. Böhm, P. Berens, and D. Kobak. A unifying perspective on neighbor embeddings along the attraction-repulsion spectrum. arXiv (2020).

[Bol ${ }^{+}$16] T. Bolukbasi, K.-W. Chang, J. Zou, V. Saligrama, and A. Kalai. Man is to computer programmer as woman is to homemaker? Debiasing word embeddings. In Advances in Neural Information Processing Systems. 2016, 4356-4364.

[BG03] I. Borg and P. Groenen. Modern multidimensional scaling: Theory and applications. Journal of Educational Measurement 40.3 (2003), pp. 277280.

$\left[\mathrm{Bou}^{+} 14\right] \quad$ N. Boumal, B. Mishra, P.-A. Absil, and R. Sepulchre. Manopt, a Matlab toolbox for optimization on manifolds. Journal of Machine Learning Research 15.1 (2014), pp. 1455-1459.

[Bou85] J. Bourgain. On Lipschitz embedding of finite metric spaces in Hilbert space. Israel Journal of Mathematics 52.1-2 (1985), pp. 46-52.

[BV04] S. Boyd and L. Vandenberghe. Convex Optimization. New York, NY, USA: Cambridge University Press, 2004. 
[BV18] S. Boyd and L. Vandenberghe. Introduction to Applied Linear Algebra: Vectors, Matrices, and Least Squares. New York, NY, USA: Cambridge University Press, 2018.

[BT52] R. Bradley and M. Terry. Rank analysis of incomplete block designs: The method of paired comparisons. Biometrika 39.3/4 (1952), pp. 324-345.

[Bro70] C. G. Broyden. The convergence of a class of double-rank minimization algorithms, general considerations. IMA Journal of Applied Mathematics 6.1 (1970), pp. 76-90.

[BM03] S. Burer and R. Monteiro. A nonlinear programming algorithm for solving semidefinite programs via low-rank factorization. Mathematical Programming 95.2 (2003), pp. 329-357.

[BM05] S. Burer and R. Monteiro. Local minima and convergence in low-rank semidefinite programming. Mathematical Programming 103.3, Ser. A (2005), pp. 427-444.

[CPZ05] M. Carreira-Perpinán and R. Zemel. Proximity graphs for clustering and manifold learning. Advances in Neural Information Processing Systems 17 (2005), pp. 225-232.

[Cay05] L. Cayton. Algorithms for manifold learning. Tech. rep. Department of Computer Science, University of California at San Diego, 2005.

[CD06] L. Cayton and S. Dasgupta. Robust Euclidean embedding. In Proceedings of the 23rd International Conference on Machine Learning. 2006, pp. 169-176.

$\left[\mathrm{Che}^{+} 20\right]$ S. Chen, S. Ma, A. Man-Cho So, and T. Zhang. Proximal gradient method for nonsmooth optimization over the Stiefel manifold. SIAM Journal on Optimization 30.1 (2020), pp. 210-239.

[CWC13] W. Chen, K. Weinberger, and Y. Chen. Maximum variance correction with application to $\mathrm{A}^{*}$ search. In International Conference on Machine Learning. 2013, pp. 302-310.

[Che $\left.{ }^{+} 17\right]$ Y. Chen, C. Ding, J. Hu, R. Chen, P. Hui, and X. Fu. Building and analyzing a global co-authorship network using Google Scholar Data. In Proceedings of the 26th International Conference on World Wide Web Companion. 2017, pp. 1219-1224.

[CG97] F. Chung and F. Graham. Spectral Graph Theory. 92. American Mathematical Society, 1997. 
[CDG18] S. Corbett-Davies and S. Goel. The measure and mismeasure of fairness: A critical review of fair machine learning. arXiv (2018).

[CC00] T. Cox and M. Cox. Multidimensional Scaling. CRC Press, 2000.

[Dev20] J. Devlin. BERT. 2020. URL: https://github.com/google-research/ bert.

[Dev ${ }^{+}$19] J. Devlin, M.-W. Chang, K. Lee, and K. Toutanova. BERT: Pre-training of Deep Bidirectional Transformers for Language Understanding. In Proceedings of the 2019 Conference of the North American Chapter of the Association for Computational Linguistics: Human Language Technologies. 2019, pp. 4171-4186.

$\left[\mathrm{Dia}^{+} 17\right] \quad$ I. Diakonikolas, G. Kamath, D. Kane, J. Li, A. Moitra, and A. Stewart. Being robust (in high dimensions) can be practical. In International Conference on Machine Learning. 2017, pp. 999-1008.

[Dok $\left.{ }^{+} 15\right] \quad$ I. Dokmanic, R. Parhizkar, J. Ranieri, and M. Vetterli. Euclidean distance matrices: Essential theory, algorithms, and applications. IEEE Signal Processing Magazine 32.6 (2015), pp. 12-30.

[DCL11] W. Dong, M. Charikar, and K. Li. Efficient $k$-nearest neighbor graph construction for generic similarity measures. In Proceedings of the 20th International Conference on World Wide Web. 2011, pp. 577-586.

$\left[\right.$ Dwo $\left.^{+} 12\right] \quad$ C. Dwork, M. Hardt, T. Pitassi, O. Reingold, and R. Zemel. Fairness through awareness. In Proceedings of the 3rd Innovations in Theoretical Computer Science Conference. 2012, pp. 214-226.

$\left[\mathrm{Dwo}^{+} 01\right]$ C. Dwork, R. Kumar, M. Naor, and D. Sivakumar. Rank aggregation methods for the web. In Proceedings of the 10th International Conference on World Wide Web. 2001, pp. 613-622.

[Ead84] P. Eades. A heuristic for graph drawing. In Proceedings of the 13th Manitoba Conference on Numerical Mathematics and Computing. Vol. 42. 1984, pp. 149-160.

[EK10] D. Easley and J. Kleinberg. Networks, Crowds, and Markets. Vol. 8. Cambridge University Press, 2010.

[EY36] C. Eckart and G. Young. The approximation of one matrix by another of lower rank. Psychometrika 1.3 (1936), pp. 211-218.

[EAS98] A. Edelman, T. Arias, and S. Smith. The geometry of algorithms with orthogonality constraints. SIAM Journal on Matrix Analysis and Applications 20.2 (1998), pp. 303-353. 
$\left[\mathrm{EA}^{+} 16\right] \quad$ A. El Alaoui, X. Cheng, A. Ramdas, M. Wainwright, and M. Jordan. Asymptotic behavior of $\ell_{p}$-based Laplacian regularization in semi-supervised learning. In Conference on Learning Theory. 2016, pp. 879-906.

$\left[\operatorname{Eps}^{+} 12\right] \quad$ S. Epskamp, A. Cramer, L. Waldorp, V. Schmittmann, and D. Borsboom. qgraph: Network visualizations of relationships in psychometric data. Journal of Statistical Software 48.4 (2012), pp. 1-18.

[FH55] K. Fan and A. Hoffman. Some metric inequalities in the space of matrices. Proceedings of the American Mathematical Society 6.1 (1955), pp. 111-116.

[FCW67] C. Fisk, d. Caskey, and L. West. ACCEL: Automated circuit card etching layout. Proceedings of the IEEE 55.11 (1967), pp. 1971-1982.

[Fle70] R. Fletcher. A new approach to variable metric algorithms. The Computer Journal 13.3 (1970), pp. 317-322.

[FV86] M. Fligner and J. Verducci. Distance based ranking models. Journal of the Royal Statistical Society: Series B (Methodological) 48.3 (1986), pp. 359-369.

[GN00] E. Gansner and S. North. An open graph visualization system and its applications to software engineering. Software - Practice and Experience 30.11 (2000), pp. 1203-1233.

[Gar $\left.{ }^{+} 18\right] \quad$ N. Garg, L. Schiebinger, D. Jurafsky, and J. Zou. Word embeddings quantify 100 years of gender and ethnic stereotypes. Proceedings of the National Academy of Sciences 115.16 (2018), E3635-E3644.

[GMS02] P. Gill, W. Murray, and M. Saunders. SNOPT: an SQP algorithm for large-scale constrained optimization. SIAM Journal on Optimization 12.4 (2002), pp. 979-1006.

[Gol70] D. Goldfarb. A family of variable-metric methods derived by variational means. Mathematics of Computation 24.109 (1970), pp. 23-26.

[GVL13] G. Golub and C. Van Loan. Matrix Computations. Fourth. Johns Hopkins Studies in the Mathematical Sciences. Johns Hopkins University Press, Baltimore, MD, 2013.

[GBC16] I. Goodfellow, Y. Bengio, and A. Courville. Deep Learning. MIT Press, 2016.

[Goo] Google. Google Scholar. https://scholar.google.com/. Accessed: November 2020. 
[GR87] L. Greengard and V. Rokhlin. A fast algorithm for particle simulations. Journal of Computational Physics 73.2 (1987), pp. 325-348.

[GLM96] P. Groenen, J. de Leeuw, and R. Mathar. Least squares multidimensional scaling with transformed distances. In From Data to Knowledge. Springer, 1996, pp. 177-185.

[GL16] A. Grover and J. Leskovec. node2vec: Scalable feature learning for networks. In Proceedings of the 22nd ACM SIGKDD International Conference on Knowledge Discovery and Data Mining. 2016, pp. 855-864.

[HSS08] A. Hagberg, D. Schult, and P. Swart. Exploring network structure, dynamics, and cunction using NetworkX. In Proceedings of the 7th Python in Science Conference. 2008, pp. $11-15$.

[Hal70] K. Hall. An $r$-dimensional quadratic placement algorithm. Management Science 17.3 (1970), pp. 219-229.

$\left[\mathrm{Ham}^{+}\right.$04] J. Ham, D. Lee, S. Mika, and B. Schölkopf. A kernel view of the dimensionality reduction of manifolds. In International Conference on Machine Learning. 2004, p. 47.

[HYL17] W. Hamilton, R. Ying, and J. Leskovec. Representation learning on graphs: Methods and applications. arXiv (2017).

$\left[\mathrm{Hay}^{+}\right.$91] T. Hayden, J. Wells, W.-M. Liu, and P. Tarazaga. The cone of distance matrices. Linear Algebra and its Applications 144 (1991), pp. 153-169.

$\left[\mathrm{He}^{+} 16\right] \quad$ K. He, X. Zhang, S. Ren, and J. Sun. Deep residual learning for image recognition. In Proceedings of the IEEE Conference on Computer Vision and Pattern Recognition. 2016, pp. 770-778.

[Hig89] N. Higham. Matrix nearness problems and applications. In Applications of Matrix Theory. Vol. 22. Oxford University Press, New York, 1989, pp. $1-27$.

[HR03] G. Hinton and S. Roweis. Stochastic neighbor embedding. In Advances in Neural Information Processing Systems. 2003, pp. 857-864.

[HUL93] J.-B. Hiriart-Urruty and C. Lemaréchal. Convex Analysis and Minimization Algorithms I. Fundamentals. Vol. 305. Grundlehren der Mathematischen Wissenschaften [Fundamental Principles of Mathematical Sciences]. Springer-Verlag, Berlin, 1993.

[Hir05] J. Hirsch. An index to quantify an individual's scientific research output. Proceedings of the National Academy of Sciences 102.46 (2005), pp. 16569-16572. 
$\left[\mathrm{Hol}^{+}{ }^{19]}\right.$ K. Holstein, J. Wortman Vaughan, H. Daumé III, M. Dudik, and H. Wallach. Improving fairness in machine learning systems: What do industry practitioners need? In Proceedings of the 2019 CHI Conference on Human Factors in Computing Systems. 2019, pp. 1-16.

[HHY18] S. Hosseini, W. Huang, and R. Yousefpour. Line search algorithms for locally Lipschitz functions on Riemannian manifolds. SIAM Journal on Optimization 28.1 (2018), pp. 596-619.

[Hot33] H. Hotelling. Analysis of a complex of statistical variables into principal components. Journal of Educational Psychology 24.6 (1933), p. 417.

$\left[\mathrm{Hu}^{+} 19\right] \quad J . \mathrm{Hu}, \mathrm{B}$. Jiang, L. Lin, Z. Wen, and Y. Yuan. Structured quasi-Newton methods for optimization with orthogonality constraints. SIAM Journal on Scientific Computing 41.4 (2019), A2239-A2269.

[HAG18] W. Huang, P.-A. Absil, and K. Gallivan. A Riemannian BFGS method without differentiated retraction for nonconvex optimization problems. SIAM Journal on Optimization 28.1 (2018), pp. 470-495.

[HAG17] W. Huang, P.-A. Absil, and K. Gallivan. Intrinsic representation of tangent vectors and vector transports on matrix manifolds. Numerische Mathematik 136.2 (2017), pp. 523-543.

[HGA15] W. Huang, K. Gallivan, and P.-A. Absil. A Broyden class of quasiNewton methods for Riemannian optimization. SIAM Journal on Optimization 25.3 (2015), pp. 1660-1685.

[Hug20] HuggingFace. Transformers. 2020. URL: https://github.com/huggingface/ transformers.

$\left[\mathrm{Hut}^{+} 20\right] \quad$ B. Hutchinson, V. Prabhakaran, E. Denton, K. Webster, Y. Zhong, and S. Denuyl. Social Biases in NLP Models as Barriers for Persons with Disabilities. In Proceedings of the 58th Annual Meeting of the Association for Computational Linguistics. 2020, pp. 5491-5501.

[Hut89] M. Hutchinson. A stochastic estimator of the trace of the influence matrix for Laplacian smoothing splines. Communications in Statistics Simulation and Computation 18.3 (1989), pp. 1059-1076.

[IMS17] P. Indyk, J. Matoušek, and A. Sidiropoulos. Low-distortion embeddings of finite metric spaces. In Handbook of Discrete and Computational Geometry. Ed. by C. D. Toth, J. O'Rourke, and J. E. Goodman. Chapman and Hall/CRC, 2017. Chap. 8, pp. 211-231. 
[JD17] T. Jensen and M. Diehl. An approach for analyzing the global rate of convergence of quasi-Newton and truncated-Newton methods. Journal of Optimization Theory and Applications 172.1 (2017), pp. 206-221.

[Ji07] H. Ji. Optimization approaches on smooth manifolds. PhD thesis. Australian National University, 2007.

[JD15] B. Jiang and Y.-H. Dai. A framework of constraint preserving update schemes for optimization on Stiefel manifold. Mathematical Programming 153.2 (2015), pp. 535-575.

[Joa02] T. Joachims. Optimizing search engines using clickthrough data. In Proceedings of the 8th ACM SIGKDD International Conference on Knowledge Discovery and Data Mining. 2002, pp. 133-142.

[JL84] W. Johnson and J. Lindenstrauss. Extensions of Lipschitz mappings into a Hilbert space. Contemporary Mathematics 26.189-206 (1984), p. 1.

[KK89] T. Kamada and S. Kawai. An algorithm for drawing general undirected graphs. Information Processing Letters 31.1 (1989), pp. 7-15.

[Kny18] A. Knyazev. On spectral partitioning of signed graphs. In 2018 Proceedings of the Seventh SIAM Workshop on Combinatorial Scientific Computing. SIAM. 2018, pp. 11-22.

[Kny17] A. Knyazev. Signed Laplacian for spectral clustering revisited. arXiv (2017).

[Kny01] A. Knyazev. Toward the optimal preconditioned eigensolver: Locally optimal block preconditioned conjugate gradient method. SIAM Journal on Scientific Computing 23.2 (2001), pp. 517-541.

[KB19] D. Kobak and P. Berens. The art of using t-SNE for single-cell transcriptomics. Nature Communications 10.1 (2019), pp. 1-14.

[Kob12] S. Kobourov. Spring embedders and force directed graph drawing algorithms. arXiv (2012).

[KKK20] M. Kochurov, R. Karimov, and S. Kozlukov. Geoopt: Riemannian optimization in PyTorch. arXiv (2020).

[KCS11] E. Kokiopoulou, J. Chen, and Y. Saad. Trace optimization and eigenproblems in dimension reduction methods. Numerical Linear Algebra with Applications 18.3 (2011), pp. 565-602.

[Kor03] Y. Koren. On spectral graph drawing. In International Computing and Combinatorics Conference. Springer. 2003, pp. 496-508. 
[Kru64a] J. Kruskal. Multidimensional scaling by optimizing goodness of fit to a nonmetric hypothesis. Psychometrika 29.1 (1964), pp. 1-27.

[Kru64b] J. Kruskal. Nonmetric multidimensional scaling: A numerical method. Psychometrika 29.2 (1964), pp. 115-129.

$\left[\mathrm{Kun}^{+}{ }^{10}\right]$ J. Kunegis, S. Schmidt, A. Lommatzsch, J. Lerner, E. De Luca, and S. Albayrak. Spectral analysis of signed graphs for clustering, prediction and visualization. In Proceedings of the 2010 SIAM International Conference on Data Mining. SIAM. 2010, pp. 559-570.

[Lan51] C. Lanczos. An iteration method for the solution of the eigenvalue problem of linear differential and integral operators. In Proceedings of a Second Symposium on Large-Scale Digital Calculating Machinery. Harvard University Press, 1951, pp. 164-206.

[Law11] N. Lawrence. Spectral dimensionality reduction via maximum entropy. In International Conference on Artificial Intelligence and Statistics. 2011, pp. 51-59.

[LM14] Q. Le and T. Mikolov. Distributed representations of sentences and documents. In International Conference on Machine Learning. 2014, pp. 1188-1196.

[LCB98] Y. LeCun, C. Cortes, and C. Burges. The MNIST database of handwritten digits. 1998. URL: http://yann. lecun.com/exdb/mnist/.

[LS99] D. Lee and S. Seung. Learning the parts of objects by non-negative matrix factorization. Nature 401.6755 (1999), pp. 788-791.

$\left[\mathrm{Lib}^{+} 14\right] \quad$ L. Liberti, C. Lavor, N. Maculan, and A. Mucherino. Euclidean distance geometry and applications. SIAM Review 56.1 (2014), pp. 3-69.

[LZ08] T. Lin and H. Zha. Riemannian manifold learning. IEEE Transactions on Pattern Analysis and Machine Intelligence 30.5 (2008), pp. 796-809.

[LLR95] N. Linial, E. London, and Y. Rabinovich. The geometry of graphs and some of its algorithmic applications. Combinatorica 15.2 (1995), pp. 215245.

[Luc12] R. Luce. Individual choice behavior: A theoretical analysis. Courier Corporation, 2012.

[MF11] Y. Ma and Y. Fu. Manifold Learning Theory and Applications. CRC press, 2011. 
[MH08] L. van der Maaten and G. Hinton. Visualizing data using t-SNE. Journal of Machine Learning Research 9 (2008), pp. 2579-2605.

[Man02] J. Manton. Optimization algorithms exploiting unitary constraints. IEEE Transactions on Signal Processing 50.3 (2002), pp. 635-650.

[Mar70] B. Martinet. Brève communication. Régularisation d'inéquations variationnelles par approximations successives. Revue française d'informatique et de recherche opérationnelle. Série rouge 4.R3 (1970), pp. 154-158.

[McI20a] L. McInnes. pynndescent. 2020. URL: https://github.com/Imcinnes/ pynndescent.

[McI20b] L. McInnes. UMAP. 2020. URL: https://github.com/Imcinnes/umap.

[MHM18] L. McInnes, J. Healy, and J. Melville. UMAP: Uniform manifold approximation and projection for dimension reduction. arXiv (2018).

[Meg $\left.{ }^{+} 18\right]$ M. Meghwanshi, P. Jawanpuria, A. Kunchukuttan, H. Kasai, and B. Mishra. McTorch, a manifold optimization library for deep learning. $\operatorname{arXiv}(2018)$.

[Men28] K. Menger. Untersuchungen über allgemeine Metrik. Mathematische Annalen 100.1 (1928), pp. 75-163.

$\left[\mathrm{Mey}^{+} 20\right]$ R. Meyer, C. Musco, C. Musco, and D. Woodruff. Hutch++: Optimal stochastic trace estimation. arXiv (2020).

$\left[\mathrm{Mik}^{+} 13\right]$ T. Mikolov, I. Sutskever, K. Chen, G. Corrado, and J. Dean. Distributed representations of words and phrases and their compositionality. In $A d$ vances in Neural Information Processing Systems. 2013, pp. 3111-3119.

[Nar $\left.{ }^{+} 17\right]$ A. Narayanan, M. Chandramohan, L. Rajasekar Venkatesan, Y.-L. Chen, and S. Jaiswal. graph2vec: Learning distributed representations of graphs. In Workshop on Mining and Learning with Graphs. 2017.

$\left[\mathrm{Nel}^{+}\right.$08] M. Nelson, K. Bryc, K. King, A. Indap, A. Boyko, J. Novembre, L. Briley, Y. Maruyama, D. Waterworth, G. Waeber, et al. The Population Reference Sample, POPRES: a resource for population, disease, and pharmacological genetics research. The American Journal of Human Genetics 83.3 (2008), pp. 347-358.

[Net] NetworkLayout.jl. 2020. URL: https : / / github . com / JuliaGraphs / NetworkLayout.jl.

[Ng17] P. Ng. dna2vec: Consistent vector representations of variable-length kmers. arXiv (2017). 
[NK17] M. Nickel and D. Kiela. Poincaré embeddings for learning hierarchical representations. Advances in Neural Information Processing Systems 30 (2017), pp. 6338-6347.

[Noc80] J. Nocedal. Updating quasi-Newton matrices with limited storage. Mathematics of Computation 35.151 (1980), pp. 773-782.

[NW06] J. Nocedal and S. Wright. Numerical Optimization. Second. Springer Series in Operations Research and Financial Engineering. Springer, New York, 2006.

$\left[\mathrm{Nov}^{+} 08\right] \quad$ J. Novembre, T. Johnson, K. Bryc, Z. Kutalik, A. Boyko, A. Auton, A. Indap, K. King, S. Bergmann, M. Nelson, et al. Genes mirror geography within Europe. Nature 456.7218 (2008), pp. 98-101.

[Pag $\left.{ }^{+} 99\right] \quad$ L. Page, S. Brin, R. Motwani, and T. Winograd. The PageRank citation ranking: Bringing order to the web. Tech. rep. Stanford InfoLab, 1999.

[PB14] N. Parikh and S. Boyd. Proximal algorithms. Foundations and Trends in Optimization 1.3 (2014), pp. 127-239.

$\left[\mathrm{Pas}^{+} 19\right] \quad$ A. Paszke, S. Gross, F. Massa, A. Lerer, J. Bradbury, G. Chanan, T. Killeen, Z. Lin, N. Gimelshein, L. Antiga, et al. PyTorch: An imperative style, high-performance deep learning library. In Advances in Neural Information Processing Systems. 2019, pp. 8024-8035.

[Pea01] K. Pearson. On lines and planes of closest fit to systems of points in space. The London, Edinburgh, and Dublin Philosophical Magazine and Journal of Science 2.11 (1901), pp. 559-572.

$\left[\right.$ Ped $\left.^{+} 11\right] \quad$ F. Pedregosa, G. Varoquaux, A. Gramfort, V. Michel, B. Thirion, O. Grisel, M. Blondel, P. Prettenhofer, R. Weiss, V. Dubourg, et al. Scikitlearn: Machine learning in Python. Journal of Machine Learning Research 12 (2011), pp. 2825-2830.

[PARS14] B. Perozzi, R. Al-Rfou, and S. Skiena. DeepWalk: Online learning of social representations. In Proceedings of the 20th ACM SIGKDD International Conference on Knowledge Discovery and Data Mining. 2014, pp. 701-710.

[Pla75] R. Plackett. The analysis of permutations. Journal of the Royal Statistical Society: Series C (Applied Statistics) 24.2 (1975), pp. 193-202.

[PSZ19] P. Poličar, M. Stražar, and B. Zupan. openTSNE: A modular Python library for t-SNE dimensionality reduction and embedding. bioRxiv (2019). 
[PSL90] A. Pothen, H. Simon, and K.-P. Liou. Partitioning sparse matrices with eigenvectors of graphs. SIAM Journal on Matrix Analysis and Applications 11.3 (1990), pp. 430-452.

[QB79] N. Quinn and M. Breuer. A forced directed component placement procedure for printed circuit boards. IEEE Transactions on Circuits and systems 26.6 (1979), pp. 377-388.

[̌̌S10] R. Řehůřek and P. Sojka. Software Framework for Topic Modelling with Large Corpora. In Proceedings of the LREC 2010 Workshop on New Challenges for NLP Frameworks. ELRA, 2010, pp. 45-50.

[Ric38] M. Richardson. Multidimensional psychophysics. Psychological Bulletin 35 (1938), pp. 659-660.

[RW12] W. Ring and B. Wirth. Optimization methods on Riemannian manifolds and their application to shape space. SIAM Journal on Optimization 22.2 (2012), pp. 596-627.

[Roc76] R Rockafellar. Monotone operators and the proximal point algorithm. SIAM Journal on Control and Optimization 14.5 (1976), pp. 877-898.

[RS00] S. Roweis and L. Saul. Nonlinear dimensionality reduction by locally linear embedding. Science 290.5500 (2000), pp. 2323-2326.

[RB14] E. Ryu and S. Boyd. Stochastic proximal iteration: A non-asymptotic improvement upon stochastic gradient descent (2014).

[Sal $\left.{ }^{+} 18\right] \quad$ F. Sala, C. De Sa, A. Gu, and C. Ré. Representation tradeoffs for hyperbolic embeddings. In International Conference on Machine Learning. 2018, pp. 4460-4469.

[Sam69] J. Sammon. A nonlinear mapping for data structure analysis. IEEE Transactions on Computers 100.5 (1969), pp. 401-409.

[San14] R. Sandberg. Entering the era of single-cell transcriptomics in biology and medicine. Nature Methods 11.1 (2014), pp. 22-24.

[Sau20] L. Saul. A tractable latent variable model for nonlinear dimensionality reduction. Proceedings of the National Academy of Sciences 117.27 (2020), pp. 15403-15408.

[SR01] L. Saul and S. Roweis. An introduction to locally linear embedding. Tech. rep. 2001.

[Sch66] P. Schönemann. A generalized solution of the orthogonal Procrustes problem. Psychometrika 31.1 (1966), pp. 1-10. 
[SCL13] B. Schouten, M. Calinescu, and A. Luiten. Optimizing quality of response through adaptive survey designs. Survey Methodology 39.1 (2013), pp. 29-58.

[Sha70] D. Shanno. Conditioning of quasi-Newton methods for function minimization. Mathematics of Computation 24.111 (1970), pp. 647-656.

[She12] N. Sherwani. Algorithms for VLSI Physical Design Automation. Springer Science \& Business Media, 2012.

[SDJ91] G. Sigl, K. Doll, and F. Johannes. Analytical placement: A linear or a quadratic objective function? In Proceedings of the 28th ACM/IEEE design automation conference. 1991, pp. 427-432.

$\left[\mathrm{Szu}^{+} 19\right] \quad$ B. Szubert, J. Cole, C. Monaco, and I. Drozdov. Structure-preserving visualisation of high dimensional single-cell datasets. Scientific Reports 9.1 (2019), pp. 1-10.

$\left[\mathrm{Tan}^{+}\right.$16] J. Tang, J. Liu, M. Zhang, and Q. Mei. Visualizing large-scale and highdimensional data. In Proceedings of the 25th International Conference on World Wide Web. 2016, pp. 287-297.

$\left[\mathrm{Tan}^{+} 15\right] \quad$ J. Tang, M. Qu, M. Wang, M. Zhang, J. Yan, and Q. Mei. LINE: Largescale information network embedding. In Proceedings of the 24th International Conference on World Wide Web. 2015, pp. 1067-1077.

[TDSL00] J. Tenenbaum, V. De Silva, and J. Langford. A global geometric framework for nonlinear dimensionality reduction. Science 290.5500 (2000), pp. 2319-2323.

[Tor52] W. Torgerson. Multidimensional scaling: I. Theory and method. Psychometrika 17.4 (1952), pp. 401-419.

[TKW16] J. Townsend, N. Koep, and S. Weichwald. PyManopt: A python toolbox for optimization on manifolds using automatic differentiation. The Journal of Machine Learning Research 17.1 (2016), pp. 4755-4759.

[TB97] L. Trefethen and D. Bau. Numerical Linear Algebra. Society for Industrial and Applied Mathematics (SIAM), Philadelphia, PA, 1997.

[Tut63] W. T. Tutte. How to draw a graph. Proceedings of the London Mathematical Society 3.1 (1963), pp. 743-767.

[Ude ${ }^{+}$16] M. Udell, C. Horn, R. Zadeh, S. Boyd, et al. Generalized low rank models. Foundations and Trends in Machine Learning 9.1 (2016), pp. 1-118. 
[Uni] United States Census Bureau. American Community Survey 2013-2017 5-Year Data. https://www. census.gov/newsroom/press-kits/2018/ acs-5year.html. Accessed: November 2020.

[VAD08] L. Von Ahn and L. Dabbish. Designing games with a purpose. Communications of the ACM 51.8 (2008), pp. 58-67.

[vL07] U. von Luxburg. A tutorial on spectral clustering. Statistics and Computing 17.4 (2007), pp. 395-416.

[WB06] A. Wächter and L. Biegler. On the implementation of an interior-point filter line-search algorithm for large-scale nonlinear programming. Mathematical Programming 106.1, Series A (2006), pp. 25-57.

[Wan $\left.{ }^{+} 20\right]$ Y. Wang, H. Huang, C. Rudin, and Y. Shaposhnik. Understanding how dimension deduction tools work: An empirical approach to deciphering t-SNE, UMAP, TriMAP, and PaCMAP for data visualization. arXiv (2020).

[WS04] K. Weinberger and L. Saul. Unsupervised learning of image manifolds by semidefinite programming. In Proceedings of the 2004 IEEE Computer Society Conference on Computer Vision and Pattern Recognition. Vol. 2. 2004.

[WE19] L. White and D. Ellison. Embeddings.jl: Easy access to pretrained word embeddings from Julia. Journal of Open Source Software 4.36 (2019), p. 1013.

[Wil $\left.{ }^{+} 20\right] \quad$ A. Wilk, A. Rustagi, N. Zhao, J. Roque, G. Martínez-Colón, J. McKechnie, G. Ivison, T. Ranganath, R. Vergara, T. Hollis, et al. A single-cell atlas of the peripheral immune response in patients with severe COVID19. Nature Medicine (2020), pp. 1-7.

$\left[\right.$ Wil $\left.^{+} 14\right] \quad$ R. Wilson, E. Hancock, E. Pekalska, and R. Duin. Spherical and hyperbolic embeddings of data. IEEE Transactions on Pattern Analysis and Machine Intelligence 36.11 (2014), pp. 2255-2269.

[Xu10] Y. Xu. Semi-supervised Learning on Graphs: A Statistical Approach. PhD thesis. Stanford University, 2010.

[Yan $\left.{ }^{+} 06\right] \quad$ S. Yan, D. Xu, B. Zhang, H.-J. Zhang, Q. Yang, and S. Lin. Graph embedding and extensions: A general framework for dimensionality reduction. IEEE Transactions on Pattern Analysis and Machine Intelligence 29.1 (2006), pp. 40-51. 
[YH38] G. Young and A. Householder. Discussion of a set of points in terms of their mutual distances. Psychometrika 3.1 (1938), pp. 19-22.

[ZXQ19] S. Zhou, N. Xiu, and H.-D. Qi. Robust Euclidean embedding via EDM optimization. Mathematical Programming Computation (2019), pp. 151 .

$\left[\mathrm{Zhu}^{+}{ }^{19}\right]$ Z. Zhu, S. Xu, M. Qu, and J. Tang. GraphVite: A high-performance CPU-GPU hybrid system for node embedding. In Proceedings of the World Wide Web Conference. 2019, pp. 2494-2504. 